University of Louisville

ThinkIR: The University of Louisville's Institutional Repository

$8-2020$

\title{
Comparative secretomics and functional analysis of effectors utilized by the Microbotryum genus of anther-smut fungal pathogens, and their role in host-specificity.
}

William Christopher Beckerson

University of Louisville

Follow this and additional works at: https://ir.library.louisville.edu/etd

Part of the Genomics Commons, and the Molecular Genetics Commons

\section{Recommended Citation}

Beckerson, William Christopher, "Comparative secretomics and functional analysis of effectors utilized by the Microbotryum genus of anther-smut fungal pathogens, and their role in host-specificity." (2020). Electronic Theses and Dissertations. Paper 3496.

https://doi.org/10.18297/etd/3496

This Doctoral Dissertation is brought to you for free and open access by ThinkIR: The University of Louisville's Institutional Repository. It has been accepted for inclusion in Electronic Theses and Dissertations by an authorized administrator of ThinkIR: The University of Louisville's Institutional Repository. This title appears here courtesy of the author, who has retained all other copyrights. For more information, please contact thinkir@louisville.edu. 


\title{
COMPARATIVE SECRETOMICS AND FUNCTIONAL ANALYSIS OF EFFECTORS UTILIZED BY THE MICROBOTRYUM GENUS OF ANTHER-SMUT FUNGAL PATHOGENS, AND THEIR ROLE IN HOST-SPECIFICITY
}

\author{
By \\ William Christopher Beckerson, M.Sc.
}

\author{
A Dissertation \\ Submitted to the Faculty of the University of Louisville in Partial Fulfillment of the Requirements \\ for the Degree of: \\ Doctor of Philosophy in Biology \\ Department of Biology: Program on Disease Evolution, \\ Molecular, Cellular, and Developmental Biology Division, \\ College of Arts and Sciences \\ University of Louisville \\ Louisville, Kentucky, United States
}

August 2020 
Copyright 2020 by William Christopher Beckerson

(C) All rights reserved 



\section{COMPARATIVE SECRETOMICS AND FUNCTIONAL ANALYSIS OF EFFECTORS}

UTILIZED BY THE MICROBOTRYUM GENUS OF ANTHER-SMUT FUNGAL

PATHOGENS, AND THEIR ROLE IN HOST-SPECIFICITY

By

William Christopher Beckerson, M.Sc.

Dissertation Approved on:

July $17^{\text {th }}, 2020$

By the following members of the Dissertation Committee:

Dr. Michael H. Perlin, Principal Advisor

Dr. David Schultz

Dr. Susanna Remold

Dr. Lee Dugatkin

Dr. Scott Gold 


\section{ACKNOWLEDMENTS}

\section{"If I have seen further than others, it is by standing on the shoulders of giants" Isaac Newton}

The success I have experienced throughout my research at the University of Louisville is due in great part to the many collaborations I have been a part of, both at the University of Louisville and Universities across the world. I would like to express my deepest gratitude towards the following groups for their guidance and openness towards research, practices that embody the essence of science and have allowed us to accomplish more together than I ever could have on my own.

\section{The Giraud Lab at the Université Paris-Sud:}

I would first like to thank Dr. Tatiana Giraud for accepting me into her lab early on in my dissertation and for her guidance across a wide variety of research projects during the span of my dissertation. Dr. Giraud is one of the most effective researchers I have ever had the privilege of working with and has been an excellent role model for me. I would also like to thank Dr. Ricardo de la Vega for his invaluable bioinformatic contributions, as well as Dr. Fanny Hartmann and Marine Duhamel for their contributions to our collaborative secretome research.

\section{The Begerow Lab at Ruhr Universität Bochum:}

Dr. Dominik Begerow is another individual I would like to think for his incredibly gracious hospitality throughout my research with his lab in Germany, and for his guidance regarding collaborative efforts between the Perlin and Begerow labs. I would also like to extend a special thank you to Sebastian Klenner, one of the first individuals I had the pleasure of working with during my dissertation, a great scientist, and a great friend. I would also like to thank Lucas Engelhardt, another excellent researcher from the Begerow lab, as well as Martin Kemler; an astute taxonomist, avid bird watcher, and a wonderful drinking buddy!

\section{The Gold Lab at the USDA:}

I would like to thank Dr. Scott Gold for his generous contributions to our research, particularly for his help in establishing the first reliable transformation system in Microbotryum and for his contributions to our 
CRISPR research project. Dr. Gold's guidance and insight into fungal transformation has been an essential for establishing transformation methods in Microbotryum.

\section{Dr. Yoder-Himes at the University of Louisville:}

While this dissertation presents my research into the evolution of disease at the University of Louisville, I have also had the privilege to work with Dr. Yoder-Himes to analyze pedagogical practices in Biology. I would like to thank her for the opportunity to join her research team, including Dr. Jen Anderson and Dr. John Perpich, and for the opportunity to peruse teaching questions in effort to be the best instructor I can be.

\section{The Menze Lab at the University of Louisville:}

I would also like to thank Dr. Michael Menze, another collaborator with whom I worked on a variety of small research projects, as well as Clinton Belot, Rob Skolik, and David Grimm, and the rest of the Menze lab for their comradery and encouragement throughout my dissertation.

The Perlin Lab "aka the GOAT Lab" at the University of Louisville:

I would further like to thank my colleagues in the GOAT lab; Hector Mendoza, Sunita Khanal, Ming-chang "Nelson" Tsai, Joseph Paul Ham, Roxanne Leiter, and Swathi Kuppireddy, all of whom have made working in the GOAT lab a wonderful experience. Their willingness to work as a team, even on projects different from their own, facilitated a cooperative environment that made research fun and effective. I would also like to thank the many undergraduates in the GOAT lab who assisted directly with the completion of this work. Thank you to Catarina Cahill, Brittany Carman, Adney Rakotoniaina, Lloyd Bartley, Grace Long, Phillip Sullivan, and Rebecca Turney for your wiliness to learn and in turn teach me how to be a better mentor.

I would also like to extend a special thank you to the late SuSan Toh, whose work in the Perlin lab laid the foundation for this entire dissertation. While I did not know SuSan personally, her impact on the Microbotryum community through her research on Microbotryum lychnidis-dioiace opened the door for many research opportunities and enabled me to peruse the kinds of research questions that I have. 


\section{Graduate Committee Members:}

I would like to thank my graduate committee; Dr. Michael Perlin, Dr. David Schultz, Dr. Lee Dugatkin, Dr. Susanna Remold, and Dr. Scott Gold. When selecting members for this committee, I chose individuals who are experts in fields that I had the least experience in, and whose passion for science was contagious. Doing so allowed me to surround myself with a team that I could readily seek advice from in areas where I needed it the most. I am therefore deeply appreciative for their patience with my many questions over the years, and for their guidance that has made me a much more well-rounded scientist today.

\section{Faculty and Staff of the University of Louisville:}

In addition to those who contributed directly to my research, I would also like to thank the staff and administration at the University of Louisville, whose hard work and dedication to the department has been paramount to my success. Thank you to Dorris Meadows, Terri Norris, and Charice Johnson for frequent assistance with travel, grants, scheduling for the Biology Graduate Student Association, and a plethora of random questions I've had over the years. I would also like to thank two faculty members in particular for their amazing work as Director of Graduate Studies during my dissertation. Thank you Dr. Sarah Emery for being there for students in need during a particularly difficult period for the UofL Biology Department, and a special thank you for your assistance with navigating university policy so that I could accept a wonderful opportunity to teach as an adjunct faculty at my alma mater, Georgetown College. And thank you Dr. Perri Eason for your frequent help as Director of Graduate Studies in the early part of my dissertation work and for your excellent work as the Chair of the Biology Department, particularly your willingness to assist the Biology Graduate Student Association. I would also like to thank the rest of the UofL faculty who have always been available to talk with regarding research question, even if they had no stake in the mater. Their collective cordial and collaborative nature make the University of Louisville a welcoming environment.

\section{My Wonderful Family:}

No one plans to defend their dissertation during a global pandemic, and it is no overstatement to say that

without the support of my family I would have been unable to do so. Continued encouragement and reassurance from my parents, Darla and Chris Beckerson, were paramount in these uncertain times. Through 
anxiety and hardship, they have always been there for me, along with my three brothers, Jon, Alex, and Nick, and of course Brooklyn and Meagan as well. Thank you all so much for being there for me when I needed it the most.

My loving wife:

And finally, I would like to thank my wife Mary Beckerson for her support through the entirety of this long and challenging process. During my dissertation, I spent a total of 8 months abroad working with collaborators across Europe, opportunities that would not have been possible without the support, emotional and otherwise, of Mary. In addition to supporting me during long periods of travel, her love and understanding towards late nights in the lab and weekend research has allowed me to be both successful in my endeavors and achieve some essence of work-life balance. Without her love and support, none of this would have been possible.

\section{Financial Support:}

This work was supported additionally through grants from the National Institutes of Health (sub-award \#OGMB131493C1] to [Michael Perlin] from [P20GM103436] to [Nigel Cooper), PI]), the National Science Foundation (NSF/IRES Award\#1824851) to Dr. Michael Perlin, The DAAD Short Term Research Grant to William C. Beckerson, The Chateaubriand Fellowship to William C. Beckerson, and through various groups at the University of Louisville, including the Graduate Student Council, the Graduate Network of Arts and Science, and the Biology Graduate Student Association. 


\begin{abstract}
COMPARATIVE SECRETOMICS AND FUNCTIONAL ANALYSIS OF EFFECTORS
\end{abstract}

UTILIZED BY THE MICROBOTRYUM GENUS OF ANTHER-SMUT FUNGAL

PATHOGENS, AND THEIR ROLE IN HOST-SPECIFICITY

William Christopher Beckerson

July $14^{\text {th }}, 2020$

Understanding how pathogens evolve in response to changes in their host is paramount to combating the spread of emergent strains of disease. This is particularly true for plant pathogens that cause billions of dollars of damages to crops globally, every year. Understanding the molecular interactions between pathogens and their hosts therefore sheds light on the coevolutionary arms race that can result in host-specificity and hostshifts in plant pathogens. This research approaches the question of how fungal pathogens interact with their plant hosts utilizing both unique and shared arsenals of secreted proteins (SPs) during infection, and addresses the question of whether alterations to shared SPs or species-specific SPs play a more important role in host-specificity. To answer these questions, we annotated and compared the secretomes of three species from the Microbotryum genus of anther smuts, two closely related sister species that are able to infect each other's hosts, albeit to reduced degrees, M. lychnidis-dioicae and M. silenesdioicae, and one distantly related species that is unable to infect either of the other two species' host plants and vice versa, $M$. violaceum var. paradoxa. We then characterized the function of the core SP MVLG_02245, an SP found in all three species with differing levels of conservation at the amino acid sequence level, and tested the importance of two speciesspecific SPs in host specificity, MvS1_01693 and MvSd_09295, via heterologous expression in each sister species. Finally, for future research into the role of SPs in host pathogenicity, we established a site-specific knockout system in Microbotryum using CRISPR Cas9 technology. Our results demonstrate that while host specificity in the Microbotryum genus is likely the result of alterations to the amino acid sequence of several core SPs, expression of novel SPs can have dramatic effects on pathogenicity. The research is therefore the first to identify key proteins involved in host specificity of the Microbotryum genus, and the first to establish a means of site-specific gene modification and knockout in the Microbotryum system using a CRISPR Cas9. 


\section{TABLE OF CONTENTS}

ACKNOWLEDGMENTS $\ldots \ldots \ldots \ldots \ldots \ldots \ldots \ldots \ldots \ldots \ldots \ldots \ldots$ iii

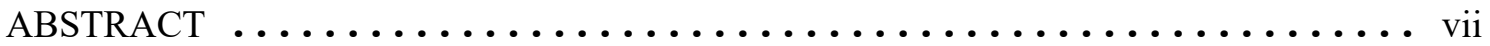

LIST OF TABLES $\ldots \ldots \ldots \ldots \ldots \ldots \ldots \ldots \ldots \ldots \ldots \ldots \ldots \ldots \ldots \ldots \ldots \ldots \ldots$

LIST OF FIGURES $\ldots \ldots \ldots \ldots \ldots \ldots \ldots \ldots \ldots \ldots \ldots \ldots \ldots \ldots \ldots \ldots \ldots \ldots$

CHAPTER 1: An introduction to pathogen/host coevolution and Microbotryum, a model genus

for its study

The symbiotic relationship between plants hosts and their fungal pathogens . . . . . 1

Pathogen/host coevolution and reproductive strategies ............ 2

The role of secretory proteins in pathogen/host coevolution ........... 8

The anther-smut fungi, Microbotryum .................... 13

CHAPTER 2: Identification of conserved and species-specific secreted proteins via comparative secretomics

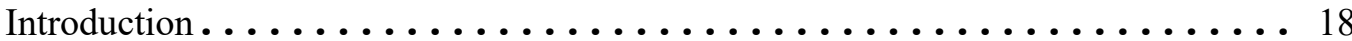

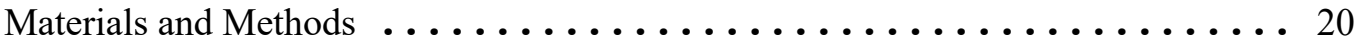

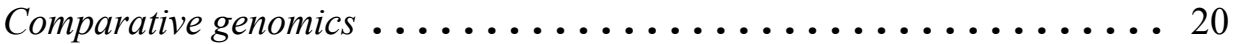

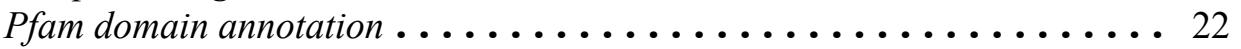

Signal peptide clustering and experimental validation .......... 22

Tests for positive selection .................... 23

Footprints of RIP (repeat-induced point mutations) $\ldots \ldots \ldots \ldots \ldots 25$

Genomic landscape analyses . . . . . . . . . . . . . . 25

Intraspecific secretomes comparisons between M. lychnidis-dioicae isolates

from differentiated populations ................. 26

Analysis of gene expression level across infection stages and mating

conditions ........................ 26

Plotting, statistical tests, and figures ............... 27

Results ................................. 27

Overview of Microbotryum predicted secretomes . . . . . . . . . . . 27

Signal peptide clusters and yeast secretion trap results .......... 32

Interspecies comparison of Microbotryum predicted secretomes . . . . . . 34

Intraspecies comparison of Microbotryum predicted secretomes . . . . . 35

Genomic context of predicted $S P S \ldots \ldots \ldots \ldots \ldots \ldots \ldots \ldots$

Expression of predicted SPs across infection stages ........... 40

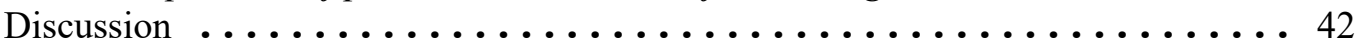

CHAPTER 3: Functional characterization of the conserved small secreted protein MVLG_02245

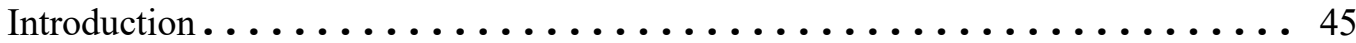

Materials and Methods ......................... 47

Bioinformatic analysis of MVLG_02245 in Microbotryum . . . . . . . . 47

Yeast Secretion Trap of MVLG_02245 . . . . . . . . . . . . . 47

Yeast Two-Hybrid (Y2H) Assay of MVLG_02245 targets . . . . . . . . . 48

Results .............................. 51

Local BLAST Results for Predicted MVLG_02245 Coding Sequence . . . . 51

Predicted Secretion, Function, and Expression of the MVLG_02245 Effector 53

Yeast Secretion Trap and GFP Tagging . . . . . . . . . . . . . 54 
Host Targets of the MVLG_02245 Effector in Silene latifolia . . . . . . . 55

Discussion ............................ 57

CHAPTER 4: Assessing the role of species-specific effectors in host-specialization

Introduction . . .......................... 61

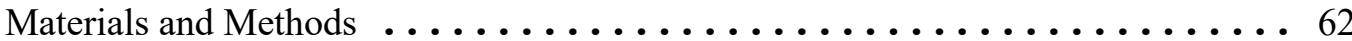

Identifying Species-Specific Small Secreted Proteins . . . . . . . . . 62

EffectorP and Gene Expression ................... 63

ATMT for MvSd 09295 and MvSl 01693 ................ 65

Cross-Infection Analysis for MvSd 09295 in M. lychnidis-dioicae . . . . . 67

Electron Microscopy for MvSd_09295 . . . . . . . . . . . . . . . 71

Cross-Infection Analysis for MvSl_01693 in M. silenes-dioicae . . . . . . 71

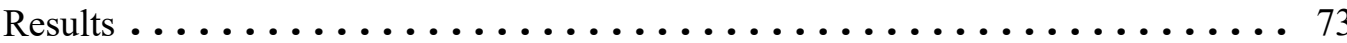

Microscopy Results ..................... 73

Infection results for the MvSd_09295 Ruhr-Universität Bochum study . . . 75

Infection Results for the MvSl_01693 University of Louisville study . . . . . 76

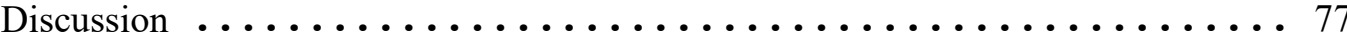

CHAPTER 5: Implementation of CRISPR-Cas9 as an effective target-specific knockout tool for the Microbotryum genus

Introduction.............................. 81

Things to consider before you begin ................ 88

Selecting a transformation method ................ 88

Single plasmids method...................... 88

Agrobacterium-mediated method ................... 90

mRNA-encoded Cas 9 method .................... 91

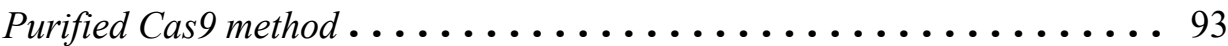

Selecting a suitable Cas 9 variant .................. 95

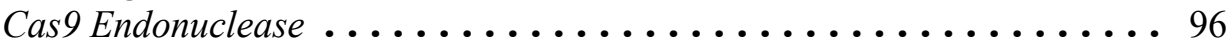

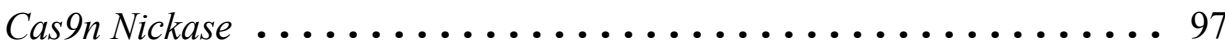

dCas 9 for $C R I S P R i \ldots \ldots \ldots \ldots \ldots \ldots \ldots \ldots \ldots$

High fidelity Cas 9 variants . . . . . . . . . . . . . . . 98

Transposon Associated CRISPR Cas9 .................. 99

Selecting a target ........................... 99

Selecting a promoter for $\operatorname{Cas} 9 \ldots \ldots \ldots \ldots \ldots \ldots \ldots \ldots$

Selecting a promoter for $s g R N A \ldots \ldots \ldots \ldots \ldots \ldots \ldots \ldots \ldots$

Transformation and confirmation of alterations to target genes . . . . . 102

Changing targets and targeting multiple genes . . . . . . . . . 103

Checking for off-target cuts .................... 105

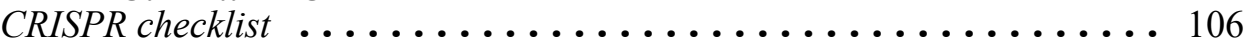

Implementing CRISPR Cas 9 in the Microbotryum genus . . . . . . . 108

Materials and Methods ............................. 109

Selecting a target .......................... 109

Constructing a plasmid for delivery of CRISPR-Cas9 via electroporation • 110

Electroporation of Microbotryum lychnidis-dioicae cells ......... 111

Constructing a plasmid for ATMT delivery of CRISPR-Cas9 . . . . . . 112

Agrobacterium-medicated transformation of $M$. lychnidis-dioicae cells with

pMvHyg CRISPR ....................... 116

Protoplasting Microbotryum cells ................. 117

PEG transformation of Microbotryum with in vitro Cas 9 duplex . . . . . 118

Sequencing to confirm successful knockouts . . . . . . . . . . . . 119 
Results ............................... 120

Electroporation of single plasmid CRISPR Construct . . . . . . . . 120 Agrobacterium-mediated transformation of $M$. lychnidis-dioicae with

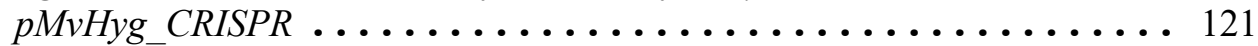
PEG transformation of $M$. lychnidis-dioicae with Cas 9 duplex $\ldots \ldots . . .122$

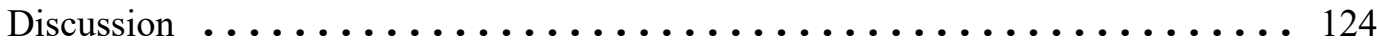

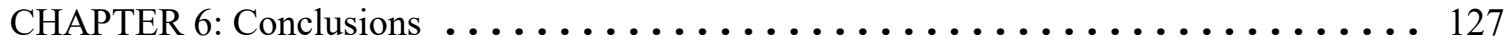

REFERENCES . . . . . . . . . . . . . . . . . . . . . . . . . 129

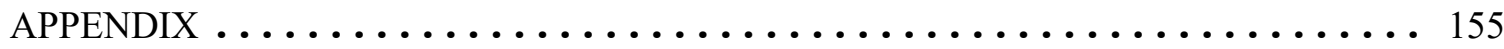

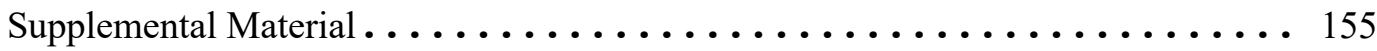

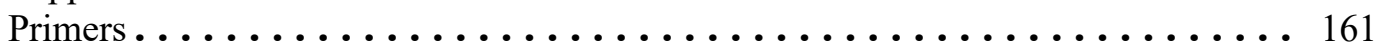

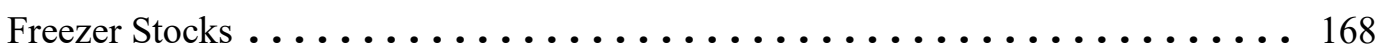

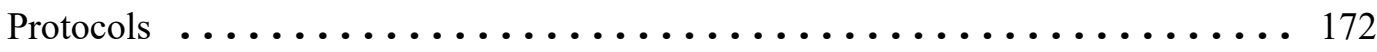

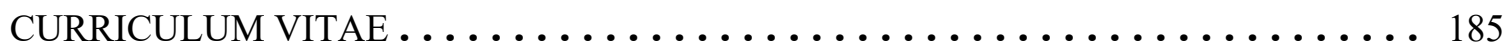




\section{LIST OF TABLES}

Table

Page

Table C1-1 Examples of Microbe-Associated Molecular Patterns . . . . . . . . . . . . . 10

Table C3-1 Expression of MVLG_02245 on various media and in planta, presented as TPM ${ }^{\text {a }} \ldots 52$

Table C4-1 EffectorP 1.0 Results for Species-Specific SPs . . . . . . . . . . . . . . . . . 64

Table C4-2 Expression of MvSl_01693 on various media and in planta, presented as TPM ${ }^{\mathrm{a}}$. . . . 65

Table C4-3 Tally of seedlings surviving, flowering, and infected flowers for the Bochum study $\ldots 75$

Table C4-4 Tally of seedlings surviving, bolting, flowering, and infected flowers for the Louisville

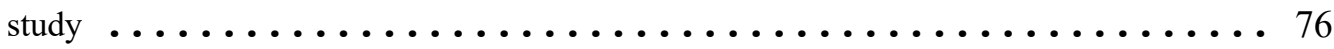

Table C5-1 List of Model Organisms with Established CRISPR Systems . . . . . . . . . 82

Table C5-2 Optimal electroporation settings assay $\ldots \ldots \ldots \ldots \ldots \ldots \ldots \ldots \ldots \ldots$

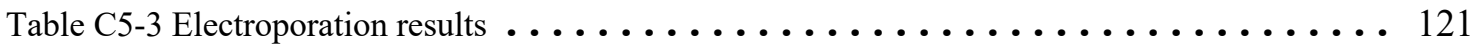




\section{LIST OF FIGURES}

Figure

Page

Figure $\mathrm{C} 1-1$ Genetic variation within a population by reproductive strategies $\ldots \ldots \ldots \ldots \ldots$

Figure C1-2 Elimination of emergent disadvantageous genes through sexually reproduction $\ldots .4$

Figure C1-3 Restoration of wild type phenotype through complementation $\ldots \ldots \ldots \ldots \ldots$

Figure $\mathrm{C} 1-4$ A Model for secreted protein molecular arms race $\ldots \ldots \ldots \ldots \ldots \ldots \ldots \ldots$

Figure C1-5 Comparison of plant and fungal phylogenies using one strain per host species $\ldots \ldots \ldots 13$

Figure C1-6 The life cycle of Microbotryum, demonstrated with M. lychnidis-dioicae $\quad \ldots \ldots \ldots 15$

Figure C2-1 Procedural framework for predicting secreted proteins in three Microbotryum species . 28

Figure C2-2 Comparison between the secretomes from three Microbotryum species . . . . . 30

Figure C2-3 Overview of predicted SP (secreted protein) and non-SP homologs . . . . . . . . 32

Figure $\mathrm{C} 2-4$ Experimental validation of predicted signal peptides $\ldots \ldots \ldots \ldots \ldots \ldots \ldots$

Figure C2-5 Inter- and intra-specific comparisons of Microbotryum secretomes . . . . . . . 37

Figure C2-6 Investigation of the impact of RIP (repeat-induced point mutations) on gene

diversification among species . . . . . . . . . . . . . . . . . . 39

Figure C2-7 Relative expression of Microbotryum lychnidis-dioicae genes across infection stages on

flower structures $\ldots \ldots \ldots \ldots \ldots \ldots \ldots \ldots \ldots \ldots \ldots \ldots \ldots \ldots$

Figure C3-1 The signal peptide region of MVLG_02245 predicted using SignalP4.1 . . . . . . 48

Figure C3-2 Mating verification between Microbotryum cells . . . . . . . . . . . . . . 49

Figure C3-3 Repeat mating verification between Microbotryum cells . . . . . . . . . . 51

Figure C3-4 BlastP comparison between three species of Microbotryum for MVLG_02245 . . . . 52

Figure C3-5 Prediction of disorder in the protein sequence for MVLG_02245 . . . . . . . . . 54

Figure C3-6 Yeast Secretion Trap results for the signal peptide region of MVLG_02245 . . . . . . 55

Figure C3-7 Yeast two-hybrid mating results between MVLG_02245 and t $\alpha-1 \mathrm{c} \ldots \ldots \ldots \ldots$. . . 56

Figure C3-8 SnapGene image of pMvHyg_MVLG_02245-GFP . . . . . . . . . . . . . 59 
Figure C4-1 Computational framework for identification of species-specific SSPs . . . . . . . 63

Figure C4-2 Snap gene image of the vectors for heterologous expression of species-specific genes • 66

Figure C4-3 Confocal image of Agrobacterium-mediated transformation of Microbotryum cells . 67

Figure C4-4 Outline of the planting procedures used in the Ruhr-Universität Bochum study . . . . 69

Figure $\mathrm{C} 4-5$ Greenhouse arrangement of plants in the Bochum study $\ldots \ldots \ldots \ldots \ldots \ldots$. . 70

Figure C4-6Growth chamber conditions for the Louisville study $\ldots \ldots \ldots \ldots \ldots \ldots \ldots$

Figure C4-7 Electron micrographs of seedlings infected with Microbotryum $\ldots \ldots \ldots \ldots \ldots$

Figure $\mathrm{C} 4-8$ Electron micrographs of mating $\mathrm{MvSl}$ a1 and a2 cells $\ldots \ldots \ldots \ldots \ldots \ldots 74$

Figure C4-9 Confirmation of Microbotryum mating via light microscopy . . . . . . . . . 74

Figure C4-10 Infected and non-infected Silene dioicae flowers $\ldots \ldots \ldots \ldots \ldots \ldots \ldots$. . . . 77

Figure C5-1 Diagram of the components required to implement CRISPR Cas9 via a single plasmid

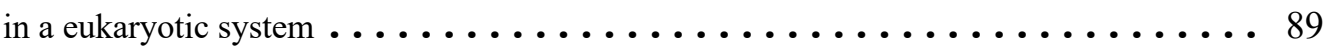

Figure C5-2 Diagram of the components required to implement CRISPR Cas9 via Agrobacterium

mediated transformation in a eukaryotic system $\ldots \ldots \ldots \ldots \ldots \ldots \ldots \ldots$

Figure C5-3 Introduction of CRISPR via mRNA-encoded Cas9 $\ldots \ldots \ldots \ldots \ldots \ldots$

Figure C5-4 Protein purification of Cas9 for introduction to various cell types along with synthetic

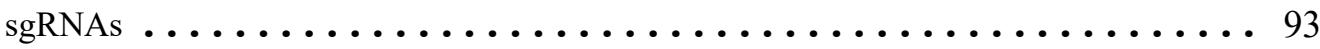

Figure C5-5 Diagram of the differences between sgRNA and crRNA/tracrRNA molecules . . . . 94

Figure C5-6 Illustration of various Cas9 mutants and their amino acid substitutions $\ldots \ldots \ldots 95$

Figure C5-7 Homology directed repair of a Cas9-induced doubled-stranded break in the first exon of a target gene ............................... 97

Figure C5-8 Endogenously driven synthesis of a sgRNA via fusion with a native tRNA . . . . . 102

Figure C5-9 Illustration of easy target replacement in CRISPR Cas9 plasmid constructs . . . . . . 104

Figure C5-10 Checklist, with examples, of components needed to generate a CRISPR Cas9

construct for use in a new system . . . . . . . . . . . . . . . . 107

Figure $\mathrm{C} 5-11$ Plasmid maps for $\mathrm{pMs} 10$ and $\mathrm{pMvCC} 9 \ldots \ldots \ldots \ldots \ldots \ldots \ldots \ldots$ 
Figure C5-12 Stepwise overview of pMvHyg_CRISPR construction via Gibson Overlap PCR . . 113

Figure C5-13 Snapgene image of the pMvHyg_CRISPR plasmid . . . . . . . . . . . 115

Figure C5-14 Snapgene image for the components of pMvHyg_CRISPR that are transferred by the left and right T-DNA borders $\ldots \ldots \ldots \ldots \ldots \ldots \ldots \ldots \ldots \ldots \ldots$

Figure C5-15 Microscopy images of protoplasted Microbotryum cells . . . . . . . . . 118

Figure C5-16 Snapgene image depicting the target regions and insertion construct for MVLG_05585 knockouts $\ldots \ldots \ldots \ldots \ldots \ldots \ldots \ldots \ldots \ldots \ldots$

Figure C5-17 qrt PCR expression of Cas9 in Microbotryum . . . . . . . . . . . . 122

Figure $\mathrm{C} 5-18$ PCR verification of potential Cas9 transformants $\ldots \ldots \ldots \ldots \ldots \ldots \ldots$ 


\section{CHAPTER 1}

\section{AN INTRODUCTION TO PATHOGEN/HOST COEVOLUTION AND A MODEL GENUS FOR ITS STUDY, MICROBOTRYUM}

1.1 The symbiotic relationship between plants hosts and their fungal pathogens

The symbiotic relationships between fungi and plants are ancient, originating at the dawn of terrestrial life. The transition of plants from an aquatic environment onto land was facilitated by endophytic fungi that acted as trade partners in the rhizosphere, increasing water and mineral uptake in roots of the plant host in exchange for carbon sources (Strobel 2018). Over the following hundreds of millions of years, plants and fungi both diversified; and in turn, so did their symbiotic relationships. Today there are 4 Divisions of terrestrial plants, all of which include members known to interact with fungi in some way shape or form; however, not all of these symbioses are mutually beneficial.

Fungal plant pathogens are distributed globally. In the top five agricultural crops, rice, wheat, maize, potatoes, and soybeans, fungal disease is responsible for more than 125 million tons of destroyed crops every year, enough food to feed 600 million people (Fisher et. al., 2012). This makes the study of emergent fungal pathogens and the way in which they manipulate their plant hosts, particularly important for food security on a global scale. A prime example of the dangers associated with the evolution of fungal pathogens can be observed in the recent Banana Wilt outbreak. The causative agent of this agricultural disaster is a strain of Fusarium oxysporum f. sp. Cubense, also known as the tropical race 4 or "TR4", that is currently wreaking havoc on the Cavendish banana cultivars, the variety that makes up $99 \%$ of global exports (Dita et al., 2018). While TR4 is not the first strain of Fusarium to infect banana plants, it is a particularly virulent strain that has evolved to persist in warmer climates compared to other strains that typically only emerge during cooler conditions. Understanding how these types of fungal pathogens manipulate their hosts at the molecular level, and how the two co-evolve over time, is thus imperative for targeting existing agricultural pathogens and preventing the spread of emergent strains to provide food security. 
Another prime example of the large-scale damages caused by unchecked pathogens can be see in the Puccinia genus of wheat pathogens, also known as "Wheat Rusts". These generalist fungal pathogens attack the plant and grow on the exterior, feeding on the decaying plant tissue. This, combined with their ability to overwinter within their hosts, makes the pathogen difficult to treat and often leads to massive crop loss for infected wheats, barley, and ryes. The three most common strains found in temperate region where wheats, barley, and rye crops are common include the "Stem or Black Rust", P. graminis, the "Leaf or Brown Rust", P. triticina, and the "Strip or Yellow Rust", P. stiriiformis. P. graminis is a common wheat pathogen with particularly disastrous consequences. Because wheat fields are typically clonal, once the rust establishes itself in its first host, it can quickly spread throughout the field leading to sever losses, often between 50\%$70 \%$ and sometimes destroying the entire field (USDA, 2017). Furthermore, susceptible cultivars cannot be grown in areas with recent outbreaks, as the lifecycle of the rust fungi allows it to lay dormant in the soil and overwhelm young wheat plants as they grow, increasing the operational damage caused by these rusts to the agricultural industry (USDA, 2017). While these particular rust pathogens are exceptionally detrimental to wheat cultivars in the agricultural setting, they are also known to naturally infect other plants including Rye, Barley, Foxtail Barley, Little Barley, and Russian Wildrye. This wide breadth of hosts makes P. graminis far more mobile in terms of spread, and changes within different populations of the pathogen and intermediate hosts could lead to potentially disastrous host-shifts.

\subsection{Pathogen/host coevolution and reproductive strategies}

The competitive nature of parasitism drives an intimate relationship between pathogens and their hosts as they struggle to achieve their conflicting interests. Because changes in either the pathogen or host have a direct impact on the survival of the other, the two act as reciprocal selective pressures on one another. Their allelic frequencies for genes particularly important in the infection/defense response thus follow an inverse parabola, as the success of one group ultimately selects for more fit individuals in the other, resulting in a repetitive tradeoff. This evolutionary tug-of-war was perhaps most elegantly described by Leigh Van Valen in 1973 using an excerpt from Lewis Carroll's, Through the Looking-Glass (Van Valen, 1973). In the fantasy world of Carroll's novel, the Red Queen tells Alice, “here, you see, it takes all the running you can do, to keep in the same place" (Carroll, 1971). Van Valen applied this statement to describe how pathogens 
and hosts are constantly co-evolving, a phenomenon that would eventually be referred to as the Red Queen Hypothesis.

The Red Queen Hypothesis states that in situations of uncertainty, e.g., environmental unpredictability and parasitic load, there are intense selective pressures for adaptations that provide the host with an ability to change rapidly (Bergstrom \& Dugatkin, 2011). One of the fastest ways in which the host can obtain greater diversity in their offspring is to reproduce sexually (Bergstrom \& Dugatkin, 2011). Sexual reproduction evolves in populations under the selective pressures of an unstable environment, especially pressures imposed by parasitic partners. While the origin of variation through random genetic mutation is the same for sexually and asexually reproducing organisms, sexual reproduction allows for the mixing and matching of new phenotypes, thus creating a faster spread of emergent advantageous traits and a greater depth of genetic possibilities to help escape parasitism (Auld, Tinkler, \& Tinsley, 2016) (Figure C1-1).

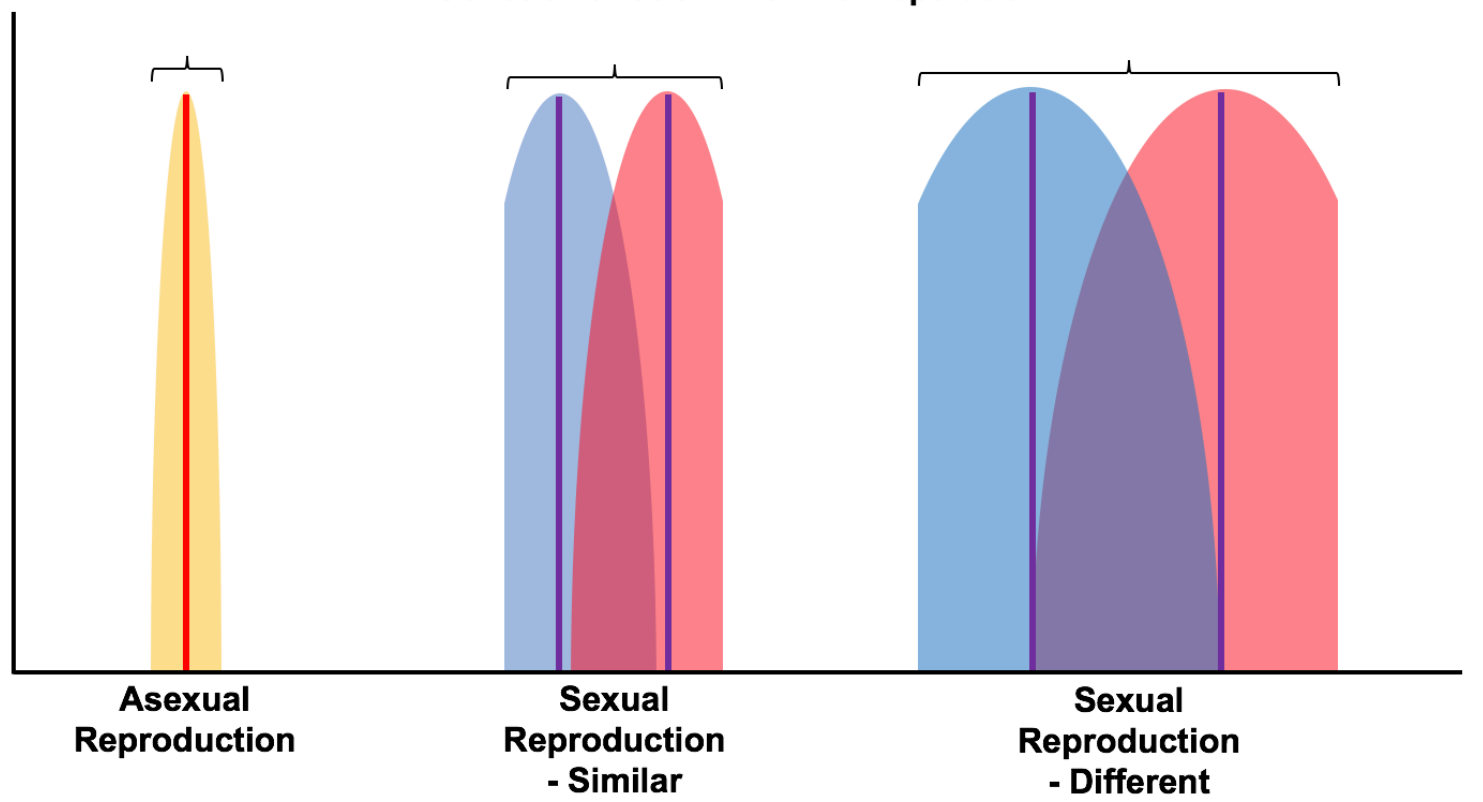

Figure C1-1 Genetic variation within a population by reproductive strategies. The y-axis represents a non-specific number of individuals within a population while the $\mathrm{x}$-axis represents various phenotypic outcomes. Asexual organisms rely on random genetic mutations for their genetic diversity and are therefore much more similar. Sexually reproducing organisms can mix and match genetic variations to their genes, indicated by the overlapping color, and therefore cover a much wider range of phenotypic possibilities in a population. 
Another advantage of sexually reproducing organisms involves the ability to purge deleterious mutation, including those that lead to physiological abnormalities that reduce the fitness of a population and those due to phenotypes that make them more susceptible to pathogens, through genetic recombination (Visser \& Elena, 2007). When deleterious mutations arise in the genetic material of a sexual organism, having multiple copies of that gene can allow for elimination of the non-functioning copy in their offspring through the mixing and matching of genes in the offspring (Visser \& Elena, 2007). Because sexually reproducing organisms each donate one copy of a gene, heterozygous individuals with non-functional copies can mate to form homozygous offspring with restored function of a beneficial gene. While this process is not guaranteed, and any other combination of offspring may arise with full or partial deletion of function in the same gene, selective pressures can drive the population back into the advantageous phenotype over time (Figure C1-2).

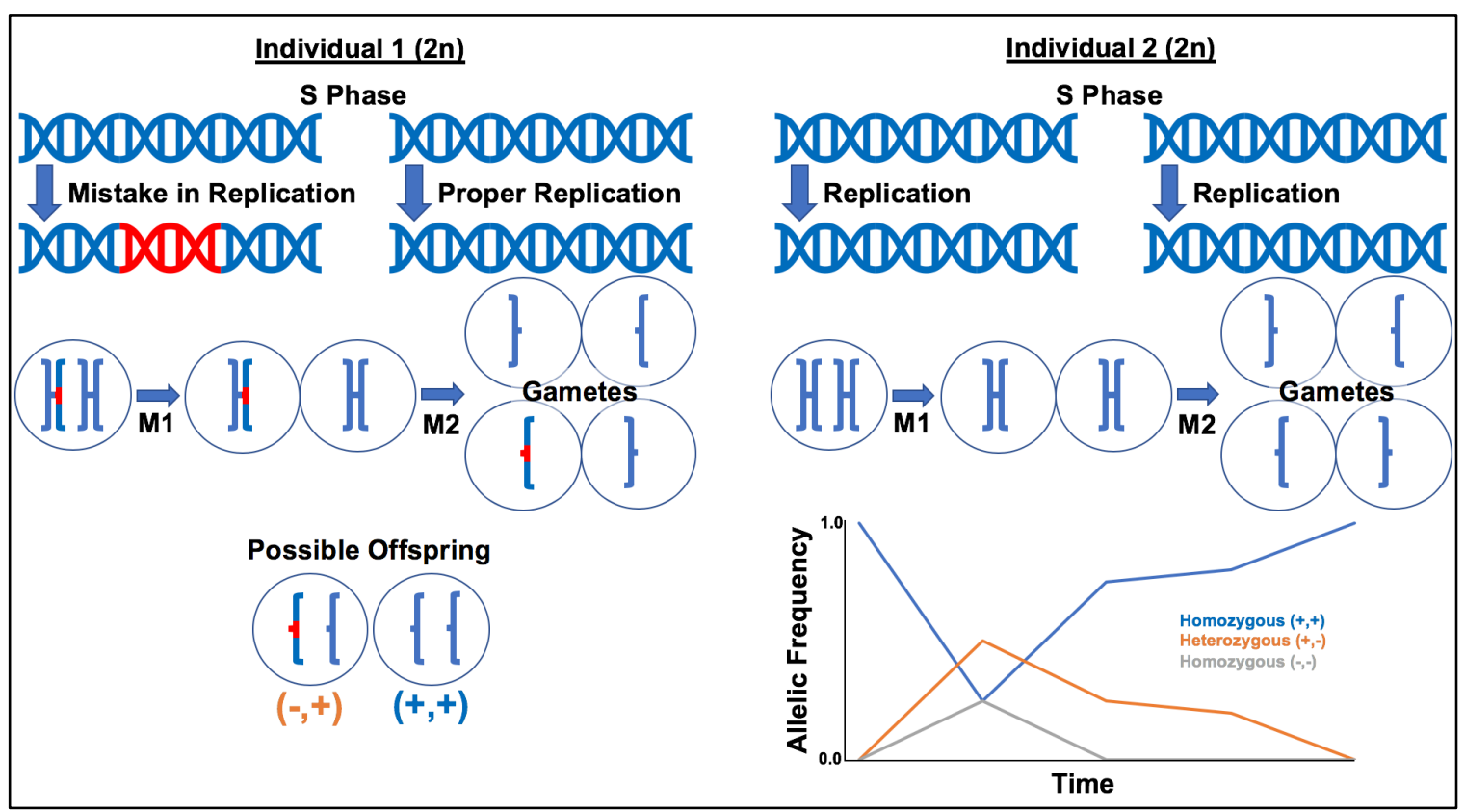

Figure C1-2 Elimination of emergent disadvantageous genes through sexually reproduction. As an emergent phenotype is selected against, genetic variability that is maintained in a population through sexual reproduction allows for return to the previous advantageous phenotype. 
Furthermore, in addition to the elimination of novel disadvantageous genotypes, sexual reproduction allows for the reversal to a previous phenotype, even if that genotype should be completely lost in a population through the process of complementation (Perlin et al., 2020). This is particularly useful in creating a moving target for genes that play a primary role in host defense responses against pathogens. Suppose alterations to a particular defense response gene allows the host to avoid inhibitors secreted by the invading pathogen. This phenotype may be so advantageous that multiple different genes may mutate to provide the same phenotype. However, over time as the host acts as a selective pressure for adaptation in the pathogen, the inhibitors secreted by the pathogen may evolve to again recognize the new form of the defense response phenotype. In a sexually reproducing population where two different gene mutations exist, let us call them Mutation A and Mutation B, sexual reproduction and homologous recombination can restore the original wild type defense response in the plant that may now be more suited for the new generation of pathogens (Figure C1-3).

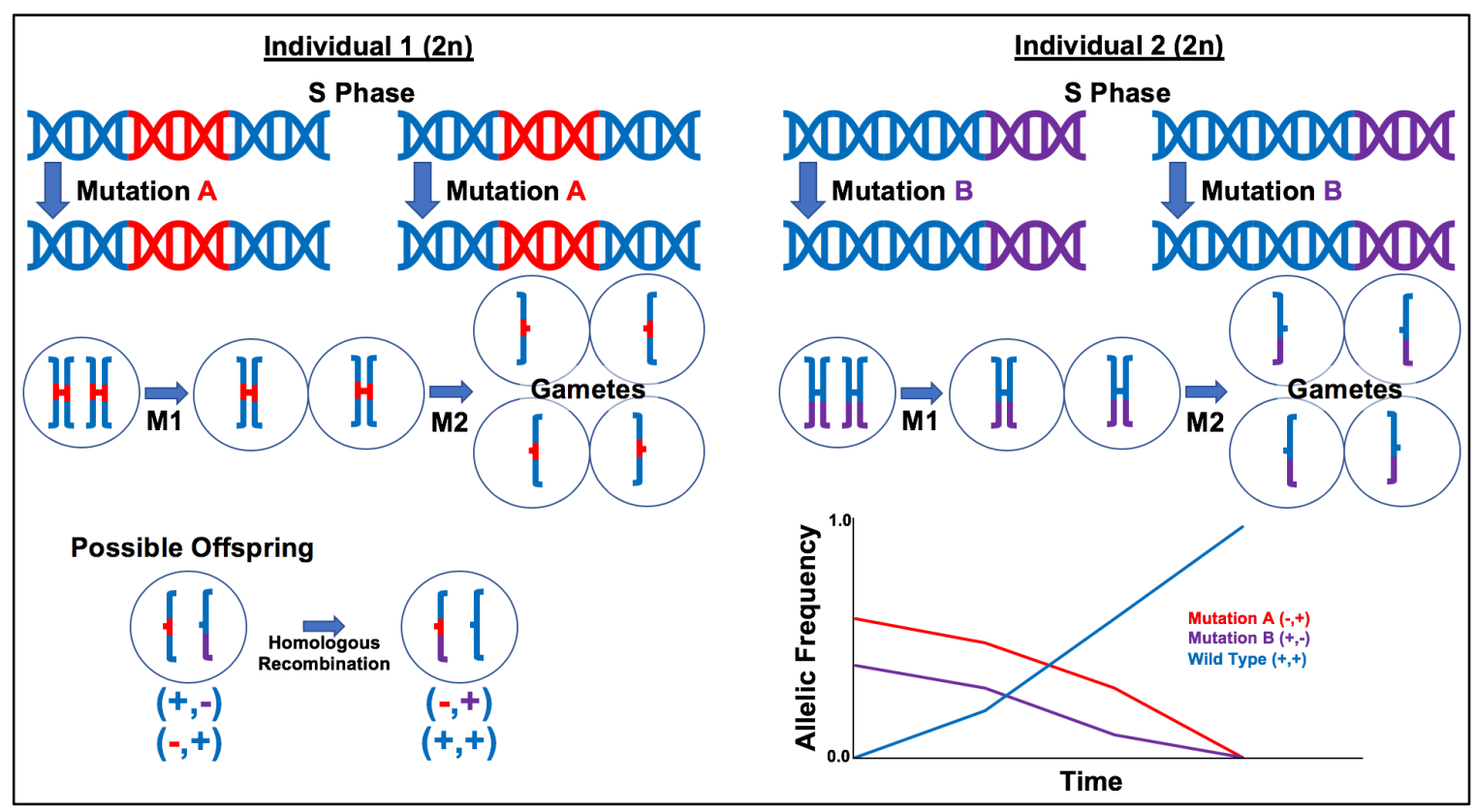

Figure C1-3 Restoration of wild type phenotype through complementation. Sexual selection can restore a population to a previous genotype through recombination of different regions of the genome previously changed in some lineages but not in others. 
Sexual reproduction therefore acts as a mechanism for rapid change, an important feature for organisms that reproduce slower than their pathogens, as one generation of hosts may deal with millions of generations of asexually reproducing pathogens.

While organisms that reproduce at such high rates can rely on the natural variations that arise through mutation during mistakes in replication, asexual populations are more vulnerable to the pile-up of deleterious changes (Bergstrom \& Dugatkin, 2011). Because many asexual populations are unable to perform genetic recombination, with the exception of horizontal gene transfer, as mutations arise in a population they cannot revert to their wild-type genotype. This phenomenon was proposed by Herman Muller and is widely coined as Muller's ratchet (Muller, 1964). Muller stated that in a population of asexual organisms, mistakes in replication lead to multiple lines of genotypes. As these genotypes all begin to accumulate mutations in a particular gene, eventually the populations will be made of several lines that all have at least 1 mutation. At this point the "ratchet" has clicked one step forward and now cannot be brought back to the original wild type phenotype, either through purifying selection through the elimination of lines with mutations or through horizontal gene transfer. The ratchet can continue to click forward in this manner, furthering the slow migration of the population towards mutation accumulation, or through the elimination of the remaining strains with fewer mutations than the rest, either through genetic drift or other random events (Bergstrom \& Dugatkin, 2011). Sexual reproduction therefore offers an advantage in the evolutionary tug-of-war between pathogens and hosts.

It is worth noting that some pathogens can also reproduce sexually, particularly fungal pathogens. Most fungal pathogens possess the capacity to both reproduce sexually or asexually, depending on the conditions in their environment (Heitman et al., 2014). This allows the pathogen to spread quickly through asexual reproduction, forming clusters of clonal lineages before sexually recombining, usually before infection of a host (Heitman et al., 2014). This bimodal approach to reproduction allows fungal pathogens to adapt much more quickly to changes in their hosts, driving rapid evolution of virulence and host range.

But sexual reproduction is not without a cost, otherwise there would be no reason asexually reproducing organisms persist. One major drawback to sexual reproduction can be observed from the gene'seye view of propagation. Richard Dawkins famously stated in his book "The Selfish Gene" that from a genes perspective, the primary function of life is to copy one's self(Dawkins, 1976). In the case of sexual vs asexual 
reproduction, asexual organisms accomplish this goal at a rate of $2 \mathrm{X}$ that of sexual organisms (Bergstrom \& Dugatkin, 2011). Because sexual reproduction requires the fusion of one gamete with a gamete from a partner, each parent is therefore only donating about half the genetic material compared to individuals from an asexual population. Furthermore, the presence of two separate mating types also imposes restrictions on population growth. In addition to a cost associated with finding a partner, the fact that a sexual population's offspring will contain a mixture of the two mating types at approximately 1:1 ratios, in most cases, means that the population will reproduce only half as fast as an asexual population in which all offspring can reproduce. This argument was made by John Maynard Smith and is referred to as the Twofold Cost of Sex (Bergstrom \& Dugatkin, 2011). When one considers these two large costs, along with the many other disadvantages than can arise from sexual reproduction, including the possibility for sexual recombination to break favorable gene combinations and the costs associated with courting a mate (Bergstrom \& Dugatkin, 2011) e.g., producing pheromones in fungal pathogens, it is a wonder that sexual reproduction exists at all. The bottom line however is tied back to the idea of the "Selfish Gene". In the presence of a persistent parasitic pathogen, by accepting the costs of sexual reproduction a population can spread more of its genetic information, albeit at a slower rate, than an asexual population completely consumed by disease.

In the case of pathogenic parasitism, the emergence of novel pathogens with a strong advantage on a host can lead to the rapid spread of a new strain through a host population with little diversity (Bergstrom \& Dugatkin, 2011). We have seen many examples of this phenomenon in the world's agricultural industry, including the aforementioned Cavendish banana and wheat cultivars, as artificial selection practices favor monocultures of the largest. Because these crops are genetic replicas of one another, and therefore have little genetic diversity, once a pathogen evolves to successfully infect one there is no impediment to successful infection of the rest of the population as well. The same can be thought true for asexually reproducing populations. On the other hand, heirloom cultivars of crops which are often pollinated through open air pollination contain a much higher degree of genetic variation. While this diversity leads to a large variation in crop sizes, they are in turn are less affected by emergent disease (Dwivedi, Goldman, and Ortiz, 2019). Research into the advantage of heirloom practices demonstrated that crops were more resistant to soilborne insects, chronic disease, and exhibited greater stress tolerance compared to monoculture cultivars (Dwivedi, Goldman, and Ortiz, 2019). To continue the metaphor from through the Looking-Glass, although genetic 
diversity usually does not allow a host to outrun their pathogens, the genetic diversity does allow them to create a moving target, or allows them to keep running to stay in the same place as Lewis Carroll would say, rather than being completely overwhelmed by the pathogen (Bergstrom \& Dugatkin, 2011; Carroll, 1971). The Red Queen Hypothesis therefore accurately proposes that recombination of genetic material is selected for in hosts to increase their genetic diversity in order to help them out-pace their pathogens, and the genetic diversity that is created through sexual reproduction lays the ground work for an evolutionary tug-of-war between the pathogen and the host (Morran et al., 2011).

1.3 The role of secretory proteins in pathogen/host coevolution

While even sexually producing populations can share a wide degree of similarity in physical appearance, when we consider the types of changes that are going on at the molecular level within a population of pathogens and hosts we find that chemical compounds and proteins that play a role in cell communication and recognition/repression are constantly adapting to changes that arise in the new generations of hosts and pathogens, a phenomenon that over time can lead to host specialization. The success of a fungal pathogen infecting and otherwise manipulating their hosts depends heavily on their arsenal of secreted compounds. As saprophytic chemotrophs, fungi interact with the world around them through the secretion of compounds that play a variety of roles from external digestion to defense against predation (Urry et al., 2017). In the case of fungal pathogens, these compounds can play a role in host penetration, host manipulation, eliminating competing fungi or bacteria, breaking down carbon sources for food, and identification of potential mating partners. While some fungal pathogens are opportunistic and grow on dying plants, others live on or inside living hosts, utilizing secreted proteins that can dissolve cellulose to enter the cell wall of the plant host, as well as proteins that can moderate the plant host's defense response through suppression of signal transduction pathways or gene expression (Rep, 2005) and manipulate the host through modification to hormonal pathways (Rabe et al., 2013).

While all fungal pathogens manipulate their hosts to some degree through the secretion of various proteins and other compounds, their approach to doing so can vary dramatically. Of these different approaches, there are three main strategies; necrotrophic pathogens employ a particularly destructive approach of overwhelming the host and causing extensive necrotic damage to tissues killing the host in the 
process, biotrophic pathogens on the other hand pursue a much more subtle strategy preferring to establish a long-term feeding relationship with their hosts by living in or on their hosts without killing them, and saprophytic pathogens forgo battling with hosts altogether, preferring to colonize and consume dead material instead. Regardless of strategy, each lifestyle requires the secretion of proteins to manage their hosts, perform external digestion, and/or fend off other opportunistic microorganisms.

Plants also utilize the secretion of proteins and other secondary metabolites in the arms race against their pathogens (Vincent, Rafiqui, \& Job, 2019). Upon detection of a fungal pathogen, plants can utilize both the traditional ER-Golgi mediated secretion of upregulated proteins, and small extracellular vesicles for the secretion of leaderless secretory proteins and other secondary metabolites for combat in the extracellular space (Vincent, Rafiqui, \& Job, 2019). If we consider the coevolution between the secreted proteins of the pathogen and the secreted proteins of the host an evolutionary arms race, then the battlefront is the apoplast of the host, where fungal pathogens try to break through and the plant host hunker down to defend the line. The apoplast of a plant is well fortified by the open extracellular space and its cell wall, comprised of complex networks of polysaccharide polymers and glycoproteins. In addition, the apoplastic space contains apoplastic fluid circulating throughout the cell wall and facilitating both fast communication between cells and delivering defense proteins (Delaunois et al., 2014). Signals facilitated by the apoplastic space allows the host to recognize microbial-associated molecular patterns (MAMPs) or damage-associated molecular patterns (DAMPs), two categories of Pattern Recognition Receptors (PPRs), to begin their defense response (Delaunois et al., 2014). This ultimately makes the apoplastic space the front line in the battle to determine who is likely to win the war. Because higher plants are constantly interacting with both mutualistic and parasitic microbes, they have evolved to recognize a large range of different MAMPs for a large variety of different pathogenic species, some examples of which are listed in Table C1-1. 
Table C1-1. Examples of Microbe-Associated Molecular Patterns

\begin{tabular}{|l|c|c|}
\hline \multicolumn{1}{|c|}{ MAMP Signal } & Organisms Detected & Reference \\
\hline Eicosapolyenoic acids & Oomycetes & (Savchenko et al., 2010) \\
\hline$\beta$-glucans & Fungi & (Wlarzynski et al., 2000) \\
\hline peptidoglycans & Bacteria & (Erbs and Newmann, 2012) \\
\hline lipopolysaccharides & Gram Negative Bacteria & (Sanchez et al., 2007) \\
\hline rhamnolipids & Bacteria & (Miya et al., 2007) \\
\hline Chitin oligomers & Fungi & \\
\hline
\end{tabular}

Because evolution selects for pathogens which are able to secrete effector proteins which specifically block the host proteins responsible for the recognition of MAMPs, plants have also evolved effector-triggered immunity, a fast-tracked version of defense response signaling that operates by directly recognizing these anti-MAMP effectors secreted by their pathogens (Delaunois et al., 2014). The response to recognition of virulence factors from a known pathogen elicits a much stronger immune response in the host, often triggering compartmentalization of infected cells through the closure of plasmodesmata and programmed localized cell death to prevent the spread of the pathogen (Tsuda and Katagiri, 2010). This process is accomplished through the activation of MAPK kinases, which release reactive oxygen species within minutes of detecting a foreign effector (Delaunois et al., 2014). It is here that we can begin to see the evolutionary arms race materialize at the molecular level as pathogens act on the host to evolve better recognition of pathogen secreted proteins, and in turn, hosts act as selective pressure for more discrete inhibition of host responses in the pathogens.

To win a battle in the ongoing evolutionary arms race, pathogens must secrete a variety of compounds or effectors to suppress PPR-mediated defense responses and otherwise manipulate their host. Many host-specific groups of pathogens have sets of proteins with yet unknown roles, roles that are vital to the infection of a particular species or genus of hosts. These fungal effector proteins are often small $(<250$ amino acids), have no know Pfam domain (regions of amino acid sequences shared amongst various protein families indicative of a particular known protein function), and are often limited in their phylogenetic distribution due to rapid evolutionary pressures imposed by the pathogen/host arms race (Rep, 2005). Interestingly, these small secreted proteins vital for infection are also highly likely to stimulate the host 
immune system, further supporting the idea of coevolution between the pathogen and host (Rep, 2005). However, despite the absences of a Pfam domain or other known highly conserved function across the wide database of available pathogen genomes, small secreted proteins that play a role in host pathogenicity are more likely to be shared amongst closely related lineages that parasitize similar hosts. This combination of preservation of proteins amongst closely related pathogens with no known Pfam or GO terms a good place to start for the identification of host-specific effectors.

Through a decade of molecular genetics and bioinformatic analyses made possible by the everexpanding accessibility and ease of genetic sequencing, many novel fungal effectors have been identified. These effectors either promote the virulence of fungal pathogens or suppress defense responses, allowing the fungus to colonize the host. Examples of well conserved effectors found across fungal pathogens include secretory lipases, effectors that inhibit plant-mediated immunity response through inhibition of callose formation (Marzin et al., 2016), and pectinesterases, which act to modify cell wall composition and allow for penetration into the plant host (Blümke et al., 2014). While these types of effectors are heavily conserved amongst plant pathogens, coevolution and genetic drift lead to a wide diversity of effector amino acid sequences. Therefore, using bioinformatics to identify regions of the amino acid sequences that may be more conserved amongst closely related groups, such as activation domains which may be under selective pressures due to their importance for protein function, can be useful to recognize conserved features among various effector families. Furthermore, a lack of identifiable Pfam domain or GO term can indicate a unique function for effectors in a system that result from the intimate coevolutionary relationship between the pathogen and its host.

Once established inside the plant, fungal pathogens can manipulate their hosts through the modification, or even the secretion of synthetic versions of plant hormones (Ma \& Ma, 2016). Several recent studies have identified a variety of phytohormones that play a major role in regulating plant-microbe interaction (Vincent et al., 2020). These include the "Big Five" plant hormones, auxin, gibberellin, cytokinin, ethylene, and abscisic acid (Ma \& Ma, 2016), as well as others such as salicylic acid, and jasmonic acid (Vincewnt et al., 2020). While some of these hormones are disrupted due to their direct role in combating the spread of the pathogen in the hosts, e.g., abscisic acid exhibits antifungal properties (Khedr et al., 2018), other plant hormones may be manipulated to induce more preferable conditions in the hosts, e.g., through the 
induction of galls via modulation of indole-3-acetic acid and cytokinin (Davies et al., 2005; Mizoi et al., 2012). Furthermore, by manipulating the plant hormones pathogens can affect the development and growth of plant tissue, a process that can aid in the spread of disease to new hosts (Ma \& Ma, 2016).

As the molecular arms race plays out over evolutionary time, slow divergence of hosts due to reproductive barriers can lead to slight changes in the host-specific secreted proteins of the fungal pathogen isolates. These changes can accumulate and lead to speciation events in the fungi themselves. As the amino acid sequence for a particular protein changes rapidly to keep up with evolving host defense response in a geographically isolated population, local adaptation can lead to different changes that over time can cause the fungi to be unable to infect hosts outside of their population. This can lead to interesting changes to conserved core secreted proteins or to the addition of entirely novel proteins to the secretome of these fungi. Over time, these changes amplify host-specificity of these fungi, and local adaptations can lead to postzygotic barriers in fungi of different populations. There are then direct selective pressures for the plant to be able to quickly identify pathogens and direct inverse selective pressure for pathogens to be able to quickly mediate plant defense responses (Figure C1-4).

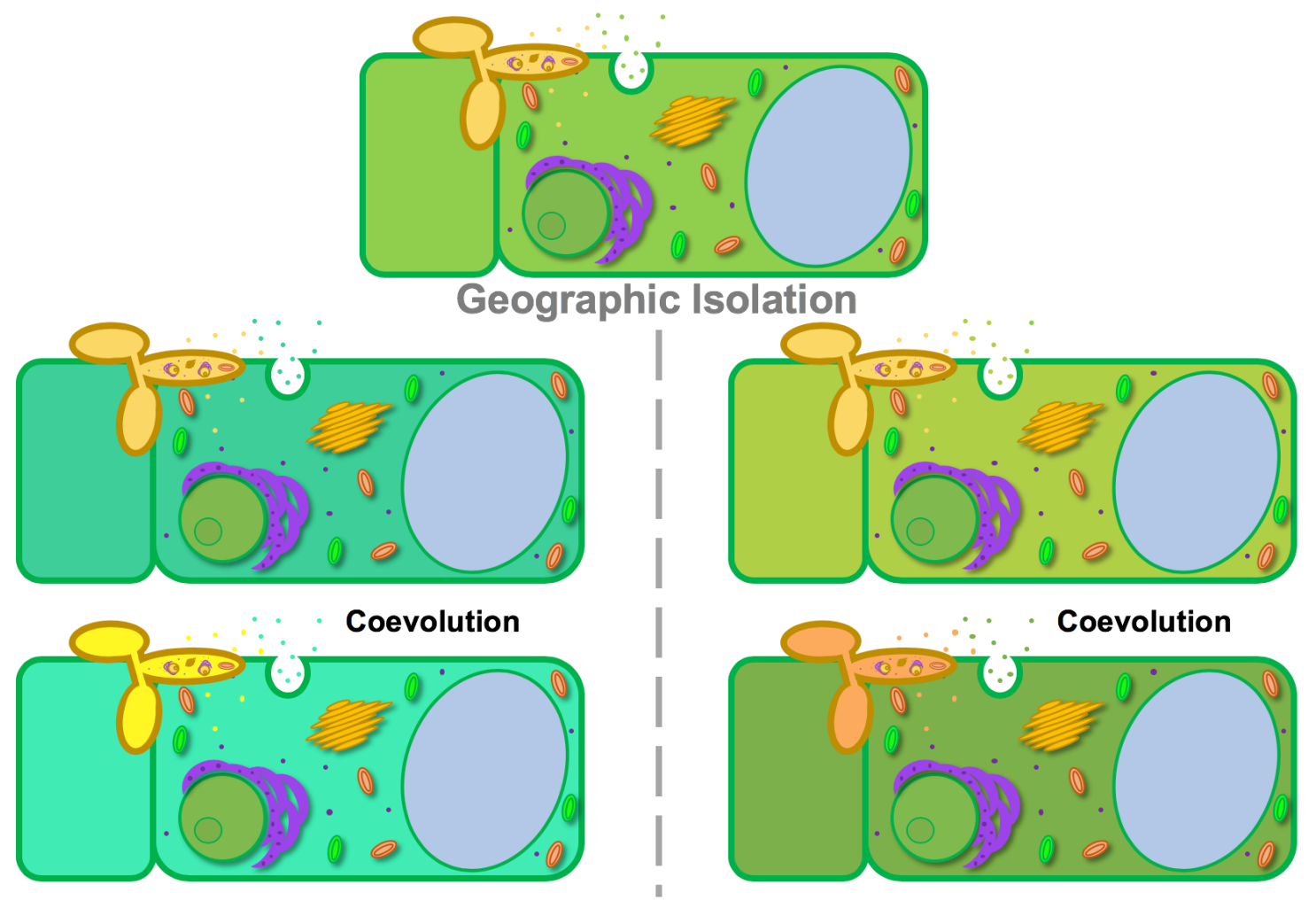


Figure C1-4 A Model for secreted protein molecular arms race. How geographic isolation of infected hosts can lead to coevolution and speciation in both the host and the pathogen.

\subsection{The anther-smut fungi, Microbotryum}

As pathogens become more and more specialized to the genetic diversity found within their population of hosts, limited gene flow between populations, either due to geographical or other reproductive barriers, can result in intense host-specificity. Therefore, speciation events in the host can subsequently lead to speciation events in their pathogens (Figure C1-4). In systems with pathogen-host coevolution, phylogeny between the two often represent a near mirror image. Such is the case for the anther-smut pathogen species complex, Microbotryum violaceum and their Caryophyllaceae hosts (Figure C1-5 from Hartmann et al., 2019).

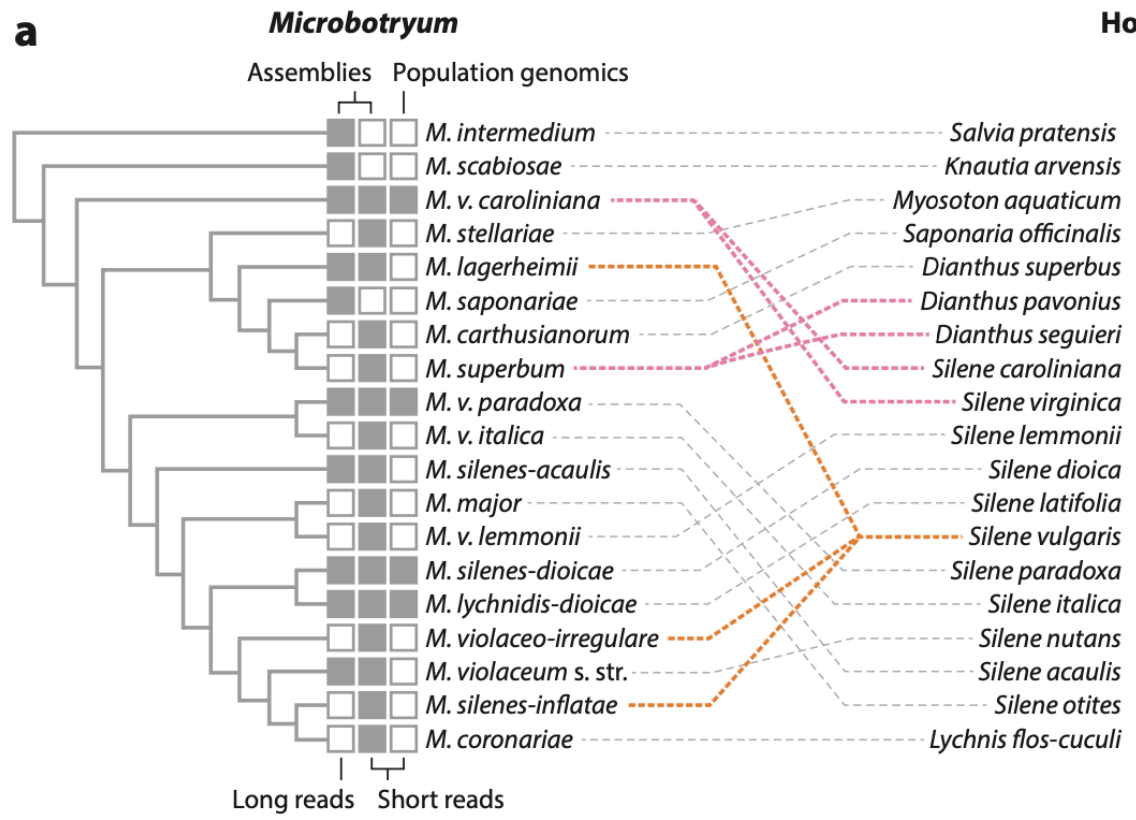

\section{Hosts}
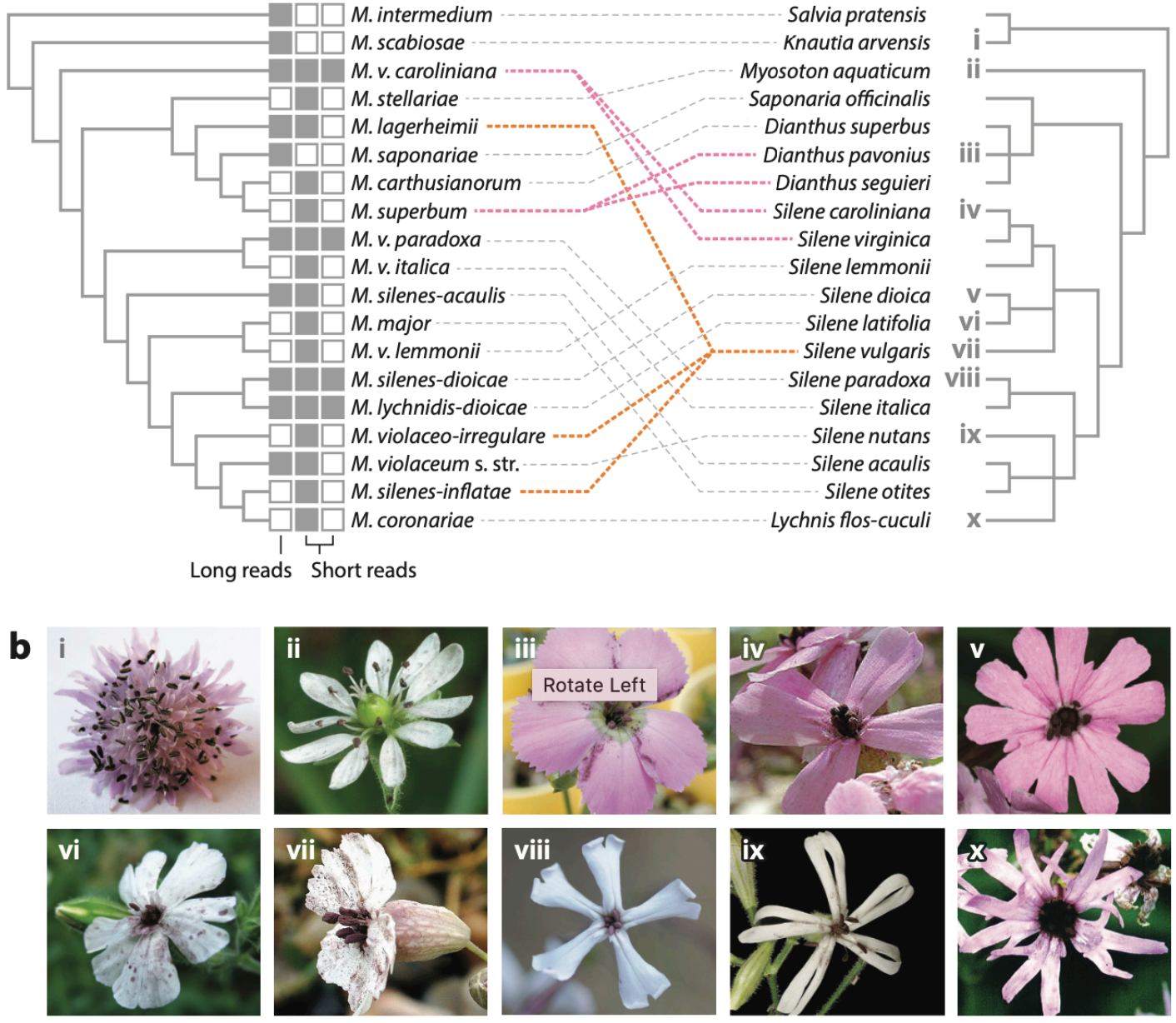
Figure C1-5 Comparison of plant and fungal phylogenies using one strain per host species. The phylogeny of Microbotryum species, right, and their Caryophyllaceae hosts, left demonstrate the coevolution and host-shifts observed in the pathogen/host pairings. Image is from BMC Evolutionary Biology with permission from Hartmann et al., 2019.

Upon its initial discovery, the Microbotryum violaceum species complex was originally described as a monophyletic generalist pathogen of the Ustilagoinomycotina lineage, Ustilago violacea, that parasitized various members of the Caryophyllaceae family of flowers (Baker, 1947; Fischer and Holton, 1957). However, morphological studies and infection assays have since demonstrated that the Microbotryum genus is a collection of separate species denoted by their intense host-specificity to one or two hosts. The life cycle of the fungus begins and ends in the anthers of their hosts. Many nocturnal moths and diurnal hoverflies are known to pollinate members of the Silene genus (Jürgens, Witt, \& Gottsberger 1996), carrying the spores of their corresponding Microbotryum species to new hosts. In the particularly well studied interaction, that of Microbotryum lychnidis-dioicae on Silene latifolia, the nocturnal moth Hadena bicruris is one of the main pollinators driving the spread of M. lychnidis-dioicae (Jürgens, Witt, \& Gottsberger 1996). Of interest, there does not appear to be discrimination by the H. bicruris for flowers uninfected with Microbotryum spores. While there is little research on the topic, it does beg the questions if the fungal spores are attracting the pollinators via chemical mimicry or otherwise fooling these insects in order to improve propagation. Once a teliospore of the fungus is deposited on a new host, germination occurs and meiosis results in the production of the yeast-like stage of the fungi, haploid cells known as sporidia. In nutrient rich conditions, such as those found in the nectar of the host, these fungal sporidia will continue to reproduce asexually through budding (Schäfer et al., 2010) (Figure C1-6-2). As the flowering season ends in the fall, the available carbon depletes and the fungal cells of opposite mating types form conjugation tubes, mating and forming the infectious dikaryotic filament. This dikaryotic hyphal structure penetrates the plant cell wall (Figure C1-6-4) and the fungus migrates to the roots where it will overwinter with the host until the spring. In the following spring, as the plant bolts and flowers begin to form, the fungus migrates back up to the anthers of developing flowers where the infection process is completed Separate fungal nuclei fuse to form diploid teliospores which are then deposited on the anther in of aborted pollen for transport by unsuspecting pollinators. 


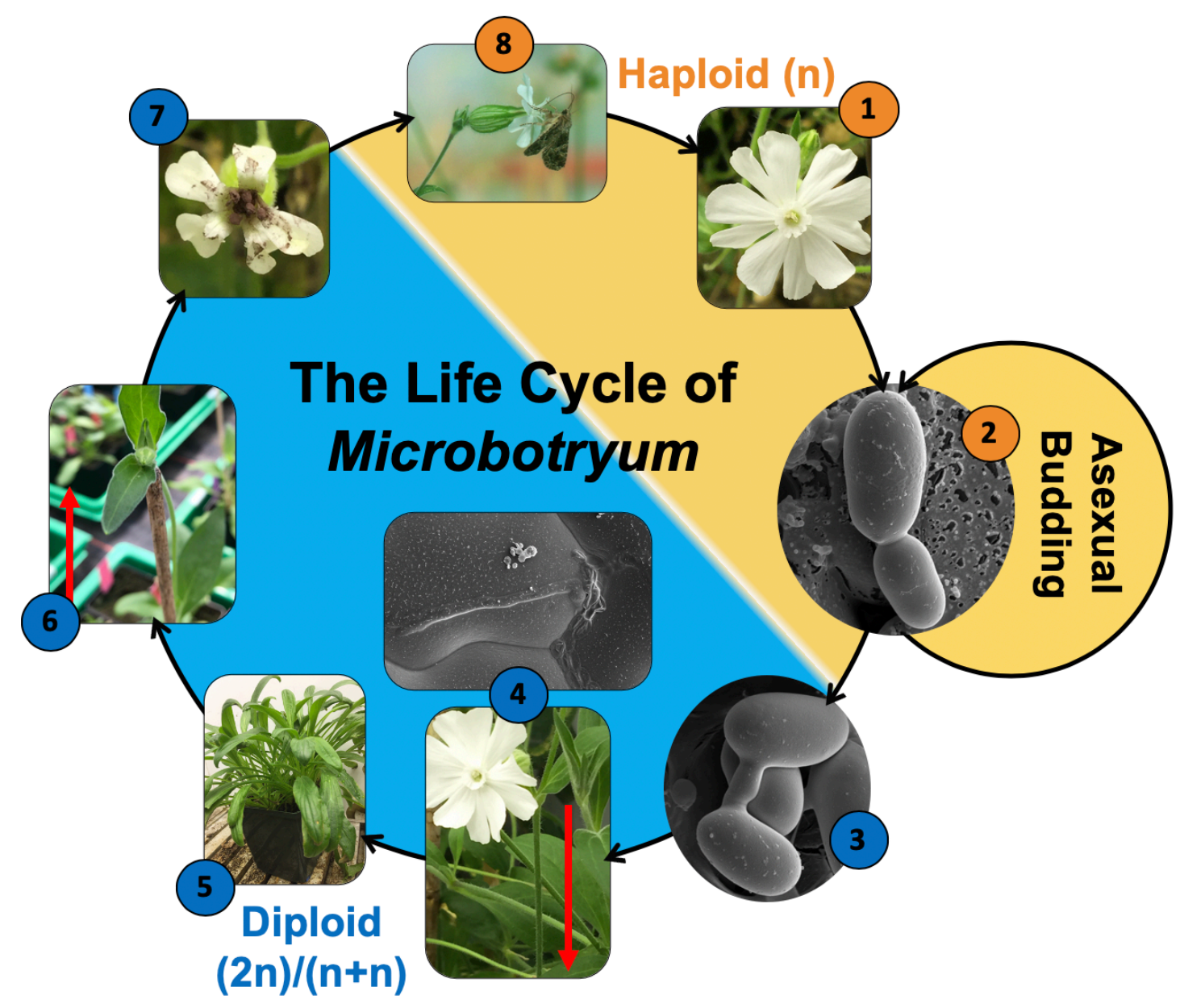

Figure C1-6 The life cycle of Microbotryum, demonstrated with M. lychnidis-dioicae. 1) Pollinators bring teliospores to new host flowers. 2) The teliospores germinate and undergo meiosis to produce haploid sporidia capable of asexual budding. 3) These yeast-like cells find compatible mating types and form a conjugation tube. 4) An infectious dikaryotic filament is formed and penetrates the plant. 5) The dikaryotic fungus travels to the roots of the hosts where they overwinter with the plant. 6) In the spring, the dikaryotic fungus travels to the developing anthers during bolting. 7) Karyogamy result in generation of fungal teliospores located on the anthers of the host. 8) A pollinator picks up fungal spores and transports them to a new host.

When infection studies are performed in a controlled lab setting via inoculation of seedlings with suspensions of both fungal mating types, infection is systemic and successfully infected plants will contain teliospores in all flowers; however, it has been observed in the natural setting that plants can have both infected and non- 
infected flowers. This partial infection in nature may play an important role in ensuring propagation of the host species for future generations of Microbotryum, a sophisticated approach for any pathogenic life cycle, as systemic infection such as those seen in laboratory studies would even result in infected flowers produced by vegetative runners. When infected, Silene hosts have demonstrated an increased rate of fluorescence, a potentially advantageous host manipulation for the dissemination of more fungal spores. Furthermore, some species of Microbotryum have been observed displaying yet another adaptation for the infection of dioecious hosts, such as S. latifolia, where infection of a female flower leads to abortion of the flower ovary and induced production of pseudoanther for placement of fungal spores (Toh et al., 2018). While a mechanism for this unique phenomenon is not yet identified, the ability of the pathogen to manipulate the development and growth of its hosts is much in line with previously identified hormonal pathway manipulations in other fungal pathogen/plant host pairings (Ma \& Ma, 2016).

The intense host specificity, together with the ability of Microbotryum species to infiltrate its host undetected and manipulate the reproductive chemistry of female flowers, suggests that each Microbotryum species utilizes a unique portfolio of secreted compounds during pathogenicity, a starting point to understanding pathogen/host coevolution between the many members of the Microbotryum genus and their many hosts over time. This dissertation will explore the role of Microbotryum effectors in host-specialization and manipulation by implementing both a bioinformatic approach to identify and compare the secretomes of closely related Microbotryum species and molecular genetics techniques to characterize the role of a few representatives of the small secreted proteins. Secretomic comparisons, discussed further in Chapter 2, were first performed on three closely related Microbotryum species, two sister species with capacity for cross infection of each other's host, M. lychnidis-dioicae and M. silenes-dioicae, and one more distantly related species for which infection in the hosts of the two sister-species is not observed, M. violaceum var paradoxa. From this, a list of conserved and species-specific small secreted proteins were identified. Next, in Chapter 3 , this dissertation introduces the characterization of a core secreted protein through a series of molecular genetic tests to demonstrate secretion of the protein, identify physiological localization outside of the cell, and identify the target within the host. Chapter 4 then outlines the role of species-specific proteins in host specialization through infection studies utilizing heterologous expression of species-specific small secreted proteins from each sister species of Microbotryum. Finally, Chapter 5 of this dissertation addresses the work 
done to implement a reliable CRISPR-Cas9 approach to generating site-specific gene knockouts in the Microbotryum genus in order to further characterize the identified list of both core and species specific effectors for future research. 


\section{CHAPTER 2}

\section{IDENTIFICATION OF CORE AND UNIQUE SECRETED PROTEINS THROUGH COMPARATIVE SECRETOMICS}

\section{Introduction}

Host specialization is a phenomenon well documented in many fungal pathogen/plant host systems (SánchezVallet et al. 2018), which most often occurs through host shifts (de Vienne et al. 2013). The ability to infect a new host is determined by the protein-protein interactions that occur at the pathogen/host interphase. For pathogens to be successful, they must not only be able to colonize the host, but must also work around a gauntlet of host defense responses, as well as manipulate the host to their advantage. Pathogens accomplish these ends through the deployment of many secreted effectors (Lanver et al. 2017; Anderson et al. 2010; Whisson et al. 2007).

It has been understood for several decades that plant pathogens utilize secreted effectors to infect their hosts (Albersheim and Anderson, 1971; Sánchez-Vallet et al., 2018), including the maize pathogen member of the "smut fungi", Ustilago maydis (Lanver et al. 2017). To defend against these pathogens, plants continuously evolve to recognize pathogen-associated molecular patterns and trigger a variety of immune responses (Jones and Dangl, 2006). Reciprocally, there is an ongoing selective pressure for plant pathogens to adapt to their host by developing new effectors, or otherwise alter the composition of their secretomes, to evade detection and find new ways to manipulate the host to their advantage. Secretomes can thus evolve rapidly, not only during host shift events but also due to intra-specific coevolution (Meile et al. 2018). It is, however, still unclear whether changes in secretomes leading to host specialization and local adaptation primarily involve effector gene gains/losses or changes in their sequences. Repeat-induced point mutations (RIP) is a fungal defense mechanism against transposable elements that has been suggested to play a role in effector diversification in fungi harboring effectors in regions rich in repetitive elements (Fudal et al. 2009; Van de Wouw et al. 2010). RIP indeed acts via mutations of repeated sequences at specific target sites and can "leak" on neighbor genes (Fudal et al. 2009; Van de Wouw et al. 2010). 
Host specialization following host shift is particularly common in the fungal pathogen species complex Microbotryum violaceum (Refrégier et al. 2008). Microbotryum species are basidiomycete smut fungi that complete their life cycle in the anthers of their respective host plants, replacing the pollen with their own fungal spores (Schäfer et al. 2010). Originally described as a single species, these "anther smuts" are now understood to represent a complex of species (Perlin et al. 1997; Le Gac et al. 2007a), most being highly specific to particular species of the Caryophyllaceae family, also known as "pinks" (Hood et al. 2010). Intra-specific coevolution has also been suggested to occur based on local adaptation patterns, where host plants were more resistant to their local sympatric anther-smut pathogen than to those from geographically distant populations of the same species (Kaltz et al. 1999, Feurtey et al. 2016).

To infect their hosts, Microbotryum fungi, like many other plant pathogens, employ an array of effector proteins to block plant immune response and otherwise manipulate the host during infection (Perlin et al. 2015; Kuppireddy et al. 2017). While the specificity of the various Microbotryum species to their corresponding host plants has been extensively described (Hood et al. 2010; Le Gac et al. 2007; de Vienne et al. 2009), the molecular basis for host specialization and coevolution within the complex has just recently begun to be explored (Hartmann et al. 2018; Badouin et al. 2017; Aguileta et al. 2010). Understanding the changes that have occurred in the secretomes of these host-specific species will broaden our understanding of the mechanisms behind coevolution, host-shifts and emergent diseases. Furthermore, Microbotryum species offer a unique model system to study host shifts and specialization, with multiple host-specific and closely related pathogens (Hartmann et al. 2019), which is not often the case in agriculturally propagated crops.

To test whether host-specific or locally-adapted closely-related pathogens mainly differed in their secretomes by gene gains/losses or by rapid evolution of shared effectors, we compared the secretomes of three Microbotryum species, two sister species, M. lychnidis-dioicae and M. silenes-dioicae, and a more distantly related relative, $M$. violaceum var paradoxa. We sought to identify sets of core secreted proteins (i.e., orthologous genes encoding secreted proteins shared by all species), that likely play a major role in the pathogenicity of the species complex as a whole. We also sought to identify species-specific effectors and effectors evolving under positive selection and highly expressed in planta, thus perhaps involved in host specificity. To further our understanding of coevolution and local adaptation, we compared the secretomes 
of two M. lychnidis-dioicae strains collected from geographically distant populations belonging to distinct genetic clusters that have shown contrasted infection patterns consistent with plant local adaptation (Feurtey et al. 2016). We also investigated whether the most frequent changes among host-specific species or locallyadapted clusters involved mostly the gain/loss of secreted proteins or the diversification of shared proteins. As RIP-like footprints have been detected in Microbotryum fungi (Hood et al. 2005), we also tested whether sequence divergence in genes under positive selection and/or in genes encoding secreted proteins could have been facilitated by RIP.

\section{Materials and Methods}

\subsection{Comparative genomics}

To analyze the relationship between various predicted effectors, we performed genomic analyses on the following available genomes, obtained using Pacific Bioscience (PacBio) single molecule real time sequencing: GCA_900015465.1 for M. lychnidis-dioicae Lamole a1 (Italy) (Branco et al., 2017), GCA_900015495.1 for M. violaceum var paradoxa from Silene paradoxa 1252 a 1 (Branco et al., 2018), and QPIF00000000 for M. silenes-dioicae $1303 \mathrm{a}_{2}$ (Branco et al. 2017). These genomes were selected for comparison due to their relationship to one another; M. lychnidis-dioicae strains and M. silenes-dioicae are sister species, able to infect one another's host in the greenhouse, although to a lesser degree than their natural host (Gibson et al. 2014) and very little in natural populations (Gladieux et al. 2011), while M. violaceum var paradoxa serves as an outgroup, unable to infect either of the sister species' hosts or vice versa (de Vienne et al. 2009).

In total, we used eight sequence-based prediction tools to identify potential effectors by searching each genome for genes with hallmarks for secretion and without conflicting cellular localization predictions. The initial list of putative secreted proteins (SPs) were generated by running the entire genomes through SignalP 4.0 (Petersen et al., 2011). In order to increase the stringency of this analysis, the SPs must then have passed the following criteria to rule out potential localization or retention in various membranes within or on the cell, similar to the previously published protocol for M. lychnidis-dioicae (Perlin et al. 2015). Potential transmembrane domains were predicted with TMHMM (Krogh et al., 2001) and Phobius (Käll et al., 2007).

Only gene models with none or a single transmembrane domain prediction overlapping the signal peptide 
prediction were considered further (Perlin et al., 2015; Petersen et al., 2011). Prosite was used to screen for predicted endoplasmic reticulum retention signals, while PredGPI (Pierloni et al., 2008) was used to screen for potential glycosylphosphatidylinositol anchors, and NucPred (Bramaier et al., 2008) was used to screen for nuclear localization signals in the predicted protein (Figure 1).

Gene models predicted to be secreted and without conflicting localization predictions (i.e., negative for transmembrane domains, endoplasmic reticulum retention, GPI-anchoring, and nuclear localization) were further screened using additional criteria to identify strong predictive footprints of secretion in the signal peptide region. To qualify as a SP, the candidates must also have passed stringent cutoff values for secretion, listed in Figure 1, for at least three of the following four tests: a predicted secretion signal by TargetP (Emanuelsson et al., 2000), a D-score of greater than 0.43 for the neural network [NN], a secretion probability of greater than 0.8 for the hidden Markov model [HMM] from SignalP3.0, and predicted secretion by Phobius.

We searched the resulting putative SPs among the orthologous groups reconstructed previously (Branco et al., 2018). Briefly, the orthologous groups were obtained using mcl (van Dongen, 2000) to cluster high-scoring blastp matches between all gene models predicted in 15 haploid genomes from eight Microbotryum species, previously parsed with orthAgogue (Ekseth et al., 2014). We classified a predicted $\mathrm{SP}$ as a species-specific SP if there was no ortholog in two of the species being considered. For predicted SP belonging to orthologous groups, we distinguished between species-specific, two- or three-way orthologous groups (i.e., predicted as SP in a single, in two or in three species, respectively) and between orthologous groups composed exclusively by predicted SP (SP-only) and those containing at least one gene model not predicted as SP (SP-mixed). We defined the "core secretome" as the full set of predicted SPs belonging to SP-only three-way orthologous groups (i.e., present and predicted as SPs in all three species). Conversely, we defined as "accessory secretome" the predicted SPs that were either species-specific or belonged to SPmixed or two-way SP-only orthologous groups (i.e., were not present in all species or not predicted as SP in all species; Figure 2). Together, the core and accessory secretomes make up the "pan-secretome", i.e., the full set of predicted SP in all species considered. 


\subsection{Pfam domain annotation}

We searched Pfam release 32 (El Gebali et al., 2019) against the translated gene models of all predicted SP and their homologs with hmmsearch from the hmmer 3.1b1 suite (http://hmmer.org). Hits with an E-value smaller than 1e-3 were considered significant. The results were then categorized by size as well as presence/absence of a predicted Pfam domain (supplemental file SF1 from Beckerson et al., 2019).

\subsection{Signal peptide clustering and experimental validation}

We clustered the predicted signal peptide sequences with CD-HIT (Huang et al., 2010) allowing for up to five amino acid differences (non default options: -c $0.75-15$ ). We tested if predicted signal peptides could direct the secretion of the Suc2 invertase employing a yeast-based secretion trap method (Lee and Rose, 2012; Kuppireddy et al. 2017). Six signal-peptide encoding sequences, as determined by SignalP 4.1 software, were amplified by PCR. Standard PCR cycle was used with initial denaturation set at $94{ }^{\circ} \mathrm{C}$ for 4 min and 35 cycles of $94{ }^{\circ} \mathrm{C}$ for $30 \mathrm{~s}, 60{ }^{\circ} \mathrm{C}$ for $30 \mathrm{~s}$ and $72{ }^{\circ} \mathrm{C}$ for $30 \mathrm{~s}$ and final extension time of $5 \mathrm{~min}$ at $72{ }^{\circ} \mathrm{C}$. The purified fragments were then subcloned into a TOPO vector using an Invitrogen TOPO TA Cloning ${ }^{\circledR}$ kit, and subjected to restriction digestion with Eco RI and Not I enzymes. The digested fragments were then purified and cloned into the pYST-0 vector, upstream and in-frame with an invertase coding sequence, SUC2. The presence of each signal peptide encoded in-frame with the SUC2 coding region was confirmed by DNA sequencing (Eurofins, Louisville, KY).

Invertase deficient (suc2-) Sacchromyces cerevisiae strain (SEY 6210 - MATaleu2-3, 112 ura3-52 his- $\Delta 200$ trp1- $\Delta 901$ lys2-801 suc2- $\Delta 9$ GAL) cells were transformed with the constructs using the FrozenEZ Yeast transformation II kit ${ }^{\mathrm{TM}}$ from Zymo Research. Cells were then suspended in water and spread onto synthetic drop (SD) out, $\mathrm{SD} /$-Leu (Clontech) selection plates with either sucrose as the sole carbon source or

glucose as a control. Resulting colonies from the sucrose plates were grown overnight in $3 \mathrm{ml}$ of $\mathrm{SD} /-\mathrm{Leu}$ broth with sucrose and $10 \mu \mathrm{L}$ of undiluted, 10 -fold dilutions, and 100-fold dilutions were spotted onto $\mathrm{SD} /-$ Leu with glucose or sucrose as the carbon source and incubated for 2 days at $30^{\circ} \mathrm{C}$. Clones harboring functional signal peptides with the reconstituted invertase activity were able to grow on sucrose as the sole carbon source. Untransformed mutant yeast strain SEY 6210 and transformed SEY 6210 cells with empty 
pYST-0 vector were used as negative controls. Plasmid DNA was extracted from the positive clones and used to retransform E. coli. The constructs were again checked for the presence of signal peptide sequence by DNA sequencing (Eurofins, Louisville, KY).

\subsection{Tests for positive selection}

We focused our selection analysis on single-copy three-way orthologous groups with one or three predicted SP. We found 163 three-way SP-only orthologous groups, among which 150 were single-copy orthologous groups (i.e., single-copy three-way SP-only orthologous groups or single-copy core secretome). Furthermore, 118 single-copy orthologous groups retained a single predicted SP after annotation (i.e., single-copy threeway SP-mixed orthologous groups from the accessory-secretome, hereafter abbreviated as monoSP). As a first method to test for positive selection, we compared evolutionary codon models M8 and M8a (Yang et al., 2000) on 150 core and 118 monoSP single-copy orthologous groups using SELECTON (DoronFaigenboim et al. 2005). To check whether positive selection was more or less frequent in SPs compared to other (non-SP) genes, we performed the same test in 314 randomly picked single-copy three-way orthologous groups without predicted SP and with the same length distribution as predicted SPs. The evolutionary model $\mathrm{M} 8$, in which a proportion of sites are drawn from a category with $\mathrm{dN} / \mathrm{dS}$ ratio greater than one, i.e., allowing for sites undergoing positive selection, was tested against M8a, in which no site is allowed to have a dN/dS ratio larger than one, i.e., does not allow for positive selection, using a likelihood ratio test with one degree of freedom to determine the statistical probability that the genes evolve under positive selection (Stern et al. 2007). We adjusted chi-squared p-values using Bonferroni's correction for multiple testing in R considering 582 tests.

We also performed McDonald-Kreitman (MK) tests to infer the existence of positive selection (McDonald \& Kreitman 1991). MK tests contrast levels of polymorphism and divergence to test for a departure from neutrality in terms of non-synonymous substitutions (i.e., rapid amino-acid changes) while controlling for gene-specific mutation rates. MK tests estimate $\alpha$, the fraction of amino acid substitutions that were driven by positive selection. To analyze within-species polymorphism, we used genome sequences previously obtained with Illumina paired-end sequencing technology for populations of the three focal species M. lychnidis-dioicae, M. silenes-dioicae and M. violaceum var paradoxa (Whittle et al. 2015; 
Badouin et al. 2017; Branco et al. 2018). We downloaded raw data publicly available from the NCBI Short Read Archive (SRA) under the BioProject IDs PRJNA295022, PRJNA269361 and PRJEB16741. Four major genetic clusters were identified in Europe in M. lychnidis-dioicae (Badouin et al. 2017), and we only considered strains belonging to the largest cluster in North Western Europe so that population subdivision does not bias selection inferences. A list of the isolates used in the analysis is presented in supplemental table ST1. We processed the raw genome data of $18 \mathrm{M}$. silenes-dioicae, $20 \mathrm{M}$. lychnidis-dioicae, and four $M$. violaceum var paradoxa isolates to build pseudo-alignments sequences of gene coding sequences within each species using as reference genomes the assemblies reported in GCA_900015465.1 for M. lychnidis-dioicae, GCA_900120095.1 for M. silenes-dioicae and GCA_900015485.1 for M. violaceum var paradoxa. First, reads were trimmed for quality (length $>50$; quality base $>10$ ) using the Cutadapt v1.12 software (Martin 2011). We mapped Illumina reads against the reference genomes of each species using bowtie2 v2.1.0 (Langmead et al., 2009) and filtered for PCR duplicates using picard-tools (http://broadinstitute.github.io/picard). We realigned reads, called for SNPs and filtered them for quality, high genotyping rate $(>90 \%)$ and minor allele frequency $(>10 \%)$ using GATK version 3.7 (McKenna et al., 2010) and vcftools version 0.1.13 (Danecek et al., 2011) as described previously (Branco et al., 2018; Hartmann et al., 2018). We built pseudo-alignments sequences of gene coding sequences from the VCF file produced by GATK using a customized script. For each strain, reference nucleotides were replaced by their variants in the reference sequence. We used MUSCLE (Edgar, 2004) and translatorX (Abascal et al., 2010) to perform codon-based alignments of gene coding sequences among and between species. We used the MKT() and get.MKT() functions in the POPGENOME Rpackage (Pfeifer et al., 2014) to perform MK tests.

With these tools, we performed three comparisons. We tested for positive selection comparing polymorphism and divergence of 148 core secretome and 115 monoSP orthologous groups for (1) $M$. violaceum var paradoxa against $M$. lychnidis-dioicae and M. silenes-dioicae strains; (2) M. silenes-dioicae against $M$. violaceum var paradoxa strains; and (3) M. lychnidis-dioicae against $M$. violaceum var paradoxa strains. We excluded from the analyses genes having multiple (paralogous) copies. No neutrality index or $\alpha$ value could be computed for 27 orthologous groups in the pairwise species comparison (1), 67 orthologous groups in the pairwise species comparison (2) and 67 orthologous groups in the pairwise species comparison (3), due to lack of synonymous or non-synonymous polymorphism. We performed the same three pairwise 
comparisons with 314 genes from the control group described above. No neutrality index or $\alpha$ value could be computed for 30, 99 and 84 in the control pairwise comparisons (1), (2) and (3), respectively. We assessed significance of positive selection for genes having a neutrality index inferior to 1 and a positive $\alpha$ value using a Fisher test ( $\mathrm{p}$-value $<0.05)$.

\subsection{Footprints of RIP (repeat-induced point mutations)}

We investigated the extent of RIP-like footprints in Microbotryum genomes with a per-gene RIP-index defined as the ratio of $t$ over $n$ (RIP-index $=t / n$ ), with $t$ being the sum of TTG and CAA trinucleotides (forward and reverse potentially RIP-affected targets; Hood et al., 2005) divided by the sum TCG and CGA (forward and reverse non RIP-affected targets), and $n$ being the sum of all other non-target trinucleotides [ACG]TG and $\mathrm{CA}[\mathrm{CGT}]$ divided by the sum of $[\mathrm{ACG}] \mathrm{CG}$ and $\mathrm{CG}[\mathrm{CGT}]$, to control for contextual sequence composition. A RIP-index greater than one thus represents an excess of potentially RIPed sites controlling for the base composition. We compared the distribution of per-gene RIP-index values between genes predicted to encode SPs and those not predicted to encode SPs (non-SPs), and considering whether or not the genes belonged to orthologous groups undergoing positive selection.

\subsection{Genomic landscape analyses}

We used OcculterCut v1.1 (Testa et al., 2016) to determine if Microbotryum genomes harbored AT-rich regions. Contigs suspected to contain mitochondrial sequences were removed from the assemblies prior to the analysis using the mito_filter.sh script, available as part of the OcculterCut distribution (https://sourceforge.net/projects/occultercut). Transposable elements locations for M. lychnidis-dioicae and M. silenes-dioicae were retrieved from a previous study (Hartmann et al., 2018) and predicted in $M$. violaceum var paradoxa using the same TE centroid sequence database (Hartmann et al., 2018). Distance to TE was parsed with bedtools (Quinlan and Hall, 2010). 
2.7 Intraspecific secretome comparison between M. lychnidis-dioicae isolates from differentiated populations For analyzing the genome-wide intraspecific variation in secretomes, a second genome (assembly GCA_003121365.1) of M. lychnidis-dioicae isolated in Olomouc, Czech Republic, and abbreviated as M. lychnidis-dioicae 1318, was analyzed (Hartmann et al., 2018). We used blastp and orthAgogue to obtain high-scoring pairs between gene models of M. lychnidis-dioicae 1318 and the entire gene model set analyzed previously (Branco et al., 2018) and re-ran the mcl algorithm. We then parsed the extended orthologous groups to identify the M. lychnidis-dioicae 1318 gene models homologous to the M. lychnidis-dioicae Lamole SPs identified in this work. We compared the frequency of synonymous and non-synonymous single nucleotide substitutions in codon-based pairwise alignments of $M$. lychnidis-dioicae Lamole and $M$. lychnidis-dioicae 1318 genes corresponding to the core secretome or to the non-SP control single-copy orthologous groups. Per-site substitution numbers were calculated as the sum of substitutions divided by the length of the nucleotide alignment.

2.8 Analysis of gene expression level across infection stages and mating conditions

We retrieved gene expression data across M. lychnidis-dioicae Lamole infection stages on Silene latifolia and phytol-induced mating conditions from previous studies (Perlin et al., 2015; Toh et al., 2017; Toh et al., $2018)$ as average $\log 2$ fold change $(\log 2 \mathrm{FC})$ against the mated (non-infection) condition ( $\mathrm{n}=2-4$ for each of the eight conditions analyzed). We obtained the one-to-one gene model correspondences between long- and short-read sequencing-based assemblies of the same M. lychnidis-dioicae Lamole strain as best reciprocal hits with blastp. We focused our analysis on predicted SPs from the core and monoSP orthologs, using gene models from the control set described above for comparisons. Only genes with a Benjamini-Hochberg's adjusted p-value lower than $1 \mathrm{e}-5$ in at least one condition were considered. Clustering and plotting was performed in $\mathrm{R}$ with the heatmap. 2 function of the gplots package using 10 bins for colouring the $\log 2 \mathrm{FC}$ values and clustering by mean values per row. Pie charts were generated with the pie function of $\mathrm{R}$ base. 
2.9 Plotting, statistical tests, and figures

Unless otherwise stated all plots and statistical tests were performed in R version 3.6.1 (R Core Team, 2019).

Final layout of the figures was produced with Inkscape version 0.92.3.

\section{Results}

3.1 Overview of Microbotryum predicted secretomes

Analysis of the three Microbotryum secretomes revealed inventories of SPs of similar sizes in all three species. Initial prediction identified around 600 genes with signal peptides in each species (Figure C2-1). Utilizing sequence-based criteria of cellular localization and secretory signals, we kept 302, 371, and 418 SPs in M. violaceum var paradoxa, M. silenes-dioicae and M. lychnidis-dioicae, respectively, for further analysis. 
M. silenes-dioicae

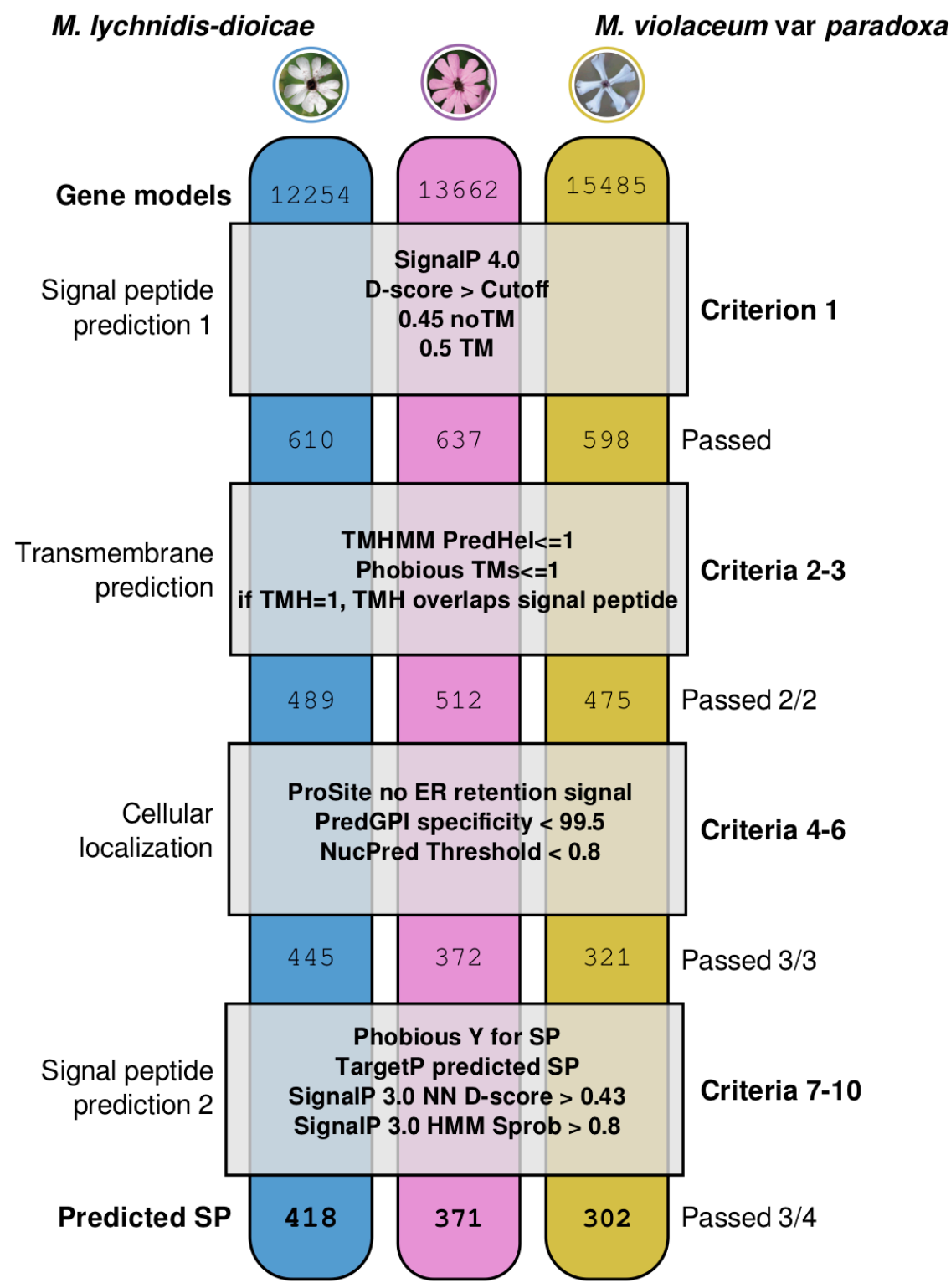

Figure C2-1 Procedural framework for predicting secreted proteins in three Microbotryum species.

The genomes for the three fungal species (M. lychnidis-dioicae, M. silenes-dioicae, and M. violaceum var paradoxa) were first screened to identify putative secreted proteins (criterion 1). The resulting proteins were then screened for transmembrane segments (criteria 2-3) and for conflicting cellular localization (criteria 46). Candidate secretory peptides were retained for further analysis if they passed all first six criteria (criteria 
1-6) plus at least three out of four additional signal peptide prediction cutoffs (criteria 7-10). Each column corresponds to a species, each box to the criteria employed and the numbers to the translated gene models that passed the criteria above. Image from Beckerson et al., 2019.

Over $85 \%$ of the predicted SPs were clustered into 453 orthologous groups, 225 comprising exclusively predicted SPs (645 SPs), henceforth called "SP-only", and 239 in which at least one member was not predicted as SP (298 SPs), henceforth called "SP-mixed" (Figure C2-1). Over two thirds of the predicted SPs belonged to orthologous groups with genes in all three species (753 predicted SPs in 163 SP-only and 177 SP-mixed groups). Further, 190 predicted SPs belonged to orthologous groups shared by only two species. Only 148 SPs (i.e., 14\% of the total) had no ortholog in two of the species and were therefore classified as species-specific SPs (62 in M. violaceum var paradoxa, 44 in M. lychnidis-dioicae and 42 in M. silenes-dioicae). Predicted SPs were significantly depleted in species-specific genes in all three species (Chisquare with Yates correction $\mathrm{p} \leq 0.0002)$. We classified as "core-secretome" $47 \%$ of the predicted SPs (513 genes belonging to 163 SP-only orthologous groups with members in all three species). In 118 SP-mixed orthologous groups with single-copy members in all three species, secretion signals were predicted in the orthologs of a single species, orthologs being non-SPs in the two other species; such orthologous groups will be referred to as "monoSP" hereafter (Figure C2-2 and Supplemental File SF1 from Beckerson et al., 2019). 


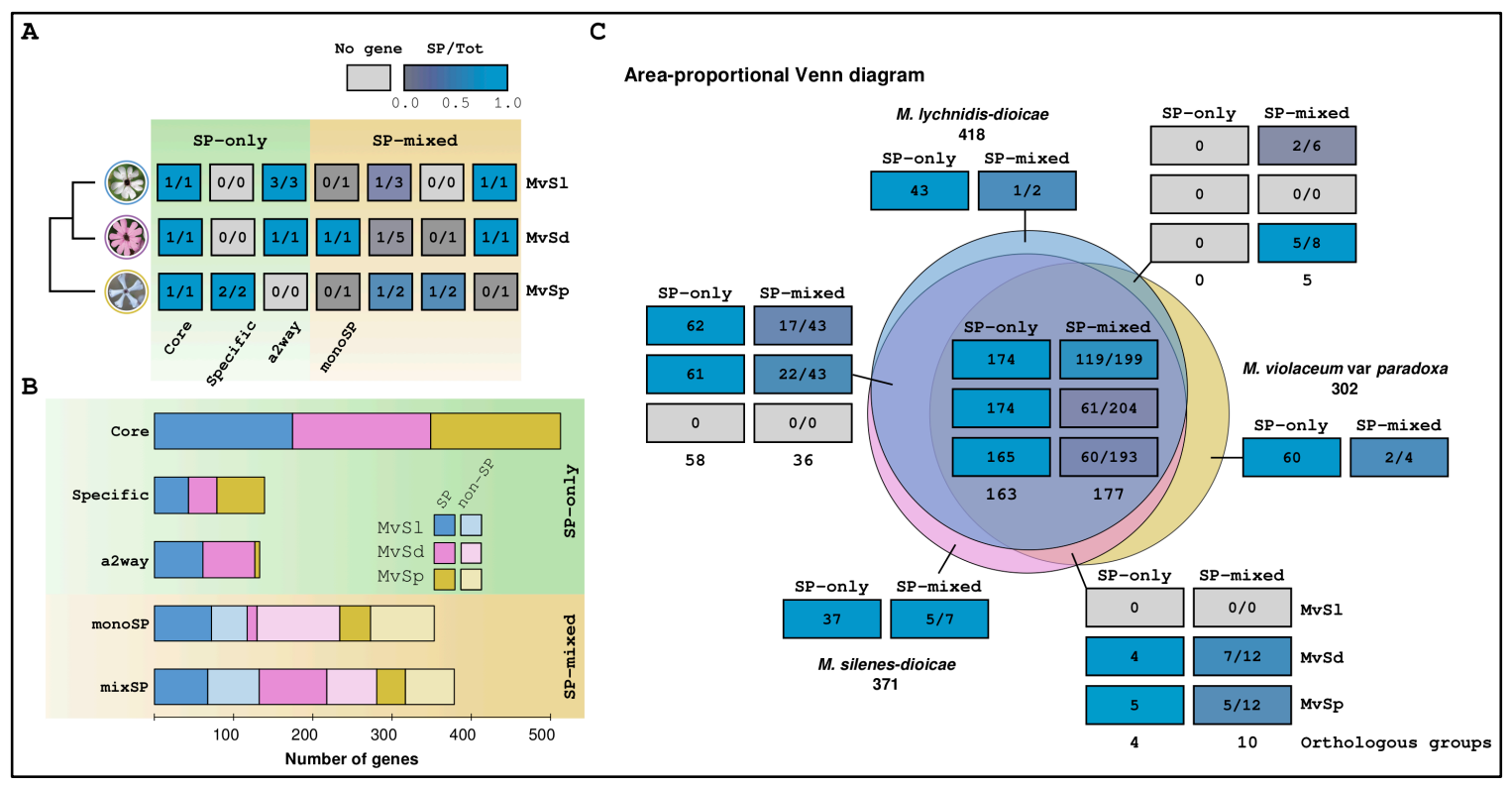

Figure C2-2 Comparison between the secretomes from three Microbotryum species. A) Key to the phylogenetic profile of predicted secreted protein (SP) and non-SP homologs with examples for the orthologous group terminology used in this study. Cladogram on the left shows the phylogenetic relationships of the three species. In the SP-only orthologous groups at left, with the light green background, all genes are predicted as secreted. In the core secretome, all three species have at least one predicted SP; in the speciesspecific orthologous groups, predicted SPs were represented in a single species (i.e., paralogous genes); in the accessory two-way (a2way) groups, one species did not have any ortholog in our reconstruction. In the SP-mixed orthologous groups at right, with the yellow background, not all orthologs were predicted as secreted; for example, in the monoSP group, a single species had predicted secreted proteins in the monocopy orthologous group. The box color key corresponds to the ratio of predicted SPs over the total number of genes in a given orthologous group per species, with a gradient from blue when all orthologs in all three species are predicted as secreted to dark gray when no ortholog is predicted as secreted. Pale gray boxes represent missing genes in a given orthologous group. B) Stacked bar plots of gene counts in the different categories described in the panel A, with the same terminology, light colors correspond to non-SP homologs of predicted SPs. C) Area-proportional Venn diagram of predicted SP and non-SP homologs, also including species-specific genes. Each area is annotated with six-cell blocks with the number and proportion of predicted SPs in SP-only and SP-mixed orthologous groups, respectively, colored following the same gradient as in panel A. Numbers at the bottom of the blocks correspond to the number of SP-only (left) or 
SP-mixed orthologous groups (right). Rows in the blocks correspond to M. lychnidis-dioicae, M. silenesdioicae, M. violaceum var paradoxa, from top to bottom. Venn diagram was obtained with BioVenn (Hulsen et al., 2008). Abbreviations for all panels: a2way, accessory SP two-way orthologous groups; Core, orthologous groups in which all members are predicted as SP and with at least one gene in each species; mixSP, orthologous groups with both SP and non-SP genes not including monoSP; monoSP, orthologous groups with one gene in each species but with a single predicted SP; MvSl, M. lychnidis-dioicae; MvSd, M. silenes-dioicae; MvSp M. violaceum var paradoxa; SP-mixed, orthologous groups with at least one gene not predicted as encoding a SP; SP-only, orthologous groups in which all genes are predicted as encoding SPs. Image from Beckerson et al., 2019.

The majority of SPs for each species were smaller than the median length of all predicted proteins in the three species (57\%, 68\% and 65\% of SPs were smaller than 361 amino acids for M. lychnidis-dioicae, M. silenes-dioicae, and M. violaceum var paradoxa, respectively; Figure 3a and Supplemental File SF1 from Beckerson et al., 2019). Initial screening of secretomes showed a high percentages of SPs without known Pfam domains, i.e., $52.1 \%$ in M. lychnidis-dioicae, $67.9 \%$ in M. silenes-dioicae, and $62.3 \%$ in M. violaceum var paradoxa. The percentage of genes without identified Pfam domains was even higher for predicted SPs smaller than 250 amino acids, i.e., $81.7 \%$ in M. lychnidis-dioicae, $88.9 \%$ in M. silenes-dioicae, and $84.0 \%$ in M. violaceum var paradoxa (Figure C2-3b). This trend was further observed when analyzing the subset of core SPs (Figure C2-3b and supplemental file SF1 from Beckerson et al., 2019). 


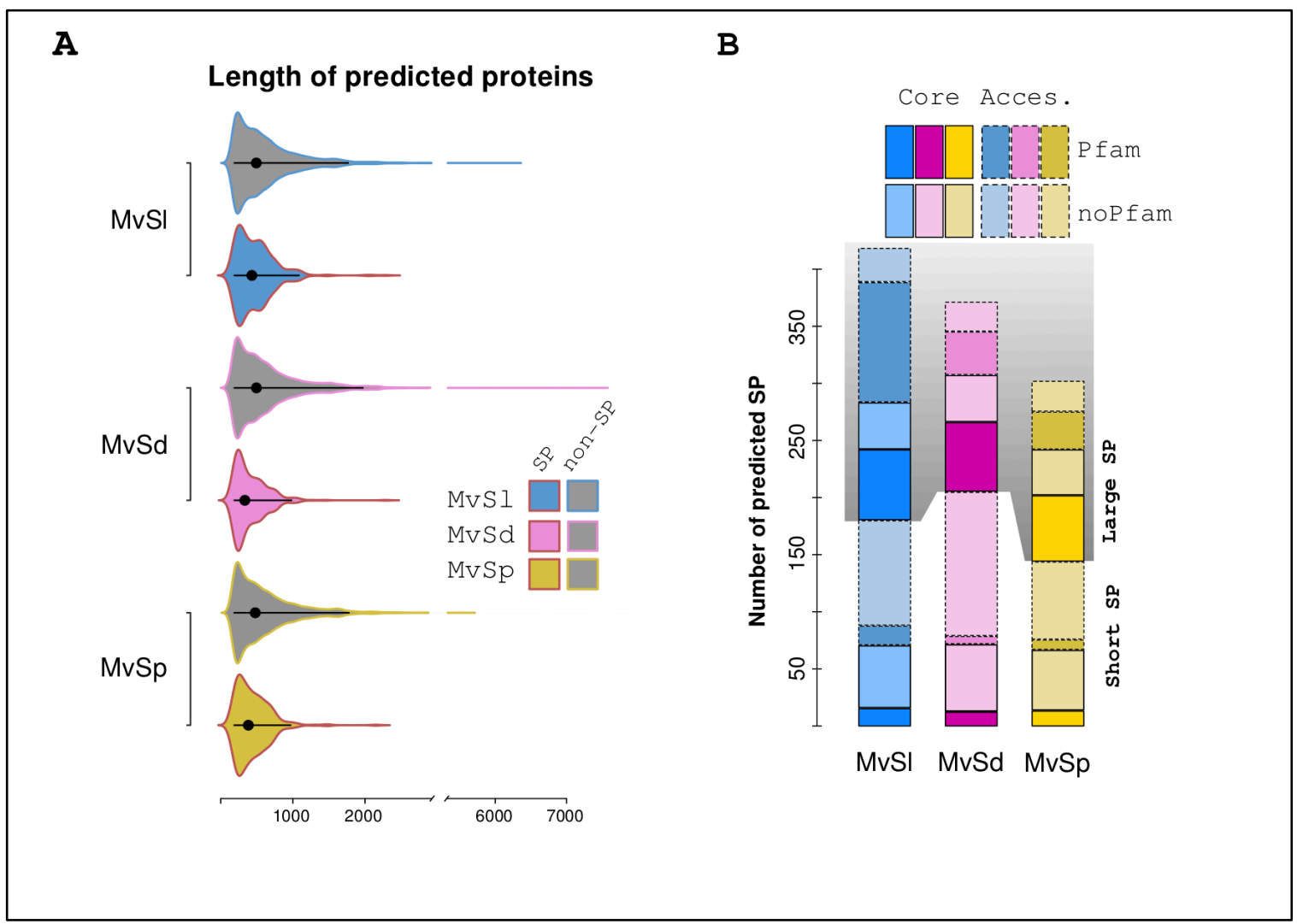

Figure C2-3 Overview of predicted SP (secreted protein) and non-SP homologs. A) Length distribution of predicted SPs (area colored by species) and non-SPs (gray area with outline colored by species) in the three species. Black bars and large black dots indicate the range containing $95 \%$ of the points and the median, respectively. B) Pfam screening results for predicted SP in each of the three species. Stacked bars show the number of predicted SPs with (dark colors) and without (light colors) hits among Pfam-A models. Predicted SPs from the core secretome are boxed with a continuous line and those from the accessory-secretome with broken lines. Shaded area corresponds to predicted SPs larger than 250 amino-acids (Large SP in the figure). Microbotryum species abbreviations are as in C2-2. Image from Beckerson et al., 2019.

\subsection{Signal peptide clusters and yeast secretion trap results}

The clustering of the signal peptides of predicted SPs resulted in 280 groups with two or more sequences at $75 \%$ sequence identity ( 823 sequences out of the 1091 predicted SPs). The signal peptides tested here together with the four previously tested (Kuppireddy et al., 2017) are representative of the signal peptides of 28 predicted SPs in the three Microbotryum species under study (Figure C2-4). To test whether the predicted 
secretion signals can indeed direct secretion, we used an invertase-deficient mutant of Saccharomyces cerevisiae. Such mutants can grow on glucose but not on sucrose unless transformed with a plasmid containing the invertase gene with a functional secretion signal, which allows the invertase to cleave extracellular sucrose into glucose and fructose in the medium. Cells of the invertase-deficient mutant SEY6120 of S. cerevisiae were transformed with pYST-0 vectors containing each tested signal peptide region upstream and in-frame with the invertase gene. As evidenced by the ability of their respective secretion signals to allow SEY6120 to grow on medium containing sucrose as the sole carbon source, all 9 predicted secreted proteins that have been tested so far using yeast secretion trap have been confirmed to be secreted (Figure C2-4 and Kuppireddy et al., 2017). Interestingly, protein 12964 from M. violaceum var paradoxa, was originally filtered out of our list of predicted SPs, due to the prediction that it is GPI-anchored to the membrane. Nevertheless, in this assay using only the secretion signal of the protein, invertase was secreted, suggesting that our conservative approach to estimating secretion may initially filter out membrane proteins with potential functional components outside the fungal cell.

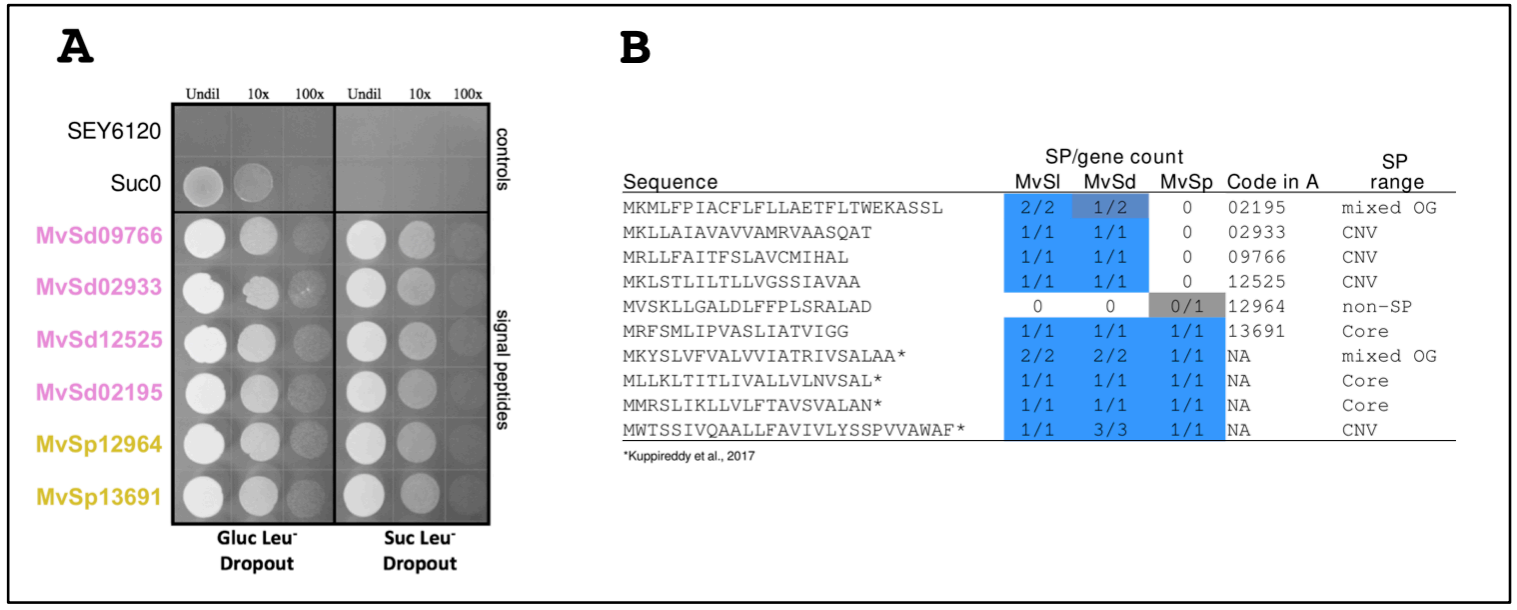

Figure C2-4 Experimental validation of predicted signal peptides. A) Yeast secretion trap analysis of a subset of putative secreted proteins from Microbotryum silenes-dioicae and M. violaceum var paradoxa. The invertase deficient mutant SEY6120 of Saccharomyces cerevisiae is shown in the top row and represents a negative control on medium containing sucrose as the sole carbon source. SEY6120 cells transformed with the pYST-0 vector without a signal peptide upstream of the invertase gene is shown in the second row. Such cells are able to grow on the glucose -leu dropout medium, but not when sucrose is the sole carbon source. 
The SEY6120 cells in the following six rows are transformed with a construct in which the signal peptide region corresponding to the putative secreted protein ID listed on the left of the row is fused to the truncated $S U C 2$ gene. If the signal peptide allows secretion, then the transformed $S$. cerevisiae cells are able to grow on sucrose as the sole carbon source. Different dilutions of cells were made (undiluted, diluted 10x or 100x) to better distinguish differences, if any. B) Amino acid sequences and species range of signal peptides tested here and in a previous study (Kuppireddy et al., 2017). Cells under the "SP/gene count" columns follow the same color scheme as in Figure C2-2. Microbotryum species abbreviations are as in Figure C2-2. The signal peptide with the code 12964 in panel A corresponds to a protein from M. violaceum var paradoxa predicted to be GPI-anchored to the membrane. Image from Beckerson et al., 2019.

\subsection{Interspecies comparison of Microbotryum predicted secretomes}

As expected, due to their phylogenetic placement, the orthologous proteins of $M$. silenes-dioicae and $M$. lychnidis-dioicae were more similar (median identity 98.7\%) than either of the two sister groups compared to M. violaceum var paradoxa (median $86.9 \%$ for M. lychnidis-dioicae / M. violaceum var paradoxa and 87.1\% for M. silenes-dioicae / M. violaceum var paradoxa). Orthologous SPs, including those belonging to the core secretome, were significantly less similar to one another than control non-SPs from single-copy orthologous groups of similar lengths (Wilcoxon rank sum test with continuity correction $p<7 e-7$ for all three pairwise between-species comparisons, Figure C2-5). Out of the 150 single-copy orthologous groups with a SP predicted in each of the three species, i.e. most of what we call the core secretome (leaving out 13 single-copy orthologous groups with more than one gene in at least one species), we identified 92 groups with codons exhibiting more non-synonymous substitutions than synonymous substitutions. Likelihood ratio tests comparing models with or without positive selection indicated that the model with positive selection was significantly more likely in 18 of these groups (Bonferroni multiple test-corrected p-value $<0.05$, supplemental file SF2 from Beckerson et al., 2019). Similarly, we identified 74 out of 118 monoSP orthologous groups with codons exhibiting $\mathrm{dN} / \mathrm{dS}$ values above one, among which multiple test-corrected likelihood ratio tests revealed 21 orthologous groups evolving under positive selection. Selection tests on the 314 control orthologous groups of similar lengths as SPs returned 20 groups evolving under positive selection. Core secretome and monoSP orthologous groups were found enriched in proteins with signs of 
positive selection (Fisher's exact text $\mathrm{p}=0.02505$ for core versus control and $\mathrm{p}<0.00048$ for monoSP versus control; supplemental files SF1 and SF2 from Beckerson et al., 2019). We found nine core and fourteen monoSP orthologous groups under positive selection with hits in the Pfam-A database (supplemental file SF1 from Beckerson et al., 2019), among which pectinesterase (PF01095.19) and chitin deacetlyase (PF01522.21) have been implicated in fungal biotrophy, potentially for the manipulation of host development (Juge, 2006; Perlin et al., 2015). Glycosyl hydrolases (GHs) (PF00295.17 and PF00704.28) were found in the core and monoSP orthologous groups, despite an overall paucity of GHs represented among M. lychnidis-dioicae genes (Perlin et al., 2015). Enzymes of these particular families are interesting due to their ability to hydrolyze pectin, a process important in both pathogenic and saprophytic fungi life stages (Sprockett et al., 2011).

\subsection{Intraspecific comparisons of Microbotryum predicted secretomes}

We further investigated footprints of positive selection using McDonald-Kreitman (MK) tests that compare the amount of variation within a species (polymorphism) to the divergence between species (substitutions) at two types of sites, synonymous and non-synonymous. A ratio of nonsynonymous to synonymous polymorphism within species lower than the ratio of nonsynonymous to synonymous differences between species indicates positive selection (McDonald \& Kreitman 1991). We performed three pairwise species comparisons between M. violaceum var paradoxa, M. lychnidis-dioicae and M. silenes-dioicae, using 148 core, 115 monoSP and 314 control orthologous groups. We used population genomics data from 20, 18, and 4 isolates from M. lychnidis-dioicae, M. silenes-dioicae, and M. violaceum var paradoxa, respectively (Whittle et al. 2015; Badouin et al. 2017; Branco et al., 2018; supplemental table ST1 from Beckerson et al., 2019). Figure C2-5A shows the locations where the isolates were sampled. The MK tests indicated signatures of within-species positive selection in eight core secretome orthologous groups and fifteen monoSP orthologous groups (supplemental file SF3 from Beckerson et al., 2019). Out of the 23 orthologous groups with signatures of positive selection detected using MK tests, six were also detected to evolve under positive selection in the SELECTON analysis (supplemental file SF1 from Beckerson et al., 2019). Five orthologous groups were found undergoing intraspecific positive selection in all three comparisons. Intraspecific selection tests on control non-SP orthologous groups revealed that 11 underwent positive selection. While core SPs showed no excess of fixed non-synonymous polymorphisms, monoSPs were enriched in genes evolving 
under within-species positive selection (15 out of 115 monoSPs versus 11 out of 314 non-SP genes, Fisher's exact test $\mathrm{p}=0.0008147)$.

When we compared two well-assembled M. lychnidis-dioicae genomes, those of the Lamole and 1318 strains, originating from two differentiated populations maladapted to their sympatric hosts (Feurtey et al., 2016), we only found 29 Lamole M. lychnidis-dioicae SPs without a corresponding 1318 M. lychnidisdioicae gene (12 predicted SPs in 10 orthologous groups and 17 species/strain-specific SPs). In addition, we found 11 orthologous groups for which gene model counts were different between the 1318 and Lamole $M$. lychnidis-dioicae strains. The ratio of SP-containing orthologous groups with gene count polymorphisms between M. lychnidis-dioicae strains was significantly smaller than the genome-wide ratio (21/357 SPs vs 2642/12277 all genes, Chi-square with Yates correction $\mathrm{p}<1 \mathrm{e}-11$ ). We found few predicted SPs within genome regions showing presence/absence polymorphism within species as analyzed previously (Hartmann et al., 2018) in both M. lychnidis-dioicae Lamole (five) and M. silenes-dioicae (two). Substitutions, on the other hand, were more frequent between M. lychnidis-dioicae Lamole and M. lychnidis-dioicae 1318 strains in predicted SPs than in control genes (Wilcox rank sum test with continuity correction $p=2.537 \mathrm{e}-05$, Figure C2-5c and supplemental file SF4 from Beckerson et al., 2019). 


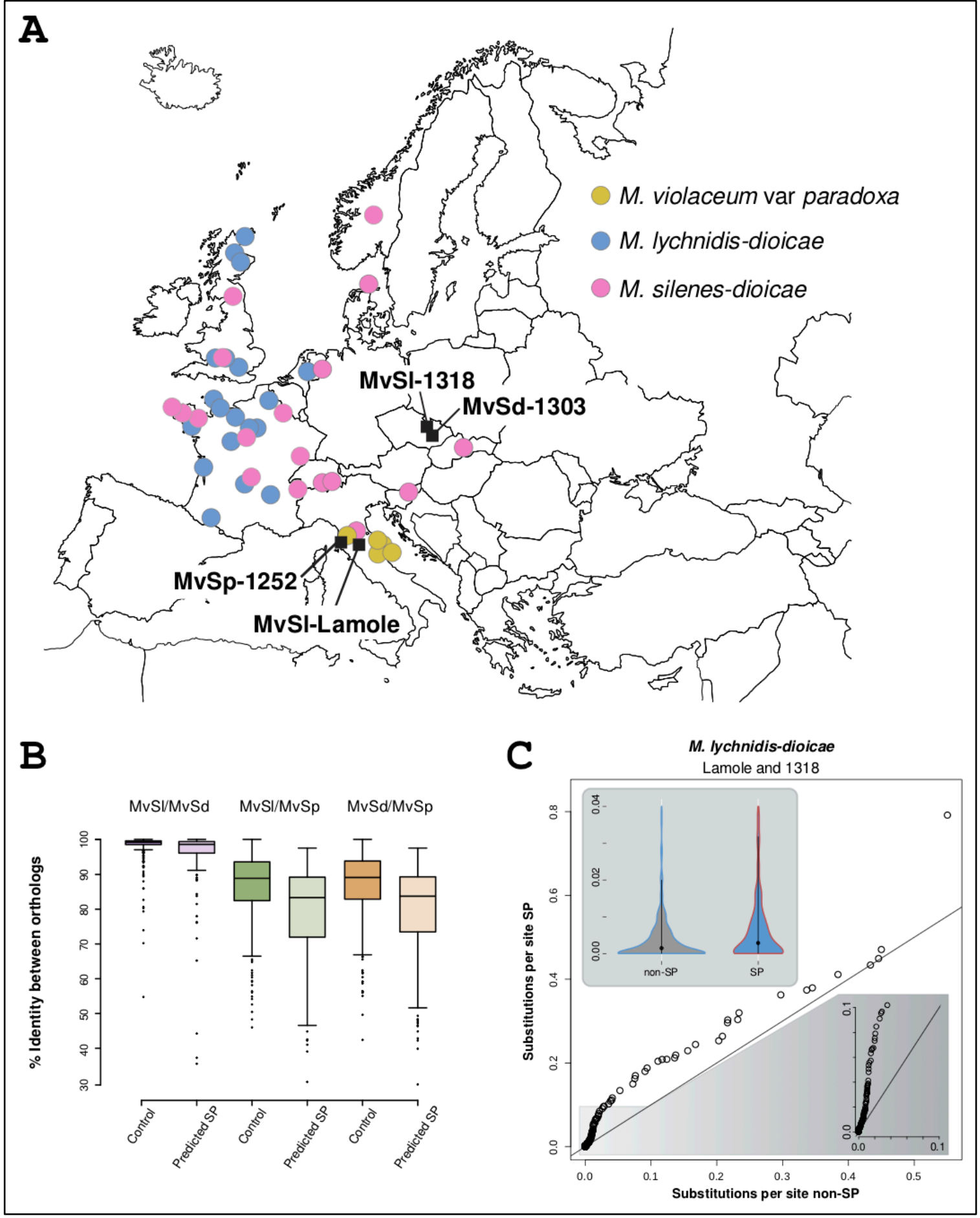

Figure C2-5 Inter- and intra-specific comparisons of Microbotryum secretomes. A) Sampling locations of the isolates used in this study. B) Distribution of pairwise percentage of amino-acid sequence identity between predicted SPs and background orthologous genes from M. lychnidis-dioicae, M. silenes-dioicae and M. violaceum var paradoxa. C) Quantile-quantile (main) and violin (inset) plots of substitution numbers per 
site between two strains of M. lychnidis-dioicae from Lamole, Italy (MvSl-Lamole), and from Olomouc, Czech Republic (MvSl-1318). The shaded area at the bottom right zooms into the low divergence zone of the quantile-quantile plot. The straight lines correspond to a 45 degree reference line (i.e., points would fall close to this line if the two data sets have the same distribution). Microbotryum species abbreviations in A and B are as in Figure C2-2.

\subsection{Genomic context of predicted SPs}

In contrast to some other plant pathogenic fungi with effectors frequently located in repeat-rich regions, we did not find genes encoding predicted SPs to be significantly closer to transposable elements than other genes (Figure C2-6) and found no evidence for genome compartmentalization into AT-rich or GC-rich regions in any of the three genomes analyzed, extending previous observations (Perlin et al., 2015). We nevertheless estimated the frequency of sites potentially affected by the RIP-like mechanism reported in Microbotryum fungi, targeting TTG and CAA trinucleotides. We calculated a RIP index that takes values above one when there is an excess of TTG and CAA trinucleotides over the corresponding target sites not affected by RIP (TCG and CGA), controlling for local sequence composition (see Methods). The coding regions of predicted SPs did not show any significant excess of RIP-affected trinucleotides, regardless of whether the orthologous groups showed signs of positive selection (Figure C2-6). Our RIP-index measure was negatively correlated with distance to transposable elements (TEs), indicating RIP leakage to TE-neighboring regions. The RIP index was not correlated with the ratio between non-synonymous and synonymous substitutions (Figure C26), indicating that the RIP-like mechanism does not play a significant role in the diversification of genes under positive selection in Microbotryum fungi. 


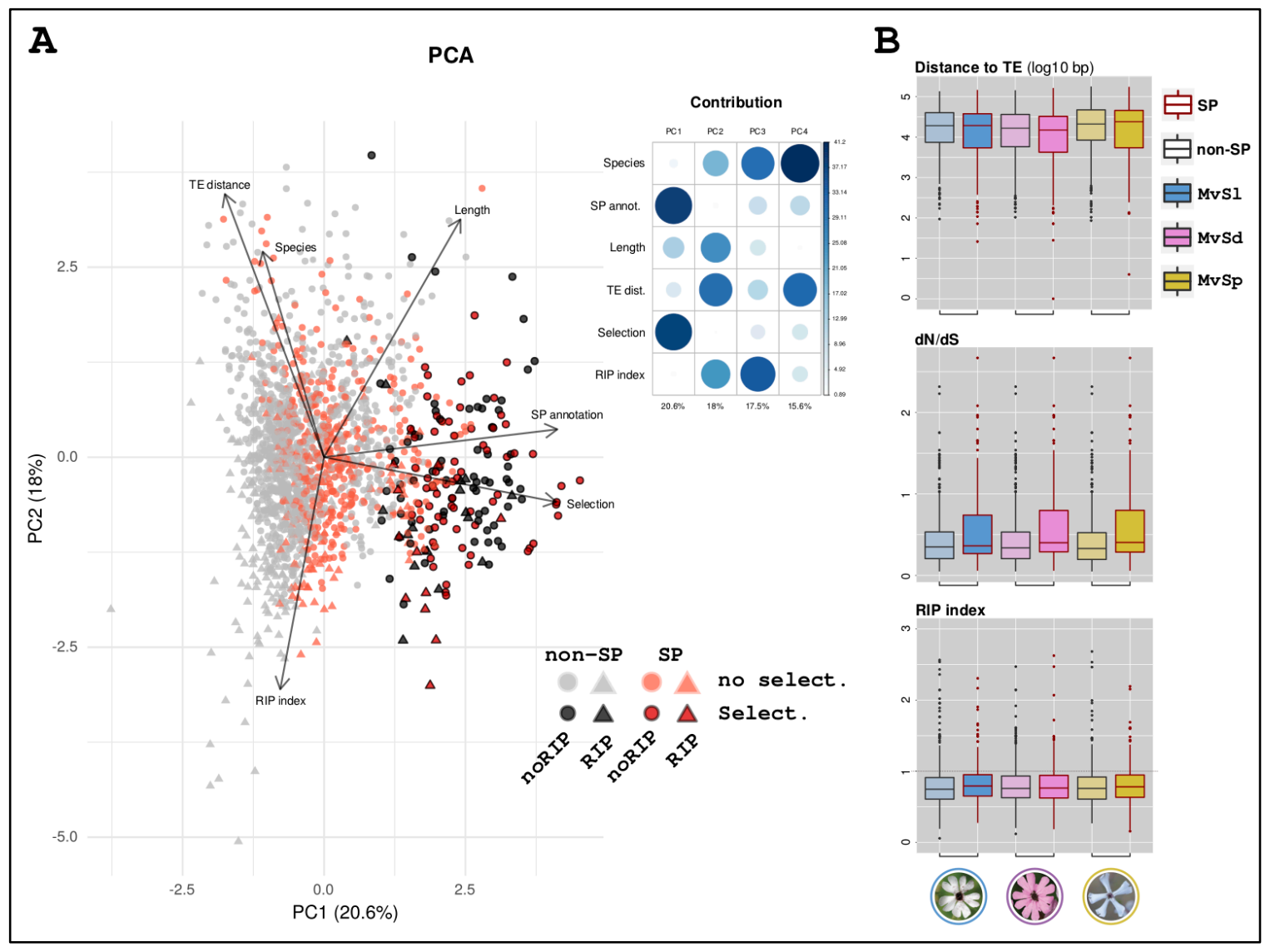

Figure C2-6 Investigation of the impact of RIP (repeat-induced point mutations) on gene

diversification among species. A) Principal component analysis (PCA) of gene copies according to their trait value for six variables : (i) their annotation as binary variable, i.e. encoding secreted protein SP (genes colored in red) or non-SP (in grey), (ii) their length in bp as continuous variable, (iii) the species they belong to as category variable (MvSl: Microbotryum lychnidis-dioicae, MvSd: M. silenes-dioicae, MvSp: M. violaceum var paradoxa), (iv) their distance to the nearest transposable element as continuous variable (TE distance), (v) their RIP index as continuous variable (RIP-affected gene noted as triangles and non RIPaffected genes as circles) and (vi) the detection of positive selection (genes with dark colors) or the lack of positive selection (light colors) as binary variable. The projection of the variables is plotted as arrows in the space defined by the first (PC1) and second (PC2) components and the percentage of the total variance explained by each principal component is provided in brackets. The arrows representing the variable projection were scaled for better visualization (6-fold magnification). The contribution of the variables to principal components is shown in a correlation plot (upper right). B) TE distance, $\mathrm{dN} / \mathrm{dS}$ (synonymous substitutions over non-synonymous substitutions) and RIP index distribution of predicted SPs (red contour) 
or non-SPs (grey contour) in the three species (area colored according to species). Distance to TE was transformed as $\log 10 \mathrm{bp}$ distance; $\mathrm{dN} / \mathrm{dS}$ was calculated within orthologous groups. The boxplots represent the median (center line), the 25th percentile and 75th percentiles (box bounds), 1.5 times the distance between the 25 th and the 75 th percentiles (whiskers), and points being the outliers. Image from Beckerson et al., 2019

\subsection{Expression of predicted SPs across infection stages}

We focused our analysis on M. lychnidis-dioicae Lamole genes expressed in at least one of the five infection stages or three mating conditions for which we retrieved expression data (Perlin et al., 2015; Toh et al., 2017; Toh et al., 2018). Among the 2,840 genes fulfilling this condition, we found 135 and 58 predicted SPs from the single-copy core and monoSP orthologous groups, respectively, and compared their expression profiles to 232 genes from the non-SP control group (same length distribution but not predicted as potential effectors). Hierarchical clustering of expression profiles across infection stages grouped the genes into low (31 genes, median log2FC range $-7.35-4.15)$, medium (117 genes, median log2FC range $0.0-1.8)$, high (29 genes, median log2FC range 9.19 - 12.40), and no change (248 genes, median log2FC 0) average gene expression across infection stages. We found no major changes in expression of core, monoSP or non-SP genes across three mating conditions. Predicted SPs from the core orthologous groups were enriched among genes with high or low average expression across infection stages, respectively 19 and 18 out of 135 core SPs compared with 7 and 6 out of 232 control genes (Fisher's two tailed exact test $p=1.8 \mathrm{E}-3$ and 1.1E-3, respectively; Figure C2-7). In line with the pattern observed across all predicted SPs, we could infer the function of only 14 core and 7 monoSP genes with either high or low average expression. Glycosyl hydrolases, often involved in pathogenesis (Sprockett et al., 2011), were among the most common hits (supplemental files SF1 and SF5 from Beckerson et al., 2019). 


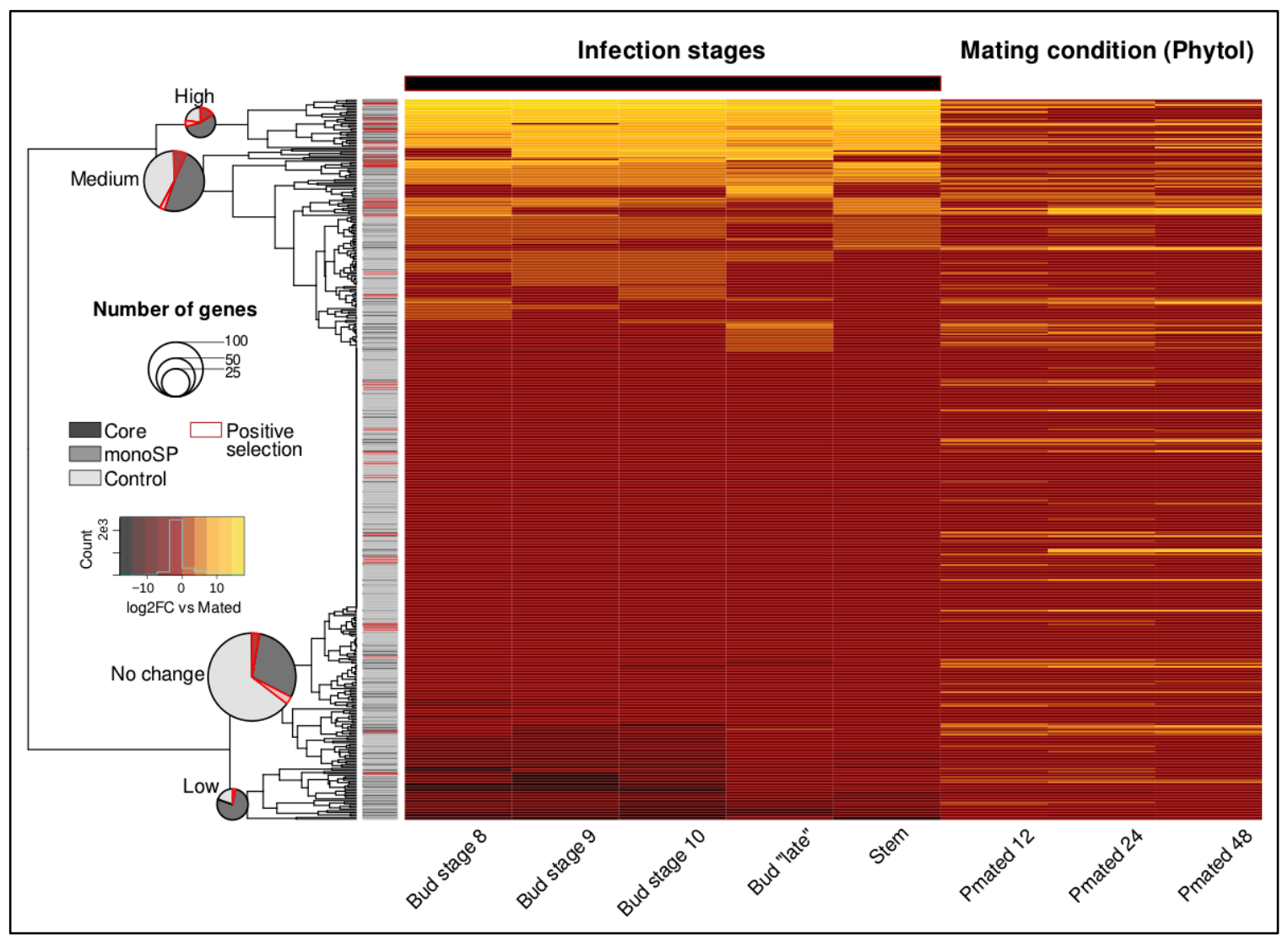

Figure C2-7 Relative expression of Microbotryum lychnidis-dioicae genes across infection stages on

flower structures. Heatmap of average gene expression $(n=2-4)$ across infection stages in flower structures (Toh et al., 2018) and mating conditions (Toh et al., 2017) as $\log 2$ fold change against a non-infection condition (mating on Phytol, "Pmated"). Hierarchical clustering based on mean row values across the infection stages (horizontal black bar) distinguish four expression profiles with average log2 fold change median values as follows: low, -6; no-change, 0 ; medium, 1.36; high, 12. Sidebar represents the annotation of the genes following the color scheme on the left. Pie charts detail the proportion of SP (core and monoSP) and non-SP (control) genes in each expression profile cluster. Pie chart area is proportional to the number of genes in each expression profile cluster. Red shades and outlines indicate genes with signatures of positive selection. Image from Beckerson et al., 2019. 


\section{Discussion}

Microbotryum secretomes appeared as largely shared among species, i.e., with few gene gains/losses. Instead, we found SPs to be rapidly evolving as these were more differentiated among species and more often under positive selection compared to non-SP genes, indicating that many SPs likely evolved under diversifying selection among species parasitizing different hosts. Such rapid evolution was also indicated by the low percentage of SPs matching Pfam domains (31-47\%), a percentage that decreased to less than $20 \%$ for the small secreted proteins. Such a finding regarding the lack of identifiable Pfam domains of a substantial proportion of SPs is consistent with previous reports in other smut pathogens and is a hallmark of secreted effectors involved in host-specificity (Jones et al., 2018). Diversifying selection in Microbotryum SPs is likely due to coevolution within species, local adaptation or specialization to different hosts, involving rapid changes in the sequences of secreted proteins to avoid detection in the plant and, more generally, to counteract evolving host defenses. Such a hypothesis is reinforced by the finding that SPs under positive selection were more often highly expressed in planta than non-SP genes. Although we found few species-specific SPs or with copy-number variation, these accessory SPs may also be involved in coevolution, local adaptation, and/or host specialization (Plissonneau et al., 2018; Schuster et al., 2018).

The results from the intraspecific comparison between the two M. lychnidis-dioicae strains shed further light on coevolution and local adaptation. We indeed found SPs to be more differentiated than nonSPs between two strains from genetically differentiated populations. These findings further support the idea that coevolutionary pressures may be causing divergence in effectors between differentiated populations of pathogens. In fact, the populations from South and Eastern Europe were genetically differentiated in both $M$. lychnidis-dioicae and its host plant Silene latifolia, and the plant showed local adaptation to the fungus (Feurtey et al., 2016), indicating the occurrence of coevolution. Gene presence-absence polymorphisms in M. lychnidis-dioicae, corresponding to the pathogen and host phylogeographic structure (Hartmann et al., 2018), and numerous selective sweeps across the genome (Badouin et al. 2017), further supported the existence of coevolution. In contrast with several crop pathogens (e.g., Plissonneau et al., 2016; Hartmann and Croll, 2017), neither presence-absence polymorphisms nor selective sweep regions were enriched in predicted SPs, even though nearly $10 \%$ of SPs were found located within recent selective sweeps in $M$. lychnidis-dioicae, which suggests recent adaptive events involving some SPs. 
The identification of a set of shared and conserved SPs, i.e., the 126 core-secretome orthologous groups without positive selection, was also interesting, providing a starting point to search for effectors that play a central role in the common pathogenicity traits of these fungi, e.g., the effectors that allow the fungi to migrate to the plant anthers, to induce stunted ovary and pseudoanther development in female flowers, and to eliminate and replace host pollen with fungal spores. The observed differential expression of core secreted proteins further narrows the search for these central effectors and points to sets of genes within the secretome that may play other central roles in the fungal life cycle, including the secretion of extracellular enzymes for carbon source metabolism. Indeed, phosphatases, peptidases, lipases and glycosidases accounted for half of the Pfam annotations of core-secretome orthologous groups with no signs of positive selection (20 out of 38 ). While such enzymes are clearly associated with fungal pathogens (Brown et al., 2015; Monod et al., 2002; Keyhani, 2018), they are often found in animal (Monod et al., 2002; Keyhani, 2018), and necrotrophic plant pathogens (Sprockett et al., 2011; Reis et al., 2005; Gacura et al., 2016), rather than in biotrophic fungi. On the other hand, the up-regulation of many carbohydrate active enzyme genes related to cell wall degradation was also seen in both wheat stem and poplar rust, P. graminis and $M$. larici-populina, respectively (Duplessis et al., 2011). In the case of M. lychnidis-dioicae, GH28 polygalacturonase domain-containing proteins were up-regulated during infection and were among the proteins with signs of positive selection enriched in the core secretome and monoSP orthologous groups. Since polygalacturonase is required for the pathway implicated in pollen dehiscence (Wang et al., 2016), this is consistent with a fundamental role for such enzymes in the pathogenic lifestyle of anther-smut fungi.

Future research with Microbotryum will utilize these findings to better understand the function of the most promising SP candidates, by identifying their targets within each host. Such research geared towards identifying the targets of secreted effectors from M. lychnidis-dioicae in its corresponding host plant, Silene latifolia, has already made progress (Kuppireddy et al., 2017). For instance, we identified here MvSl-1064A1-R4_MC02g04003 as part of the core secretome undergoing diversifying selection across species. We also found its transcript among the most highly expressed across infection stages. Its predicted protein product (residues 21-156) has been shown to interact with two host proteins in yeast two-hybrid assays (Kuppireddy et al., 2017). Extension of such work to analyze candidate effectors herein identified through in silico studies should add new insights into their relevance in host preference and the evolution of the Microbotryum species 
complex. By narrowing down the genomes and identifying prime candidates that are likely to play a major role in the pathogen's life cycle, this work helps to bridge the gap between the quickly expanding availability of Microbotryum genomes (Branco et al., 2017, 2018; Hartmann et al., 2019) and the emerging cellular and molecular biology work being done to understand the role of effectors in this system (Kuppireddy et al., 2017).

More generally, this study showed that the molecular changes that lead to different host ranges between closely related plant pathogens, or different locally-adapted genetic clusters, involved little gene gains/losses in their secretome but instead rapid evolution of shared secreted proteins. This represents a significant advance in our understanding of pathogen evolution and may contribute to understanding host shifts and emergent diseases. 


\section{CHAPTER 3}

\section{FUNCTIONAL CHARACTERIZATION OF THE CONSERVED BUT DIFFERENTIALLY ANNOTATED EFFECTOR, MVLG_02245}

\section{Introduction}

As the capacity to perform genetic sequencing continues to gain more widespread accessibility, genomic comparisons between closely related organisms has become the standard for delineation of species, especially concerning asexual microorganisms. Robust genome testing is in fact used for rapid identification of clinically relevant microbial pathogens and is capable of both detecting specific strains of microbes as well as characterizing new species (Hasman et al., 2014). The same can be said about using genome sequencing combined with bioinformatic tools to evaluate the coevolutionary trajectories between these pathogens and their various hosts.

Such is the case for the Microbotryum genus of anther-smut fungi, for which nearly 20 genomes have been sequenced in the genus over the past few years (Hartmann et al., 2019), and bioinformatic comparisons between species have identified conserved and species-specific effectors amongst the group (Beckerson et al., 2019). While these predictive approaches can help identify rapid evolution of effectors that play a vital role in pathogen/host coevolution, recent studies have demonstrated that bioinformatics analyses, while effective for identification of candidate genes, are not alone sufficient for the identification of every predicted protein function (Pevsner, 2015; Eisenhaber, 2013; Droite, Poirier, and Hunter, 2005;), especially for small secreted proteins which lack Pfam domains and GO terms. Furthermore, while bioinformatic tools are essential for narrowing down the proteome to a list of candidate effectors, they are unable to predict the target molecules within the host for secreted proteins. Thus, molecular genetic analysis of effectors remains an important step in describing host/pathogen relationships.

Like many other fungal pathogens, Microbotryum fungi utilize an array of effectors to manipulate their Caryophyllaceae plant hosts; however, despite the rich scientific history and abundance of genomic data 
available for this model system, only a handful of genes have characterized using molecular tools (Kuppireddy et al., 2017). In order to infect their hosts, Microbotryum possess an inventory of effectors to repress plant defense responses in order to infiltrate and reproduce inside their hosts, as well as to manipulate the host during their migration to the anthers and eventual replacement of the host's pollen with their own fungal spores (Schäfer, 2010). Based on this limited body of preliminary research, the Microbotryum/Caryophyllaceae complex does not exhibit a gene-for-gene relationship as seen in other species of phytopathogenic fungi, e.g., rusts (Liu, 2017; Thrall, 2016), which begs the question: what is the role of conserved small secreted proteins within the Microbotryum genus? While our previous studies have identified a handful of species-specific genes within the Microbotryum genus, bioinformatic comparisons of their secretomes has indicated that host-specialization in the genus is likely due instead to rapidly evolving shared sets of effectors (Beckerson et al., 2019). Of the secreted effectors identified by Beckerson et al., many had orthologous variants predicted to be non-secreted in other species (Beckerson et al., 2019). Therefore, host-specialization in the Microbotryum complex may be driven not only by stepwise changes to core-SPs, but also by the mobilization of effectors through changes to the signal peptide region of the gene.

In this research project, we analyze the molecular function of one such potential "mobilized" SP, MVLG_02245, a particularly conserved candidate SP with orthologs across the Microbotryum genus, but whose annotation is predicted differently between the first Microbotryum genome through JGI (https://mycocosm.jgi.doe.gov/Micld1/Micld1.home.html), and more recently sequenced genomes utilizing PacBio technology. Although more recent PacBio generated assemblies and annotation methods have not categorized MVLG_02245 as a protein, gene expression data suggest that MVLG_02245 is expressed, at least at the transcriptional level, in M. lychnidis-dioicae, and upregulated during infection. The changes observed in the signal peptide region of the conserved MVLG_02245 gene across species and the differences in its annotation between previous and more recent genome publications makes this particular putative effector an interesting candidate for both its functional analysis and its evaluation as an interesting case study for the difficulty of describing rapidly evolving effectors using bioinformatics alone. 


\section{Materials and Methods}

2.1 Bioinformatic analysis of MVLG_02245 in Microbotryum

MVLG_02245 was initially predicted to be secreted by the annotation provided through the BROAD Institute (Perlin et al., 2015; currently maintained and updated at JGI); however, upon comparison to the Pacific Bioscience (PacBio) genome for M. lychnidis-dioicae (GCA_900015465.1) for M. lychnidis-dioicae Lamole, it was concluded that while the DNA coding sequence was found in both genomes the latter method did not predict it to be a protein.

Bioinformatic comparisons were carried out using the MVLG_02245 sequence of M. lychnidisdioicae, retrieved from JGI (https://mycocosm.jgi.doe.gov/Micld1/Micld1.home.html) for BLAST analyses against the PacBio genome sequence of the same strain (GCA_900015465.1) M. lychnidis-dioicae Lamole, and the PacBio genomes QPIF00000000 M. silenes-dioicae 1303 a2 from Silene dioica and GCA_900015495.1 M. violaceum var. paradoxa from Silene paradoxa 1252 a1. Blastn and Blastp were performed using the NCBI local alignment tool (ncbi-blast-2.7.1+-x64-linux.tar.gz) Pfam 32 and HMMER 3.1b1 suite (https://hmmer.org) were used to screen the JGI translated gene model MVLG_02245 for any known protein families using a cutoff value of 1e-3 for significance. The coding DNA and translated protein sequences were also blasted against the NCBI online database to identify any similar proteins. SignalP4.1 was used to predict the secretion of coding sequences obtained from JGI, as well as to determine the signal peptide and functional protein regions of the corresponding translated protein sequence. PONDR and IUPred2A were used to screen for ordered protein folding of the protein sequence. Expression data were obtained from (Perlin et al., 2015; Toh et al., 2018) to verify production of the MVLG_02245 transcript.

\subsection{Yeast Secretion Trap of MVLG_02245}

SignalP4.1 was used to predict the signal peptide region of the translated protein for use in the Yeast Secretion Trap (YST) verification of protein secretion signals (Figure 1). Signal peptide regions that code for secretion of the following polypeptide can be used with the YST Suc2 plasmids, in combination with mutant yeast strains, to allow for secretion of an invertase protein capable of breaking down sucrose into its glucose and fructose monomers. Since these mutant yeast strains are not able to transport sucrose into the cell, secreting the invertase enzyme allows for growth of the strain on media where sucrose is the sole carbon source. The 
signal peptide region for MVLG_02245 was cloned in-frame and upstream of the plasmid-encoded Suc2 invertase gene. Standard PCR was used to amplify the signal peptide region using an initial denaturation phase at $96{ }^{\circ} \mathrm{C}$ for $5 \mathrm{~min}$, followed by 35 cycles of: 1) denaturation at $96{ }^{\circ} \mathrm{C}$ for $30 \mathrm{sec}, 2$ ) annealing at $60{ }^{\circ} \mathrm{C}$ for $30 \mathrm{sec}$, and 3) elongation at $72{ }^{\circ} \mathrm{C}$ for $30 \mathrm{sec}$. The program concludes with one final extension period of 5 min at $72{ }^{\circ} \mathrm{C}$ before maintaining a $4{ }^{\circ} \mathrm{C}$ temperature indefinitely. The resulting amplified signal peptide coding sequence was purified from agarose gel after gel electrophoresis and subcloned into pCR ${ }^{\mathrm{TM}} 2.1$ $\mathrm{TOPO}^{\mathrm{TM}}$ vector using an Invitrogen TOPO ${ }^{\mathrm{TM}} \mathrm{TA}$ Cloning ${ }^{\mathrm{TM}}$ Kit (Invitrogen, ThermoFisher Scientific, XX). The fragment was then digested from the TOPO vector, purified from agarose gel using the $\mathrm{Zymoclean}^{\mathrm{TM}}$ Gel DNA Recovery Kit (Zymo Research, Irvine, CA), and cloned into the Suc2 vector, pYST0 (Lee and Rose, 2012). Proper in-frame placement of the signal peptide sequence was verified through DNA sequencing (Eurofins, Louisville, KY). The resulting plasmid was subsequently named Suc2_MVLG_02245sp.

\begin{tabular}{|lr|}
\hline >MVLG_02245 Signal Peptide Protein Sequence \\
MTSQVRMQVESRAQRRAGAYASMRLLLALVFALCTLAHLPTTSAAPLASEQISSGLVFRQ \\
EPPRWLQFSRPHEKVSHQGKDHLDWKNTSPSPFTSSEPSRRVKRDEMWEQYIEGDEIDGE \\
KSEDVRAGDPDVAGDEVLTDTEIAGGADEAGEGSTGEKWWQARRRLRERRSATTRVVP
\end{tabular}

\section{Figure C3-1 The signal peptide region of MVLG_02245 predicted using SignalP4.1.}

The Suc2_MVLG_02245sp construct was transformed into the invertase-deficient (suc2-negative) Saccharomyces cerevisiae strain SEY 6210 (MATa leu2-3,112 ura3-52 his- $\Delta 200$ trp1- $\Delta 901$ lys2-801 suc2$\Delta 9$ GAL), using the Frozen-EZ yeast transformation II kit from Zymo Research, and plated onto both glucose and sucrose synthetic dropout media lacking Leucine (SDO Leu- $_{\text {- }}$. Colonies that grew on the Sucrose SDO plates were grown overnight in $3 \mathrm{~mL}$ of liquid Sucrose SDOLeu- media and plated onto both glucose and sucrose SDOLeu- assay plates in undiluted $\left(10^{7}\right.$ cells $\left./ \mathrm{mL}\right), 10 \mathrm{X}$ dilution $\left(10^{6}\right.$ cells $\left./ \mathrm{mL}\right)$, and $100 \mathrm{X}$ dilution $\left(10^{5}\right.$ cells $/ \mathrm{mL}$ ) concentrations. These assay plates were incubated at $30^{\circ} \mathrm{C}$ for 2 days before images were taken. To verify transformation, plasmids were re-extracted from the yeast colonies formed during the assay using a general yeast miniprep protocol (Protocols: Yeast Miniprep), transformed back into E. coli cells, and purified for repeat DNA sequencing to confirm the proper in-frame cloning of the construct (Eurofins, Louisville, KY). 


\subsection{Yeast Two-Hybrid (Y2H) assay of MVLG_02245 targets}

Using the NEBuilder HiFi DNA Assembly Master Mix kit, the fragment encoding the protein sequence for MVLG_02245 was cloned into the Y2H bait vector (pGBKT BD; Clontech, Mountain View, CA) without the signal peptide region (as determined by SignalP 4.1) and transformed into AH109 yeast cells using the Zymo Frozen EZ-Yeast Transformation II kit. The resulting transformants were mated against Y187 yeast cells containing the prey vectors bearing the cDNA library from S. latifolia infected with M. lychnidis-dioicae (Kuppireddy et al., 2017). The cDNA library in prey vector (pGAT7 AD, Clontech) was generated by CD Genomics (Shirley, NY, USA) as described previously (Kuppireddy et al., 2017). AH109 cells containing the MVLG_02245 bait vector were grown overnight in Trp- Single Dropout (SDO ${ }_{\text {Trp- }}$ ) liquid media. Cells were pelleted via centrifugation using a Labnet Hermle Z $233 \mathrm{M}-2$ centrifuge at 2,000 rpm and resuspended in $5 \mathrm{~mL}$ of $2 \mathrm{X}$ YPDA before being added to a $1 \mathrm{~L}$ flask along with $1 \mathrm{~mL}$ of Y187 cells containing the prey vectors and $45 \mathrm{~mL}$ of $2 X$ YPDA containing $50 \mu \mathrm{g} / \mathrm{mL}$ Kanamycin. Cells were then gently shaken at $45 \mathrm{rpm}$ on a platform shaker at $30{ }^{\circ} \mathrm{C}$ overnight to allow for mating. Successful mating was verified via microscopy (Figure C3-2) before cells were collected at 1,000 rpm, washed, and resuspended in $5 \mathrm{~mL}$ of sterile distilled water.

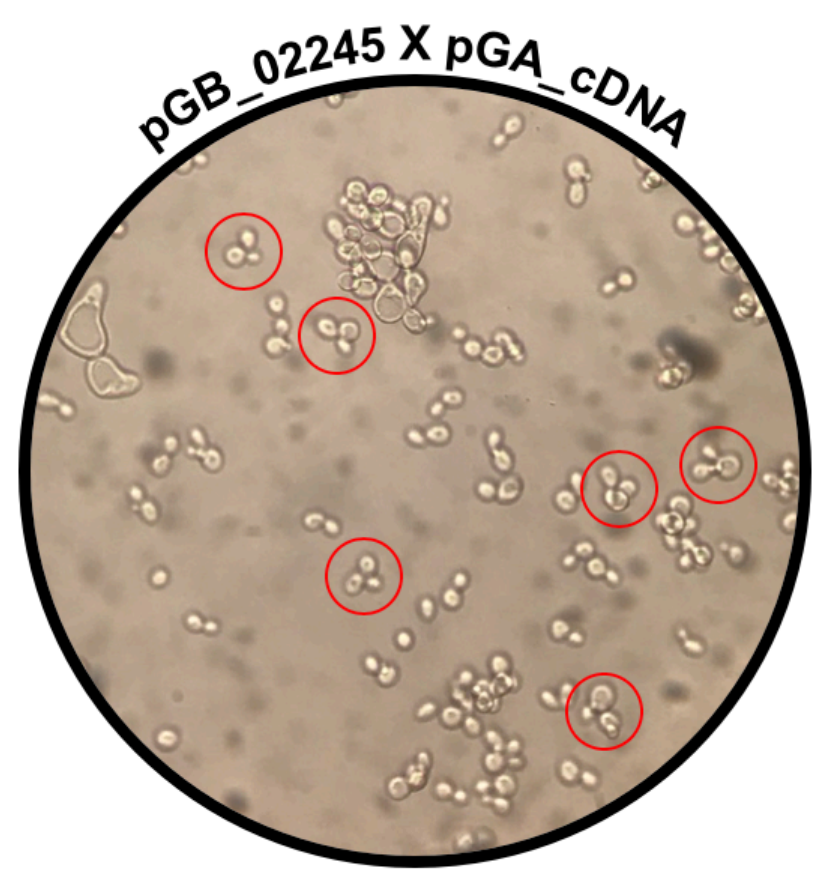

Figure C3-2 Mating verification between Saccharomyces cerevisiae cells. Expressing the pGB_02245 vector and cDNA library generated prey vectors. 
Mated cells were gently centrifuged with a Labnet Z233M-2 centrifuge at 1,000 rpm for $10 \mathrm{~min}$, and washed twice with diH2O before 1,000X and 10,000X dilutions were plated onto Trp- $\mathrm{Leu}^{-}$Double Dropout (DDOTrp- Leu-) plates and Trp- Leu` Ade- His` Quadruple Dropout (QDOTrp- Leu- Ade- His-) agar plates with $25 \mathrm{mM}$ 3AT (Sigma-Aldrich), x- $\alpha$-gal (Sigma-Aldrich), and $50 \mu \mathrm{g} / \mathrm{mL}$ Kanamycin. Both sets of plates were incubated at $30{ }^{\circ} \mathrm{C}$ for 3 days. Resulting blue colonies, indicating protein-protein interaction through the upregulation of galactase via localization of the DNA binding and activation domains, from the QDO $_{\text {Trp- }}$ Leu- Ade- His- plates were re-streaked onto QDOTrp- Leu- Ade- His- media containing $50 \mathrm{mM} 3 \mathrm{AT}$ to reduce growth due to leaky expression of the HIS3 gene that can confound screening for true interactions. Yeast minipreps to extract the bait and prey vectors from mated diploid cells were performed on colonies that grew on the 50 mM 3AT QDOTrp- Leu- Ade- His- plates. The plasmid mixtures were transformed via heat shock into competent DH5 $\alpha$ E. coli cells and plated onto LB agar containing $200 \mu \mathrm{g}$ ampicillin per $\mathrm{mL}$, to preferentially select for the prey vectors. These plates were incubated overnight at $37^{\circ} \mathrm{C}$ and plasmid DNA was extracted from resulting bacterial colonies. Such plasmids were sequenced (Eurofins) using primers upstream of the cDNA insertion. Sequencing results were then used in Blast searches for orthologs against the NCBI database to identify the host target. To verify true interactions, the bait and prey vectors for the MVLG_02245 coding region and each of its putative interaction partners were swapped using the NEBuilder HiFi DNA Assembly Master Mix, and mating was repeated and again confirmed via light microscopy (Figure C3-3) 

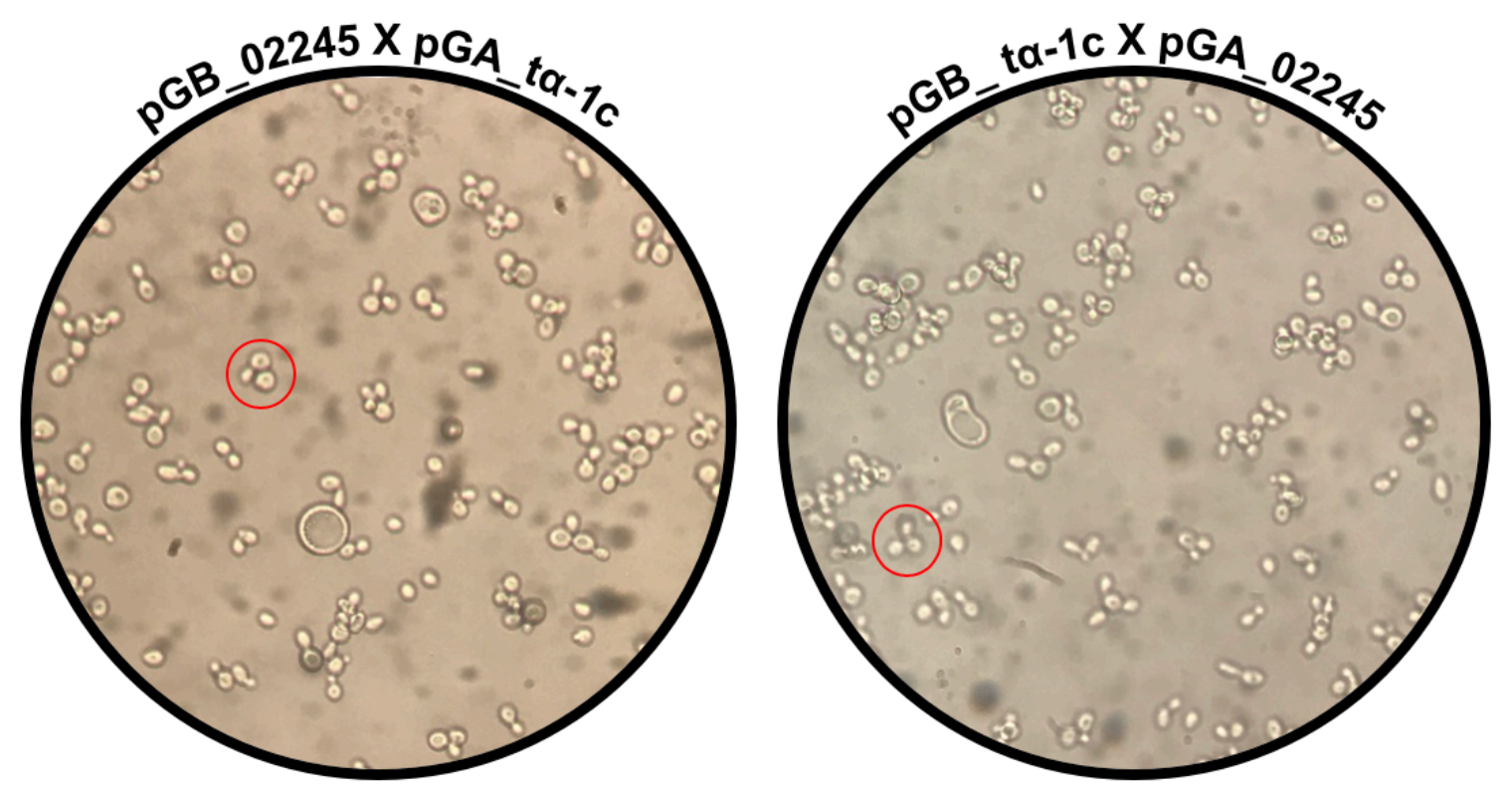

Figure C3-3 Repeat mating verification between Saccharomyces cerevisiae cells. Expressing the pGB_02245 vector and target match pGA_t $\alpha-1 \mathrm{c}$ from the cDNA prey library.

A spot assay for true interactors was prepared on both DDOTrp- Leu- and QDOTrp- Leu- Ade- His- media using undiluted (10E7 cells $/ \mathrm{mL}), 10 \mathrm{X}$ dilution $(10 \mathrm{E} 6 \mathrm{cells} / \mathrm{mL})$, and $100 \mathrm{X}$ dilution $(10 \mathrm{E} 5 \mathrm{cells} / \mathrm{mL})$ concentrations of cell suspension. As a control, single vector colonies and mated controls were similarly prepared and spotted DDOTrp-Leu- assay plates were incubated at $30{ }^{\circ} \mathrm{C}$ for 2 days, while the QDOTrp-Leu-AdeHis- assays were incubated at $30^{\circ} \mathrm{C}$ for 4 days before images were taken.

\section{Results}

\subsection{Local BLAST results for predicted MVLG_02245 coding sequence}

Local BLAST alignment of the DNA and protein sequence for MVLG_02245 demonstrated that the coding sequence for MVLG_02245 is conserved across multiple species of Microbotryum (Figure 3). While amino acid sequence was conserved between the sister species M. lychnidis-dioicae (MVLG/MvSl) and M. silenesdioicae (MvSd), with only 4 amino-acid substitutions resulting from 6 nucleotide substitutions, the protein sequence was more different in the distantly related M. violaceum var. paradoxa (MvSp), with 29 amino acid substitutions resulting from 51 nucleotide substitutions (Figure C3-4, Supplemental Table C3-1), when 
compared to M. lychnidis-dioicae. No insertions or deletions, and subsequently no frame shifts, were observed in the MVLG_02245 gene in any of the three species.

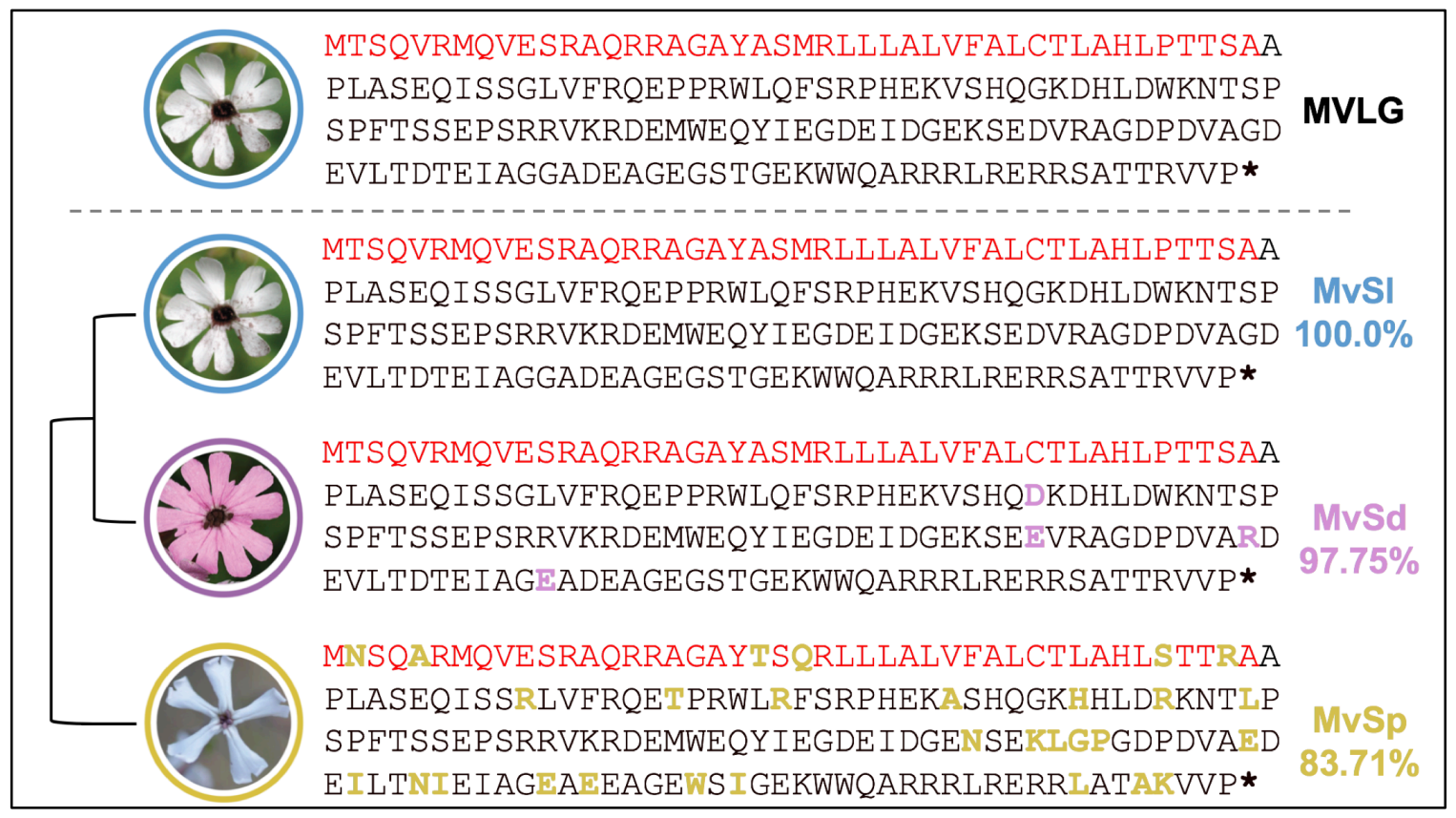

Figure C3-4 BlastP comparison between three species of Microbotryum for MVLG_02245. The

sequence for MVLG_02245 retrieved from JGI was compared to that from the PacBio assembly for the same strain (MvSl), as well as the PacBio assemblies for its sister species (MvSd) and a more distantly related species of Microbotryum (MvSp). Amino acid substitutions, with reference to the MvSl strain, are shown in bolded letters color coded by their species (pink for MvSd, gold for MvSp).

When compared to the amino acid sequence for MVLG_02245 in M. lychnidis-dioicae, the M. silenesdioicae and $M$. violaceum var. paradoxa orthologues only shared one amino acid substitution, $\mathrm{G}_{146}->\mathrm{E}_{146}$. Interestingly, while there were no differences in the signal peptide region for MVLG_02245 found in M. lychnidis-dioicae relative to M. silenes-dioicae, the corresponding region of M. violaceum var. paradoxa had 6 substitutions out of 44 amino acids for this region (Figure C3-4). 
3.2 Predicted secretion, function, and Expression of the MVLG_02245 effector

Screening of MVLG_02245 against SignalP 4.1 indicated that MVLG_02245 is predicted to be secreted (Supplemental Table C3-2), which is in accord with previous findings by Perlin et al., (2015); Toh et al., (2018). RNASeq further demonstrated that mRNA is present for MVLG_02245 and upregulated during infections in the floral and floral stem tissues of the host (Table 1; Perlin et al., 2015; Toh et al., 2018).

Table C3-1: Expression of MVLG_02245 on various media and in planta, presented as TPM ${ }^{\mathrm{a}}$

\begin{tabular}{|ccc|cccc|c|}
\hline Water & $\begin{array}{c}\text { Rich } \\
\text { Media }\end{array}$ & $\begin{array}{c}\text { Mating } \\
\text { on } \\
\text { Nutrient- } \\
\text { limited } \\
\text { Medium }\end{array}$ & $\begin{array}{c}\text { Male } \\
\text { Infected } \\
\text { Tissue: } \\
\text { Stage 8 }\end{array}$ & $\begin{array}{c}\text { Male } \\
\text { Infected } \\
\text { Tissue: } \\
\text { Stage 9 }\end{array}$ & $\begin{array}{c}\text { Male } \\
\text { Infected } \\
\text { Tissue: } \\
\text { Stage 10 }\end{array}$ & $\begin{array}{c}\text { Male } \\
\text { Infected } \\
\text { Tissue: } \\
\text { Late }\end{array}$ & $\begin{array}{c}\text { Male } \\
\text { Infected } \\
\text { Stem } \\
\text { Tissue }\end{array}$ \\
\hline 1 & 5 & 1.13 & $\mathbf{7 3 . 2 3}$ & $\mathbf{5 3 . 6 5}$ & $\mathbf{4 1 . 8 8}$ & 4.98 & $\mathbf{3 0 . 0 6}$ \\
\hline$\square$ & $\square$ & $\square$ & 仓 & 仓 & ن & $\square$ & U \\
\hline
\end{tabular}

a - transcripts per million; Male-infected Stem tissue, male-infected floral stem tissue. 2-3 independent determinations via RSEM.

However, while the MVLG_02245 gene in M. lychnidis-dioicae and M. silenes-dioicae is predicted to be secreted, the amino acid substitutions observed in the signal peptide region of MVLG_02245 for $M$. violaceum var. paradoxa are not predicted to abrogate secretion of the protein (Supplemental Table C3-1). To predict whether the MVLG_02245 protein may play a role in manipulating the host, the amino acid sequences for all three species of Microbotryum were run against the online effector prediction software, EffectorP 1.0 (Sperschneider et al., 2015). The MVLG_02245 protein was predicted to be an effector in all three species, with a probability score of $0.865,0.686,0.645$ for M. lychnidis-dioicae, M. silenes-dioicae, and M. violaceum var. paradoxa, respectively (Supplemental Table C3-2). To screen for any shared sequence with known effectors, we used the Pfam 32 and HMMER 3.1b1 tools to screen for protein families; however, neither of the programs yielded any significant results (Supplemental Table C3-2).

The predicted effector function in all three species, the combination of predicted secretion in the sister species pair, and the lack of a Pfam domain indicates that MVLG_02245 is likely an effector with a unique function for the infection of Silene hosts, specifically that of the sister species given its lack of 
mobilization in $M$. violaceum var. paradoxa. Evolutionarily speaking, this could either indicate genetic drift in the gene due to a lack of use in the $S$. paradoxa host, or represent positive selection of the effector in different evolutionary trajectories since divergence of $M$. violaceum var. paradoxa from the sister-species progenitor. Prediction that MVLG_02245 is a genus-specific effector for Microbotyrum is further supported by a lack of blastn and blastp hits to sequence outside the Microbotryum genus when screened against the general NCBI genome database. Furthermore, MVLG_02245 is predicted to be a disordered protein (Figure C3-5). Intrinsically disordered proteins allow for flexibility and have been described as another hallmark for small-secreted effectors for various pathogens (Marín et al., 2013), including in Microbotryum as outlined by Kuppireddy et al., (2015).

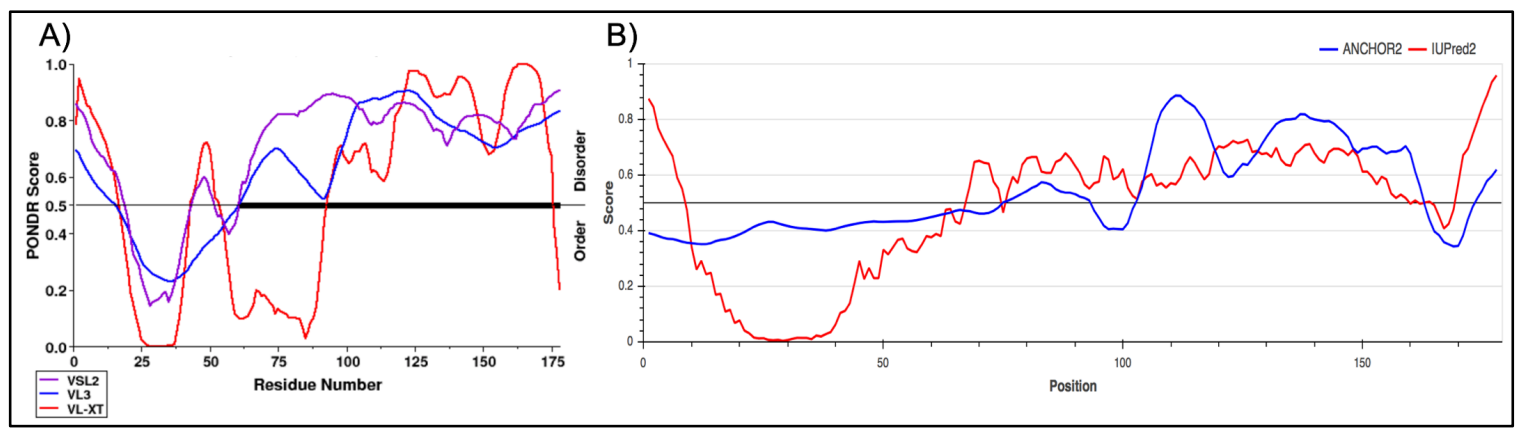

Figure C3-5 Prediction of disorder in the protein sequence for MVLG_02245. using A) PONDR and B) IUPred2A.

\subsection{Yeast Secretion Trap results}

The capacity for the M. lychnidis-dioicae and M. silenes-dioicae MVLG_02245 signal peptide to signal for protein secretion was confirmed through YST testing of the signal peptide region, predicted by SignalP 4.1 (Figure 5). SEY cells transformed with the Suc2_MVLG_02245sp vector were able to secrete the invertase enzyme to breakdown sucrose and survive on sucrose as a sole carbon source (Figure C3-6). 


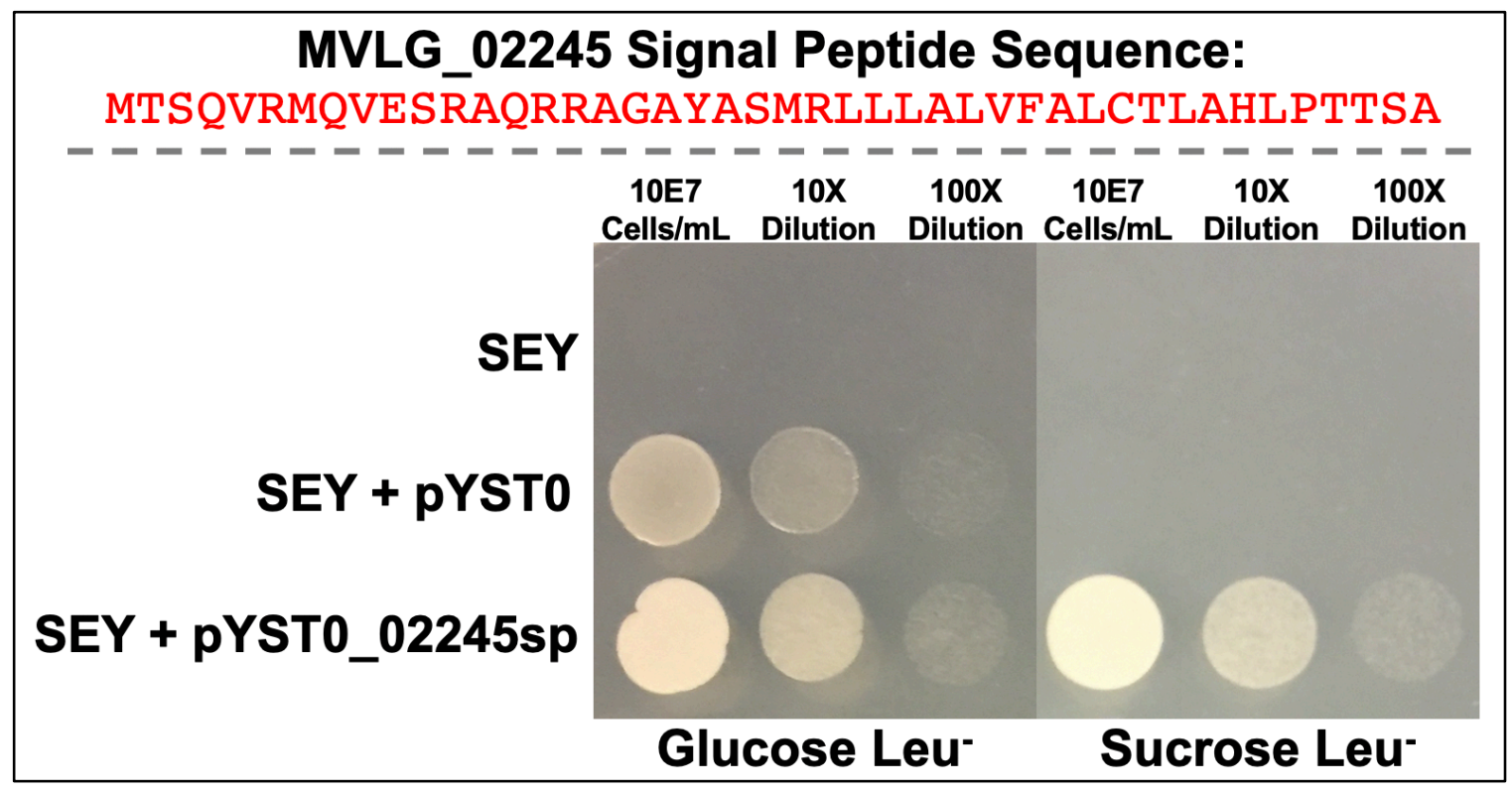

Figure C3-6 Yeast Secretion Trap results for the signal peptide region of MVLG_02245. The figure shows yeast colonies after 2 days of growth on Glucose Leucine Dropout Media, left, and Sucrose Leucine Dropout Media, right. In the top row are untransformed SEY strain cells of Saccharomyces cerevisiae. The second row contains SEY cells transformed with just the pYST0 vector. The third row contains SEY cells transformed with the pYST0 vector containing the signal peptide from MVLG_02245, as predicted by SignalP 4.0, cloned upstream and in-frame of the invertase enzyme. The signal peptide sequence is shown in red above the spotting assay.

\subsection{Host targets of the MVLG_02245 effector in Silene latifolia}

With an abundance of bioinformatic evidence suggesting that MVLG_02245 is a secreted effector in the Microbotryum genus, we tested the M. lychnidis-diociae variant for a host target using a Y2H approach. Y2H mating assays between AH109 cells containing the MVLG_02245 bait vector with Y187 cells containing the infected plant tissue cDNA prey vector library yielded blue colonies on $50 \mathrm{mM}$ 3AT QDOTrp- Leu- Ade- Hismedia. Plasmid extraction followed by sequencing for 50 of these diploid colonies demonstrated that four particular proteins were found more predominantly than others. The sequences for these identified plant proteins were compared against the NCBI database and yielded plant orthologs for a ferredoxin-thioredoxin reductase catalytic chain protein, a Photosystem II protein, a xyloglucan endotransglucosylase/hydrolase 4 protein, and a Tubulin $\alpha-1$ chain protein (t $\alpha-1 \mathrm{c})$. Only the fourth interaction, MVLG_02245 X t $\alpha-1 \mathrm{c}$, yielded 
blue colonies when the coding regions were swapped between the bait and prey vectors and mating was repeated (Figure 6). While the interaction between MVLG_02245 and t $\alpha-1 \mathrm{c}$ was confirmed in the vector swap, the resulting diploids grew slower in both the DDO and QDO media (Figure C3-7).

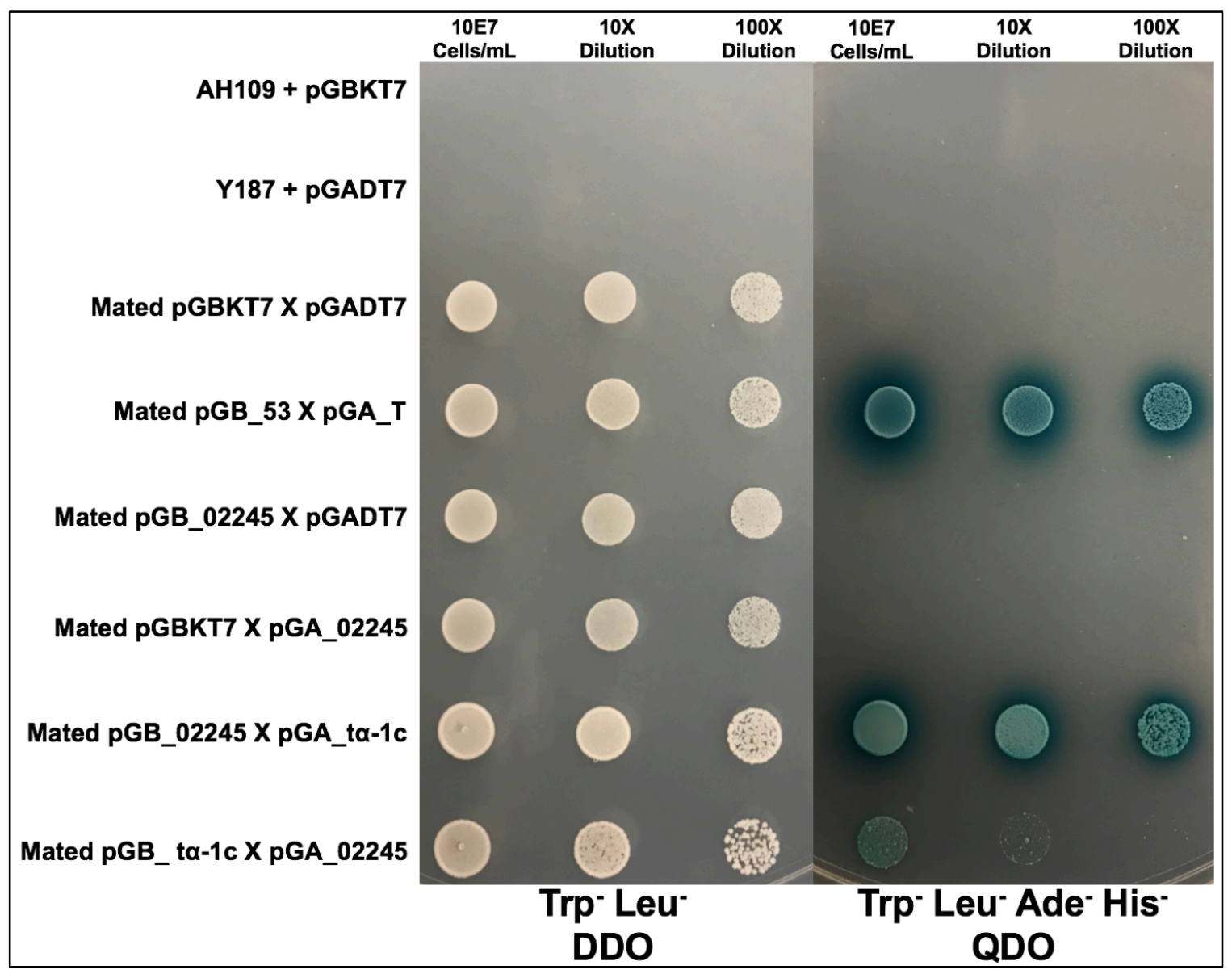

Figure C3-7 Yeast two-hybrid mating results between MVLG_02245 and ta-1c. Colonies are shown after two days of growth on DDO, left, and 4 days of growth on QDO, right. A series of negative controls were used including the AH109 yeast strain transformed with an empty bait vector (pGBKT7), top row, the Y187 strain transformed with an empty prey vector (pGADT7), second row, Diploid offspring of mated strains containing both the empty bait and empty prey vectors, third row, Diploid cells containing the MVLG_02245 bait vector and the empty prey vector, fifth row, and Diploid cells containing the empty bait vector with the t $\alpha-1 \mathrm{c}$ prey vector, sixth row. Diploid cells containing the bait and prey vectors for known strong interactors p53 and T-antigen were used as a positive control in the fourth row. Diploid cells containing 
the MVLG_02245 bait vector and ta-1c prey vector are spotted in the seventh row, and diploid cells containing the swapped t $\alpha-1 \mathrm{c}$ bait vector and MVLG_02245 prey vector are spotted in the seventh row.

\section{Discussion}

Our findings demonstrate that, despite a difference in annotation, MVLG_02245 is indeed a secreted protein which likely acts as an effector utilized by M. lychnidis-dioicae during infection of its host, Silene latifolia. Interestingly however, the limited number of differences observed in the predicted secretion signal peptide sequences between the MVLG_02245 gene of the two sister species, M. lychnidis-dioicae and M. silenesdioicae, compared with the more extensive changes for the corresponding region found in the more distantly related M. violaceum var. paradoxa, mirrors the host specificity that is observed between the three groups. While M. lychnidis-dioicae and M. silenes-dioicae perform much better at infecting their own hosts, $S$. latifolia and S. dioica, respectively, they are able to infect each other's hosts, albeit to a reduced degree compared to their natural hosts (de Vienne et al., 2009; Putten et al., 2003). This is in contrast to M. violaceum var. paradoxa, which is unable to infect either of the others' hosts, and vice versa (de Vienne et al., 2009). These observations are consistent with findings from Beckerson et al. (2019), where "SP-Mixed" groups were identified in which all three species contained orthologs for certain effectors, but only one or two species in the 3-way comparison were predicted to secrete the protein (Beckerson et al., 2019). These SP-Mixed groups are suspected to play a role in host-specificity through "mobilization" or loss of secretion in proteins (Beckerson et al., 2019).

While our Y2H results have identified an interaction partner for the MVLG_02245, a tubulin $\alpha-1$ chain protein ortholog, its exact purpose for binding to the protein in planta still remains unclear. These tubulin proteins act as a linker in microtubule production in the host, and therefore MVLG_02245 may act to prevent tubulin $\alpha-1$ chain proteins from linking with their $\beta$ chain counterparts (Hashimoto, 2015). Doing so can disrupt structural arrangement of the plant cell cytoskeleton, mitosis, and other microtubulebased processes, which can have a wide variety of effects in the plant ranging from structural instability to disruption of microtubules that traffic vesicles vital for the plant immune response (Büttner, 2016). Experiments performed on effectors targeting tubulin chains by Lee et al., secreted by the bacterial phytopathogen Pseudomonas syringae $p v$. tomato, demonstrate an ability for these proteins to destabilize 
microtubules and interfere with the host's secretion of reporter proteins to the apoplast (Lee et al., 2012). These microtubules also play a role in cell division, and their disruption may play a role in regulating growth of the host plant. Thus, it is conceivable that MVLG_02245 may ultimately weaken both the plant cell and the host immune response, making the host more susceptible to penetration by the fungi. The upregulation of MVLG_02245 in the floral stem tissue and its downregulation in late-stage infection of floral tissue of the host lends even more credence to the effector's use during primary infiltration and establishment in the plant.

To verify and further expand on these predictions, future studies will utilize GFP tagged MVLG_02245 for localization studies of the secreted protein within the plant host. To prepare for this work, the coding sequence for MVLG_02245 was cloned into the Agrobacterium-mediated transformation (ATMT) vector for M. lychnidis-dioicae, pMvHyg (Toh et al., 2017), using the NEBuilder HiFi DNA Assembly Master Mix kit, along with both the constitutively expressed promoter MVLG_05589-P and the coding sequence for GFP, fused in-frame to the MVLG_2245 coding region along with an intervening $2 \mathrm{X}$ Gly-Gly-Ser residue linker. The resulting construct, pMvHyg_MVLG_02245-GFP (Figure C3-8), was transformed into EHA105 Agrobacterium cells using electroporation $(2.5 \mathrm{kV}, 400$ ohms and $25 \mu \mathrm{F})$ and transformants were selected on LB agar-containing $50 \mu \mathrm{g}$ kanamycin/mL. Successful transformants were restreaked onto LB containing $50 \mu \mathrm{g}$ kanamycin and $100 \mu \mathrm{g}$ spectinomycin per $\mathrm{mL}$ agar to ensure that both the pMvHyg and Helper plasmids were in the cells. Transformed EHA105 cells were further confirmed through colony PCR, before being used to transform M. lychnidis-dioicae using the ATMT protocol developed by Toh et al., (2016). 


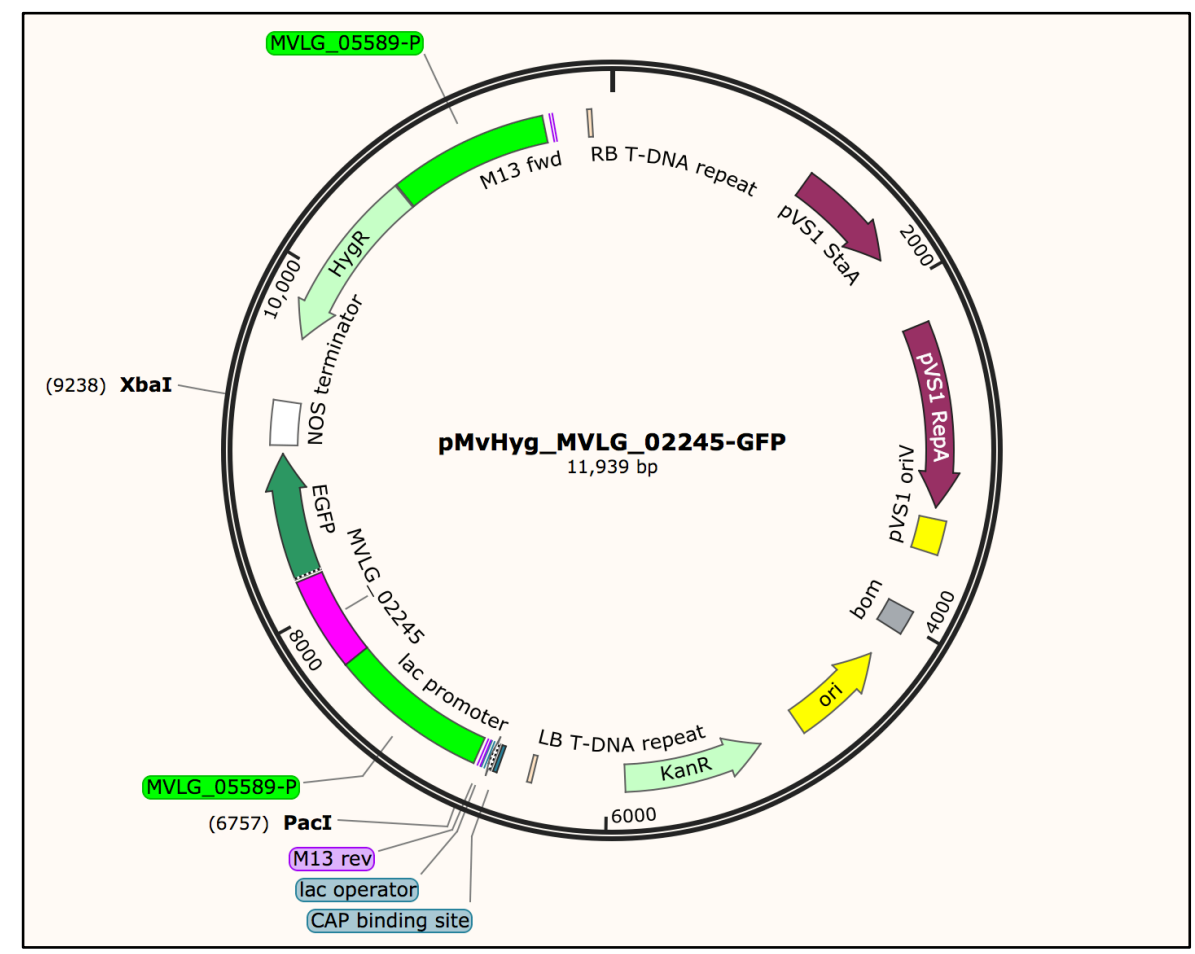

Figure C3-8 SnapGene image of pMvHyg_MVLG_02245-GFP.pMvHyg_MVLG_02245-GFP contains the Microbotryum lychnidis-dioicae gene MVLG_02245 fused in-frame at the 3' end with the GFP coding region. Expression of the MVLG_02245-GFP protein is driven by the constitutively expressed promoter. All of these components are cloned in-between the left and right border of the Agrobacterium-mediated transformation vector, pMvHyg. pMvHyg (Toh et al., 2017) also contains a hygromycin resistance gene included within the transfer region, also driven by the MVLG_05589-P promoter, to select for transformants after integration into the M. lychnidis-dioicae genome. (https://www.snapgene.com/snapgene-viewer/).

Prior to ATMT, the M. lychnidis-dioicae p1A1 strain was grown on yeast peptone dextrose agar with $10 \%$ dextrose (YPD- $10 \%$ ) for 2 days at $24^{\circ} \mathrm{C}$. Cells were resuspended in $1 \mathrm{~mL}$ of induction medium broth and diluted to an $\mathrm{OD}_{600}$ of approximately $10 \mathrm{E} 7 \mathrm{cells} / \mathrm{mL}$. The same was done for the transformed EHA105 cells, approximately 10E8 cells/mL. A 1:1 ratio Microbotryum to Agrobacterium cells was mixed together and spotted onto IM plates using $100 \mu \mathrm{L}$ of each cell suspension before being incubated at room temperature for 3 days, after which the resulting mass of cells were retrieved from the plates using sterile plastic loops and resuspended in $600 \mu \mathrm{L}$ of YPD-10\% liquid media. $200 \mu \mathrm{L}$ of each suspension were then 
spread onto YPD-10\% containing $150 \mu \mathrm{g} / \mathrm{mL}$ Hygromycin B containing $100 \mu \mathrm{g} / \mathrm{mL}$ Cefoxitin plates. These plates were incubated at $24^{\circ} \mathrm{C}$ for 14 days. Resulting transformants were re-streaked onto YPD- $10 \%$ containing $150 \mu \mathrm{g} / \mathrm{mL}$ Hygromycin B and used for genomic extractions and PCR screening for successful integration of the transfer plasmid. Verified transformants were used for confocal imaging of GPF and infection of S. latifolia to observe cellular localization of the MVLG_02245 in the host.

The findings in this research not only provide a plausible characterization for one of the many conserved core secreted proteins in the Microbotryum genus, but also provide an example of the necessity for molecular genetic studies to corroborate bioinformatic predictions. Bioinformatic predictions and functional analyses are therefore synergistic and intertwined, neither of which can be as productive without the other. Furthermore, because there are many atypical mechanisms at work in various species, especially those not as well defined as model organisms, caution should be taken when eliminating candidate genes from annotations. While predictive models are essential for initial work involving genome characterization, these models should be just that, a starting point for an eventual complete and more inclusive analysis. 


\section{CHAPTER 4}

\section{ASSESSING THE ROLE OF SPECIES-SPECIFIC EFFECTORS IN HOST- SPECIALIZATION}

\section{Introduction}

While gradual stepwise changes to the amino acid sequence of core secreted proteins (SPs) can lead to coevolution between a pathogen and its hosts, the emergence of novel SPs could lead to rapid evolutionary changes. The acquisition of novel secreted proteins, in this sense defined by an effector for which the host has no defensive response, can arise from a variety of biological processes including horizontal gene transfer (Casa-Esperón, 2012), sexual recombination (Grigg \& Suzuki, 2003), mobilization of a previously nonsecreted protein (Beckerson et al., 2019), and gene duplication (Andersson, Jerlström-Hultqvist, \& Näsvall, 2015). While the emergence of a new effector can give the pathogen an immediate edge in the evolutionary tug-of-war with its host, we would expect the hosts to adapt over time to new effectors in a coevolution model. Therefore, unique SPs may act as evolutionary milestones and provide some insight into the divergence of species over time. Furthermore, these novel proteins may play a pivotal role in the pathogenicity of different species, contributing immensely to host specificity among pathogens separated by post-zygotic barrier, such as is seen in the Microbotryum genus. In this investigation, we identify secreted proteins unique to the two sister taxa of Microbotryum, M. lychnidis-dioicae and M. silenes-dioicae, and initiate experiments to evaluate their roles in pathogenicity on each host plant. To do so, we utilized a bioinformatic approach to compare eight annotated Microbotryum genomes for unique proteins with hallmarks of secretion, verified by parameters set in Beckerson et al., 2019. Finally, to test the role of speciesspecific effector in pathogenicity, a subset of these identified species-specific effector candidates were heterologous expressed in each sister species and infection assays were performed. 


\section{Materials and Methods}

2.1 Identifying species-specific small secreted proteins

To identify small secreted proteins specific to individual species from the Microbotryum genus, a comparative secretomics analysis was performed on 8 species for which genomic annotation was available, provided by Dr. Ricardo de la Vega and Dr. Tatiana Giraud. To identify small secreted proteins in each of the 8 species, a pipeline following criteria from Beckerson et al., 2019 (see Chapter 2) was used to identify proteins with hallmarks of secretion as evidenced by in their protein leader sequence and to rule out potential cellular localization of proteins in organelles or the cellular membrane (Beckerson et al., 2019). Proteins that met these criteria were further screened against using 4 more secretomics tools to corroborate predicted secretion (Beckerson et al., 2019). To be considered for this study, proteins with predicted secretion had to have passed the initial selection with SignalP4.0, passed all criteria for non-cellular localization (TMHMM, Phobius, Prosite, PredGPI, NucPred), and pass $3 / 4$ of the final 4 cutoff values for predicted secretion (Phobius, TargetP, SingalP3.0) (Figure C4-1). The resulting lists for each species were then blasted against the proteomes of the other 7 species to rule out any orthologous genes and identify species-specific genes. As demonstrated by Beckerson et al., 2019, some secreted proteins in one species may have non-secreted orthologs in another. Therefore, to identify truly unique species-specific secreted proteins, and to rule out those mobilized by selective pressures, only proteins with no orthologous match were used in this study. Furthermore, only amino acid sequences beginning with methionine were considered true candidate proteins for this study. For the sister species under analysis, M. lychnidis-dioica (MvSl) and M. silenes-dioicae (MvSd), the resulting list of unique secreted proteins was further screened for proteins smaller than 250 amino acids, resulting in a workable list of 3 unique proteins for MvSl and 5 for MvSd (Figure C4-1). 


\section{Computational Framework for Determining Species-Specific Small Secreted Proteins}

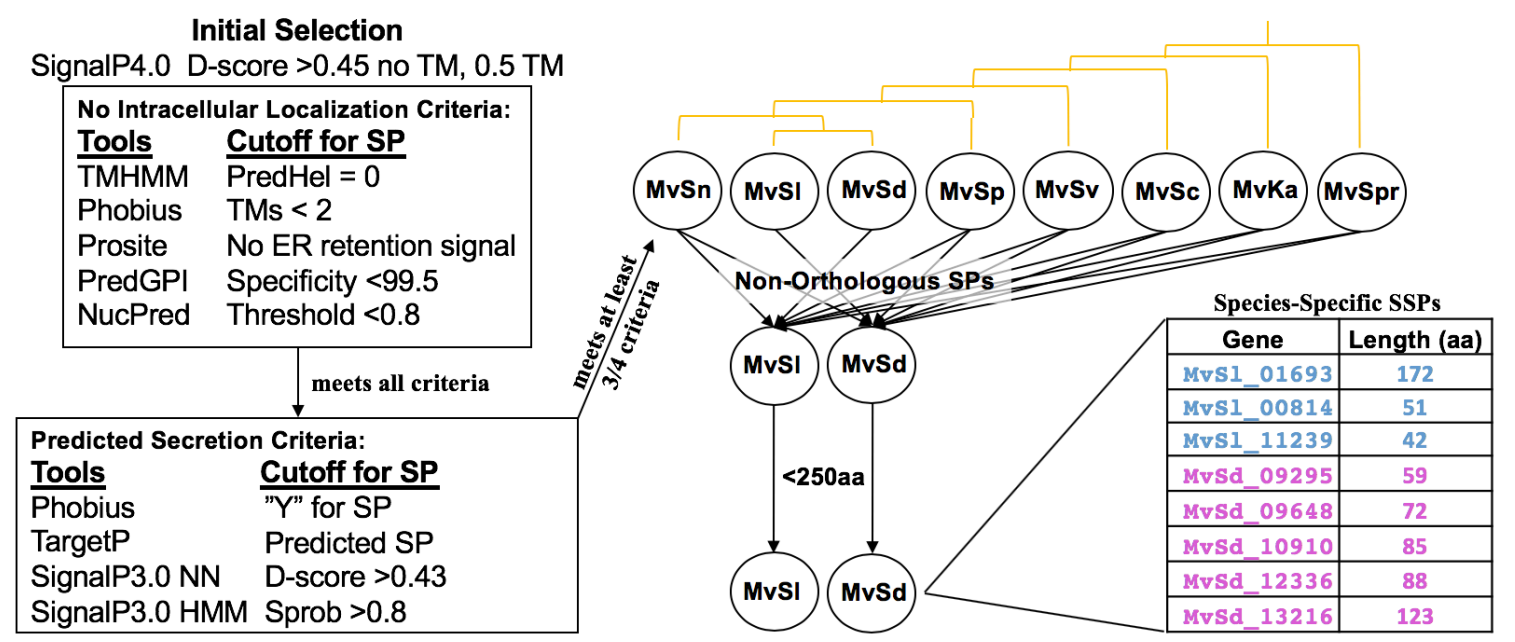

Figure C4-1 Computational framework for identification of species-specific SSPs. The secretomes for M. lychnidis-dioicae (MvSl) and M. silenes-dioicae (MvSd) were determined in Beckerson et al., 2019 (see Chapter 2) using the 10 tools and cutoff values listed on the left of the diagram. SignalP4.0 was used to identify a list of secreted proteins (SPs) from the entire genome. These putative SPs were then analyzed for intercellular localization using TMHMM, Phobius, Prosite, PredGPI, and NucPred. To increase certainty that putative SPs are indeed secreted, the remaining list was run through Phobius TargetP1.0, and SignalP3.0, and must have passed at least 3 of the 4 cutoff values to be considered a candidate for this study. The resulting SPs for each sister species were then compared against each other, along with 6 additional distantly related species of Microbotryum (M. violaceum sensu stricto on Silene nutans [MvSn], M. sensu lato on S. paradoxa [MvSp], M. lagarehemi on S. vulgaris [MvSv], M. violaceum sensu lato on S. carolineana [MvSc], M. lscabiosae on S. vulgaris [MvKa], M. intermedium on S. pratensis [MvSpr]), to identify SPs without orthologs in other species, i.e., species-specific SPs. Any SPs with more than 250 amino acids were then removed to generate the final list of species-specific small secreted proteins.

\subsection{EffectorP and gene expression}

The list of species-specific small secreted proteins, 3 for M. lychnidis-dioicae and 5 for M. silenes-dioicae, were further analyzed using the EffectorP 1.0 program. EffectorP is a machine learning tool used to predict whether a secreted protein is an effector based on a pool of data for known plant effectors. The tool looks for patterns found among the sample set and compares these to the submitted amino acid sequence 
(Sperschneider et al., 2015). The program then outputs a predictive number between 0 and 1 to represent a percent certainty that the secreted protein is an effector, as predicted by the program. A value over $50 \%$ is considered likely an effector. From the results for all 8 amino acid sequences tested, two candidates were selected, one from each species, with the highest likelihood of acting as an effector based on predictive measures (Table C4-1).

Table C4-1. EffectorP 1.0 Results for Species-Specific SPs

\begin{tabular}{|c|c|l|}
\hline Protein Sequence & $\begin{array}{c}\text { EffectorP 1.0 } \\
\text { Likelihood }\end{array}$ & \multicolumn{1}{|c|}{ Amino Acid Sequence } \\
\hline MvS1_01693 & Effector $(0.585)$ & MQRAICHLPSPPHWVSAPRHRNTTGDHVTQSSHRSGELLQNNTDRYFF \\
& & GCFDSRNVWIATRGVLCNALGCDTIDDADYTTLKRLLFCQKGITLKAK \\
& & LSDQEGAGRTIKIFTGMGLEMISSGRGRMQKHGTRMESVERKRVVLEE \\
& & GMIHRSFINSTKLQKRWRDREGQRHTLLEKRRNASKVEQEASAAERYP \\
& & KRVMYGHFGAPEDEEKKKQGTVGGAIANGNANAD \\
\hline MvSd_09295 & Effector (0.935) & MVCATWFVFVSGRFTLTLVKKKCCGPPGRLWWMSQHTVVSAAPACG \\
& & VHVPIRSHVQALW \\
\hline
\end{tabular}

From the initial screening, the M. lychnidis-dioicae gene MvSl_01693 with an effector likelihood of 0.585 was selected for heterologous expression in M. silenes-dioicae, and the M. silenes-dioicae gene MvSd_09295 with an effector likelihood of 0.935 was selected for heterologous expression in M. lychnidis-dioicae. While expression data is not available for the expression of M. silenes-dioicae genes, MvS1_01693 was aligned with its counterpart in the expression data available for M. lychnidis-dioicae at the BROAD institute (Perlin et al., 2015; Toh et al., 2017) to check for further evidence that the selected secreted protein is upregulated during infection and therefore a likely effector (Table C4-2). 
Table C4-2: Expression of MvSl_01693 on various media and in planta, presented as TPM ${ }^{\mathrm{a}}$

\begin{tabular}{|ccc|cccc|c|}
\hline Water & $\begin{array}{c}\text { Rich } \\
\text { Media }\end{array}$ & $\begin{array}{c}\text { Mating } \\
\text { on } \\
\text { Nutrient- } \\
\text { limited } \\
\text { Medium }\end{array}$ & $\begin{array}{c}\text { Male } \\
\text { Infected } \\
\text { Tissue: } \\
\text { Stage 8 }\end{array}$ & $\begin{array}{c}\text { Male } \\
\text { Infected } \\
\text { Tissue: } \\
\text { Stage 9 }\end{array}$ & $\begin{array}{c}\text { Male } \\
\text { Infected } \\
\text { Tissue: } \\
\text { Stage 10 }\end{array}$ & $\begin{array}{c}\text { Male } \\
\text { Infected } \\
\text { Tissue: } \\
\text { Late }\end{array}$ & $\begin{array}{c}\text { Male } \\
\text { Infected } \\
\text { Stem } \\
\text { Tissue }\end{array}$ \\
\hline 0.04 & 0 & 0.02 & 0 & 7.5 & 0 & 489.16 & 397.24 \\
\hline$\square$ & $\square$ & $\square$ & $\square$ & $\square$ & $\square$ & ن & U \\
\hline
\end{tabular}

a - transcripts per million; Male-infected Stem tissue, male-infected floral stem tissue. 2-3 independent determinations via RSEM.

Despite its marginal qualification in the predictive effector program EffectorP 1.0, significant upregulation in late infection in both floral and floral stem tissue along with no expression in rich media, water agar, or during mating, indicate that the MvS1_01693 secreted protein may play a role in infecting its Silene latifolia host, making it an ideal candidate for expression in M. silenes-dioicae.

\subsection{Agrobacterium-mediated transformation of MvSd_09295 and MvSl_01693}

The two genes encoding the species-specific small secreted proteins, MvSl_01693 and MvSd_09295, were selected for heterologous expression in the sister species. To express each gene in the other species, the reliable Agrobacterium-mediated transformation protocol by Toh et al., 2016 was used. Because there is no expression data available for $M$. silenes-dioicae during any stage of its lifecycle, and because the sister species are so closely related, the endogenous promoters for each gene, which we defined as the $1 \mathrm{~kb}$ sequence preceding the methionine codon, and the transcriptional termination sequence, which we defined as the $1 \mathrm{~kb}$ sequence following the stop codon, were used to drive heterologous expression of the species-specific genes in effort to preserve proper time-specific transcription and to rule out any phenotypic differences that may be attributed to over expression of the gene if a constitutive promoter was used instead. Thus, the coding region along with $1 \mathrm{~kb}$ upstream and downstream elements were amplified from host genomic DNA and cloned into the pMvHyg (Toh et al., 2016) vector using the NEBuilder ${ }^{\circledR}$ \#E2621 HiFi DNA Assembly Master Mix Kit for transformation into Agrobacterium (Figure C4-2). 


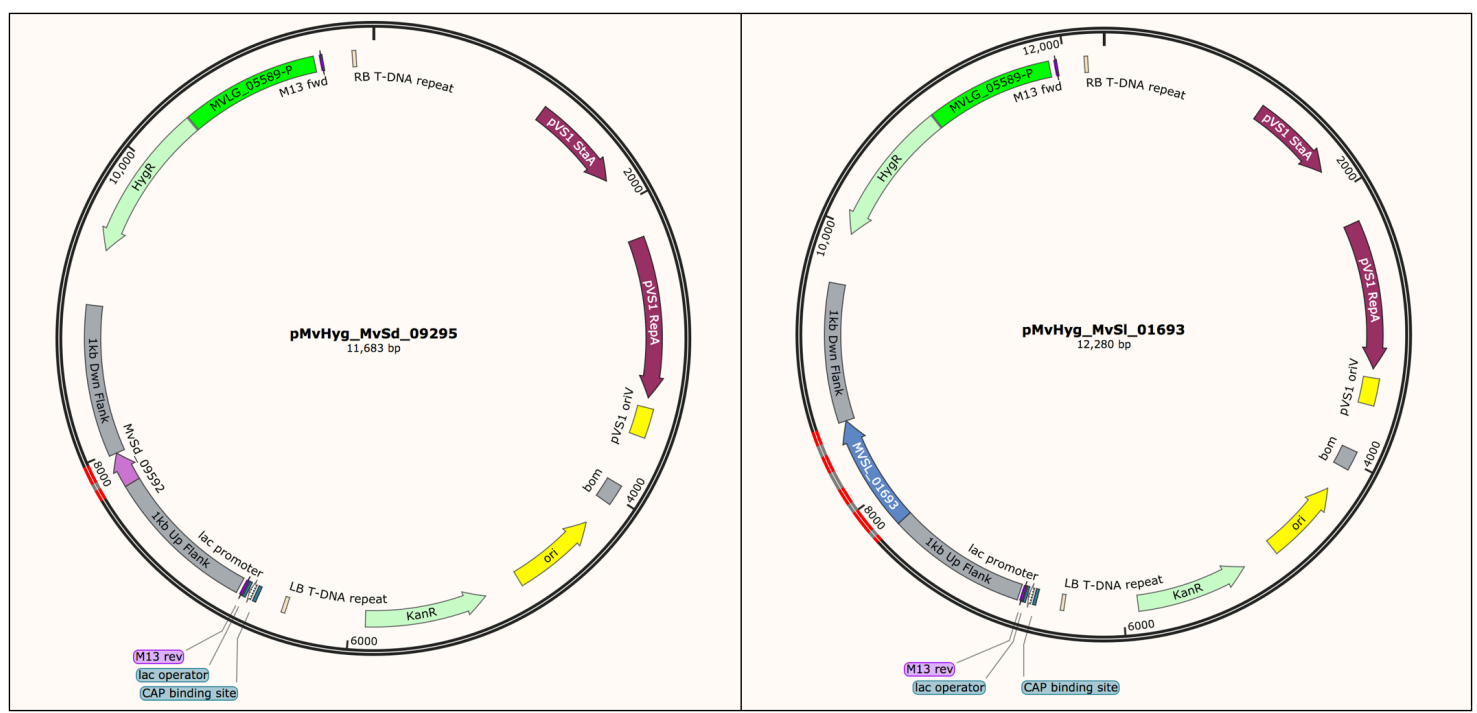

Figure C4-2 Snap gene image of the vectors for heterologous expression of species-specific genes.

pMvHyg_MvSd_09295, left, was generated via cloning of the MvSd_09295 gene into pMvHyg from Toh et al., 2016 for expression in MvSl. Likewise, pMvHyg_MvS1_01693, right, was generated via cloning of the MvSl_01693 gene into pMvHyg for expression in MvSd.

Each respective plasmid was transformed into EHA105 Agrobacterium cells using electroporation (2.5 kV, $400 \mathrm{ohms}$ and $25 \mu \mathrm{F}$ ) following the protocol outlined in Toh et al., (2016). Putative transformants were selected on LB agar-containing $50 \mu \mathrm{g}$ kanamycin/mL and then re-streaked onto LB agar containing 50 $\mu \mathrm{g}$ kanamycin/ and $100 \mu \mathrm{g}$ spectinomycin per $\mathrm{mL}$ agar to ensure that both the pMvHyg containing the respective species-specific gene and the helper plasmids were in the cells. Surviving EHA105 cells were further confirmed through colony PCR, before being used to transform the M. lychnidis-dioicae cells with pMvHyg_MvSd_09295 and the M. silenes-dioicae cells with pMvHyg_MvS1_01693. To transform their respective Microbotryum species, 1E7 cells/mL of each mating type for both Microbotryum species and each transformed Agrobacterium strain were measured using spectrophotometry (Microbotryum: $\mathrm{OD}_{600}$ 1 = 3.4E7 cells/mL; Agrobacterium: $\mathrm{OD}_{600} 1=8 \mathrm{E} 8$ cells $/ \mathrm{mL}$ ) and mixed in equal volumes. $200 \mu \mathrm{L}$ of each suspension were spotted onto IM plates containing acetosyringone, and residual mixture was taken for confocal imaging to confirm adhesion of Agrobacterium to Microbotryum cells (Figure C4-3). 


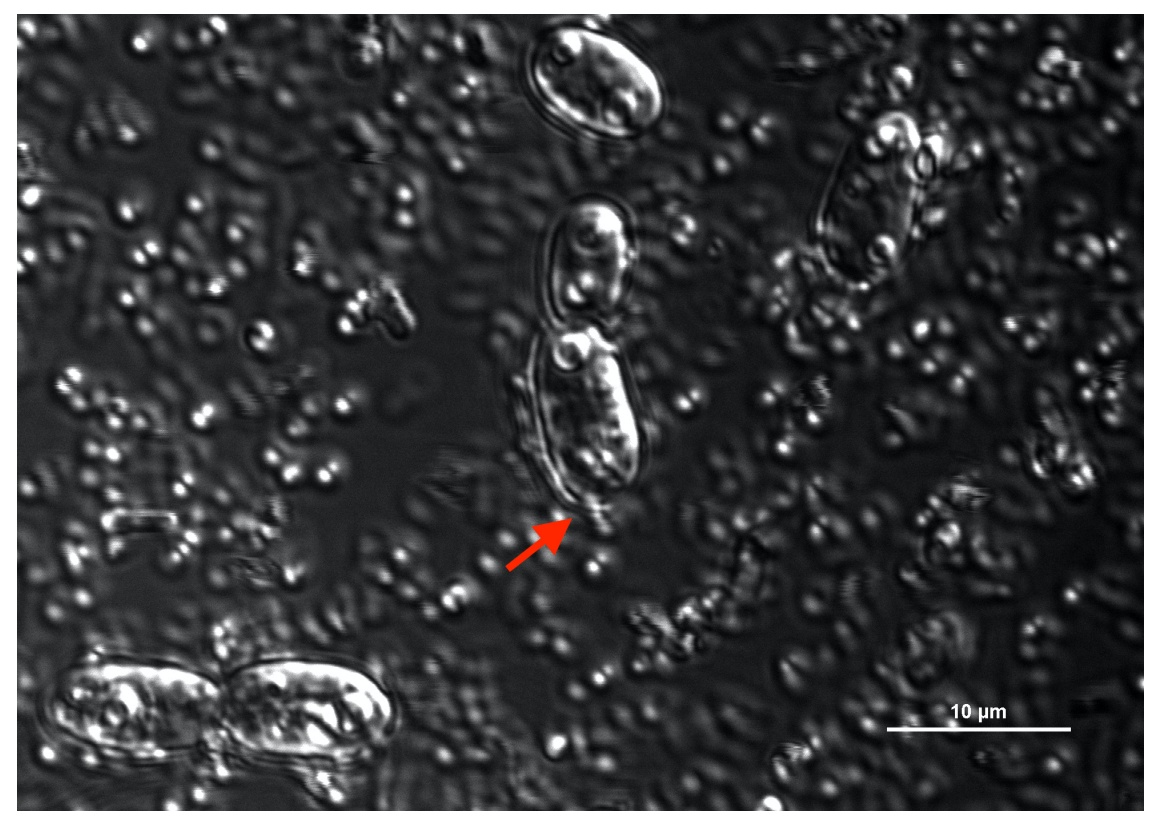

Figure C4-3 Confocal image of Agrobacterium-mediated transformation of Microbotryum cells.

Microbotryum cells appear as large oval structures approximately 10 microns in length. Attached agrobacterium cells are about 1 micron and labeled by red arrow.

Spotted plates were incubated at room temperature, $\sim 25^{\circ} \mathrm{C}$, for 3 days, after which the resulting mass of cells was scraped from the plates, suspended in $600 \mu \mathrm{L}$ of YPD-10\% broth, and $200 \mu \mathrm{L}$ of each suspension were spread onto YPD-10\% containing $150 \mu \mathrm{g} / \mathrm{mL}$ Hygromycin and $100 \mu \mathrm{g} / \mathrm{mL}$ Cefotaxime plates. Each plate was then incubated for 12-15 days to select for transformed Microbotryum cells. Colonies were streaked onto fresh YPD-10\% containing $150 \mu \mathrm{g} / \mathrm{mL}$ Hygromycin B plates for immediate storage. Putative transformants verified for successful species-specific gene insertions via PCR amplification, including the hyg gene and unique targets of each construct; DNA sequencing of amplified regions ensured the appropriate insertions/locations. Such verified transformants were then used for cross-infection studies in the respective host plant species.

\subsection{Cross-infection analysis for M. lychnidis-dioicae expressing MvSd_09295}

Cross-infection studies for the MvSd_09295 gene were performed in Bochum, DE using the growth facilities and electron microscope available at the Ruhr-Universität Bochum. a1 and a2 mating types for M. lychnidisdioicae (p1A1 and p1A2 strains; Perlin et al., 2015; Toh et al., 2017), M. silenes-dioicae, and the M. lychnidis- 
dioicae expressing the MvSd_09295 transgene were grown of YPD-10\% plates, with added $150 \mu \mathrm{g} / \mathrm{mL}$ Hygromycin B for the transgenic strain, for $48 \mathrm{~h}$ before suspension in water at an $\mathrm{OD}_{600 \mathrm{~nm}}$ of 1 . The corresponding mating types were then mixed at equal volumes and $10 \mu \mathrm{L}$ of the cell suspensions were spotted onto S. latifolia and S. dioica seeds, totaling in 6 groups (Figure C4-4). For each group, 100 seeds for each group (300 S. latifolia and 300 S. dioica seeds in total) were evenly spaced on 2 large Minimal Salt (MS) $1.8 \%$ agar plates, 50 seeds on each plate. Each seed was spotted with $10 \mu \mathrm{L}$ of cell suspension containing both mating types of the Microbotryum for that particular Microbotyrum/Silene grouping. The plates of spotted seeds were then sealed using parafilm and placed in the dark for $48 \mathrm{~h}$ at $4{ }^{\circ} \mathrm{C}$ for vernalization and to allow for fungal mating to occur. The plates were then moved to a growth chamber with the following program: On - 4am, $16 \mathrm{~h}$ day length, $22{ }^{\circ} \mathrm{C}$ day, $18{ }^{\circ} \mathrm{C}$ night, $136 \mathrm{mmol} / \mathrm{m}^{2} / \mathrm{sec}$, fluorescent bulbs, $50-60 \%$ humidity.

Seeds were spotted in the chamber with sterile water every other day for two weeks. After 11 days, at least one sprouted seedling was taken from each of the 6 groups and observed via electron microscopy. Seedlings (defined in this study as sprouting seeds with cotyledon leaves) and sprouts (defined as sprouting seeds with only the emerging root tip) from each plate were transplanted into pots filled with dampened soil based on their overall growth progression, 11 days for Silene latifolia and 14 days for Silene dioica (Figure C4-4-A). Individuals from each group were transplanted, selecting first all of the seedlings followed by the sprouts to bring the total number for each group to 80 . The number of seedlings and sprouts for each group is listed in Figure C4-4-A. After transplanting, Hypoaspis miles eggs, purchased from Sautter \& Stepper, were sprinkled onto the top of the pots to act as a biological pest control. The pots were then arranged in randomized blocks, each block containing one pot for each of the 6 groups (Figure C4-4-B). Four blocks each were then randomly placed into 20 total watering trays (Figure C4-4-C) and moved to the greenhouse (Figure C4-4-D). 
A.

Microbotryum Strain

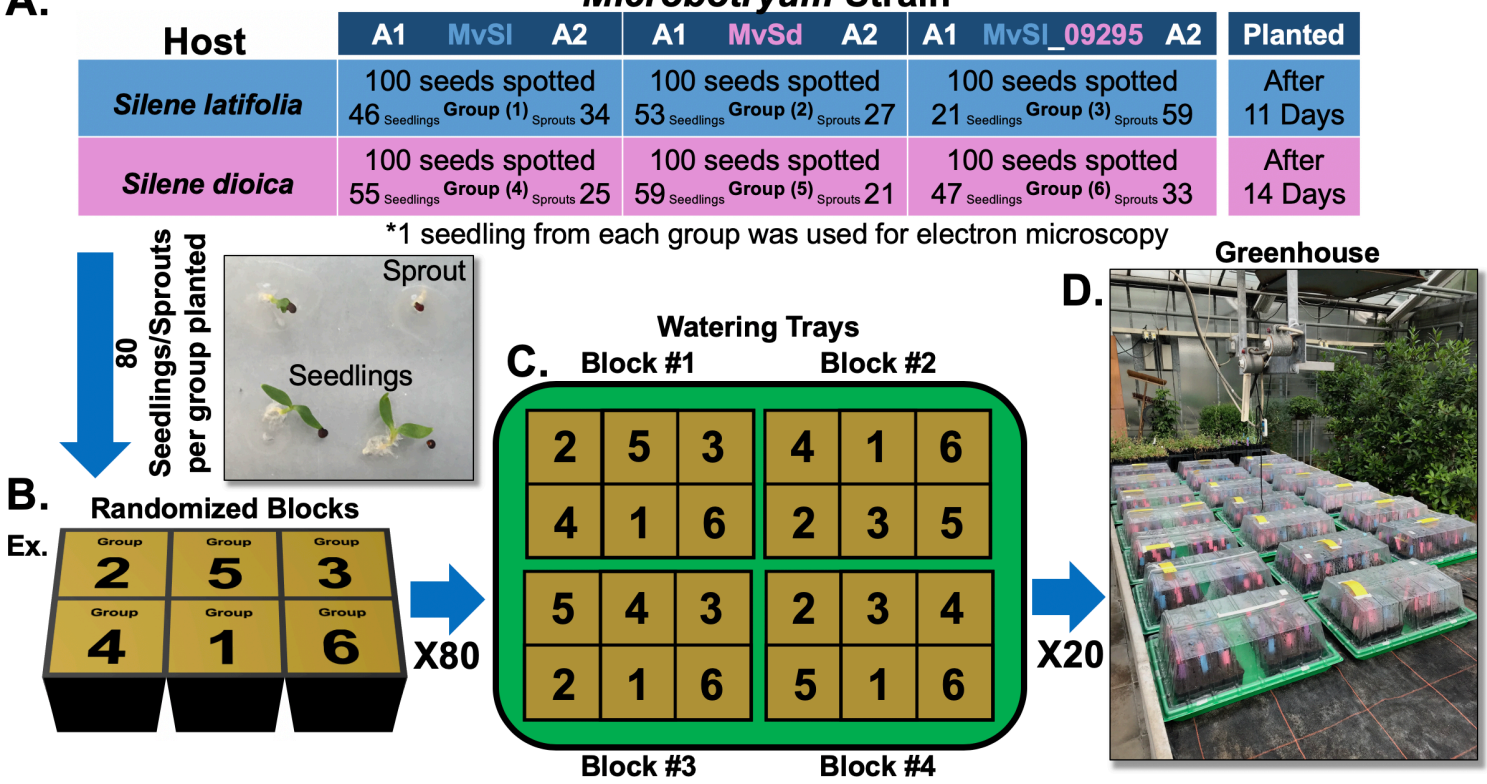

Figure C4-4 Outline of the planting procedures used in the Ruhr-Universität Bochum study. (A)

demonstrates the total number of seeds spotted with a1 and a2 mating types of each Microbotyrum strain, as well as the number of seedlings and sprouts that were transplanted into soil after an incubation period (planted column). Each cell also contains the metadata number for each Microbotryum/Silene grouping. Seeded pots were then randomly organized in blocks of 6 (B), with one representative from each group in each block. Groups of four blocks were then randomly placed into 20 watering trays (C) and moved to the greenhouse (D).

The arrangement of each pot is illustrated in Figure C4-5. A lid was added to each tray to trap moisture from evaporation, and an insect strip was placed on the top of each tray near the opening to help trap insects (Figure C4-2-D). The lids for each tray were removed after 1 month of growth as the plant became tall enough to reach the lid. The experiment was run for a total of 1 year, with Silene latifolia hosts flowering after 2-3 months and the Silene dioicae hosts requiring overwintering before flowering between 10-12 months. Windows in the green house remained open for the duration of the experiment to allow for ambient temperatures, which were recorded and are listed in Figure C4-5. 

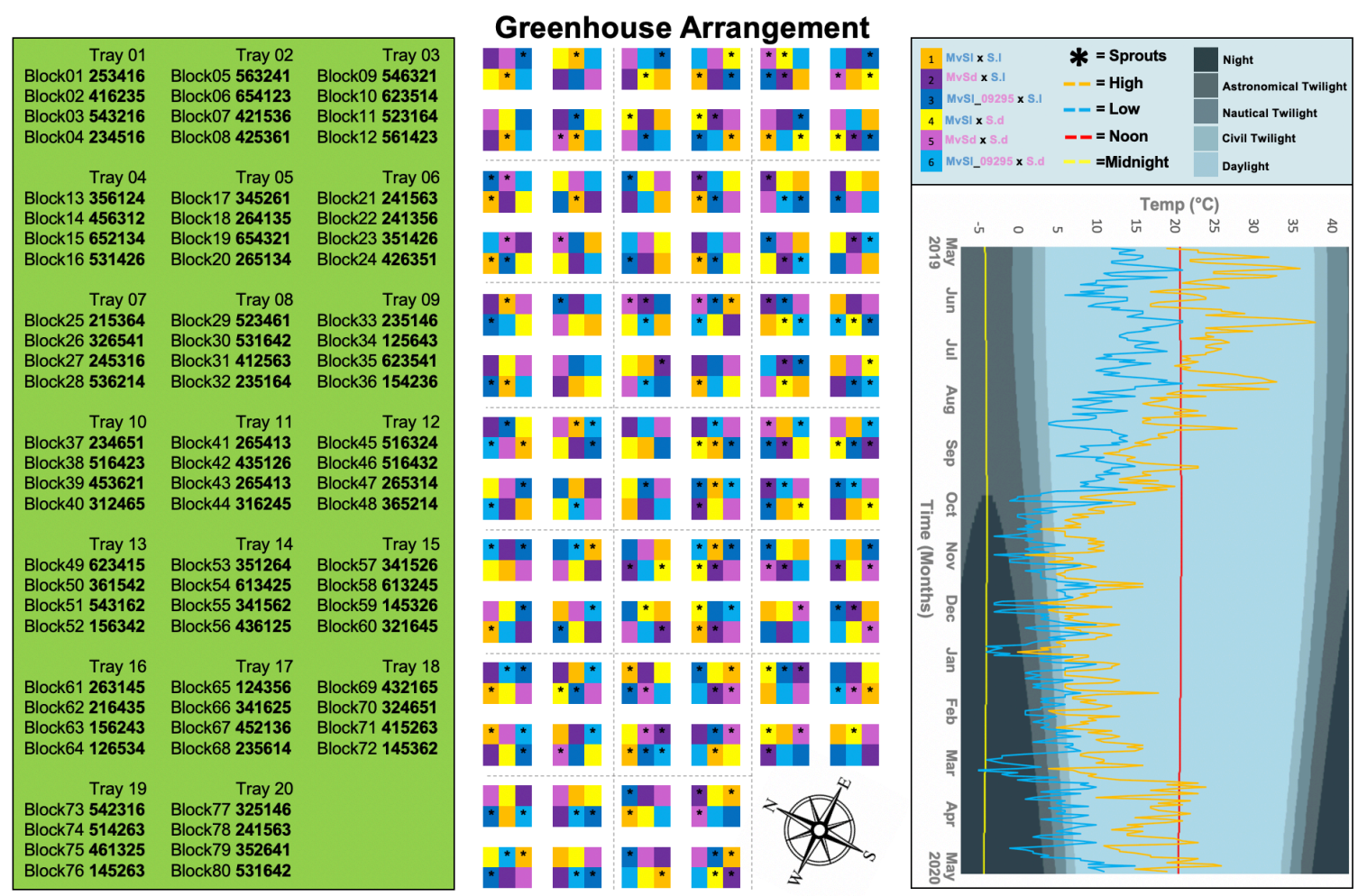

Figure C4-5 Greenhouse arrangement of plants in the Bochum study. demonstrates the arrangement for

the randomized blocks and the conditions in the greenhouse. The order of groups for each block, and the order of blocks in each tray, was randomized using the List Randomizer tool at Random.org, results of which are shown in the green left column. Trays were organized in lines of three to fit the bench top of the greenhouse laying from NE to SW as shown in the white middle column. Each group is color coded by the plant and strain used, 1 (orange) represents MvSl on S. latifolia, 2 (yellow) represents MvSd on S. latifolia, 3 (light green) represents the transgenic MvSl expressing the MvSd_09295 gene on S. latifolia, 4 (dark green) represents MvSl on S. dioica, 5 (light blue) represents MvSd on S. dioica, and 6 (dark blue) represents the transgenic MvSl expressing the MvSd_09295 gene on S. dioica. Solid color squares represent pots that contained transplanted seedlings, while squares including a asterisk $\left(^{*}\right)$ symbol represent pots that contained transplanted sprouts. The daylight cycle for the greenhouse, divided into periods of international Night, Astronomical Twilight, Nautical Twilight, Civil Twilight, and Daylight, are displayed in the right column. Noon and midnight are represented by the red line and yellow line, respectively. The high (orange) and low (blue) temperature for each day throughout the year of the trial are superimposed over the daylight cycle. 


\subsection{Electron microscopy for MvSd_09295}

SEM was performed on 1 seedling for each group with the exception of the MvSl control strain on S. latifolia grouping, which used 3 seedlings due to an inability to find dikaryotic filaments. Samples were manually opened, mounted on brass blocks with a mixture of Tissue Tek O.C.M. compound and colloidal graphite, and directly fixed using slushy nitrogen freezing and a cryo-transfer system (Quorum PT3000T) to prevent artifacts of chemical fixation and freeze drying. Once in the cryo-transfer system, water was sublimed at $105^{\circ} \mathrm{C}$ for $20 \mathrm{~min}$, the specimens were sputtered with platinum at $30 \mathrm{~mA}$ for $90 \mathrm{sec}$ and analyzed at $-140^{\circ} \mathrm{C}$ at high vacuum with the SE2 detector using a Sigma VP at $8 \mathrm{kV}$ and an aperture of $30 \mu \mathrm{m}$.

\subsection{Cross-infection analysis for MvS1_01693 in M. silenes-dioicae}

Cross-infection studies for the MvSl_01693 gene were performed in Kentucky, US using the University of Louisville growth facilities. a1 and a2 mating types for M. silenes-dioicae and the M. silenes-dioicae expressing the MvSl_01693 transgene were grown on YPD-10\% plates, with added $150 \mu \mathrm{g} / \mathrm{mL}$ Hygromycin $\mathrm{B}$ for the transgenic strain, for $48 \mathrm{~h}$ before suspension in water at an $\mathrm{OD}_{600 \mathrm{~nm}}=1.0$. The corresponding mating types were then mixed at equal volumes and seeds were spotted and cooled for vernalization and fungal mating in similar fashion to the study performed in Bochum, DE. The plates were then moved to a growth chamber with the following program to simulate the temperature and day cycle conditions in Bochum, DE during the beginning of flowering season for Microbotryum (May) with a reduction of 1 hour to conform with pervious growth experiments demonstrated to generate successfully infected hosts (Toh \& Perlin, 2015): Dawn from 8:00-9:00 $16^{\circ} \mathrm{C} 8 \mathrm{x}$ incandescent bulbs, Day from 9:00-21:00 $20^{\circ} \mathrm{C} 8 \mathrm{x}$ incandescent bulbs with light intensity $136 \mu \mathrm{mol} / \mathrm{m}^{2} / \mathrm{sec}$ at plant surface, Dusk from 21:00-22:00 $20^{\circ} \mathrm{C} 8 \mathrm{x}$ incandescent bulbs, Night from 22:00-9:00 $16^{\circ} \mathrm{C}$ no blubs on (Figure $\left.\mathrm{C} 4-6\right)$. 


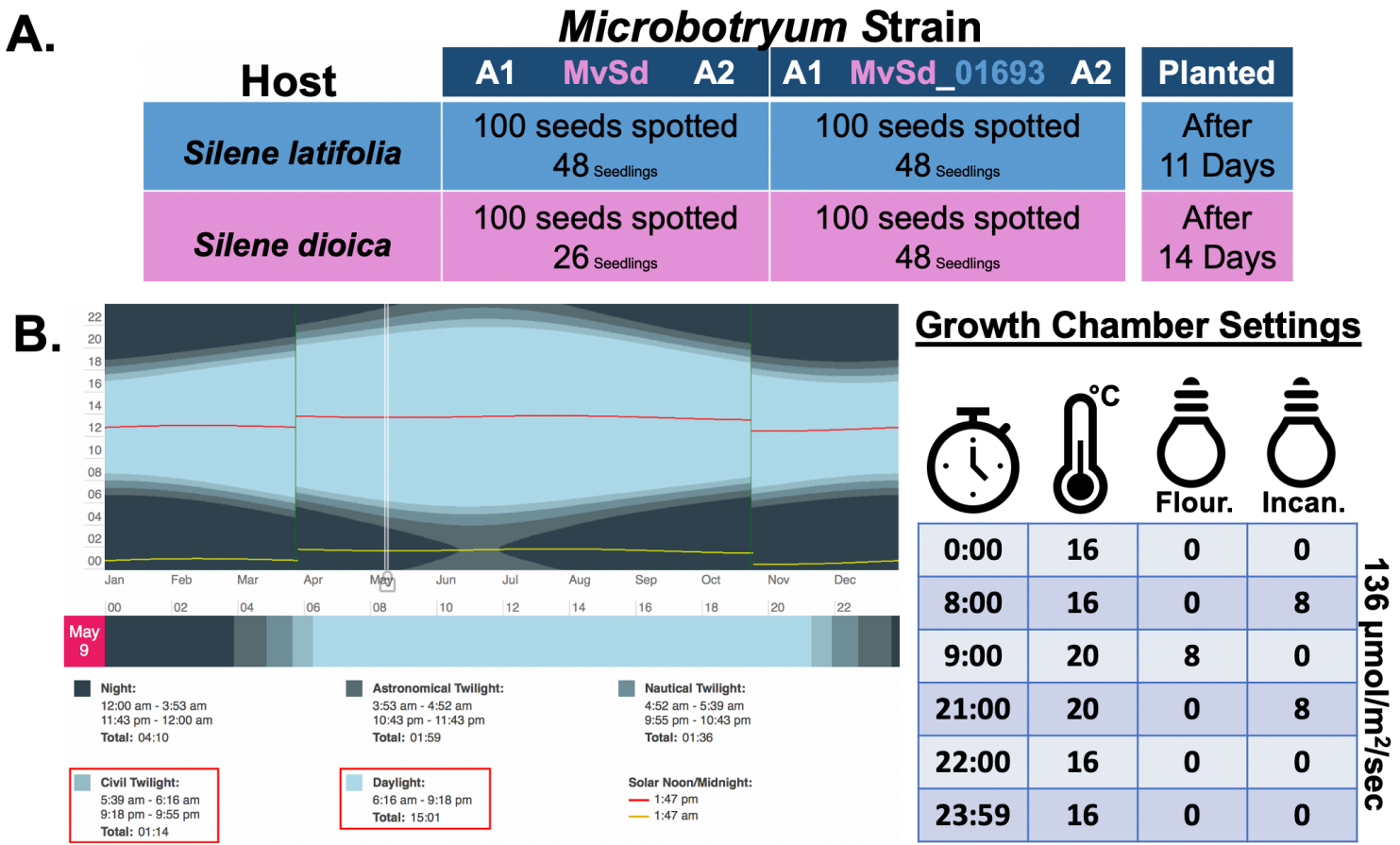

Figure C4-6 Growth chamber conditions for the Louisville study. A) Breakdown of the four groups tested in the Louisville experiment by number of seeds spotted, number of seedlings transplanted, and after number of days of growth on water agar plates. B) Light cycle graph for Bochum, DE in May from Timanddate.com, left, and growth chamber conditions used for the Louisville experiment, right.

Because very few infected sprouts used in the Ruhr-Universität Bochum study survived to adult plants for analysis, only seedlings were planted for the Louisville study. S. latifolia seedlings were again transplanted after 11 days and $S$. dioica were transplanted after 14 days in similar fashion with the Bochum study. Without access to electron microscopy, dikaryotic filaments could not be observed; however, mating of the fungal strains were verified via light microscopy. Groups were again randomized into blocks; however, the number of blocks was limited to 40 for the Louisville study due to growth chamber size restrictions. Seedlings were transplanted into eggshell crates filled with dampened Star-Green Potting Mix Plus Fertilizer soil containing $0.1 \%$ Nitrogen, $0.08 \%$ Phosphate, and $0.06 \%$ Soluble Potash. Eggshell crates were fitted into trays to water the seedlings from the bottom up. After the seedlings reached the 8-leaf stage, they were transplanted into their own small pot with the same dampened Star-Green Potting Mix and placed in a tray to water from the bottom up. 


\section{Results}

\subsection{Microscopy results}

With the access to electron microscopy in the Bochum study, we were able to verify the formation of a dikaryotic filament and penetration of the plant cell in all but the MvSl on S. latifolia control group (Figure C4-7). For each successful attempt, only one seedling was needed, indicating that successful infection was highly probable amongst the different groups. To rule out the chance that a lack of infection seen in the MvSl $\mathrm{x}$ S. latifolia pairing may have been due to random selection of non-infected seedlings, three seedlings were observed, all of which lacked the presence of dikaryotic filaments (Figure C4-7).

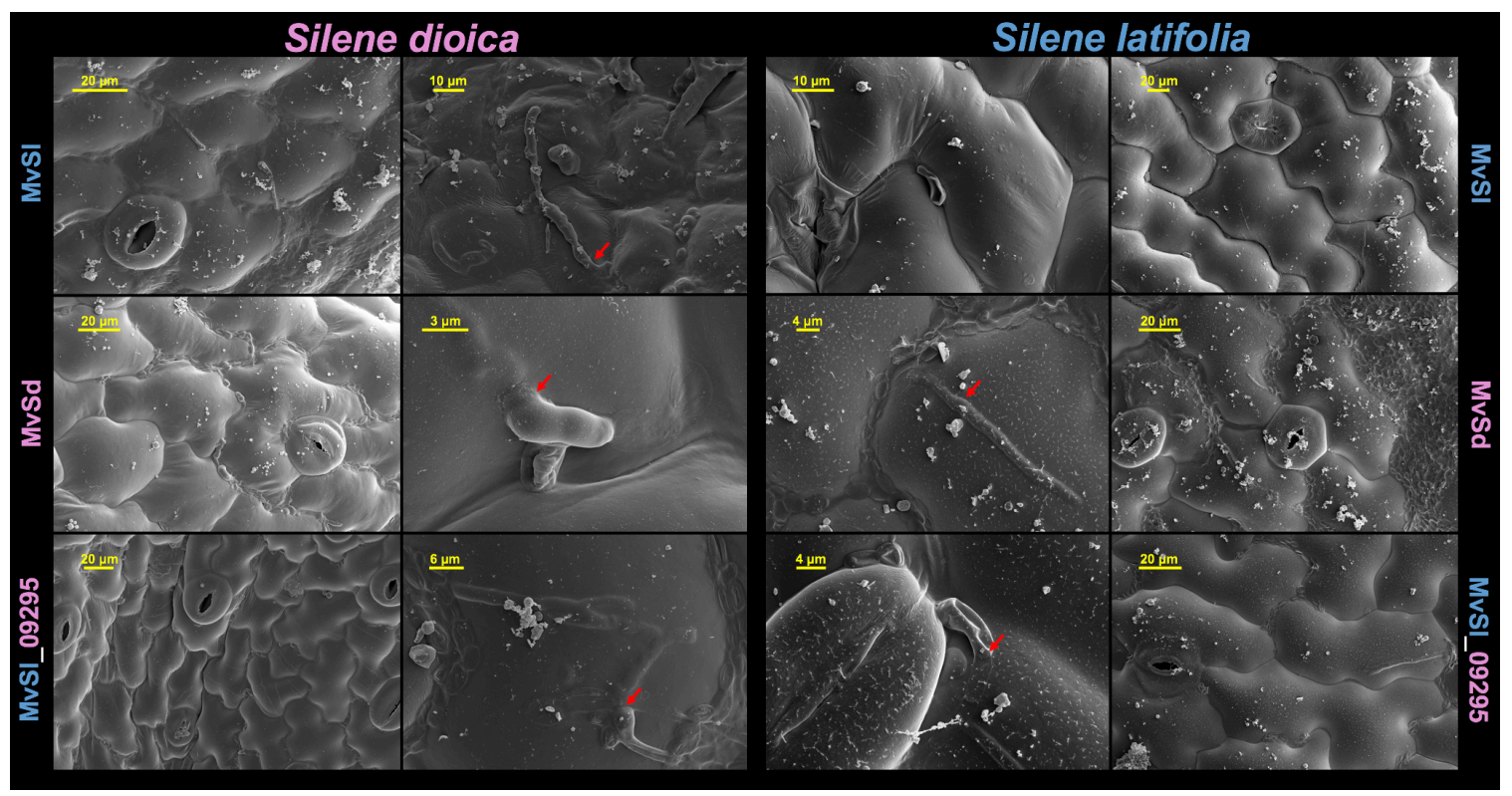

Figure C4-7 Electron micrographs of seedlings infected with Microbotryum. The images were taken of the cotyledon leaves of one seedling for each of the 6 groups, with the exception of MvSl on Silene latifolia for which three seedlings were observed. An overview is shown on the left of each paring, while a closeup of appressorium are shown on the right. The left column are images of infected Silene dioica seedlings, while the right column shows infected Silene latifolia seedlings. Each row indicates the strain/species of Microbotryum that was used for infection, $\mathrm{MvSl}$ (top), MvSd (middle), and transgenic MvSl expressing the MvSd_09295 gene (bottom). 
To further rule out a lack of mating as the cause for a lack of dikaryotic filament formation in the MvSl x $S$. latifolia control group, samples were taken from the agar surrounding the spotted seedlings and mating was verified (Figure C4-8). These images demonstrate the MvSl strains were able to mate, forming conjugation tubes; however, they did not infect the seedlings on which they were spotted.

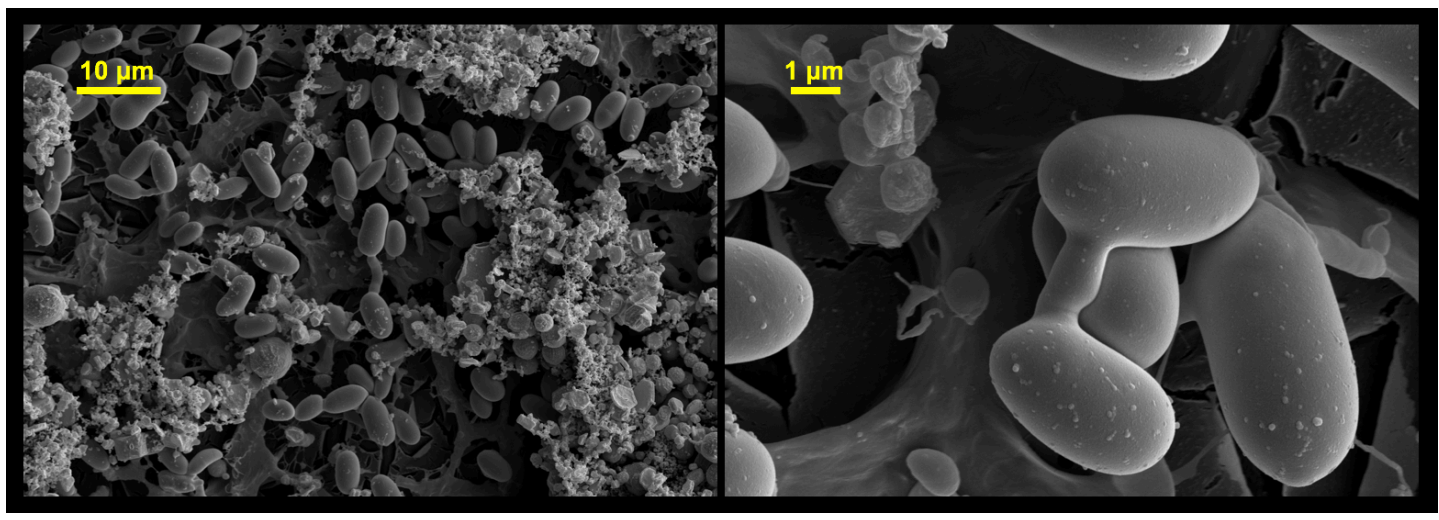

Figure C4-8 Electron micrographs of mating MvSl a1 and a2 cells. Cells were taken from MS agar around the locations where seedlings were removed.

While we were unable to observe dikaryotic filament formation on seedlings without electron microscopy in the Louisville study, mating was verified using light microscopy from cell suspensions located around spotted seeds (Figure C4-9).

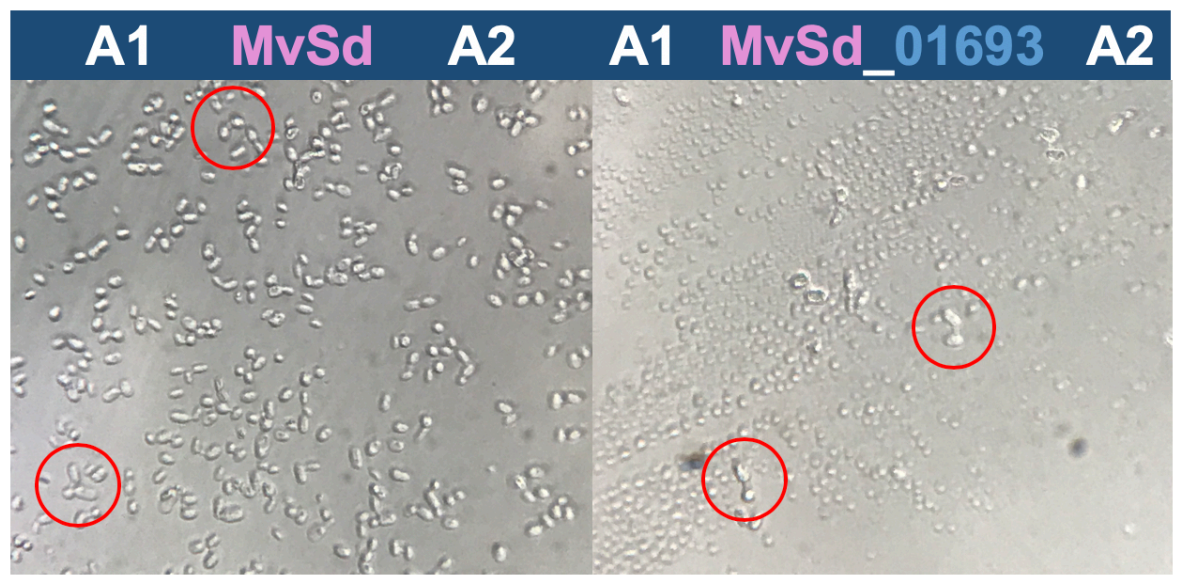

Figure C4-9 Confirmation of Microbotryum mating via light microscopy. Mating was observed between a1 and a 2 mating types of the $M$. silenes-dioicae control (MvSd), left, and a1 and a 2 mating types of the $M$. silenes-dioicae strain expressing the M. lychnidis-dioicae transgene MvS1_01693 (MvSd_01693), right. 
Observation of conjugation tube formation in Figure C4-9 demonstrates that the proper mating types for each strain were mixed, and while they do not demonstrate the formation of the infectious dikaryotic filament the eventual observation of infected adult plants for each group corroborate successful mating an infection.

3.2 Infection results for the MvSd_09295 Ruhr-Universität Bochum study

From the six groups in this study, MvSl x S. latifolia, MvSd x S. latifolia, MvS1_09295 x S. latifolia, MvS1 x S. dioica, MvSd x S. dioica, and MvS1_09295 x S. dioica, all three groups of S. latifolia plants flowered between 2-3 months. All 3 S. dioica groups required overwintering in the greenhouse and flowered between 13-14 months. Of the individuals planted as sprouts, only $10.55 \%$ survived into adult plants. This was compared to an overall survival rate of $74.73 \%$ for individuals transplanted as seedlings. For the groups infecting S. latiofolia hosts, $100 \%$ of the 93 adults survived to flowering; however, no infected flowers were observed (Table C4-3). Similarly, 100\% of the 139 flowers that survived to adult plants flowered in the groups infecting $S$. dioica hosts, but only one infected host was observed in each group (Table C4-3).

Table C4-3. Tally of seedlings surviving, flowering, and infected flowers for the Bochum study

\begin{tabular}{|c|c|c|c|c|c|c|}
\hline Spotted & Transplanted & Adult Plants & Flowered & Infected & 1 & MvSI x S.I \\
\hline \multirow{2}{*}{100 Seeds } & 46 Seedlings & 35 (76.09\%) & \multirow{2}{*}{$43(100 \%)$} & \multirow{2}{*}{$0(0 \%)$} & 2 & MvSd x S.I \\
\hline & 34 Sprouts & $8(25.23 \%)$ & & & 3 & MvSI_09295 x S.I \\
\hline \multirow{2}{*}{100 Seeds } & 53 Seedlings & $26(49.06 \%)$ & \multirow{2}{*}{$30(100 \%)$} & \multirow{2}{*}{$0(0 \%)$} & 4 & MvSI x S.d \\
\hline & 27 Sprouts & $4(14.81 \%)$ & & & 5 & MvSd $\mathbf{x}$ S.d \\
\hline \multirow{2}{*}{100 Seeds } & 21 Seedlings & $15(71.43 \%)$ & \multirow{2}{*}{$21(100 \%)$} & \multirow{2}{*}{$0(0 \%)$} & 6 & MvSI_09295 x S.d \\
\hline & 59 Sprouts & $6(10.17 \%)$ & & & & \\
\hline \multirow{2}{*}{100 Seeds } & 55 Seedlings & $43(78.18 \%)$ & \multirow{2}{*}{$45(100 \%)$} & \multirow{2}{*}{$1(2.22 \%)$} & & \\
\hline & 25 Sprouts & $2(8.00 \%)$ & & & & \\
\hline \multirow{2}{*}{100 Seeds } & 59 Seedlings & 55 (93.22\%) & \multirow{2}{*}{$55(100 \%)$} & \multirow{2}{*}{$1(1.81 \%)$} & & \\
\hline & 21 Sprouts & $0(0 \%)$ & & & & \\
\hline \multirow{2}{*}{100 Seeds } & 47 Seedlings & $36(76.60 \%)$ & \multirow{2}{*}{$39(100 \%)$} & \multirow{2}{*}{$1(2.56 \%)$} & & \\
\hline & 33 Sprouts & $3(9.09 \%)$ & & & & \\
\hline
\end{tabular}


3.3 Infection results for the MvS1_01693 University of Louisville study

From the four groups in this study, MvSd_01693 x S. dioica, MvSd x S. dioica, MvSd_01693 x S. latifolia, and MvSd x S. latifolia, all transplanted seedlings survived to adult plants; however, only S. dioica hosts induced flowering (Table C4-4). Unlike in the MvS1_09295 study, the first flowers observed were from the MvSd_01693 x S. dioica group, only 56 days post transplantation of seedlings into soil. This was compared to 67 days for the first flowers of the control group, MvSd x S. dioica (Table C4-4). In total, nearly half (46.51\%) of the flowers from the MvSd_01693 x S. dioica group were infected compared to only $16.66 \%$ in the control MvSd x S. dioica group (Table C4-4).

Table C4-4. Tally of seedlings surviving, bolting, flowering, and infected flowers for the Louisville study

\begin{tabular}{|c|c|c|c|c|c|c|}
\hline Spotted & Transplanted & Adult Plants & Flowered & Infected & 1 & MvSd_01693 x S.I \\
\hline 100 Seeds & 48 Seedlings & $48(100 \%)$ & $0(0 \%)$ & N/A & 2 & MvSd x S.I \\
\hline 100 Seeds & 26 Seedlings & $25(100 \%)$ & $0(0 \%)$ & N/A & 3 & MvSd_01693 x S.d \\
\hline 100 Seeds & 58 Seedlings & $48(100 \%)$ & $43(89.58 \%)$ & $20(46.51 \%)$ & 4 & MvSd x S.d \\
\hline 100 Seeds & 48 Seedlings & $48(100 \%)$ & $18(72.00 \%)$ & $3(16.66 \%)$ & & \\
\hline
\end{tabular}

While both male and female flowers were observed infected in the MvSd_01693 x S. dioica group at a nearly 1:1 ratio (11 females and 9 males), all three infected flowers observed in the control MvSd x S. dioica group were male flowers. The infected female flowers observed in the MvSd_01693 x S. dioica group had induced pseudo-anther production in the floral ovary at the base of the flower (Figure C4-10-B). 

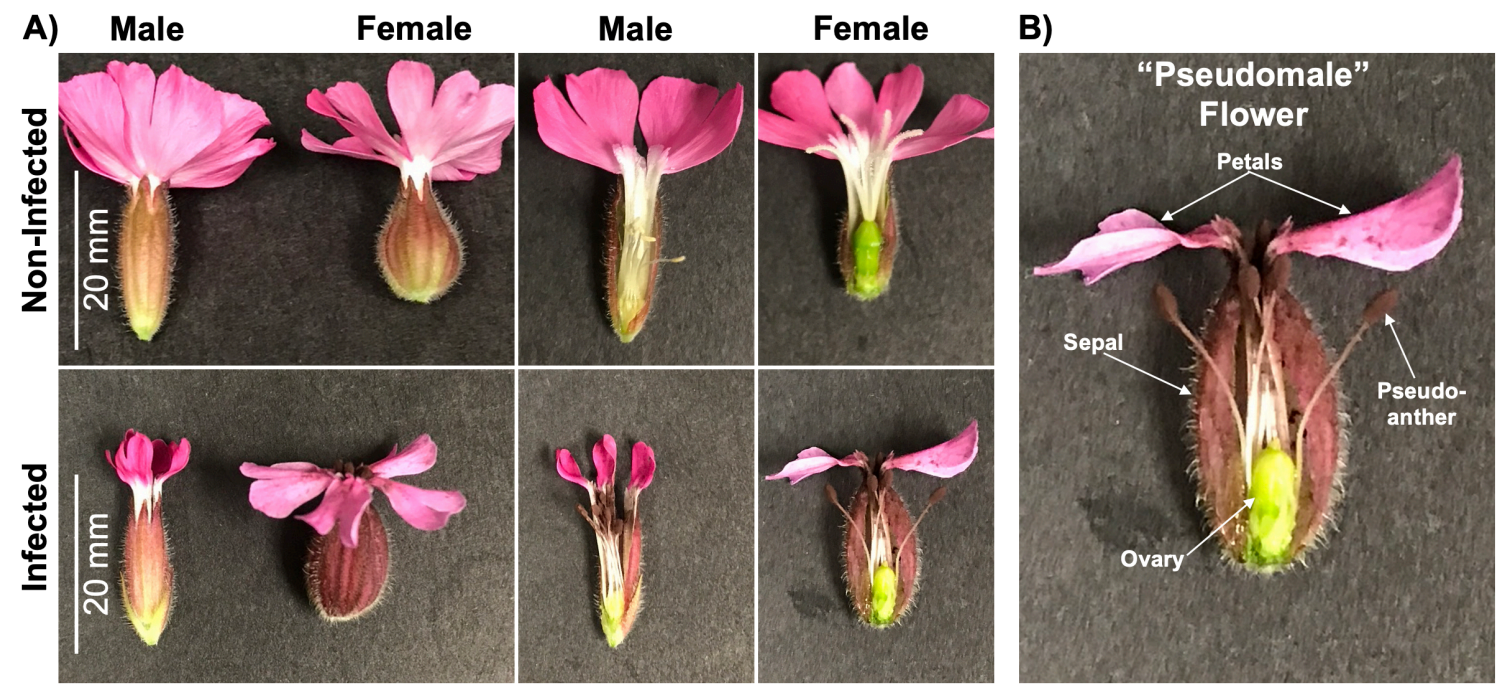

Figure C4-10 Infected and non-infected Silene dioicae flowers. Non-infected male and female flowers are shown in the top row of (A), while infected male flowers and infected female flowers with induced pseudoanthers are shown in the bottom row of (A). B) demonstrates a closeup image, labeled with the morphological changes induced by infection of a female $S$. dioica flower, referred to as a "pseudomale" flower.

\section{Discussion}

This study examines the effect of species-specific genes on host-specificity in the Microbotryum genus through heterologous expression of genes unique to each of the sister species, M. lychnidis-dioicae and $M$. silenes-dioicae. The results for the two genes analyzed, MvSd_09295 and MvS1_01693, were mixed, with MvSd_09295 providing no difference towards infection on either host when expressed in M. lychnidisdioicae and MvSl_01693 demonstrating a significantly increased capacity for infection of S. dioica when expressed in M. silenes-dioicae. While these results indicate that some species-specific genes may play a role in modulation of the host defense response, differences between the experimental setups may have contributed to observed differences in results, and therefore these studies must be repeated for further support of our hypotheses.

One major difference observed between the Bochum study and the Louisville study was the growth rate of $S$. dioica hosts. In the Bochum study, which used ambient greenhouse condition in Bochum, DE, $S$. dioica plants took over a year to flower (14 months). This dramatically differed from the $S$. dioica plants grown in the growth chambers in the Louisville study, which were able to flower after just 56 days. One 
contributing factor may have been the growth chamber conditions used in Louisville, which mirrored the daylength and light intensity found during the flowering season for $S$. dioica, typically around May. Given that day length is a known major factor that contributes to signaling of flower production in plants, suspending plant growth under light conditions consistent with the flowering period, as opposed to our study in Bochum for which plants did not reach their adult stage until July, may explain the differences observed in flower timing. These results suggest that further studies performed in the greenhouse environment may be able to save time if started earlier in the year by aiming to have a majority of plants reach their adult stage in April. Alternatively, this paper highlights optimal conditions for growth of S. dioica plants in a chamber environment, which may prove to be a much more effective approach.

In addition to differences observed between studies, there is evidence to support that the control group MvSl x S. latifolia in the Bochum study may have been uninfected due to lab errors as well. This is shown by the lack of observable dikaryotic filament structure formation in the control group on all three seedlings observed, but clear dikaryotic filament formation in the two test groups for the S. latifolia host. One possible explanation for the lack of formation of dikaryotic infective structures in mated a1 and a2 strains of the MvSl control observed in the Bochum study may be due mislabeling of strains in the lab. While the MvSl strains transformed with the MvSd_09295 gene were generated in the lab at the University of Louisville, the control strains were taken from storage at Ruhr-Universität Bochum. Pervious work has demonstrated that post-zygotic barriers exist in hybridized strains of Microbotryum, which may explain why dikaryotic filament formation was not observed between if the matting assay was mistakenly set up as a mating event between two different species of Microbotryum. This may also explain why mating was still observed in the areas around where the seedlings were extracted from the agar.

While some species-specific SPs appear to play a role in pathogenicity of in their progenitor species, it is evident from this study that not all species-specific genes result in unlocking pathogenesis of their corresponding hosts. In fact, while a significant increase in ability for M. silenes-dioicae to infect its own host, S. dioicae, was observed in the transgenic strains expressing the MvSl_01693 gene, a greater ability for the transgenic strain to infect the host associated with the MvS1_01693 SP, S. latifolia, was not observed. Furthermore, the MvSd_09295 species-specific SP did not demonstrate a change in pathogenicity in either direction on either host. Despite these findings, the results from the heterologous expression of MvS1_01693 
in M. silenes-dioicae are of interest. Increased ability of M. silenes-dioicae to infect its natural hosts, S. dioica, is outside of our original hypothesis which stated that species-specific genes were important for infection of their own associated hosts (i.e., an M. lychnidis-dioicae species-specific SP should have conferred a greater ability to infect the natural M. lychnidis-dioicae host, S. latifolia), but could help explain the genesis of such species-specific genes. One possible explanation for the increased pathogenicity observed in the transgenic MvSd_01693 strain on its own host, S. dioica, may be that the MvSl_01693 gene evolved initially in $M$. lychnidis-dioicae in response to coevolutionary pressures arising from changes in S. latifolia after the speciation event separating the two sister species/host pairings. If the novel MvS1_01693 gene coded for a SP that conferred a significant initial advantage for the pathogen during host pathogenicity, this gene would have spread rapidly through the M. lychnidis-dioicae population. Over time however, resistance to the effects of the MvS1_01693 SP would have been selected for in the S. latifolia population as a consequence of rapid spreading of this new strain of M. lychnidis-dioicae, resulting in recognition factors or other defense responses to the MvS1_01693 SP. This would explain why when the MvSl_01693 gene is introduced to $M$. silenes-dioicae/S. dioicae pathogen/host group, an SP for which the fungi does not otherwise express naturally, the host is more susceptible having not developed an immune response over years of reciprocal selective pressure. Therefore, we may expect that species-specific genes have arisen as a direct result of the selective pressures issued by the coevolutionary struggle between pathogen and host over time post speciation events, and not through a gene loss mechanisms in the other species. Furthermore, the demonstrated efficacy of species-specific SPs in alternative hosts seen in this study could help facilitate host shifts events withing the Microbotryum genus.

Understanding how these species-specific genes arise and the role they play in host-specialization sheds light on how coevolutionary pressures can change pathogens over time. While we were able to observe increase pathogenicity in the transgenic MvSd strains when infecting their own hosts, it is likely that modifications to core secreted proteins play a much larger role in coevolution and subsequent speciation between the two now separate species than unique proteins as outlined by Beckerson et al., 2019 and Chapter 2. While certain species-specific SPs may play a role in modulation of defense responses in the host, these core secreted proteins likely play a more mechanical role for entrance into the host tissue and manipulation of the plant, leading to a more stringent selective pressure. To further understand the role of the species- 
specific SPs, future studies should analyze the importance of these species-specific genes in more distantly related pairings of Microbotryum/hosts to determine their capacity for affecting pathogenicity. Alternatively, examination of the "effectorization" of orthologues in different species that appear to have lost protein secretion ability due to the accumulation of mutations during speciation events may also help to understand the coevolutionary pressures impacting the fungi. 


\section{CHAPTER 5}

\section{IMPLEMENTATION OF CRISPR-CAS9 AS AN EFFECTIVE TARGET-SPECIFIC KNOCKOUT TOOL FOR THE MICROBOTRYUM GENUS}

\section{Introduction}

The first inkling of CRISPR dates back to the late 1980's, when Yoshizumi Ishino and his lab at Osaka University discovered unusually repetitive sequences in the genome of Escherichia coli (Ishino et al., 1987). These DNA repeats would later be called Clustered Regularly Interspaced Palindromic Repeats, or CRISPR. While Yoshizumi's discovery ultimately led to a revolution in genetic modification of organisms, its full significance wasn't appreciated until investigated more fully by Francisco Mojica, who identified a similar arrangement of repeats in a very distant microbe, the halophile, Haloferax mediterranei, at the University of Alicante in Spain. Although there was no sequence similarity between the regions seen by Ishino in E. coli and those Mojica found in $H$. mediterranei, the latter realized that the arrangement of such patterns was unlikely to have occurred by coincidence (Mojica et al., 1993; Lander, 2016). Mojica continued to investigate and characterize these repeats, and by 2000 had found similar arrangements in a variety of prokaryotes, including several bacteria associated with human disease (Mojica et al., 2000). The census of microbes containing these expanded rapidly, so that by 2002 there were at least 40 species identified as having CRISPR repeats (Jansen et al., 2002). Mojica continued to investigate the role of both the repeats and the spacers that separate them, finally publishing a proposed role for them in a prokaryotic adaptive immune system (Mojica et al., 2005).

Following its discovery, two main groups recognized the importance of CRSIPR and associated Cas enzymes in genetic engineering and biotechnology. Virginijus Siksnys investigated whether the system could be reconstituted functionally in E. coli, again a distantly-related microbe of his source organism, Streptococcus thermophiles. Meanwhile, Emmanuelle Charpentier and Jennifer Doudna had formed a collaboration that led to the use of recombinant Cas9 enzyme and the single guide RNA (sgRNA) now used extensively in CRISPR transformations. Both groups published their work within two months of each other 
(Jinek et al.,2012; Gasiunas et al., 2012); however, it wasn't until 2013 that CRISPR Cas9 was successfully adapted for use in modifying the genomes of other prokaryotic and eukaryotic cells. This innovative approach of using a prokaryotic system for genetic modification in eukaryotes was implemented by Feng Zhang and his lab at the Broad Institute of MIT and Harvard, and opened a floodgate of seemingly limitless genetic engineering applications (Cong et al., 2013; Mali et al., 2013). Since then, CRISPR Cas9 has been applied to many model systems across all 5 kingdoms and new innovative discoveries are still pushing the boundaries its application (Hovath and Barrangou, 2010; Char et al., 2017; Ochiai, 2015; Lu et al., 2017) (Table C5-1).

Table C5-1. List of Model Organisms with Established CRISPR Systems

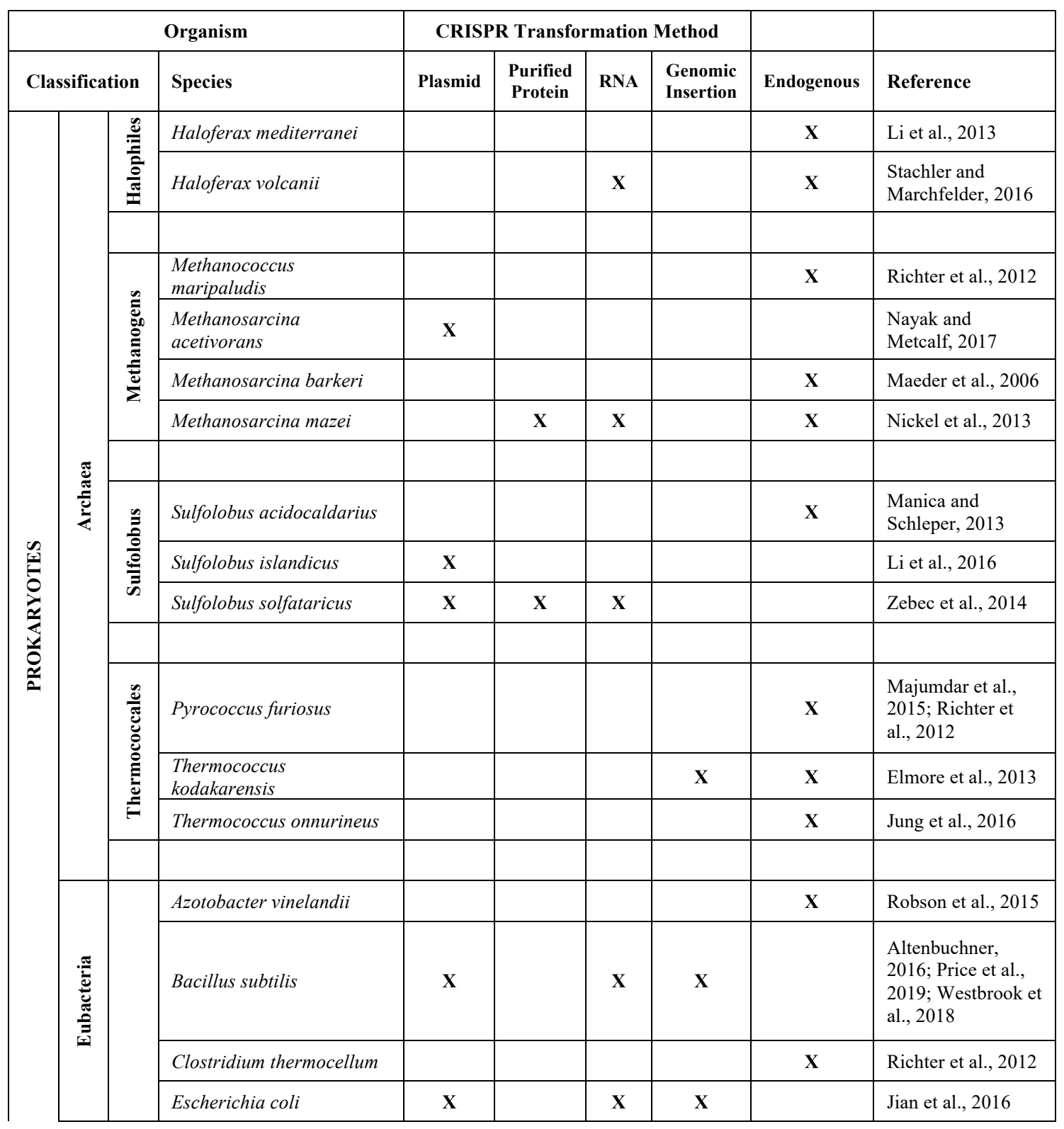




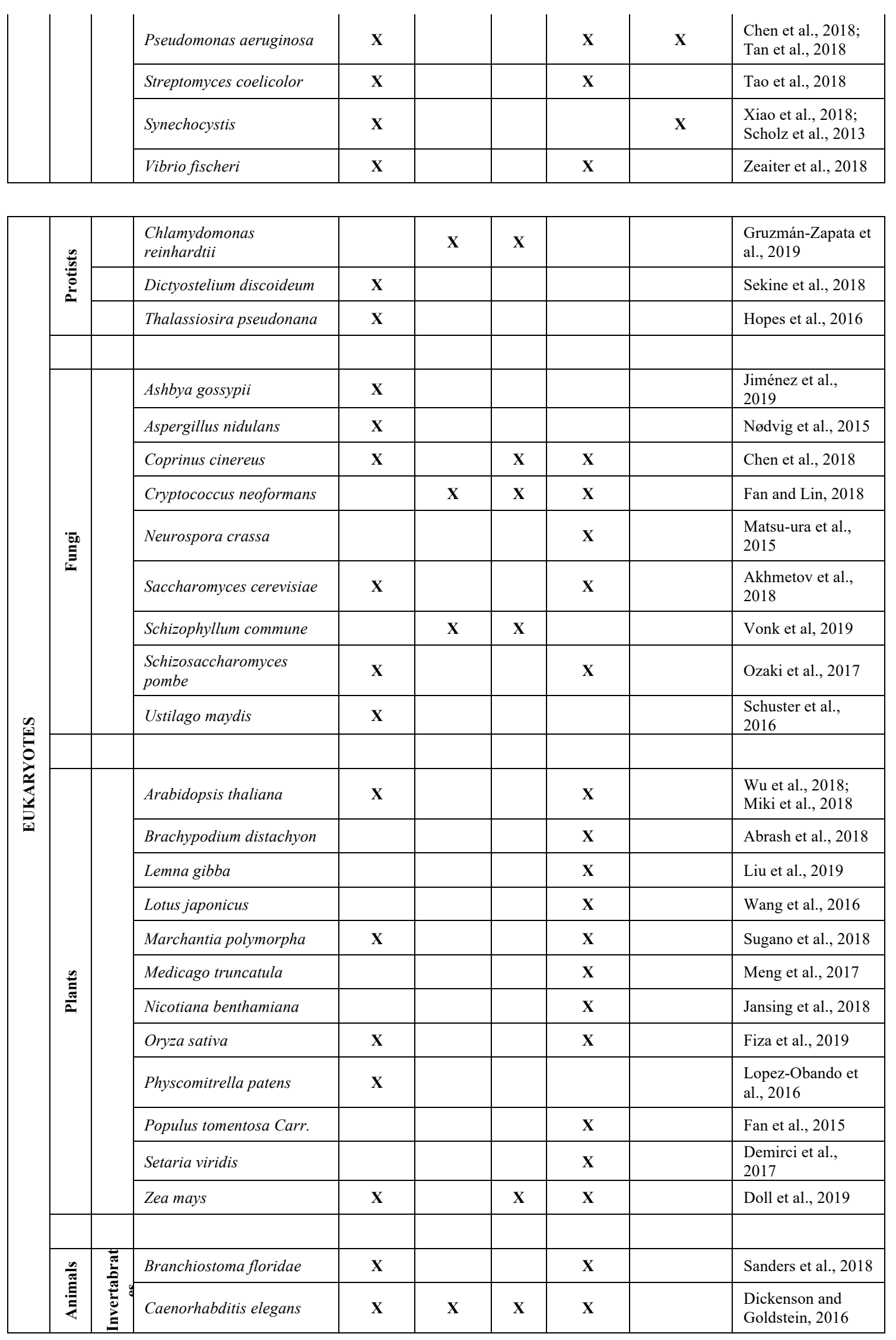




\begin{tabular}{|c|c|c|c|c|c|c|}
\hline & Coina intestinalis & $\mathbf{X}$ & & & & Stolfi et al., 2014 \\
\hline & Daphnia pulex & & $\mathbf{X}$ & $\mathbf{x}$ & & Hiruta et al., 2018 \\
\hline & Drosophila melanogaster & $\mathbf{X}$ & $\mathbf{X}$ & $\mathbf{X}$ & $\mathbf{x}$ & $\begin{array}{l}\text { Port et al., 2019; } \\
\text { Gratz et al., 2015; } \\
\text { Bier et al., 2018 }\end{array}$ \\
\hline & Galleria mellonella & & & $\mathbf{x}$ & & Wei et al., 2014 \\
\hline & Gryllus bimaculatus & & & $\mathbf{x}$ & & Sun et al., 2017 \\
\hline & Hydra & & $\mathbf{X}$ & $\mathbf{x}$ & & $\begin{array}{l}\text { Lommel et al., } \\
2017\end{array}$ \\
\hline & Mnemiopsis leidyi & & $\mathbf{X}$ & $\mathbf{X}$ & & $\begin{array}{l}\text { Presnell and } \\
\text { Browne, } 2019\end{array}$ \\
\hline & Nematostella vectensis & $\mathbf{X}$ & & $\mathbf{x}$ & $\mathbf{x}$ & Ikmi et al., 2014 \\
\hline & Oikopleura dioica & & & & & \\
\hline & Oscarella carmela & & & & & \\
\hline & Parhyale hawaiensis & & & $\mathbf{X}$ & & Fan et al., 2015 \\
\hline & Pristionchus pacificus & & $\mathbf{X}$ & $\mathbf{X}$ & & Witte et al., 2015 \\
\hline & Tribolium castaneum & $\mathbf{X}$ & & $\mathbf{X}$ & & Fan et al., 2015 \\
\hline \multirow{17}{*}{ D } & Ambystoma mexicanum & & $\mathbf{X}$ & $\mathbf{x}$ & & Fei et al., 2018 \\
\hline & Canis lupus familiaris & $\mathbf{X}$ & & & & Eun et al., 2019 \\
\hline & Carolina anole & & $\mathbf{X}$ & $\mathbf{x}$ & & Rasys et al., 2019 \\
\hline & Cavia porcellus & $\mathbf{X}$ & & & & Bierle et al., 2016 \\
\hline & Danio rerio & & $\mathbf{X}$ & $\mathbf{x}$ & & Sorlien et al., 2018 \\
\hline & Gallus gallus domesticus & $\mathbf{X}$ & & & & Zuo et al., 2016 \\
\hline & Gasterosteus aculeatus & & $\mathbf{X}$ & $\mathbf{X}$ & & $\begin{array}{l}\text { Erickson et al., } \\
2016\end{array}$ \\
\hline & Homo sapiens & $\mathbf{X}$ & $\mathbf{X}$ & $\mathbf{X}$ & & $\begin{array}{l}\text { Richardson et al., } \\
\text { 2018; Zhang et al., } \\
\text { 2017; Yang et al., } \\
2014\end{array}$ \\
\hline & Mesocricetus auratus & $\mathbf{X}$ & $\mathbf{X}$ & $\mathbf{X}$ & & Fan et al., 2014 \\
\hline & Mus musculus & & $\mathbf{X}$ & $\mathbf{x}$ & & Hirose et al., 2017 \\
\hline & Nothobranchius furzeri & & & & $\mathbf{x}$ & Harel et al., 2016 \\
\hline & Orzais latipes & & $\mathbf{X}$ & $\mathbf{X}$ & & Liu et al., 2018 \\
\hline & Petomyzon marius & & & $\mathbf{x}$ & & Square et al., 2015 \\
\hline & Rattus norvegicus & & $\mathbf{X}$ & $\mathbf{x}$ & & $\begin{array}{l}\text { Kobayashi et al., } \\
2018\end{array}$ \\
\hline & Rhesus macaque & & & $\mathbf{X}$ & & Xin et al., 2016 \\
\hline & Takifugu rubripes & & $\mathbf{X}$ & $\mathbf{x}$ & & $\begin{array}{l}\text { Kato-Unoki et al., } \\
2018\end{array}$ \\
\hline & Xenopus tropicalis & & $\mathbf{X}$ & $\mathbf{X}$ & & $\begin{array}{l}\text { Nakayama et al., } \\
2014\end{array}$ \\
\hline
\end{tabular}


CRISPR operates in nature as an adaptive immunity to viral and/or plasmid invasion of many prokaryotic species with approximately $90 \%$ of Archaea and $40 \%$ of Bacteria containing CRISPR elements in their genome (Horvath and Barrangou, 2010). Upon viral invasion, CRISPR-associated (Cas) enzymes obtain short fragments of the invading viral DNA or RNA and incorporate the genetic information into the CRISPR locus, adjacent to Cas scaffold RNA regions; when transcribed together these form the guide RNA (gRNA). These gRNAs then attach to Cas enzymes to chaperone the DNA-endonuclease complexes to the virus targets. When bound to Cas enzymes, the 5' end of the gRNA binds to approximately 20 base pairs (bp) of complementary sequence and directs a conformational change, allowing the enzyme to cut the invading viral DNA or RNA (Hsu et al., 2014). To avoid cutting its own DNA, the short complementary sequences that become integrated into the host genome for CRISPR use are spaced between repeat sequences that allow the Cas enzymes to differentiate between self and non-self sequences.

There are three types of CRISPR systems: CRISPR Types I, II, and III, that differ from one another with regards to how the CRISPR array is processed and by the number of proteins that form the Cas DNAendonucleases complex responsible for cleaving the target sequence. Each type of CRISPR system has a Cas DNA-endonuclease with a corresponding unique sequence required directly downstream of the 20 base pair target site called a Protospacer Adjacent Motif (PAM). These PAM sites vary in sequence and length by CRISPR type and by species, but are not included in the target sequence on the gRNA that chaperones the Cas endonuclease to the target site. Instead, PAM sites are located directly downstream of the target sequence and are required for activation of the DNA-endonuclease activity. Types I and III both use multi-protein complexes to recognize and cut foreign genetic material, while Type II uses a single enzyme to accomplish this task. Cas9 is one example of a single-sequence encoded Type II DNA-endonuclease. The Cas9 from Streptococcus pyogenes (SpCas9) is the predominantly used Cas9 enzyme for genetic engineering due to its short 3 bp PAM site sequence (NGG), compared to the Cas9 PAM sites for other species which have longer and less useful sequences (e.g., NNGRRT in Staphylococcus aureus and NNNNGATT in Neisseria meningitidis). For this reason, the Cas9 from S. pyogenes is simply referred to as "Cas9" in most CRISPR Cas9 work. Other Type II DNA-endonucleases, such as CRISPR-Cfp1, are smaller but share many of the same features as Cas9; however, the PAM site sequences, orientation, and target design vary. While there are certainly many interesting applications of other Type II enzymes, as well as those from Type I and III systems, 
the simplicity, short PAM site, and comparatively small coding sequence for the Cas DNA-endonuclease of the Type II system makes Cas9 the model candidate for delivery of CRISPR for gene editing in new systems.

To utilize Type II CRISPR technology for genetic engineering, there are two main components that are required, the Cas9 DNA-endonuclease enzyme and a single guide RNA (sgRNA) that is comprised of the 20 base pair (bp) target sequence, and the $77 \mathrm{bp}$ region encoding the RNA scaffolding flanked by five thymine residues coding for termination of transcription, totaling a 102 bp RNA fragment. The sequence for Cas9 is publicly available and can be found in Supplemental Material (Supplemental File C5-1). While the Cas9 amino acid sequence is largely the same regardless of the system in which it is to be used, codon optimization of the Cas9 gene for optimal translation in your particular organism improves efficiency when using a transformation that relies on translation of the supplied gene within the host. This can be accomplished if the codon bias is known for your organism; however, if codon bias information is not available, a plasmid for use of CRISPR Cas9 optimized for a closely related system can be obtained from one of the many plasmid repositories, such as Addgene.org (https://www.addgene.org/), and may work well enough in your organism. If you are using Cas9 in a eukaryotic system, it is important to include the addition of a nuclear localization signal (NLS) at the $\mathrm{C}$ or $\mathrm{N}$ terminus of the Cas9 protein to allow the Cas9 to enter the nucleus after translation (Supplemental File C5-1). Like Cas9, the gRNA scaffold code will also remain the same, unless you are working with a modified version of Cas9 designed for shorter sgRNA sequences (Xu et al., 2017).

While the Cas9 and gRNA scaffold sequences will remain relatively the same regardless of the system in which they are being used, their expression should be directed via endogenous or tested promoters for each new system. To drive the expression of the nuclear localization signal tagged Cas9, a strong promoter from your organism should be cloned upstream of the Cas9 coding region, thus yielding high-level expression. Alternatively, differentially expressed promoters can be used to express Cas9 under specific conditions. For expression of Cas9 in eukaryotes, an RNA Pol II promoter can be used; however, while Cas9 expression can utilize such promoters, a promoter for RNA Pol I or RNA Pol III must be used to drive transcription of the sgRNA component in order to avoid the addition of a 5'-cap and 3'-poly A tail. Promoters for U6 or U3 snRNAs are conventionally universal promoters for RNA Pol III that have been used with widespread success to transcribe sgRNAs in many, but not all, systems (Cong et al., 2013). For use in prokaryotes, the Cas9 encoding region can be cloned in between any conventional promoter and transcription 
terminator site. The sgRNA however needs to be cloned in exactly 10 bp downstream of the -10 Pribnow box in order to ensure that no extra base pairs are added onto the 5 ' end of the target region of the sgRNA.

An alternative approach for expression of the sgRNA component in eukaryotes is to use the organism's natural tRNA promoter to create an sgRNA/tRNA chimera (Mefferd et al., 2015). This can be done by fusing the sgRNA sequence directly downstream of a functional tRNA, followed by a termination sequence. This will allow your organism to transcribe an sgRNA/tRNA fusion product that, after posttranscriptional modification of the tRNA, will yield both a functional tRNA and the sgRNA for use by Cas9. While use of tRNA promoters can allow for expression of sgRNAs in systems with limited genomic annotation, tRNA promoters can reduce efficiency of Cas9 activity when compared to the U6 or U3 promoters (Wei et al., 2017). Even so, U6 promoters have been demonstrated to be unsuitable for driving sgRNA in some organisms. For example, U6 promoters are not used in many yeasts. Instead, at least one group has used the promoter from the Saccharomyces cerevisiae small nucleolar RNA, snR52 (Psc-SNR52), shown to be effective in a variety of yeast species (Wang et al., 2018). Furthermore, while using RNA Pol III systems is the predominant option for initiating CRISPR Cas9 technology in a new system, inventive researchers have utilized artificial enzymes to remove the transcriptional additions of RNA Pol II and prevent the sgRNA from being transported to the cytosol, demonstrating that it is possible to express a functional sgRNA using Pol II systems, if necessary (Nødvig et al., 2015; Zhang et al., 2017). Examples of this alternative Cas9 expression approach can be found in several systems developed for a variety of filamentous fungi, where efficient promoters for RNA Pol III are not well characterized (Nødvig et al., 2015; Schuster et al., 2019).These researchers used a variant of the CRISPR-Cas9 system in which the sgRNA is embedded in the middle of a larger transcript synthesized by RNA polymerase II; the sgRNA is liberated from the larger transcript in the nucleus by the action of two ribozyme sequences, 5'-end hammerhead (HH) and 3'-end hepatitis delta virus (HDV), which flank the sgRNA (Nødvig et al., 2015). The RNA Pol II promoter driving expression of this RNA is the Aspergillus nidulans gpdA promoter, a strong constitutive promoter; this is combined with the $\operatorname{trpC}$ transcriptional terminator (Nødvig et al., 2015).

Once implemented in your system, CRISPR Cas9 can be easily adapted for new targets by simply swapping out the $20 \mathrm{bp}$ target region of the sgRNA for a new target. However, even though CRISPR Cas9 is a highly robust system once implemented, it is not without its own limitations. The PAM site for Cas9 
restricts the cutting activity of the enzyme to NGG sequences, which theoretically occur 1 in every $16 \mathrm{bp}$ in the genome. Furthermore, off-target cuts are a major concern for the application of CRISPR Cas9. While the target region of the gRNA binds to 20 complementary bp, mismatch pairing is able to occur closer to the 5 , end of the RNA (Hsu et al., 2014). With these limitations in mind, the rest of this introduction will serve as a guide through the process of implementing CRISPR Cas9 in a new system, after which, I will present data on our efforts to do so in the Microbotryum genus.

\subsection{Things to consider before you begin}

Before designing a CRISPR Cas9 construct for use in a new system, there are several parameters to consider: 1) what transformation systems are available for your organism? 2) What sort of genetic changes are your trying to make in your organism? 3) What is the target for your pilot study? 4) How do you plan to ensure transcription and translation of the Cas9 enzyme? And 5) How do you plan to provide transcription of the sgRNA?

\subsection{Selecting a transformation method}

Establishing a CRSIPR Cas9 protocol for a new organism will rely heavily on the methods of transformation available for that system. This section will provide an overview of several approaches that have been used with widespread success. Each of the following methods have their own pros and cons associated with implementing CRISPR Cas9, and should be considered in the context of your organism and the goals of your research. For example, some projects may tolerate off-target cutting while others may require strict fidelity of the enzyme. Furthermore, some organisms may be limited by their genetic toolboxes to a subset of the following approaches.

\subsection{Single plasmid method}

The conventional method of implementing CRISPR Cas9 is to use a plasmid that contains all the necessary coding regions and promoters to drive transcription and translation of the Cas9 DNA-endonuclease and the associated sgRNA within the organism itself, utilizing the endogenous cell machinery to do most of the work for you. Introduction of CRISPR Cas9 in this way requires, in addition to the coding regions for the sgRNA 
and Cas9-NLS, a promoter for Cas9 expression, a promoter for the sgRNA, a selectable marker for successful transformation, and either an Origin of Replication (ORI) for prokaryotes or an Autonomous Replication Sequence (ARS) for eukaryotes to maintain the plasmid through cell division (Figure C5-1).

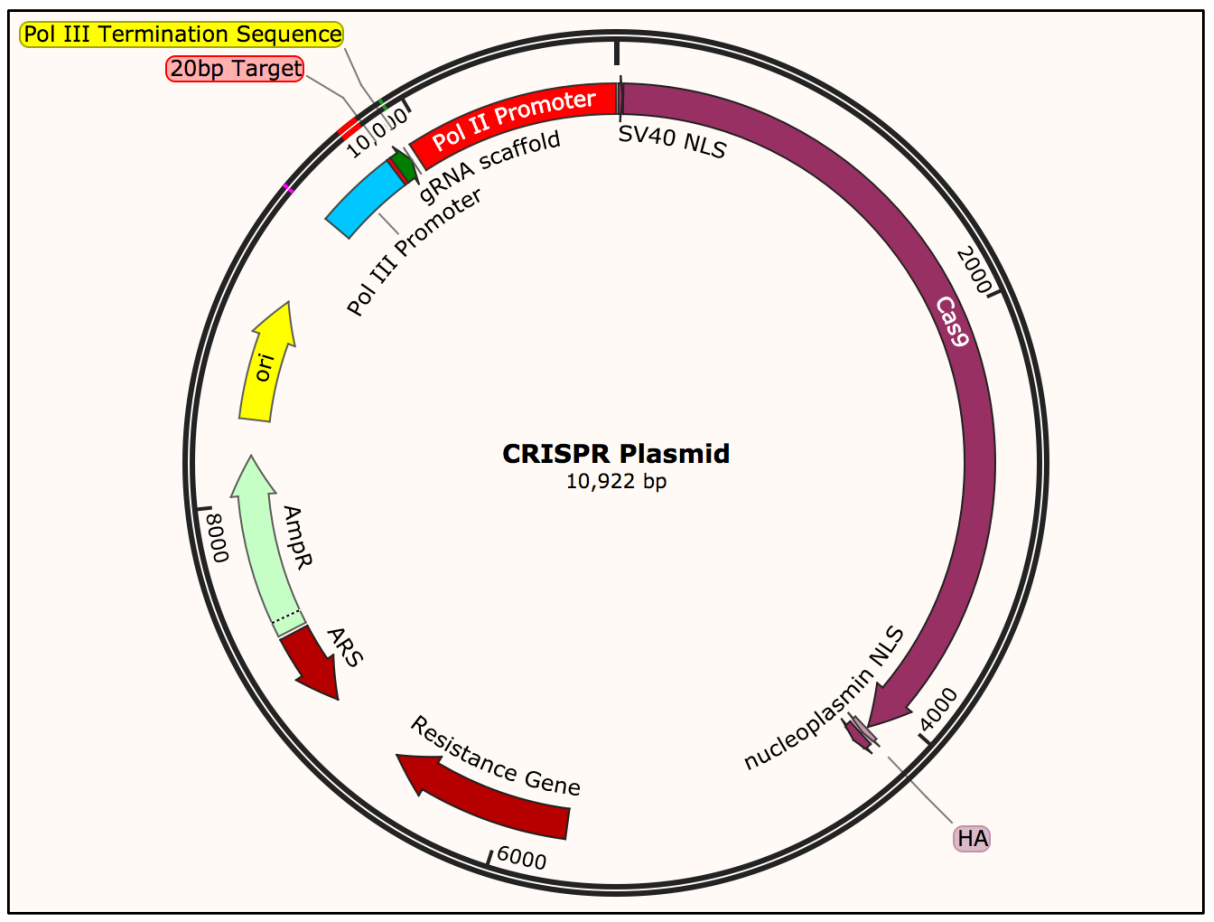

Figure C5-1 Diagram of the components required to implement CRISPR Cas9 via a single plasmid in a eukaryotic system. The components required are an RNA Polymerase III promoter, 20 base pair target, guide RNA scaffold, an RNA Polymerase II Promoter, Cas9 coding sequence with nuclear localization signal, selectable resistance gene for the transformed host, autonomously replicating system/ origin of replication. Image generated with SnapGene ${ }^{\circledR}$.

Introduction of CRISPR Cas9 via plasmid can be performed using various transformation methods, including Heat Shock Transformation or Electroporation in prokaryotes (Froger and Hall, 2007; Tu et al., 2016), Polyethylene Glycol (PEG) facilitated transformation in fungi (Liu and Friesen, 2012), biolistics in plants (Char et al., 2017; Ismagul et al., 2018), and viral infection of animal cells (Kost et al., 2005). To identify successful transformants, cells carrying the plasmid are selected using media containing the antibiotic corresponding to the selectable resistance gene on your CRISPR plasmid; such initial transformants are then 
subsequently passaged on non-selective media to allow cells to lose the plasmid during subsequent rounds of mitotic cell division. This will limit the exposure of the cells to Cas9 activity, thus lowering the risk for offtarget cuts and unwanted modifications to the genome. Cells can then be screened for CRISPR-mediated changes.

\subsection{Agrobacterium-mediated method}

While plasmid introduction of CRISPR Cas9 is the most common and straightforward method, without an ORI or ARS, selection of transformed cells would require integration of the vector into the host genome. An alternative transformation method for organisms for which an ORI or ARS is unknown is Agrobacteriummediated transformation (ATMT) (Char et al., 2017). To perform ATMT on eukaryotic cells, a Ti plasmid is used with the following components cloned between left and right T-DNA borders; an RNA Pol II promoter, the Cas9 encoding gene, a Pol III promoter, and the sequence for the sgRNA (Figure C5-2).

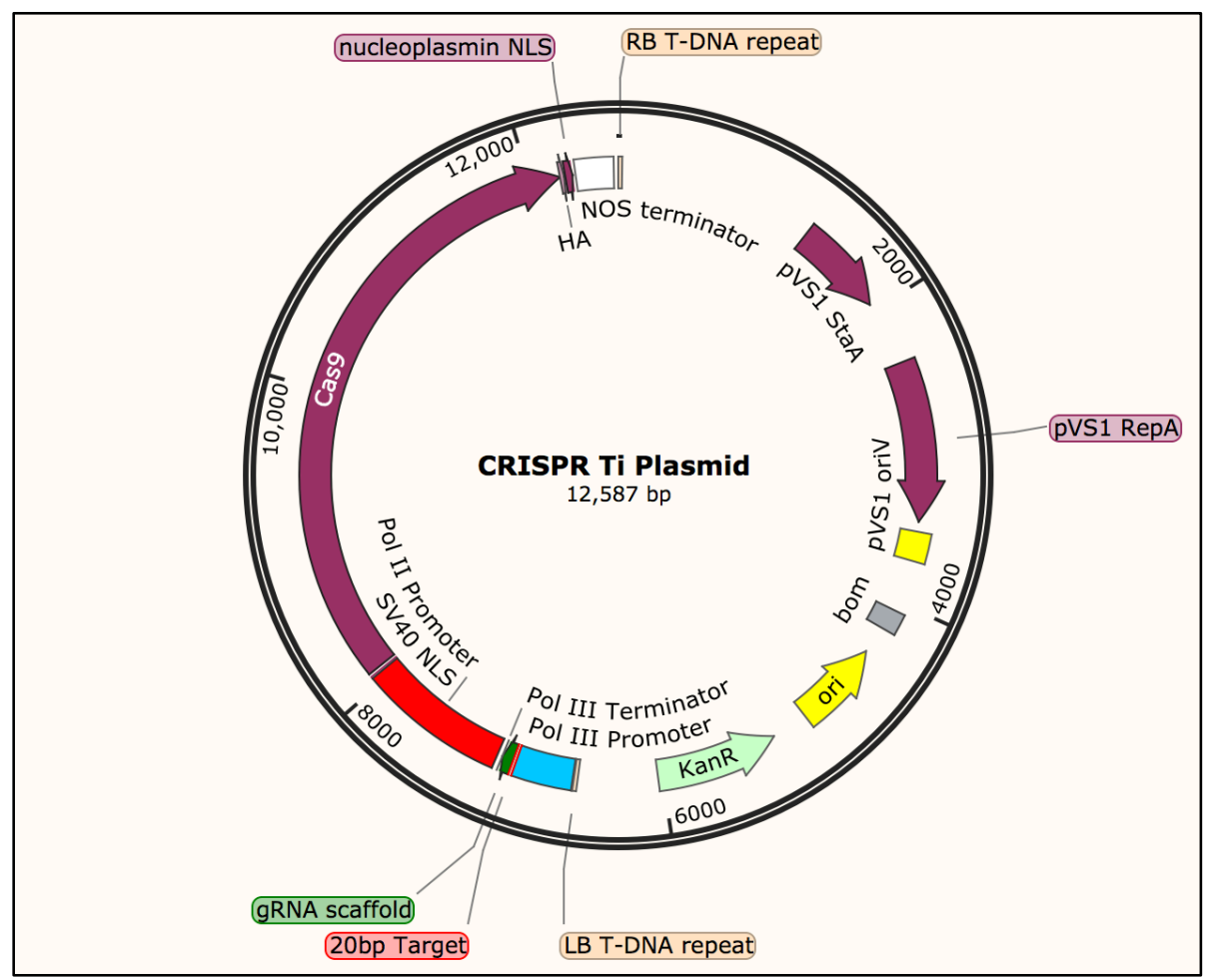

Figure C5-2 Diagram of the components required to implement CRISPR Cas9 via Agrobacterium mediated transformation in a eukaryotic system. The required components are: an RNA Polymerase III promoter, 20 base pair target, guide RNA scaffold, an RNA Polymerase II Promoter, Cas9 coding sequence 
with nuclear localization signal. These components are cloned in-between the left and right T-DNA border regions and transformed into Agrobacterium tumefaciens for transformation of various cell types. Image generated with SnapGene ${ }^{\circledR}$.

These Ti plasmids are then transformed into Agrobacterium tumefaciens, which will be used to shuttle the DNA between the T-DNA borders into the genome of your host. To do so, transformed Agrobacterium are plated together with the host cells on mating agar containing acetosyringone. The acetosyringone causes the Agrobacterium to form conjugative structures that will transfer the T-DNA cassette into essentially any cell type (Char et al., 2017; Kunik et al., 2001; Toh et al., 2016; Liu et al., 2017). While this method eliminates the need for an ORI or ARS, the CRISPR Cas9 components become permanently incorporated into the host genome, and thus removal of Cas9 via selection-free cell passaging is no longer possible. This ultimately increases the risk for off-target effects of Cas9; however, measures can be taken to reduce the risk of offtarget hits when using ATMT. One solution is to add a second sgRNA, in addition to the sgRNA for your target of interest, one that targets the Cas9 gene itself, effectively acting as a kill switch and limiting the exposure that the functional Cas9 enzyme has with the host genome. While this method reduces off-target hits, it also reduces the efficiency of on-target cuts. Regulation of Cas9 with a differentially expressed promoter is another option for regulating Cas9 by controlling when the enzyme is being expressed through the use of particular media types. It is also important to consider that the random integration of the CRISPR cassette into transcriptionally active regions of the host genome may itself lead to gene interruptions and should be screened before attributing phenotypic changes to Cas9 action.

\section{5 mRNA-encoded Cas9 method}

Another alternative for systems without a viable means of plasmid replication is to transform cells with mRNA coding for the Cas9 enzyme. Like the plasmid method, the mRNA must also include the sequence for an NLS tag in order for the translated protein to be shuttled back into the nucleus; however, unlike the plasmid method, the mRNA itself does not require transportation into the nucleus and, of course, the mRNA is far less stable in the cell. While at first glance, a lack of stable expression of Cas9 may seem inefficient due to the limited window for expression and action of the Cas9 enzyme, the limited exposure of cellular 
DNA to the Cas9 product reduces the risk of off-target cuts, therefore, making this method advantageous for stringent projects that cannot tolerate off-target cutting. This method is the predominant approach for animal systems and is often performed via in vitro production of mRNA followed by microinjections of a mixture of sgRNA and the Cas9 mRNA product into the cells (Figure C5-3).

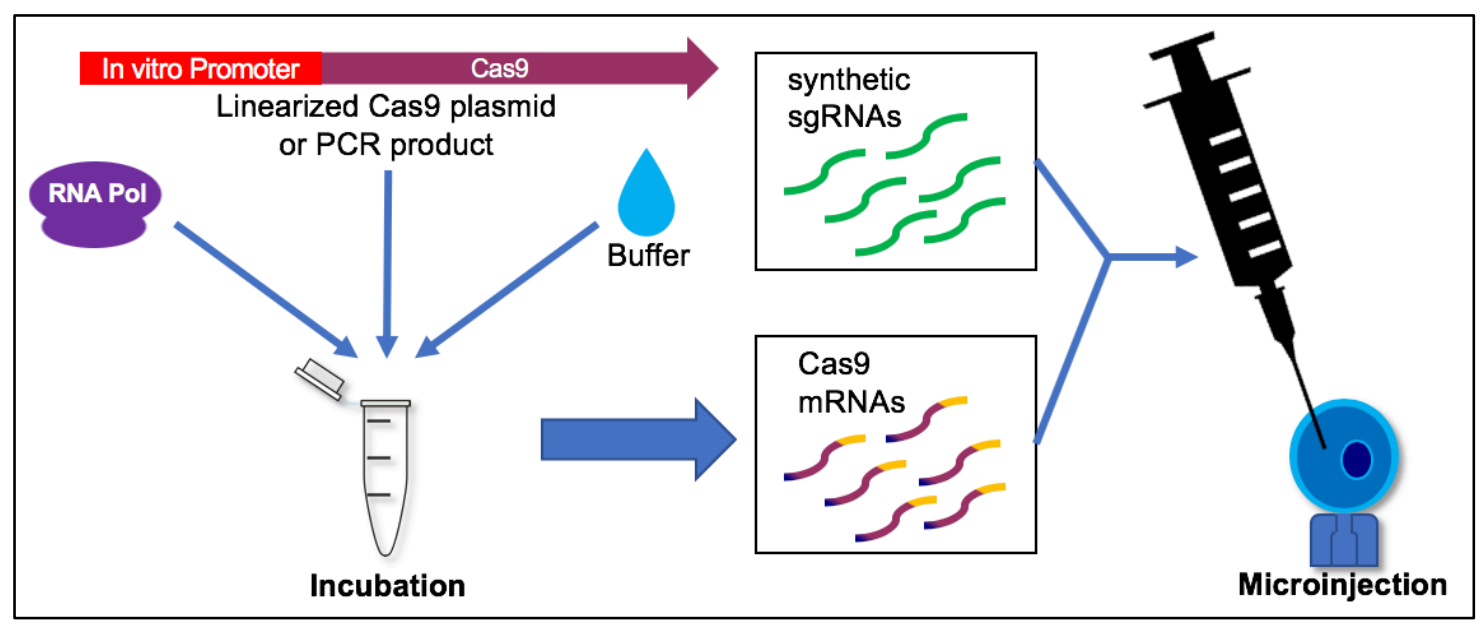

Figure C5-3 Introduction of CRISPR via mRNA-encoded Cas9. Cas9 plasmid with a promoter modified for use with in vitro mRNA synthesis kits can be linearized or used as template for PCR to generate the template for mRNA synthesis via RNA Polymerase. This process differs by kit, but usually includes an incubation period with a master mix or buffer, followed by an RNA purification step before use for microinjections into cells.

Transformation of cells using the mRNA-encoded Cas9 CRISPR method has the advantage of limiting the exposure of the cells to Cas9, since both mRNA and Cas9 protein lead to fewer opportunities for off-target cuts; however, genetic modification efficiency is also reduced when compared to more stable methods of Cas9 expression. In addition to reduced efficiency, this method also requires the tedious practice of isolating and handling RNA, which requires the use of kits including RNase-free buffers and lab equipment, as well as sterile RNA workspaces. 


\subsection{Purified Cas9 method}

For systems where the genetic information to drive expression of engineered genes is unknown, the following approach can be used. Rather than driving expression of the sgRNA or Cas9 in the host via a plasmid or mRNA construct, one can now purchase commercially available recombinant Cas9 proteins from various sources. Alternatively, a protein expression plasmid utilizing a T7 promoter (pET plasmid) can be used to express and purify the Cas9 from bacteria, using a Histidine tag (Cas9-NLS-6xHis). E. coli DE3 cells can be easily transformed with these pET plasmids and grown with IPTG to induce production of the Cas9 enzyme. Cells can then be sonicated to obtain a Cas9-rich lysate for Ni column purification. The purified stock of enzyme can then be combined with synthetically produced sgRNAs and transformed into the host cells (Foster et al., 2018) (Figure C5-4).

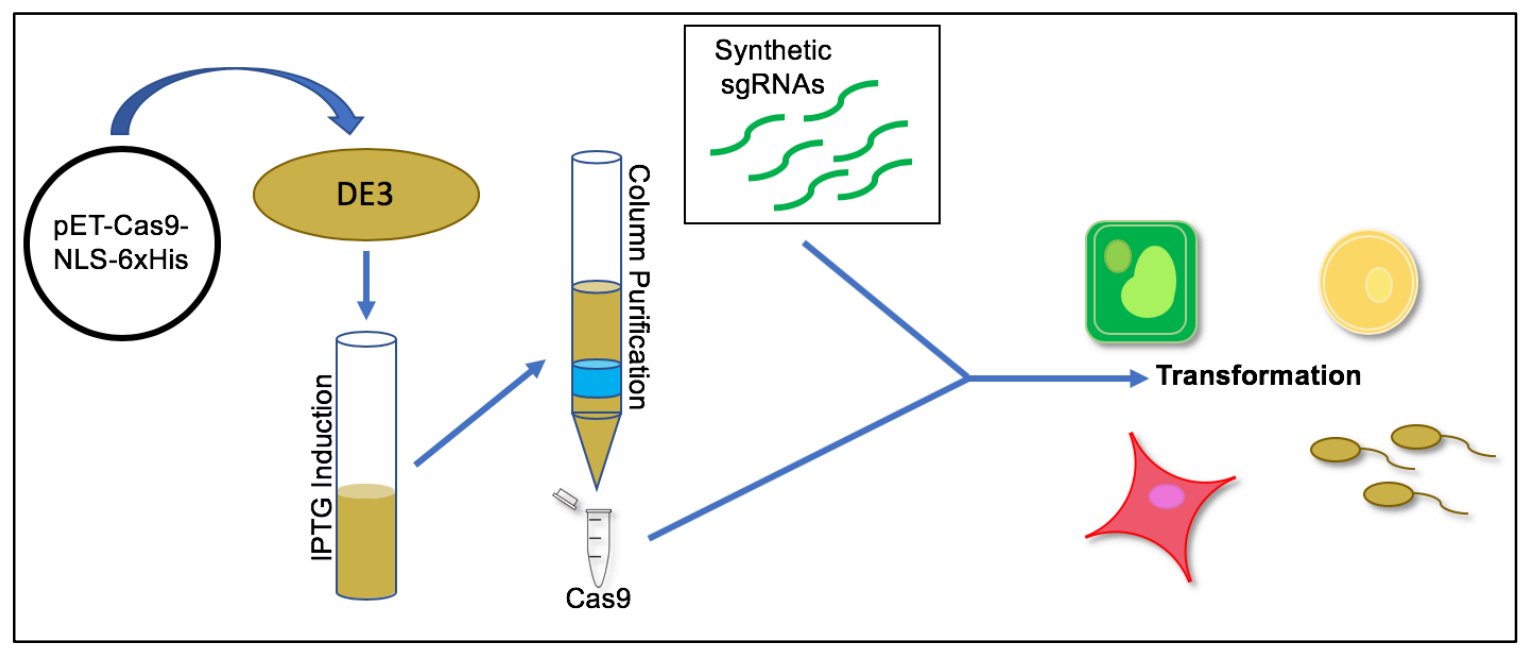

Figure C5-4 Protein purification of Cas9 for introduction to various cell types along with synthetic

sgRNAs. IPTG-inducible Cas9 plasmids can be transformed into DE3 Escherichia coli cells and induced overnight in $2 \mathrm{X}$ growth media. A $6 \mathrm{X}$-His tag fused to the end of the Cas9 DNA-endonuclease enzyme allows for its purification on nickel (Ni) columns. Synthetically produced sgRNAs can then be mixed with the Cas9 enzyme prior to introduction to various cells types.

Alternatively, as indicated earlier, for labs with the necessary funds, purified lyophilized Cas9 enzyme can be purchased from companies such as New England Biolabs, IDT, or System Biosciences ${ }^{\mathcal{O}}$ pre-made and ready for use. While this method requires the least amount of genetic information for the organism with 
which you are working, and has one of the lowest chances for off-target effects, this method has comparatively low transformation efficiency compared to the other methods and the purchase of sgRNA oligos can be expensive. Newer approaches for the synthesis of RNAs in vitro have reduced the cost, making this a more viable approach for a larger breadth of labs, by taking advantage of the hydrogen bonding involved in the formation of loop structures to attach the sgRNA to the Cas9 enzyme to produce the sgRNA as two separate 60 base pair RNA oligos and relying on complementary sequences to hold them together before duplexing with the Cas9 enzyme (Figure C5-5). These two separate RNA strands are the crRNA, which includes the desired host target sequence, and tracrRNA, which contains the RNA scaffolding required to bind to the Cas9 endonuclease. By reducing the overall length of synthesis needed for each RNA oligo, price can be dramatically reduced. Furthermore, tracrRNAs are specific to the particular endonuclease to which they bind and therefore do not need to be resynthesized every time a researcher wishes to change targets. Instead, a new crRNA can be synthesized and complexed with the previous stock of tracrRNA, further reducing costs.

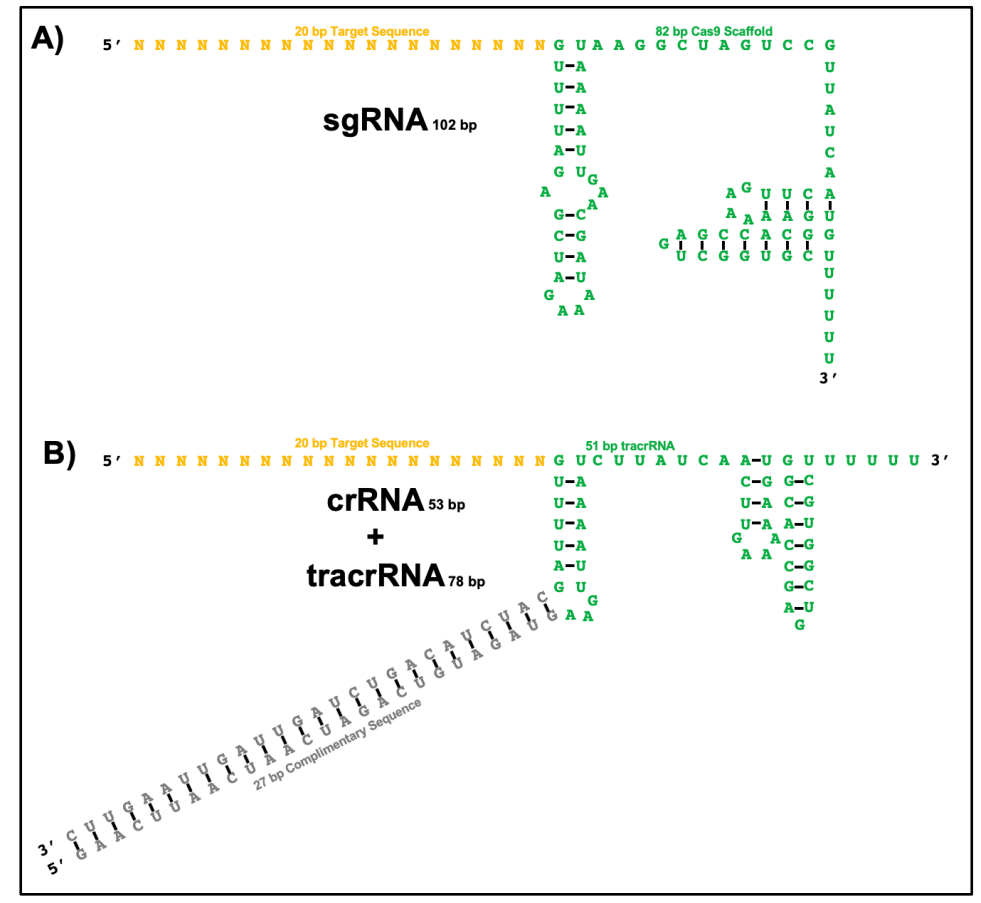

Figure C5-5 Diagram of the differences between sgRNA and crRNA/tracrRNA molecules. Hydrogen bonding arrangement of the traditional sgRNA used for CRISPR Cas9, A, and the more recent two oligo duplex using crRNA and tracrRNA, B. 
If cost still remains an issue for the researchers, another option is to produce these sgRNAs on your own using an RNA synthesis kits, similar to the ones mentioned in the mRNA-Encoded Cas9 method.

\subsection{Selecting a suitable Cas9 variant}

The Cas9 DNA-endonuclease enzyme, originally isolated from Streptococcus pyogenes (SpCas9), has since been modified in several ways to alter its function and fidelity within the cell. Cas9 enzymes have two functional nuclease domains, the single nuclease domain $\mathrm{HNH}$, which acts to cut the strand complementary to the gRNA, and RuvC, comprised of 3 subdomains, responsible for cutting the non-complement strand (i.e., the strand with the PAM site). Together, these active domains work together to create a blunt cut across the double stranded DNA target, 3-4 bp upstream of the PAM site. By changing the amino acid composition of these functional domains, Cas9 can be used for other types of genetic modification in your system (Figure C5-6).

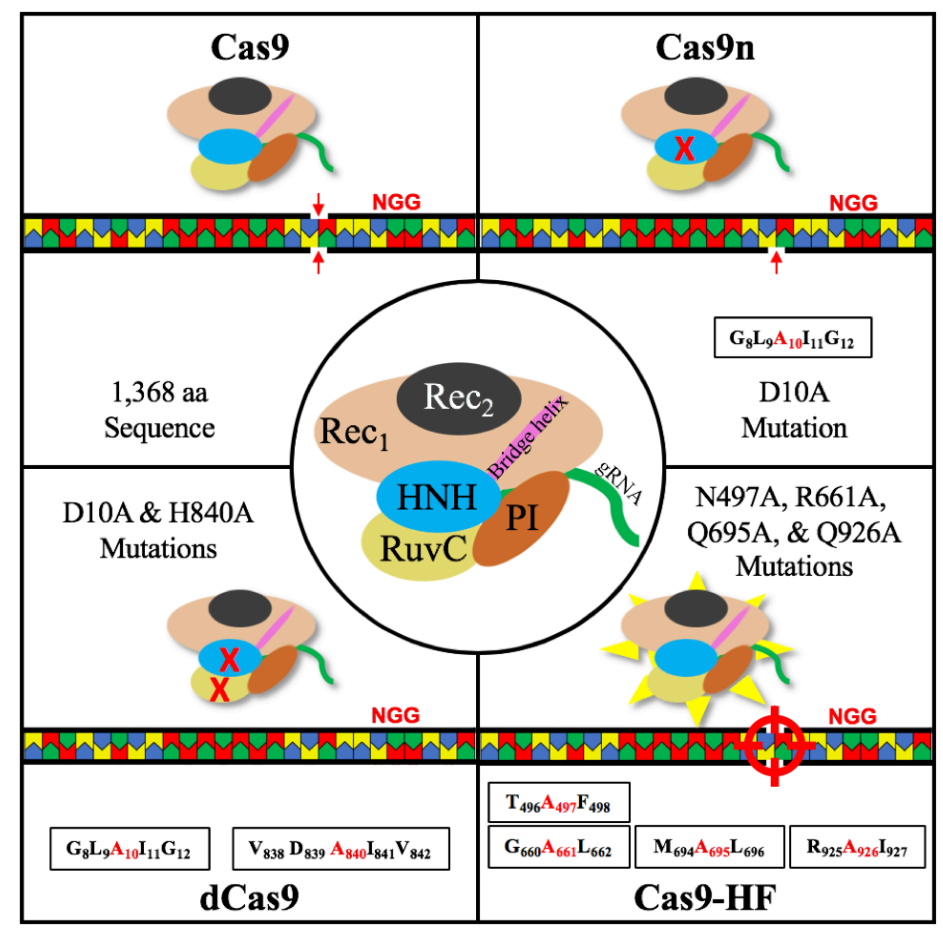

Figure C5-6 Illustration of various Cas9 mutants and their amino acid substitutions. The Cas9 DNAendonuclease enzyme, color coded by protein domains, center. The traditional double stranded cut-inducing SpCas9 enzyme, top left. The HNH-nickase Cas9 mutant (Cas9n), top right. The HNH-RuvC double 
knockout mutant for enzymatically dead Cas9 (dCas9), bottom left. And, the high-fidelity Cas9 variant (Cas9-HF), bottom right.

\subsection{Cas9 endonuclease}

Cas9 functions to generate double stranded cuts 3-4 bp upstream of the protospacer adjacent motif (PAM) site of your target (Cong et al., 2013). These double stranded breaks are then repaired through either nonhomologous end joining or homology directed repair within the cell (Hsu et al., 2014), processes that are error prone and often lead to the removal or addition of nucleotides at the ends of the breaks. The addition or deletion of nucleotides before the strands are reconnected induces a frameshift that can lead to a change in reading frame and the introduction of premature stop codons, ultimately changing the peptide sequence and truncating the translated protein, giving Cas9 its characteristic knock-out function. In the event that the cell does manage to repair the double stranded break appropriately, the target site for the Cas9 is also repaired and further cuts can be made until there is a change to the sequence.

In addition to generating frameshifts, double stranded breaks induced by Cas9 have also been demonstrated to dramatically increase the rate of homologous recombination in cells (Miki et al., 2018). Selectable markers with upstream and downstream complementary regions to either side of the targeted double stranded cut in the organism's genome can be introduced along with Cas9 to facilitate homologous directed repair of the double-stranded break to insert a selectable marker into the coding region of the gene you wish to knockout (Miki et al., 2018) (Figure C5-7). 


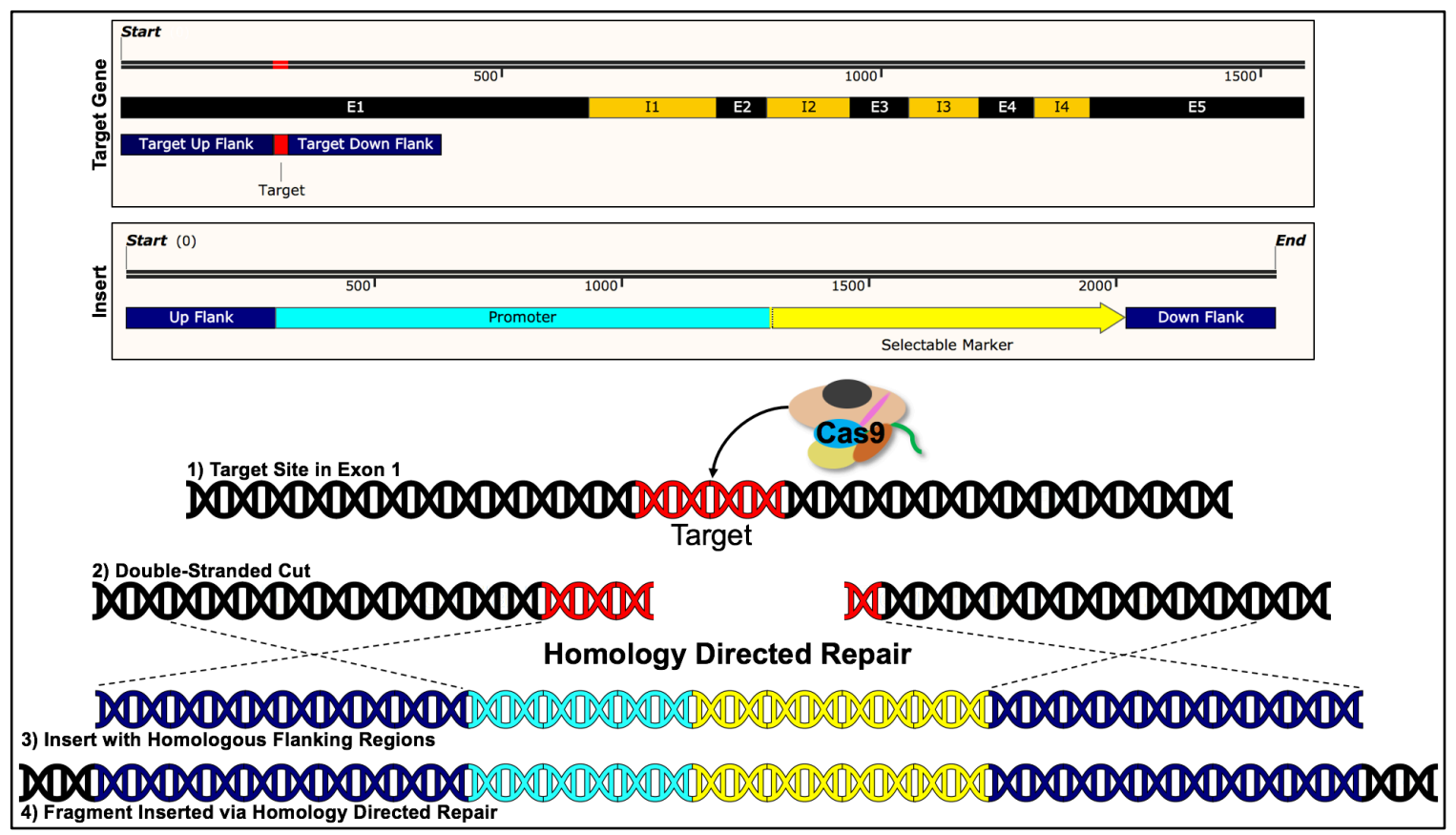

Figure C5-7 Homology directed repair of a Cas9-induced doubled-stranded break in the first exon of

a target gene. 1) the targeting and binding of Cas9 to the first exon region of the target gene. 2) The endonuclease activity of Cas9 generates a double-stranded break 3-4 bp upstream of the PAM site of the target region. 3) The homologous flanks of the insert are used as a template for homology directed repair of the double-stranded break. 4) The repair results in the addition of the DNA between the up and down flank homologous regions, disrupting the target gene and allowing for expression of a selectable marker. The top two images were generated with SnapGene ${ }^{\circledR}$.

\subsection{Cas9n nickase}

By changing the amino acid sequence of one of the nuclease domains of Cas9, function can be altered to that of a nickase enzyme, where one nuclease domain is still functional and cuts one side of the DNA, but the other domain does not (Chew et al., 2016). Nickase Cas9 mutants (Cas9n) can be used with a pair of sgRNAs to generate cuts on either side of a target DNA sequence several base pairs apart, effectively allowing for the strands to separate with regions of overhangs that can then be utilized for site specific insertions of DNA. Cas9n can thus be used for incorporation of transgenes into site-specific loci, or used to insert screenable markers, such as GFP, to disrupt function and screen for knockouts within a system. While Cas9n utilizes two sgRNAs, thus theoretically doubling the risk of off-target cuts, the lack of complete double stranded 
breaks reduces the risk of off-target changes in the genome, as nicks are easily repaired without mutation. This makes Cas9n a great approach for groups seeking to minimize off-target effects in their system. Because this system utilizes two sgRNAs and relies on strand separation of regions held together by hydrogen bonds between the two nicks, the PAM sites of both targets should be on opposite strands and no more than 30-40 bp apart in order to effectively allow the two nicked strands of complementary DNA to separate. Increasing the distance between nicked strands increases the number and consequently the strength of the hydrogen bonds, ultimately decreasing the likelihood that the strands will separate.

\section{$1.10 \mathrm{dCas} 9$ for CRISPRi}

Another type of Cas9 mutant can be made by disrupting both nuclease domains, generating an enzymatically dead variant of Cas9 (dCas9) (O'Geen et al., 2017). While this Cas9 mutant is unable to cut the target, it is still able to bind specifically to the target site. This variant of Cas9 can be used to either interfere with cellular processes at the transcriptional level (CRISPRi), or in conjunction with activation domain attachments to act as an inducible activation delivery system. dCas9 has also been used as a vehicle for fluorescent tags for realtime DNA imaging in cells (Chen et al., 2013).

\subsection{High fidelity Cas9 variants (Cas9-HF, eSPCas9, and xCas9)}

In addition to enzymatic ability, another thing to consider when selecting a Cas9 variant is the importance of preventing off-target cuts in your system. High-Fidelity variants of Cas9 have been generated for use in systems that may need increased specificity, such as eSpCas9 and various forms of Cas9-HF (Kim et al., 2017; Kleinstiver et al., 2016). These Cas9 variants have amino acid substitutions that reduce the likelihood that annealing of the gRNA with mismatches at the 5' end of the target sequence will induce enzymatic activity in the Cas9 enzyme. In addition to the creation of high-fidelity Cas9 variants, recent researchers at Harvard and MIT have generated SpCas9 mutants through directed evolution that can utilize multiple different PAM site sequences (xCas9), further increasing their use for very site-specific purposes (Hu et al., 2018). Not only can these xCas9 variants recognize PAM sites other than NGG (e.g., NG, GAA, and GAT), but they also surprisingly demonstrated increased editing specificity (Hu et al., 2018). These High-Fidelity Cas9 variants can additionally be modified to operate with any of the aforementioned Cas9 enzymatic 
abilities as well, although some of them have modified components of the sgRNAs that need to be taken into consideration when designing a target, such as alterations to the recognized PAM sequence in $\mathrm{xCas} 9$ (Hu et al., 2018). The sequences with appropriate substitutions for each of the aforementioned Cas9 variants can be viewed in the Supplemental Materials (Supplemental File C5-1).

\subsection{Transposon Associated CRISPR Cas9}

One group has created yet another form of CRISPR Cas9-mediated transformation that is able to insert DNA into a targeted area without the need for any cutting of the host genome at all (Klompe et al., 2019). This CRISPR approach utilized the specificity of the Cas9 sgRNA chaperone and a transposable element system to insert DNA without the risk of unintentional degradation to the host genome, making CRISPR applicable to systems with poor repair machinery, such as certain bacteria, where double-stranded breaks are fatal to the cell.

This idea of fusing CRISPR Cas9 with other gene editing tools has also been used to generate enzymes capable of site-specific nucleotide substitutions. By tethering cytidine or adenine deaminase to Cas enzymes, CRISPR can be used to introduce C-to-T or A-to-G changes to specific regions of a gene without the need for cutting the genome (Gaudelli et al., 2017; Zheng et al., 2018). The ability to make site-specific single nucleotide changes to DNA allows the user to change particular amino acids in proteins, a tremendous tool for those performing directed experimental evolution studies or investigating the function of proteins. While CRISPR-mediated base editing is currently limited to C-to-T changes and A-to-G changes, new CRISPR applications like the aforementioned Cas9-transposable element are expanding the capacity for genome editing without the need for cutting (Komor et al., 2016).

\subsection{Selecting a target}

One of the more challenging aspects to using CRISPR Cas9 for the first time is screening for cells with the intended genetic modifications. Cas9 DNA-endonuclease activity leads to double-stranded breaks in the

primary DNA of an organism; however, not every cut leads to elimination of function. When repairs are made to the double-stranded breaks in the cell, some of the DNA will be successfully put back together with the addition or deletion of base pairs in triplicate, e.g., three nucleotides are deleted in a unimportant region 
of the coding DNA. When this occurs, function of the targeted protein may persist. Furthermore, targeting of an intronic region in eukaryotes will also likely not lead to changes in protein function. Therefore, selection of an appropriate region of the DNA for the gene you want to disrupt is imperative. This can be accomplished by either targeting the active site for a protein, where even single amino acid insertions or deletions in the protein can cause disruption, or selecting a target as far upstream in the coding region as possible to increase the impact of induced frame shifts.

To determine whether CRISPR Cas9 has successfully altered the target in your system, a method for screening transformed cells will be needed. While genetic sequencing of the target region is a necessity to demonstrate successful genetic modification, screening thousands of colonies to find a successful knockout can be costly and time consuming. Therefore, pilot studies for implementing CRISPR Cas9 in a new system would do well to start by targeting a gene that leads to an easily discernible phenotypic alteration. Inducing a color change or changing an organism's ability to grow on certain media types is a great way to initially implement CRISPR Cas9 in your organism, which can then be altered later on for different targets. Targets that provide easily-identifiable phenotypic changes can also be used in tandem with future targets that may not lead to an observable change in order to select for cells in which the active CRISPR Cas9 has been successfully delivered, reducing the number of cells where DNA sequencing will be required to identify changes. If you are implementing CRISPR in a system that does not have the genetic information for an observable phenotypic change, the organism can first be transformed with a marker, such as GFP, that can then be subsequently targeted with Cas9 to test CRISPR in your system (Watters et al., 2018). Alternatively, Cas9n mutants can be used to insert markers like GFP into the coding region of the gene you wish to knockout.

\subsection{Selecting a promoter for Cas 9}

Depending on the transformation method available to your system, different strategies can be used to drive Cas9 expression. To drive the transcription of plasmid-encoded Cas9, a constitutive promoter from a known gene from your organism can be cloned upstream of the coding region for a codon-optimized Cas9 variant. After selection of successful transformants, the Cas9 plasmid can be eliminated by repeated passaging using non-selective media (Schuster et al., 2016). When using a constitutive promoter, Cas9-HF is recommended 
since the Cas9 will be constantly expressed during the passaging process and may lead to off-target cuts. An alternative to using a constitutive promoter for the Cas9 plasmid is to use a differentially expressed promoter that can upregulate expression of Cas9 on certain media types during transformation/selection and then be downregulated during the passaging step of the process. RNA-seq data can be used to identify promoters that drive the transcription of certain genes on rich media, but have significantly reduced transcription on minimal media, or vice versa.

Organisms for which the use of plasmids are not suitable, and more permanent means of transformation like homologous recombination or Agrobacterium-mediated transformation are used, differentially expressed promoters are recommended. Off-switches can also be used to conditionally inhibit Cas9 activity after its initial introduction into a system, giving the Cas9 time to make changes before it is turned off (Pawluk et al., 2016).

In systems where the Cas9 enzyme is purified from bacteria and added separately, commercially produced pET Cas9 plasmids are available that use an IPTG-inducible promoter for expression of Cas9 in large abundance within E. coli cells. For extraction of Cas9 in this manner, pET Cas9 plasmids with 6xHis tags can be obtained (e.g., Addgene.org) to purify Cas9 enzyme from bacterial lysate using a nickel flow column.

\subsection{Selecting a promoter for the sgRNA}

Like the Cas9 encoding region, a promoter is needed to drive transcription of the sgRNA that includes your targets and the scaffold for adherence to the Cas9 DNA-endonuclease. Transcription of sgRNAs in prokaryotic cells often uses a promoter modified with an SpeI restriction site to allow for easy exchange of the downstream target site later on. Transcription of the sgRNAs in eukaryotic systems needs to avoid adding a 5' cap or 3' poly-A tail to the RNA product, a feature of RNAs produced by RNA Polymerase II. The U6 promoter offers a somewhat universal opportunity for many, but not all, eukaryotic systems, although it requires the 5' end of the sgRNA target to begin with a G. Another potential drawback of the U6 promoter is that, in some cases, the U6 RNA itself contains regulatory sequences that can affect expression (Reich et al., 1990). For systems where the U6 promoter is not able to be utilized, use of a tRNA/sgRNA chimera is a suitable alternative to drive expression of the sgRNA by fusing it to the end of a highly expressed tRNA in 
your system (Mefferd et al., 2015). Post transcriptional modifications to the tRNA will then separate the two, leaving the cell with a functional copy of both the tRNA and your sgRNA (Mefferd et al., 2015) (Figure C58). While uncommon, it is possible to drive expression of sgRNAs as an mRNA using polymerase II (Zhang et al., 2017) or as polycistronic miRNA (Xie et al., 2017).

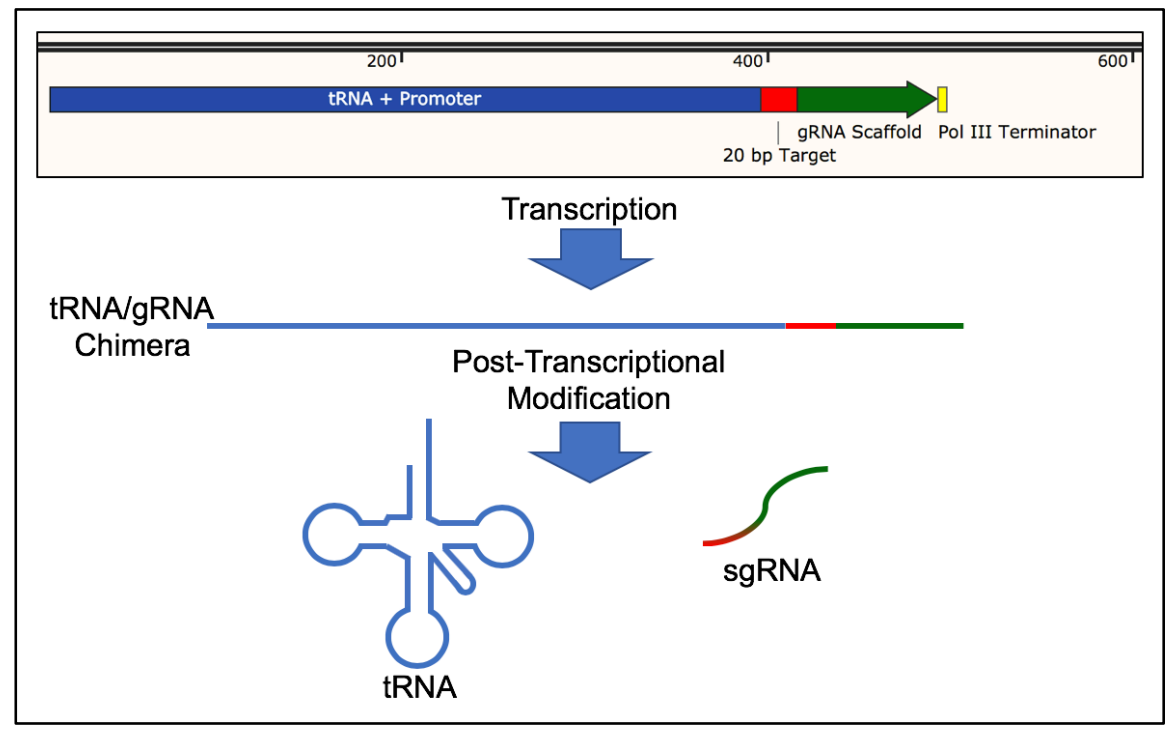

Figure C5-8 Endogenously driven synthesis of a sgRNA via fusion with a native tRNA. The $\operatorname{sgRNA}$ sequence flanked by an RNA Polymerase III terminator is cloned in place of the tRNA termination signal. Once RNA Polymerase III begins transcription of the tRNA coding sequence, it will continue through the 20 base pair target sequence and guide RNA scaffold, synthesizing a tRNA/gRNA chimera. Post-transcriptional modifications, including folding of the tRNA and processing via RNase Z, will generate a functional tRNA and result in the release of the sgRNA oligo.

\subsection{Transformation and confirmation of alterations to target genes}

Identification of successful mutations to the target region depends on the method of delivery of Cas 9 into an organism, as well as which targets were used. For investigators who elect to utilize a plasmid containing the coding region for both the sgRNA and Cas9, the selectable marker on the plasmid is the first step in identifying transformants. Using a plasmid that has a resistance gene (e.g., conferring resistance to ampicillin or kanamycin in bacterial systems, hygromycin B or carboxin for fungal systems, glufosinate, glyphosate, and kanamycin for plants, blasticidin S and thymidine kinase in mammalian cells), will allow for selection 
on media containing the drug for cells that have received the CRISPR Cas9 plasmid. Selectable markers are also possible for genomic transformations with ATMT and baculoviral transformation (Char et al., 2017; Hindriksen et al., 2017).

Because not all successful cuts with Cas9 disrupt the targeted protein, additional screening is required to identify cells with successful knockouts. For pilot studies, knocking out a gene that causes an observable phenotypic change without killing the organism is recommended. These sorts of changes can then be used in tandem with other sgRNA targets for future studies to select for cells with known functional Cas9 activity. However, in-frame deletions (i.e., in deletions in multiples of $3 \mathrm{bp}$ ), unless in coding regions for catalytically important amino acids, may not lead to disruption of the functional product. These in-frame deletions should theoretically be expected to occur randomly in $33.3 \%$ of cuts. Therefore, regardless of selection and screening, all Cas9-mediated changes should be verified via sequencing of the targeted region. Because double stranded cuts can lead to large segment deletions, to perform PCR at the target site, it is recommended to use primers that are least 100 base pairs upstream and downstream of the cut. When using Cas9n for inserting genes into a target region of the genome, screenable markers like GFP can be inserted to screen transformants for successful incorporation of the gene fragment. This can be followed up with sequencing to verify insertion of the marker into the proper location. In instances where a double mutant CRISPRi system is employed, qRT-PCR can be used to identify whether repression of the target gene has been achieved.

\subsection{Changing targets and targeting multiple genes}

One of the more exciting aspects of CRISPR Cas9 technology is the ability to target multiple genes using the same Cas9 enzyme. Because the sgRNA with an RNA Pol III termination sequence is only $102 \mathrm{bp}$ in length, plus a Pol III promoter, several sgRNA cassettes can be included on a single CRISPR plasmid (Sekine et al., 2018), or simply added in groups together with mRNA encoded Cas9 or purified Cas9 enzyme without need to modify the enzyme. Another convenience of the system is the ease of changing sgRNA for new targets. Once CRISPR Cas 9 has been established in your system, only the 20 bp target sequence needs to be changed in the sgRNA coding region to use the plasmid for a new gene. This can be easily and affordably accomplished with a $60 \mathrm{bp}$ oligonucleotide consisting of the $20 \mathrm{bp}$ target in the middle flanked by $20 \mathrm{bp}$ of 
complementary sequence to the Pol III promoter upstream and $20 \mathrm{bp}$ of complementary sequence to the gRNA scaffold downstream; these pieces are then put together using Gibson overlap PCR, or variations thereof, and cloned into the CRISPR plasmid (Figure C5-9) (Gibson et al., 2009).

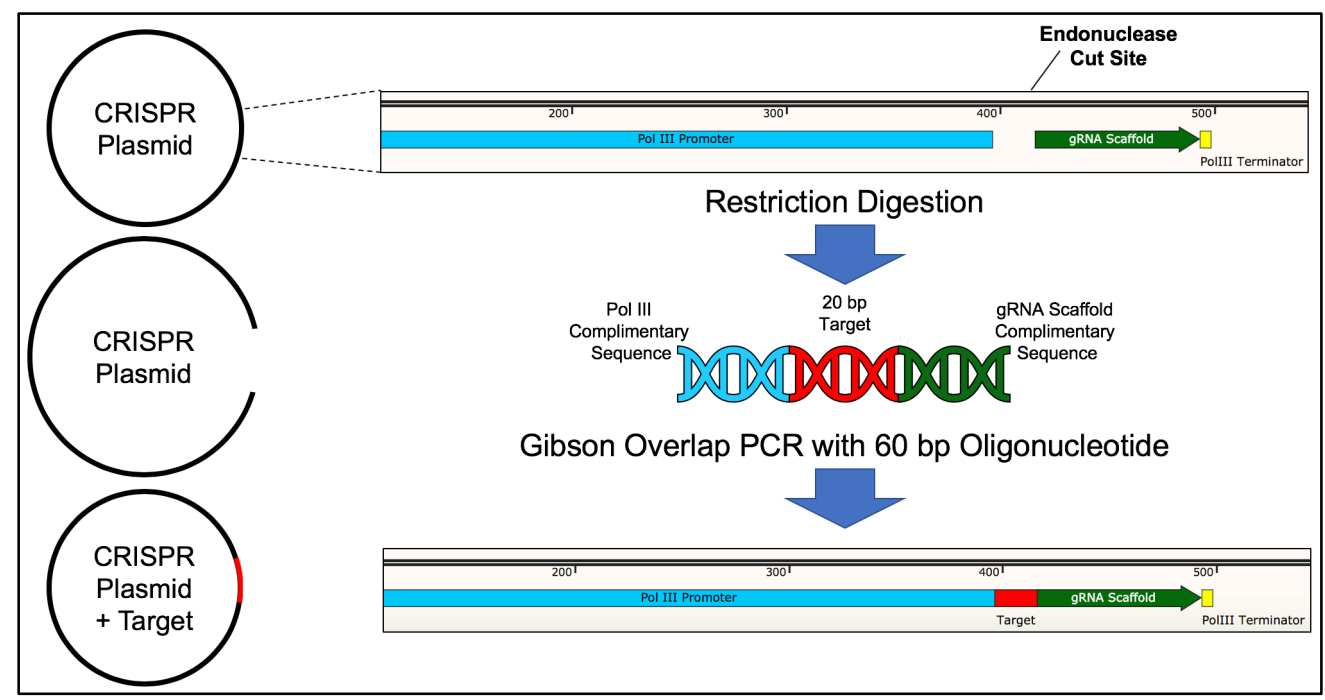

Figure C5-9 Illustration of easy target replacement in CRISPR Cas9 plasmid constructs. Insertion of a new target sequence via restriction digestion between the RNA Polymerase III promoter and guide RNA scaffold, followed by Gibson overlap PCR with a synthetically generated $60 \mathrm{bp}$ oligonucleotide. The $60 \mathrm{bp}$ oligo is comprised of three 20 bp components: 20 bp of complementary overlap sequence to the promoter, $20 \mathrm{bp}$ of the desired target, and $20 \mathrm{bp}$ of complementary overlap sequence to the gRNA sequence. Gibson overlap PCR of the digested CRISPR vector with the 60 bp oligo allows for easy cloning of new targets inframe with the gRNA scaffold for use with Cas9.

For multiple targets, one can simply create several cassettes with different targets and amplify the product with primers that include appropriate cut sites for insertion into the plasmid. While modification of a CRISPR plasmid to target multiple genes can be done with ease, the likelihood of generating knockouts for all of the targets decreases with the number of targets being used. However, when considering the amount of work that would be necessary to sequentially introduce multiple genomic changes into a single strain of your organism, the additional screening to find cells with mutations in all targets is a much better alternative to other gene editing procedures. To estimate the likelihood that all targets would lead to knockouts in your system where 
CRISPR Cas9 has been established, simply take the theoretical likelihood that a single cut will lead to a frameshift in the target gene $(66.7 \%)$ and raise it to the power of how many targets you are using (e.g., for 3 targets the theoretical likelihood of finding a cell with all three genes knocked out is approximately $30 \%$, while targeting 5 genes that would theoretically result in a quintuple knockout in roughly $13 \%$ of cells). Odds of success can be improved if the sgRNA target is in a coding region for the active site of a protein, where even in-frame deletions will lead to misfunction of the protein. Interestingly, because one would expect to have situations where some of the targeted genes are knocked out while other targets remain functional, the same single plasmid could additionally be used to test an array of combinations of functional genes followed by sequencing to determine which genes function under certain conditions, generating an extensive wealth of knowledge with a single transformation.

\subsection{Checking for off-target cuts}

While CRISPR Cas9 cuts with high specificity, especially the eSpCas9 and Cas9-HF variants, off-target cuts are possible and should be ruled out before associating phenotypic outcomes to a particular knockout in the cell. To understand the potential for off-target cuts, it helps to understand how Cas9 enzymatically operates. While the target sequence is used to bind to complementary sequence and chaperone Cas9 to the target region, one might expect the target to bind randomly in the genome once every $4{ }^{20} \mathrm{bp}$ (i.e., $1 / 1 \mathrm{E} 12 \mathrm{bp}$ ), a level of certainty that for most organisms means a single site in the genome. This expectation is only true under the assumption that the genome is completely random and that there is no mis-annealing of the target sequence to the complementary sequence in the genome; however, research has demonstrated that the target sequence is much more specific at the 3' end, next to the PAM site, than at the 5' end (Hsu et al., 2014). While the base pairs at the 3' end of the target region are incredibly specific, the base pairs at the 5' end are not required for the conformational change of Cas9. Therefore, off-target binding is far more likely to occur in sequences with mismatches at the 5' end of the target. Additionally, Cas9 occasionally binds to sequences upstream of

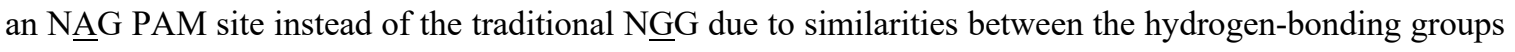
of purines (Hsu et al., 2014). To account for these potential mismatches, online tools such as E-CRISP Design created by the German Cancer Research Center are available to screen the genomes of organisms for potential 
mismatches to help you create the optimal gRNA target for your sgRNA complex (http://www.e-crisp.org/ECRISP/).

While these tools are helpful for reducing your risk of off-target cuts, whole genome sequencing for off-target cuts is the only way to be certain no off-target cuts have been made. If, like most researchers, the resources for screening multiple genomes for off-target cuts are not available to you, other methods can be used to either identify localization of the Cas9 within your cells, or to simply increase the certainty that observed phenotypic changes are due to the targeting of a particular gene, and not due to off-target cuts. To identify potential off-target cuts, a mismatch-detection nuclease assay can be performed (Wu et al., 2014). Cas9n could also be used to identify off-target regions via insertion of a bar code-like sequence that is not naturally found in your organism, followed by PCR to amplify regions where the short sequence is inserted. Multiple targets of the same gene can also be used sequentially to increase certainty that observed phenotypic changes are due to the targeted knockout. If the same phenotype is observed for all knockouts of the different areas of the target gene, using different target sequences, then the likelihood that the phenotypic changes are due to off-target interactions is greatly reduced.

\subsection{CRISPR checklist}

When it comes to implementing CRISRP Cas9 in difficult-to-work-with systems, a minimalist approach is recommended for pilot studies. Once a baseline functional CRISPR protocol is established, modifications can be made to make the CRISPR system more robust. While the first cut can be arduous, the capacity for applications of Cas9 are well worth the effort. To help design a CRISPR construct for use in non-model systems, the following checklist can be used as a roadmap for all the necessary components (Figure C5-10). While CRISPR Cas9 opens the door to genetic modification of many recalcitrant organisms, acting as a multitool for organisms with comparatively small genetic toolboxes, implementation of the system can be difficult. Many of the barriers to implementing the systems do not lie in the capacity of CRISPR Cas9 itself, but rather the delivery methods available to novel organisms. 


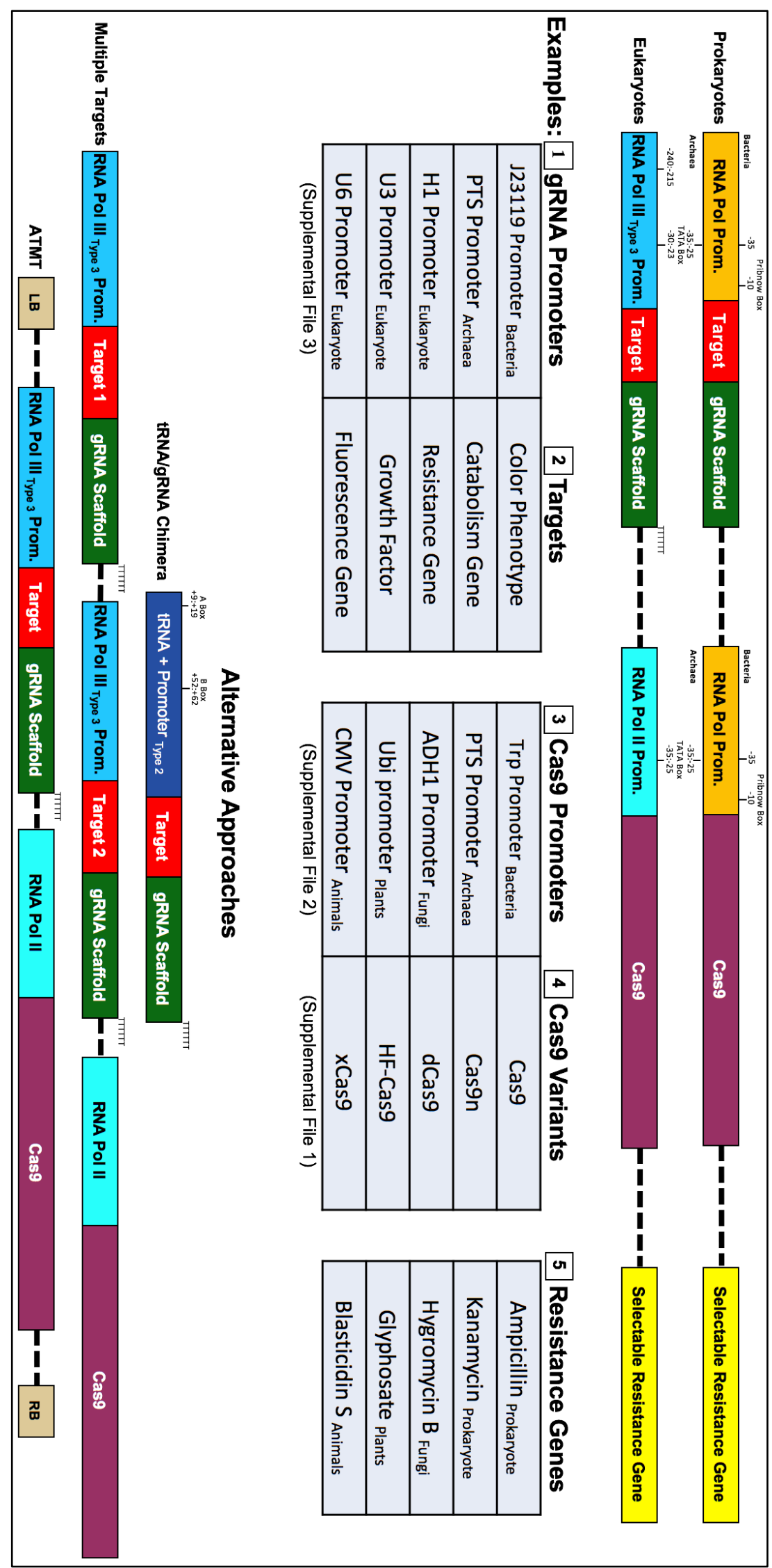


Figure C5-10 Checklist, with examples, of components needed to generate a CRISPR Cas9 construct for use in a new system. Sequences for the example promoters and Cas9 variants can be found in supplemental files 1-3. Not shown are other species-specific RNA Pol II promoters used successfully to drive Cas9 expression in fungi (Ustilago maydis, phsp 70, potef; [Shuster et al., 2018]), protozoans (Toxoplasma gondii, TUB1 promoter and SAG1 3' UTR; [Sidik et al., 2018]), insects (Drosophila, hsp70Bb; [Taning et al., 2017]), and zebrafish (tissue-specific promoters; [Albain et al., 2015; Hwang et al., 2013; Li et al., 2016]).

\subsection{Implementing CRISPR Cas9 in the Microbotryum genus}

The ability to manipulate an organism's primary genetic material depends heavily on the tools available to each system. Every established transformation approach can therefore be considered a new tool in an organism's "toolbox", the size of which varies among systems depending heavily on the difficulty of introducing material into cells and how the organism deals with the foreign material. Organisms that readily take up foreign DNA during transformation often draw the attention of researchers as easy to work with systems, and in turn the increase in their use results in the broadening of transformation approaches. Some of the most well studies organisms, e.g. Drosophila melanogaster, Saccharomyces cerevisiae, and Arabidopsis thaliana, are therefore the metaphorical "kings" of their respective kingdoms due to their ease of use, breadth of study, and consequently their comparatively large toolboxes.

Because of their extensive repertoire of approaches for molecular genetic analyses, model organisms are often used as proxies for studying genes from more recalcitrant organisms; however, studies that examine the unique relationships between species, e.g., exquisitely specific host-pathogen interactions, require at least a fundamental ability to generate knockouts in these species. Unique genomic milieus, reproductive cycles, co-evolution, or natural environmental factors are all variables that can have major effects on the ability of scientists to manipulate, species. The fungal pathogen species complex Microbotryum violaceum is one such recalcitrant group of organisms used to study the life cycle of dimorphic pathogens, the evolution of disease, and the emergence of host shifts (Schäfer et al., 2010; Beckerson et al., 2019); however, the M. violaceum species complex is difficult to transform and has a comparatively small molecular genetic toolbox.

While classical genetic approaches have been implemented in Microbotryum with widespread success, manipulating the primary genetic material of these fungi has proven difficult. Despite these difficulties, one 
recent breakthrough study has demonstrated that Agrobacterium-mediated transformation (ATMT) can be utilized to randomly insert genetic material into transcriptionally active portions of the fungal genome (Toh et al., 2016), allowing for transgenic expression of genes in the Microbotryum species complex. However, although a reliable ATMT method has opened the door for over expression and transgenic expression of genetic material in this system, a means of site-specific gene targeting and knockouts have, until now, remained a major hurdle.

To establish CRISPR-Cas9 in Microbotryum, our group used a three-pronged approach of 1) modifying a Ustilago maydis CRISPR-Cas9 plasmid for delivery into Microbotryum via electroporation, 2) building upon the previously established ATMT approach to deliver the CRISPR-Cas9 components, and 3) utilizing a kit designed to create the Cas9-gRNA duplex in vitro and deliver it into the cells using PEG treatment of protoplasted cells.

\section{Materials and Methods}

\subsection{Selecting a target}

As a pilot study for demonstrating a successful knockout using CRISPR-Cas9 in the Microbotryum species complex, our group attempted to create an easily observable color phenotype change from the bright pink color of M. lychnidis-dioicae cells in their saprophytic yeast-like life stage by targeting the coding region for a putative oxidoreductase protein, MVLG_05585, with predicted activity in the carotene biosynthesis pathway (Garber and Day, 1985). To minimize off-target Cas9 activity, a high-fidelity variant of Cas9 (Cas9HF) was used for the ATMT approach and target sequences least likely to cause off target cuts were selected for MVLG_05585 following guidelines in the CRISPR-Cas9 review published by Hsu, Lander, and Zhang (Hsu et al., 20174). Target sequences that met these criteria were used in Blastn searches against the available Microbotryum lychnidis-dioicea genome available at the Joint Genome Institute (JGI) Genome Portal (https://mycocosm.jgi.doe.gov/Micld1/Micld1.home.html), and any target with more than 1 hit was removed.

For the ATMT method, a single target was selected within the first exon of the MVLG_05585 gene. For the PEG treatment approach, two targets were selected, one at the 5' beginning of the MVLG_05585 gene and 
the other at the 3' end of the MVLG_05585 gene. These targets were used simultaneously with the Cas9 enzyme in attempt to completely excise the gene to ensure a knockout.

\subsection{Constructing a plasmid for delivery of CRISPR-Cas9 via electroporation}

The single plasmid construct for expression of a sgRNA and Cas9 in Microbotryum, pMvCC9, was created via modification of pMS10, provided by Dr. Regine Kahmann, which included the sequence for the Cas9 endonuclease optimized for Ustilago maydis expression, an poly A termination sequence, as well as an sgRNA cassette driven by the universal U6 Polymerase III promoter, along with an RNA Pol III terminator. To adapt this plasmid for use in Microbotryum, the U6 promoter was replaced with a tRNA/sgRNA chimera sequence (Wei et al., 2015) to drive expression of the sgRNA, for which the target sequence was replaced with 19 base pairs to target the MVLG_05585 gene. The Polymerase II promoter from the constitutively expressed MVLG_02523 gene, defined as the $1 \mathrm{~kb}$ region upstream of the start codon, was also cloned in place of the Potef promoter from pMS10 to drive expression of the Cas9 endonuclease in Microbotryum (Figure C5-11). Furthermore, to select for transformants, the Hygromycin B resistance cassette driven by the MVLG_05589 promoter from the Microbotryum Agrobacterium-mediated transformation plasmid, pMvHyg (Toh et al., 2016), was cloned in-between the sequence for Carboxin resistance present in pMS10 to allow for selection of Microbotryum cells on Hygromycin containing media and for quick excision if the plasmid need be reverted to a function Carboxin resistance gene (Figure C5-11). 


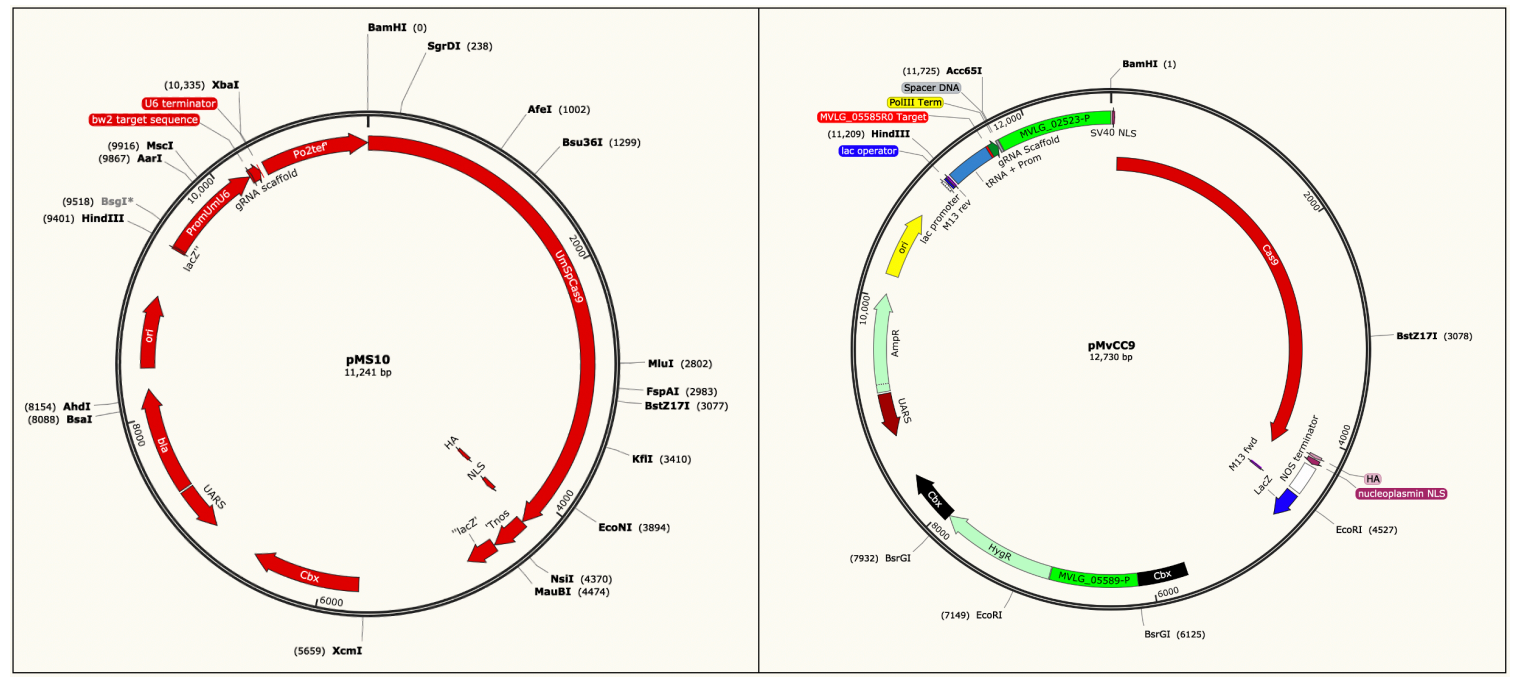

Figure C5-11 Plasmid maps for pMs10 and pMvCC9. The pMS10 plasmid is shown on the left, while the pMvCC9 plasmid which incorporates various components of pMS10 is shown on the right.

These substitutions were made using the restriction endonuclease cut sites available in the pMS10 vector, HindIII and Acc65I for the insertion of the tRNA/gRNA chimera sequence, and Acc65I and BamHI for insertion of the MVLG_02523 promoter sequence, followed by ligation and transformation of the modified plasmid into E. coli.

\subsection{Electroporation of Microbotryum lychnidis-dioicae cells}

An assay of various electroporation conditions was used to determine optimal transformation efficiency of Microbotryum lychnidis-dioicae cells using a Bulldog Bio@ / Nepa Gene ELEP021 Electroporator. 2 mm gap cuvettes were used to electroporate M. lychnidis-dioicae cells suspended at an $\mathrm{OD}_{600}=1$ in sorbitol as a buffer. Two variables were assayed for the poring pulse, the voltage (V) and length of pulse in ms. The interval, number of pulses, and polarity for the poring pulse remained constant at $50 \mathrm{~ms}, 1$ pulse, and positive polarity. Conditions were also kept constant for the transfer pulse voltage, length, interval, number of pulses, and polarity at $50 \mathrm{~V}, 50 \mathrm{~ms}, 50 \mathrm{~ms}, 5$ pulses, and alternating positive/negative polarity. The ohms and volts, as well as the amps, and joules output for both the poring and transfer pulses, were recorded in each trial. Cells were then removed from the electroporator and immediately plated onto YPD-10\% growth agar. Colonies were then counted to determine optimal survival rate with the highest shock values. 
After conditions for optimal survivorship were determined, electroporation was repeated in attempt to transform M. lychnidis-dioicae cells with pMvCC9. Cells were immediately suspended in YPD-10\% growth media and shaken at $27^{\circ} \mathrm{C}$ for 2 hours before being plated onto YPD- $10 \%$ containing $150 \mu \mathrm{g} / \mathrm{mL}$ Hygromycin as a selective agar media.

Table C5-2. Optimal electroporation settings assay.

\begin{tabular}{|c|c|c|c|c|c|c|c|c|c|c|}
\hline \multirow[b]{3}{*}{ \# } & \multicolumn{10}{|c|}{ Set Parameters } \\
\hline & \multicolumn{5}{|c|}{ Poring Pulse } & \multicolumn{5}{|c|}{ Transfer Pulse } \\
\hline & $\mathrm{V}$ & $\begin{array}{c}\text { Length } \\
(\mathrm{ms})\end{array}$ & $\begin{array}{c}\begin{array}{c}\text { Interval } \\
(\mathrm{ms})\end{array} \\
\end{array}$ & No. & Polarity & $\mathrm{V}$ & $\begin{array}{c}\text { Length } \\
(\mathrm{ms})\end{array}$ & $\begin{array}{c}\text { Interval } \\
(\mathrm{ms}) \\
\end{array}$ & No. & Polarity \\
\hline 1 & Contr & ال (cell & and $\mathrm{C}$ & NA & vithout & elec & tropor & tion $\mathrm{tr}$ & atn & ent) \\
\hline 2 & 2000 & 1 & 50 & 1 & + & 50 & 50 & 50 & 5 & $+/-$ \\
\hline 3 & 2000 & 2 & 50 & 1 & + & 50 & 50 & 50 & 5 & $+/-$ \\
\hline 4 & 2000 & 3 & 50 & 1 & + & 50 & 50 & 50 & 5 & $+/-$ \\
\hline 5 & 2000 & 4 & 50 & 1 & + & 50 & 50 & 50 & 5 & $+/-$ \\
\hline 6 & 2000 & 5 & 50 & 1 & + & 50 & 50 & 50 & 5 & $+/-$ \\
\hline 7 & 2500 & 1 & 50 & 1 & + & 50 & 50 & 50 & 5 & $+/-$ \\
\hline 8 & 2500 & 2 & 50 & 1 & + & 50 & 50 & 50 & 5 & $+/-$ \\
\hline 9 & 2500 & 3 & 50 & 1 & + & 50 & 50 & 50 & 5 & $+/-$ \\
\hline 10 & 2500 & 4 & 50 & 1 & + & 50 & 50 & 50 & 5 & $+/-$ \\
\hline 11 & 2500 & 5 & 50 & 1 & + & 50 & 50 & 50 & 5 & $+/-$ \\
\hline 12 & 3000 & 1 & 50 & 1 & + & 50 & 50 & 50 & 5 & $+/-$ \\
\hline 13 & 3000 & 2 & 50 & 1 & + & 50 & 50 & 50 & 5 & $+/-$ \\
\hline 14 & 3000 & 4 & 50 & 1 & + & 50 & 50 & 50 & 5 & $+/-$ \\
\hline
\end{tabular}

\subsection{Constructing a plasmid for ATMT delivery of CRISPR-Cas9}

The CRISPR-Cas9 ATMT plasmid for use in Agrobacterium-mediated Transformation (ATMT) of $M$. violaceum species (pMvHyg_CRISPR) was created using scaffold from pMS8 (provided by Dr. Regine Kahmann) as a scaffold. The pMS8 plasmid orginally introduced the Cas9 gene from $S$. pyogenes to the Ustilago maydis transformation plasmid pNEBUC (Schuster, 2015). The U6 promoter attached to the sgRNA scaffold in pMS8 was replaced with a tRNA/sgRNA chimera sequence (Wei et al., 2017) generated using the TyrGTA tRNA from M. lychnidis-dioicae, including its promoter to drive Polymerase III transcription. TyrGTA was chosen due to demonstrated codon bias in M. lychnidis-dioicae (Perlin et al., 2015). The replacement of the U6 promoter with the tRNA/sgRNA chimera was performed via Gibson overlap extension 
PCR utilizing the NEBuilder ${ }^{\circledR}$ \#E2621 HiFi DNA Assembly Master Mix Kit to connect the TyrGTA tRNA with the MVLG_05585 target sequence, the gRNA scaffold from pMS8, and a tRNA transcriptional termination signal along with DNA spacer shown in Figure C5-11.

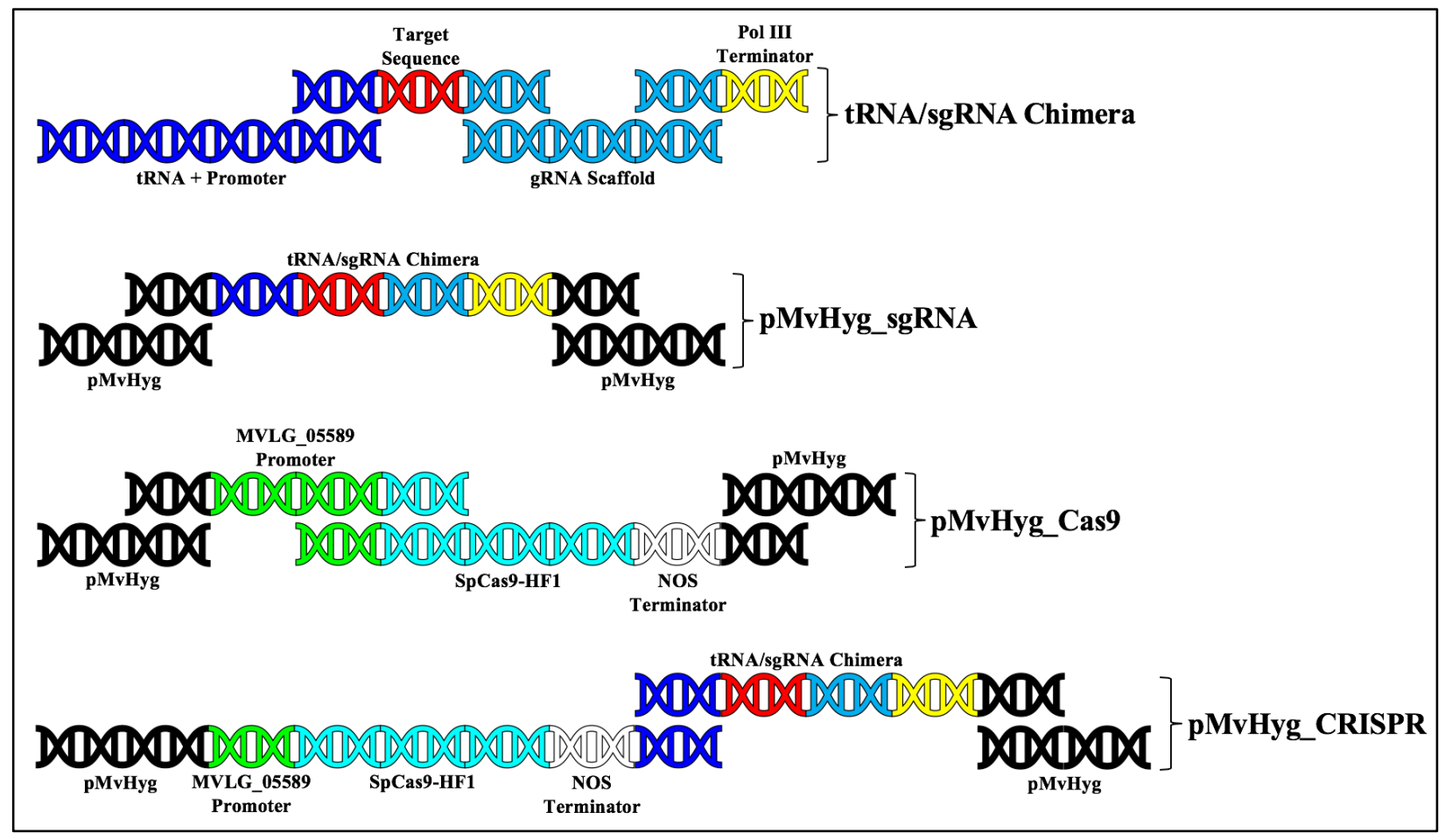

Figure C5-12 Stepwise overview of pMvHyg_CRISPR construction via Gibson Overlap PCR. Overlap PCR using double stranded DNA fragments with $20 \mathrm{bp}$ complementary sequence to adjacent fragments.

The gRNA and the termination signal fragments were ordered as $60 \mathrm{bp}$ oligonucleotides with $20 \mathrm{bp}$ of overlap regions for Gibson overlap PCR. These oligonucleotides were converted into double stranded DNA by adding equimolar concentrations of each oligo and its corresponding complement oligo to a PCR tube and heating in a thermocycler at $94^{\circ} \mathrm{C}$ for $1 \mathrm{~min}$ to denature any secondary structures, followed by $60^{\circ} \mathrm{C}$ for 10 minutes to allow for annealing of the two complements. The tRNA sequence including the promoter were amplified from M. lychnidis dioicae genomic DNA, and gRNA scaffold was amplified from pMS8 via PCR. The primers used to amplify these two fragments also included a 5' $20 \mathrm{bp}$ sequence overlap for Gibson overlap PCR (Figure 1). The 4 fragments were annealed using NEBuilder ${ }^{\circledR}$ \#E2621 HiFi DNA Assembly Master Mix Kit, followed by another round of PCR using Ex Taq polymerase with a forward primer for the tRNA with a 
$20 \mathrm{bp}$ complementary sequence to the pMvHyg XbaI restriction site, and a reverse primer for the 3' end of the fragment including a $20 \mathrm{bp}$ complementary sequence for the StuI restriction sequence of pMvHyg (Figure 1). This generated a single fragment with $\mathrm{XbaI}$ and StuI cut sites as well as the overhangs necessary for Gibson overlap PCR. A double restriction digest was performed on the pMvHyg vector with XbaI and StuI, and both the vector and PCR product were separated by gel electrophoresis through a $0.8 \%$ agarose gel containing ethidium bromide alongside a DNA size standard via gel electrophoresis. The corresponding bands for the digested pMvHyg vector and tRNA/sgRNA fragment were excised from the gel, purified, and annealed using the NEBuilder ${ }^{\circledR}$ \#E2621 HiFi DNA Assembly Master Mix Kit to create the vector pMvHyg_sgRNA (Figure 1)

A separate ATMT vector containing a high-fidelity mutant of the Cas9 gene (pMvHyg_Cas9) was also generated using Gibson overlap PCR. The coding region for the high fidelity Cas9 variant (also containing an NLS) was amplified using pCas9hf, obtained from Dr. Björn Sandrock. This high-fidelity Cas9 endonuclease was created using three missense substitutions; aa650 Arg->Ala, aa684 Gln->Ala, and aa915 Gln->Ala, and has been demonstrated to create fewer off-target cuts. To drive expression of this Cas9-HF endonuclease, the Po2tef promoter from the vector was replaced with a constitutively expressed promoter from M. lychnidis-dioicae gene, MVLG_05585. The MVLG_05589 promoter sequence was amplified from M. lychnidis-dioicae genomic DNA using 40 bp primers which contained 20 bp complementary sequence to the elements upstream of the PacI cut site on pMvHyg and Cas9-HF. The Cas9-HF coding region, including the NOS Termination sequence was amplified using similar 40 bp primers with 20 bp complementary sequence for the MVLG_05589 promoter and the elements downstream of the XbaI cut site in pMvHyg. These 2 fragments were then cloned into cut pMvHyg vector using the NEBuilder ${ }^{\circledR}$ \#E2621 HiFi DNA Assembly Master Mix Kit to generate pMvHyg Cas9 (Figure C5-11).

To create the single transfer cassette containing both the tRNA/sgRNA chimera and the Cas9-HF, the tRNA/sgRNA fragment from pMvHyg_tRNA/sgRNA vector was amplified using $40 \mathrm{bp}$ primers including 20bp overlap regions for the elements upstream and downstream of the XbaI and StuI cut sites, including the sequence to retain the cut sites themselves for future modifications, and the pMvHyg_Cas9 vector was double digested with XbaI and StuI. The fragment was then cloned into the cut vector using the 
the NEBuilder ${ }^{\circledR}$ \#E2621 HiFi DNA Assembly Master Mix Kit to form the pMvHyg_CRISPR Plasmid (Figure C5-11 and C5-12).

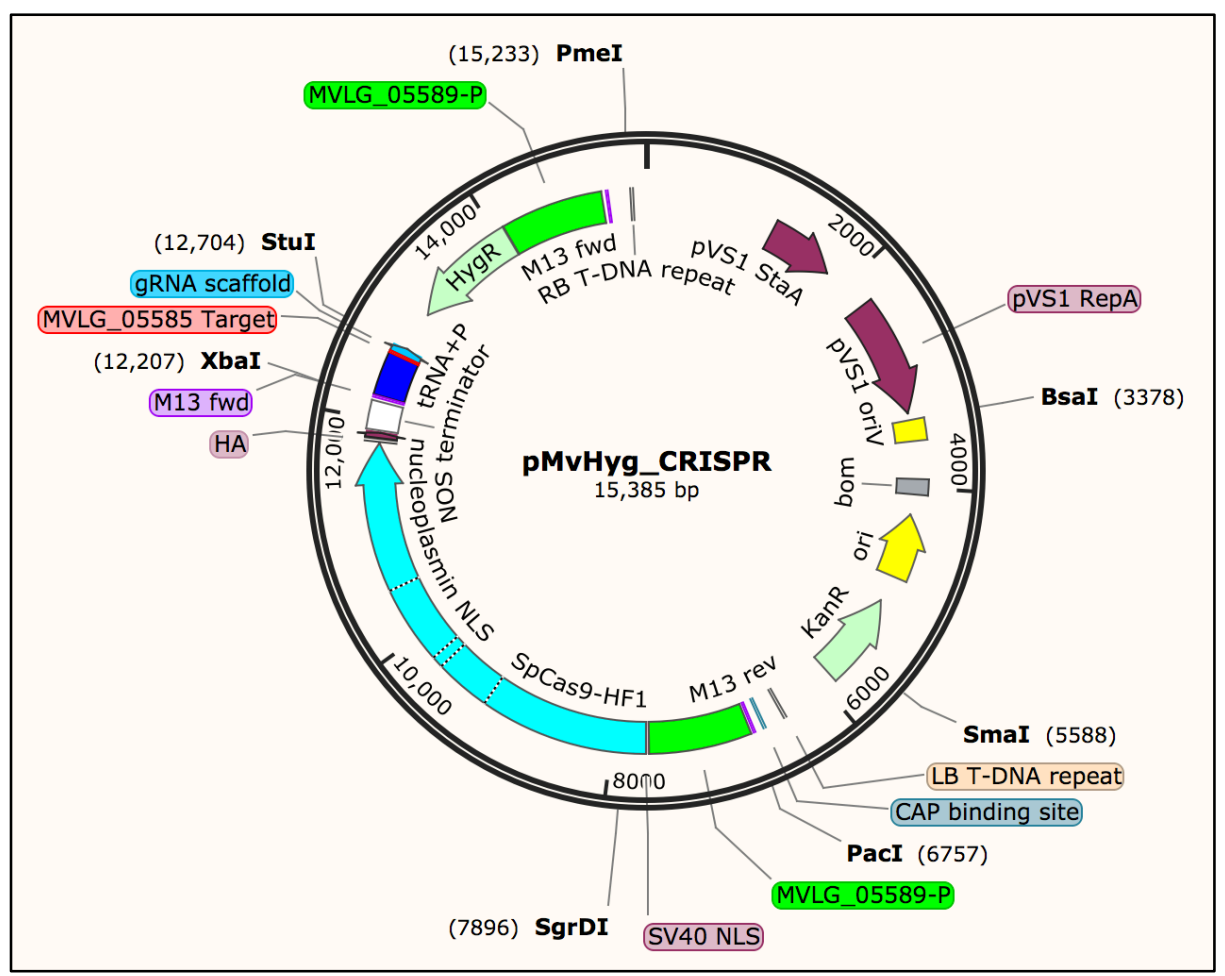

Figure C5-13 Snapgene image of the pMvHyg_CRISPR plasmid. The plasmid map for the ATMT vector containing the Cas9-HF gene driven by the constitutively expressed MVLG_05589 promoter, and the tRNA/gRNA chimera targeting the MVLG_05585 $\beta$-carotene synthesis gene.

When used in ATMT, the cassette cloned in-between the T-DNA Left Border and Right Border is incorporated randomly into transcriptionally active regions of the host genome. 


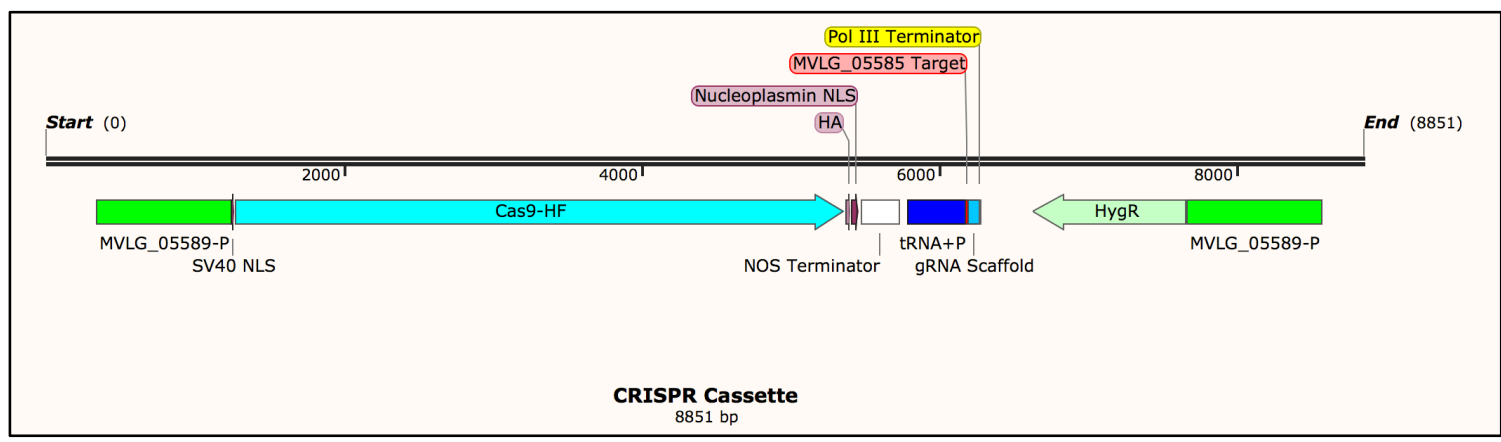

Figure C5-14 Snapgene image for the components of pMvHyg_CRISPR that are transferred by the

left and right T-DNA borders. The T-DNA cassette that is excised from the ATMT vector and incorporated randomly into transcriptionally active areas of the host (Microbotryum lychnidis-dioicae) genome.

\subsection{Agrobacterium-mediated transformation of M. lychnidis-dioicae cells with pMvHyg-CRISPR}

pMvHyg_CRISPR was transformed into EHA105 Agrobacterium cells using electroporation $(2.5 \mathrm{kV}, 400$ ohms and $25 \mu \mathrm{F}$ ) following the protocol outlined in Toh et al., (2016). Transformants were verified via streaking colonies onto LB agar-containing $50 \mu \mathrm{g}$ kanamycin $/ \mathrm{mL}$. Putative transformants were then restreaked onto LB containing $50 \mu \mathrm{g}$ kanamycin and $100 \mu \mathrm{g}$ spectinomycin per $\mathrm{mL}$ agar to ensure that both the pMvHyg containing the respective species-specific gene and the helper plasmids were in the cells. Surviving EHA105 cells were further confirmed as bearing the desired constructs through colony PCR, before being used to transform p1A1 mating type cells of M. lychnidis-dioicae. For these experiments, 10E7 of Microbotryum cells and 10E7 Agrobacterium cells were used, as measured spectrophotometrically (Microbotryum: $\mathrm{OD}_{600} 1=3.4 \mathrm{E} 7$ cells $/ \mathrm{mL} ;$ Agrobacterium: $\mathrm{OD}_{600} 1=8 \mathrm{E} 8 \mathrm{cells} / \mathrm{mL}$ ) and mixed in equal volumes. $200 \mu \mathrm{L}$ of each suspension were spotted onto IM plates containing acetosyringone. Spotted plates were let sit at room temperature, $\sim 25^{\circ} \mathrm{C}$, for 3 days, after which the resulting mass of cells was scraped from the plates, suspended in $600 \mu \mathrm{L}$ of YPD-10\% broth, and $200 \mu \mathrm{L}$ of each suspension were spread onto YPD$10 \%$ containing $150 \mu \mathrm{g} / \mathrm{mL}$ Hygromycin and $100 \mu \mathrm{g} / \mathrm{mL}$ Cefotaxime plates. Each plate was then incubated for 12-15 days to select for transformed Microbotryum cells. Colonies appearing were streaked onto fresh YPD-10\% containing $150 \mu \mathrm{g} / \mathrm{mL}$ Hygromycin B plates to ensure proper transformation and later verified for successful CRISPR cassette insertions via sequencing. 


\subsection{Protoplasting Microbotryum cells}

Microbotryum cells were protoplasted using a protocol developed by Dr. Naoko Fujita. The enzyme solution was prepared using $2 \%$ lysing enzymes from Trichoderma harzianum and $2 \%$ Driselase in $1 \mathrm{M} \mathrm{MgSO}_{4}$. The solution was mixed and stored overnight at $4^{\circ} \mathrm{C}$, after which the tube was spun at $11,000 \mathrm{rpm}$ for 10 minutes. The supernatant was filtered into a sterile $50 \mathrm{~mL}$ Falcon Tube using filters. To protoplast M. lychnidis-dioicae cells, p1A1 and p1a2 strains were grown on YPD with 10\% dextrose (YPD-10\%) agar plates for 2 days at $27^{\circ} \mathrm{C}$. A generous loop of cells was suspended in $5 \mathrm{~mL}$ of the filtered enzyme solution in a $50 \mathrm{~mL}$ sterile tube and shaken using a platform shaker at medium-low speed overnight. Parafilm was placed around the lid to prevent any leakage. The following morning, $5 \mathrm{~mL}$ of $1.2 \mathrm{M}$ mannitol was carefully added top wise so as not to mix with the cell suspension. The layered mixture was then carefully spun at 2,000 rpm for 20 minutes. After 20 minutes, a layer of protoplasted cells formed between the cell suspension bottom layer and mannitol top layer. This middle layer was extracted using a P1000 micropipette tip. Of the $20 \mathrm{~mL}$ solution, approximately $5 \mathrm{~mL}$ of protoplasts were extracted. This layer of protoplasted cells were confirmed using compound microscopy and aliquoted into $100 \mu \mathrm{L}$ aliquots in $600 \mu \mathrm{L}$ microcentrifuge tubes. Aliquots were centrifuged at 14,000 rpm for $1 \mathrm{~min}$, the supernatant was removed, and the protoplasted cells were resuspended in $100 \mu \mathrm{L}$ of STC before storage long-term at $-80^{\circ} \mathrm{C}$. Protoplasted stored at $-80^{\circ} \mathrm{C}$ were tested for viability on YPD-10\% and demonstrated the capacity to regenerate their cell walls and grow even after 2 years of storage. 


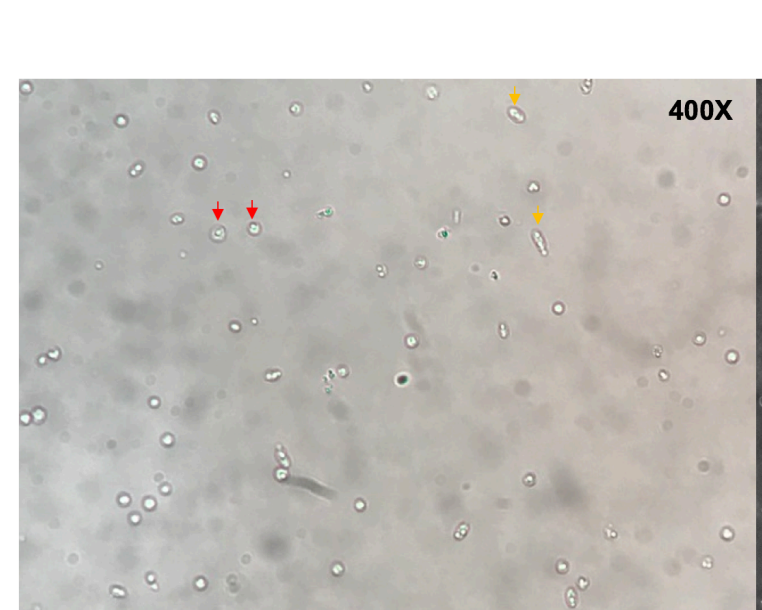

Photomicrograph

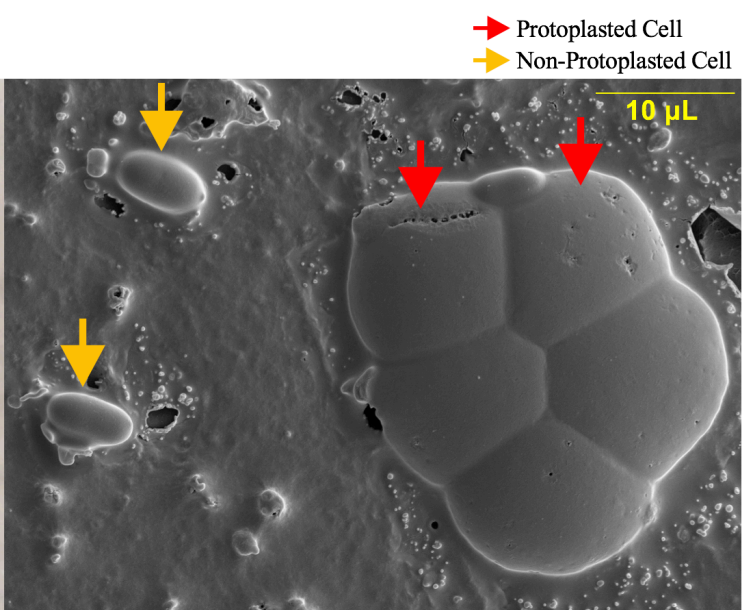

Electron Micrograph

Figure C5-15 Microscopy images of protoplasted Microbotryum cells. Photomicrograph of spheroplasted (red arrow) and normal (orange arrow) Microbotryum lychnidis-dioicae cells, left, and electron micrograph of spheroplasted and normal M. lychnidis-dioicae cells, right.

\subsection{PEG Transformation of Microbotryum with in vitro Cas9 Duplex}

In vitro duplexing of S.p. Cas9 Nuclease was accomplished following the protocol for the IDT Alt-R ${ }^{\mathrm{TM}}$ kit. gRNAs duplexed with Cas9 were assembled from custom crRNAs annealed to Alt- ${ }^{\mathrm{TM}}$ tracrRNAs by mixing the two at equimolar concentrations using $5 \mu \mathrm{L}$ of each $100 \mu \mathrm{M}$ working stock and $5 \mu \mathrm{L}$ of Nuclease-Free Duplex Buffer provided with the Alt- $\mathrm{R}^{\mathrm{TM}}$ tracrRNAs, and incubating the mixture at $95^{\circ} \mathrm{C}$ for 5 minutes before allowing the tubes to cool to room temperature. To increase transformation efficiency, two gRNAs were designed for each knockout, one targeting an NGG PAM site at the 5' end of the gene and the other targeting an NGG PAM site at the 3' end of the gene, allowing for entire gene deletion and insertion of a selectable marker-encoding linear oligonucleotide with $40 \mathrm{bp}$ overlap regions corresponding to the upstream and downstream regions of the excised target. Therefore, to direct Cas9 activity at both ends of the target gene, the Alt-R ${ }^{\mathrm{TM}}$ S.p. Cas9 Nuclease 3NLS was diluted $10 \mathrm{x}$ using $1 \mu \mathrm{L}$ of the Cas 9 and $9 \mu \mathrm{L}$ of Cas 9 Working Buffer (20 mM HEPES pH 7.5, $150 \mathrm{mM} \mathrm{KCl}$ in Nuclease-free water) and $1.5 \mu \mathrm{L}$ of the enzyme solution was mixed with $1.5 \mu \mathrm{L}$ of the 5' target gRNA and 1.5 of the $\mu \mathrm{L}$ 3' gRNA target in $22 \mu \mathrm{L}$ of Cas9 Working Buffer. The duplex solution was incubated at room temperature for 5 minutes to allow for annealing of the Cas 9 to gRNAs before use in PEG transformation. 
M. lychnidis-dioicae protoplasts were retrieved from $-80^{\circ} \mathrm{C}$ and thawed on ice. $100 \mu \mathrm{L}$ of STC solution was added to an $8 \mathrm{~mL}$ snap cap tube and placed on ice. $7 \mu \mathrm{g}$ of purified linear repair template containing the MVLG_05589 promoter and coding region for Hygromycin-B resistance in M. lychnidisdioicae (amplified from the pMvHyg vector) was added to the STC solution along with the $26.5 \mu \mathrm{L}$ of RNP duplex solution. $100 \mu \mathrm{L}$ of protoplasts were then added to the tubes using wide-bore tips and gently mixed with the pipette to ensure even suspension without destroying the protoplasted cells. $50 \mu \mathrm{L}$ of $30 \%$ PEG solution was added to the mixture and gently swirled before further incubation on ice for $50 \mathrm{~min}$. After incubation on ice, the tube was placed at room temperature and $2 \mathrm{~mL}$ of $30 \%$ PEG was added to the solution and mixed via gentle repeated inversion. The tube was incubated at room temperature for $20 \mathrm{~min}$. Protoplasted cells become clumped together during this time and were examined using a compound microscope. After verification of clumping, $2 \mathrm{~mL}$ of STC was added to the suspension and mixed via inversion. $500 \mu \mathrm{L}$ of suspension was then top added to the top agar YPD-10\% plates. Top agar plates were prepared by pouring $10 \mathrm{~mL}$ of YPD-10\% media with $300 \mu \mathrm{g} / \mathrm{mL}$ Hygromycin-B, allowing the agar to solidify, and then adding $10 \mathrm{~mL}$ of YPD-10\% non-drug top agar during the $20 \mathrm{~min}$ incubation step above. To increase efficiency, the bottom agar was prepared the morning of transformation. Plates were allowed to dry before storage at $25^{\circ} \mathrm{C}$ for two weeks. After 11 days, small colonies began to appear on the plates. More colonies appeared between days 12-16, and the original colonies were large enough to pick and streak onto a fresh YPD- $10 \%$ containing $300 \mu \mathrm{g} / \mathrm{mL}$ Hygromycin-B plate by day 13 . The newly streaked plates were incubated at $25^{\circ} \mathrm{C}$ for 3 more days before colonies with turbid growth, indicating true resistance to the Hybromycin-B, were selected and added to liquid YPD-10\% containing $200 \mu \mathrm{g} / \mathrm{mL}$ Ampicillin tubes. These tubes were spun in an orbital shaker at a medium speed for 2 days before DNA extractions were performed and PCR products were sent for sequencing.

\subsection{Sequencing to confirm successful knockouts}

Primers for amplification of the target gene for PCR confirmation and sequencing were designed to begin 120 bp upstream of the 5' cut site and 120 bp downstream of the 3' cut site. PCR using Ex Taq DNA pol was performed on genomic DNA extracts from potential transformants and separated on an agarose gel to screen for insertion of the $\mathrm{Hyg}^{\mathrm{R}}$ cassette. The PCR product for wildtype, untransformed cells is $2,351 \mathrm{bp}$ for the 
MVLG_05585 target, and 1,024 bp for the MvS1_01693 target. If the Hyg ${ }^{\mathrm{R}}$ cassette is successfully inserted in place of the excised gene, the band size for both sets of primers should be approximately 2,140 bp. Bands that appeared at the appropriate sizes were purified using the Zymoclean ${ }^{\mathrm{TM}}$ Gel DNA Recovery Kit and sent for sequencing using the 5 ' end forward primers.

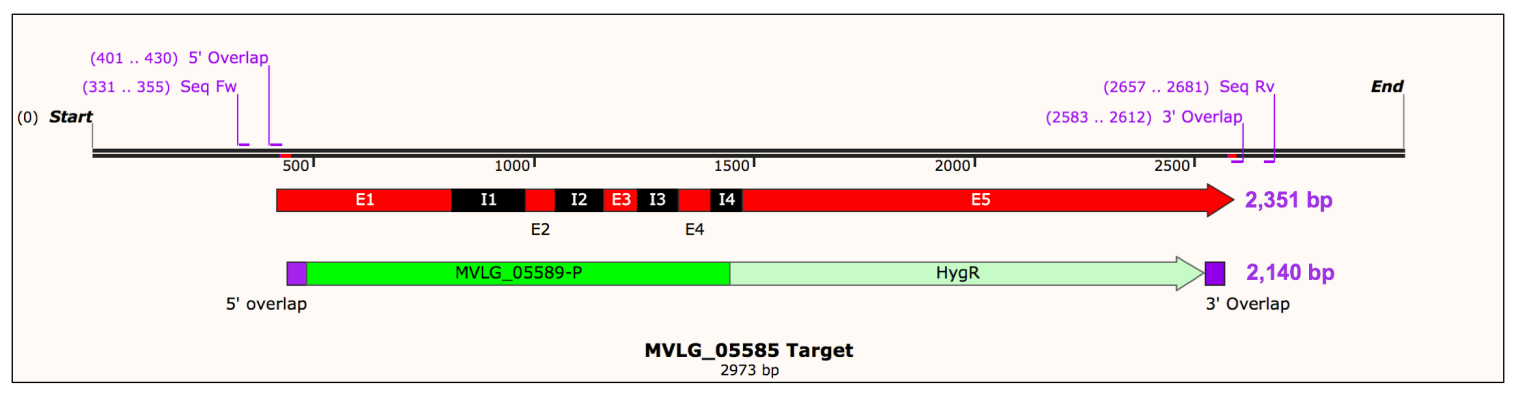

Figure C5-16 Snapgene image depicting the target regions and insertion construct for MVLG_05585

knockouts. MVLG_05585 gene, top, and $\mathrm{Hyg}^{\mathrm{R}}$ cassette insert, bottom. Purple text on top of the DNA sequence indicates the location of the sequencing primers (Seq Fw and Seq Rv) 120 bp upstream and downstream of the 5' and 3' cut sites used for confirming knockouts, and the overlap locations for homologydirected repair utilizing the $\mathrm{Hyg}^{\mathrm{R}}$ cassette insert. Thin red DNA bars indicate the target sequence, both on the reverse strand of MVLG_05585. Red feature boxes labeled E\# indicate exonic regions of the gene while black feature boxes labeled I\# indicate intronic regions of the gene. The purple feature boxes on the $\mathrm{Hyg}^{\mathrm{R}}$ cassette insert indicate the 40 bp overlap sequences for use as homology-directed repair template. The bold purple text next to each set of features indicates the size of the PCR product when using the Seq Fw and Seq Rv primers.

\section{Results}

\subsection{Electroporation of Single Plasmid CRISPR Construct}

Optimal electroporation conditions for cell viability after the procedure were determined by observing the cultures plated from each of the trials in the electroporation assay. The control (untreated) demonstrated confluent growth, as did treatment conditions with 1-2 ms of $2000 \mathrm{~V}$. Treatment with 3-4 ms of $2000 \mathrm{~V}$ reduced the survival rate of cells, resulting in cultures that had $>200$ colonies per plate, but not confluent 
growth. Treatment conditions of $2000 \mathrm{~V}$ for $5 \mathrm{~ms}$, as well as $2500 \mathrm{~V}$ for $1-5 \mathrm{~ms}$ resulted in a significant decrease in survivability for cells. All trials using $3000 \mathrm{~V}$ of electricity resulted in no growth on culture plates.

Table C5-3. Electroporation results

\begin{tabular}{|c|c|c|c|c|c|c|c|}
\hline \multirow[b]{3}{*}{$\#$} & \multicolumn{5}{|c|}{ Measurements } & \multicolumn{2}{|r|}{ Results } \\
\hline & \multirow[b]{2}{*}{$\mathrm{k} \Omega$} & \multicolumn{2}{|c|}{ Poring Pulse } & \multicolumn{2}{|c|}{$\begin{array}{l}\text { Transfer } \\
\text { Pulse } \\
\end{array}$} & \multirow{2}{*}{$\begin{array}{c}\text { Voltage } \\
\text { (V) }\end{array}$} & \multirow[b]{2}{*}{ Comments } \\
\hline & & A & J & A & $J$ & & \\
\hline 1 & & & & & & & Confluent Growth \\
\hline 2 & 5.308 & 0.39 & 1.16 & 0.04 & 4.71 & 2000 & Confluent Growth \\
\hline 3 & 5.129 & 0.40 & 2.62 & 0.05 & 5.64 & 2000 & Confluent Growth \\
\hline 4 & 5.750 & 0.35 & 3.70 & 0.05 & 5.25 & 2001 & Lots of Colonies \\
\hline 5 & 5.099 & 0.39 & 6.06 & 0.07 & 6.44 & 2001 & $\begin{array}{l}\text { Lots of Colonies } \\
\text { *optimal conditions }\end{array}$ \\
\hline 6 & 5.722 & 0.35 & 6.63 & 0.06 & 5.61 & 2001 & Few Colonies \\
\hline 7 & 4.981 & 0.53 & 1.96 & 0.05 & 5.45 & 2495 & Few Colonies \\
\hline 8 & 4.666 & 0.55 & 4.77 & 0.07 & 6.49 & 2499 & Few Colonies \\
\hline 9 & 4.668 & 0.55 & 8.17 & 0.08 & 7.44 & 2499 & Few Colonies \\
\hline 10 & 4.868 & 0.83 & 17.70 & 0.14 & 10.22 & 2490 & Few Colonies \\
\hline 11 & 3.883 & 0.66 & 22.40 & 0.17 & 11.70 & 2500 & Few Colonies \\
\hline 12 & 4.303 & 0.73 & 3.25 & 0.07 & 6.50 & 2966 & No Growth \\
\hline 13 & 4.083 & 0.77 & 8.96 & 0.10 & 9.16 & 2986 & No Growth \\
\hline 14 & 3.993 & 0.79 & 29.36 & 0.01 & 2.21 & 2932 & No Growth \\
\hline
\end{tabular}

Results from this preliminary survivorship assay indicates that the settings of $2000 \mathrm{~V}$ for $4 \mathrm{~ms}$ is likely to result in the highest transformation efficiency for this system.

Using these settings, our we were able to verify that dyed molecules can be transformed into $M$. lychnidis-dioicae cells; however, transformation attempts with the pMvCC9 plasmid resulted in no colonies on selective media, indicating that while transfer of material into the cells is possible with electroporation problems with either the plasmid construct or its entry into the nuclease prevent the CRISPR components from being expressed via the single plasmid method.

\subsection{Agrobacterium-mediated Transformation of M. lychnidis-dioicae with pMvHyg_CRISPR}

Agrobacterium-medicated transformation of Microbotryum cells with the pMvHyg_CRISPR plasmid resulted in 5 colonies that were verified to contain the components between the left and right transfer borders 
via PCR. Despite successful insertion of the both the tRNA/gRNA chimera and Cas9 coding regions, none of the 5 cell lines demonstrated any modification to the target region of MVLG_05585. Furthermore, cell color in each line remained the wild-type pink. However, despite an inability to knockout the MVLG_05585 gene, qrtPCR analysis of the transformed Microbotryum cells indicates that the pMvHyg_CRISPR plasmid does successfully induce the production of the Cas9 (Figure C5-17).

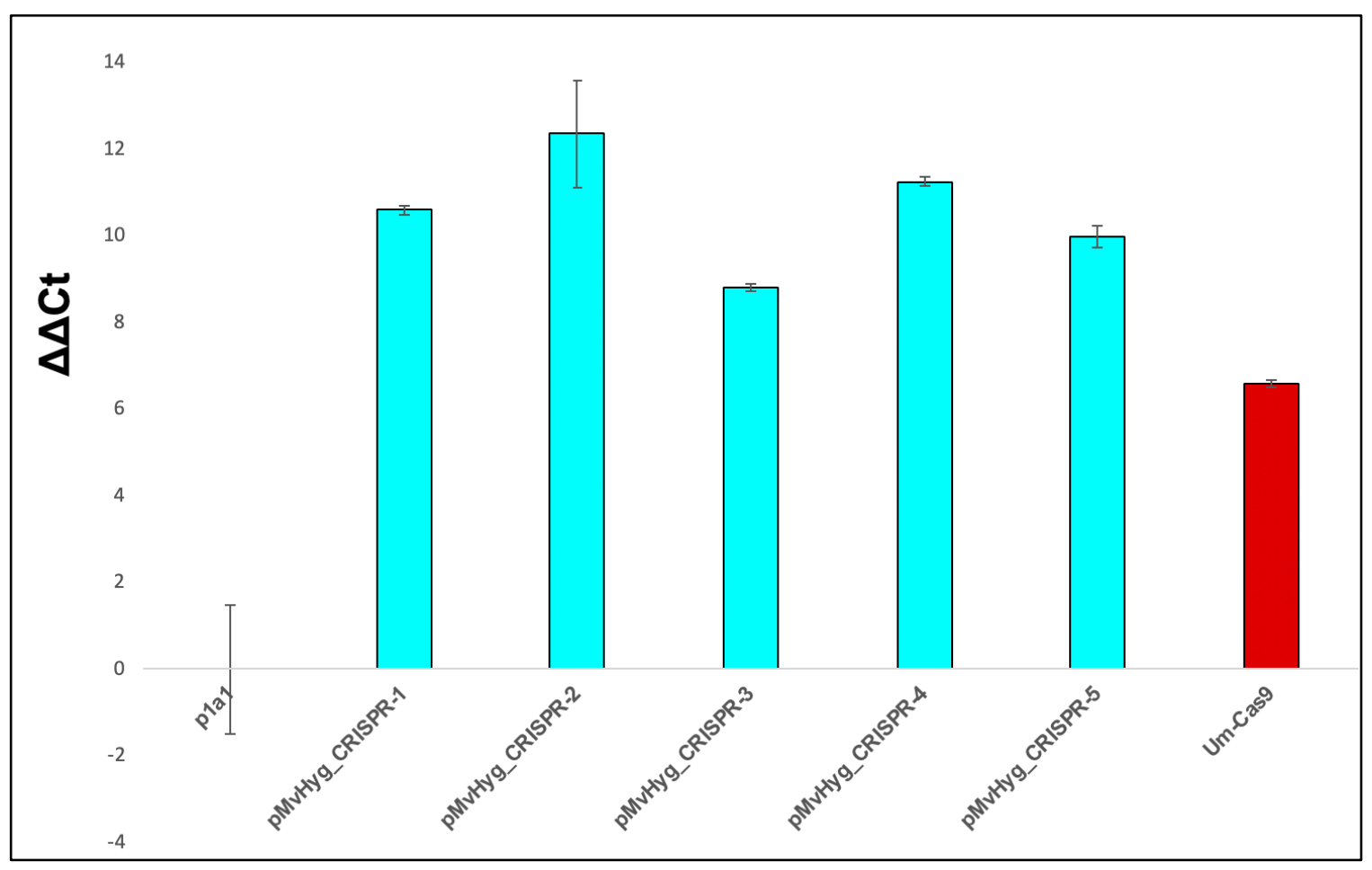

Figure C5-17 qrt PCR expression of Cas9 in Microbotryum. Gene expression was standardized against $\beta$ tubulin expression in wild type p1A1 cells, first lane. Lanes 2-6 represent different transformants collected from selection media. Lane 7 represents a positive control for Cas9 expression using Ustilago maydis transformed with pMS10.

\subsection{PEG Transformation of M. lychnidis-dioicae with Cas9 duplex}

When protoplasted cells were treated with PEG, the Cas9 duplexed with the crRNA/tracrRNA components and repair template, colonies were able to successfully recover on YPD- $10 \%$ media containing Hygromycin $\mathrm{B}$, indicating successful insertion of the repair template into these cells. Furthermore, PCR amplification of the target region demonstrated appropriate size reduction that would be expected to occur with the insertion 
of the 1,801 bp Hygromycin B resistance cassette in-between the two Cas9 cut sites, while excising the 2,212 bp MVLG_05585 gene (Figure C5-18).

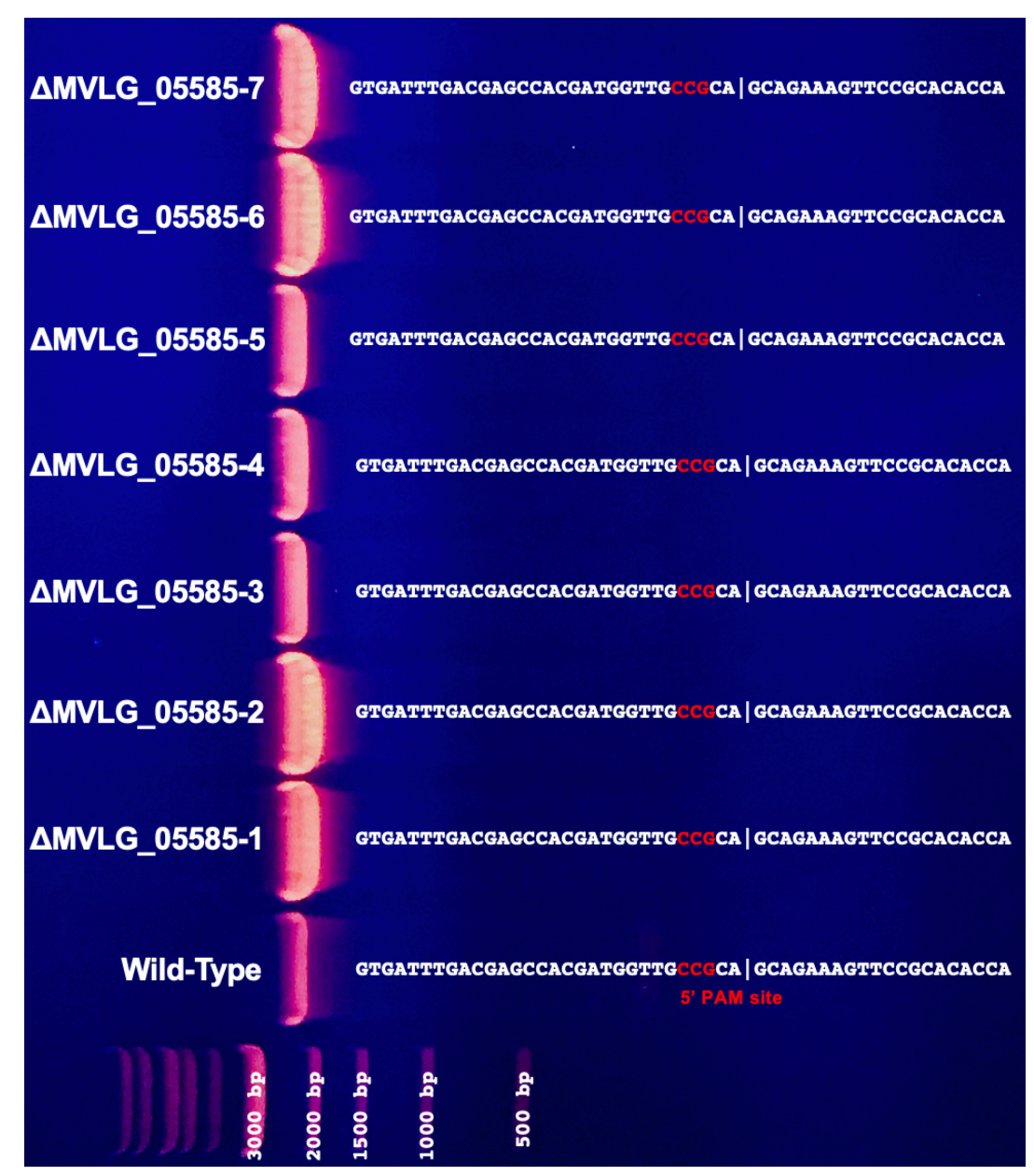

Figure C5-18 PCR verification of potential Cas9 transformants. PCR was performed using primers that amplify the MVLG_05585 coding region of M. lychnidis-dioicae starting 50 base pairs upstream and downstream of the target sites, resulting in a wild-type band of approximately 2,300 bp. Successful disruptions with homologous directed repair should appear as approximately 1,900 bp in length. $1 \mathrm{~kb}$ size standard, purchased from NEB, is shown and labeled on the bottom for size comparison, wild-type cells are shown in lane one, while putative transformants are shown in lanes 2-8. Sequencing results are shown to the right of each strain with the Cas9 PAM site of the target is shown in red. 
However, despite growth on resistance media and seemingly successful PCR verification of MVLG_05585 disruption, follow-up sequencing demonstrates that the 5' and 3' target regions for the MVLG-05585 gene are still intact. This indicates that the Hygromycin B resistance cassette is being inserted into the Microbotryum genome but not in the Cas9 target region.

\section{Discussion}

Implementation of CRISPR-Cas9 in Microbotryum would provide a reliable means for transformation and generating target specific gene disruptions. Doing so would open the door for future analyses of the many novel genes identified by the rapidly expanding library of genomes available for this species complex, genes that are predicted to play a variety of roles in the fungal life cycle and pathogenicity. Additionally, the unique restriction sites on either side of the Cas9 coding gene in both the pMvCC9 and pMvHyg_CRISPR plasmids allow for the use of this systems with other variants of the Cas 9 endonuclease, broadening the scope of application. For example, Cas9 nickase mutants can be used in conjunction with two gRNAs to excise regions of the genome with appropriate overhangs for insertions, and dCas9 double mutants can be used to downregulate expression of a gene by interfering with transcription, a process coined as CRISPRi. Furthermore, as new modifications to the Cas 9 that increase the fidelity of the nuclease activity to reduce off-target cuts being are discovered, these new Cas9 mutants can be easily swapped into pMvCC9-ATMT vector for immediate use in this system.

While we were able to demonstrate the transformation potential of electroporation to introduce material into Microbotryum cells, the inability of cells to grow on selective media indicate either a problem with getting material into the nucleus or issues with maintenance of the pMvCC9 plasmid. One issue may be that the autonomous replication sequence (ARS) used in pMvCC9, which is the ARS used for Ustilago, may not be recognized by Microbotryum, and therefore successful transformants may be unable to propagate the plasmid in their progeny. One approach to addressing this potential problem would be to identify an endogenous ARS from Microbotryum and swapping it in place of the Ustilago ARS in pMvCC9. Currently, there are no known ARS sequences for the system; however, one might generate a library of M. lychnidisdioicae genomic fragments using the Hygromycin resistance cassette as selection to randomly clone in digested fragments of genomic DNA from Microbotryum cells and generate a plasmid containing an ARS. 
These plasmids from the library could then be electroporated into Microbotryum to select for regions of the genomic sequence that contain an endogenous ARS to use for the pMvCC9 plasmid.

Similarly, although we were able to successfully insert the tRNA/gRNA chimera and Cas9 coding regions from pMvHyg_CRISPR into the Microbotryum genome using ATMT, we were not able to observe changes to the MVLG_05585 target region. We were however able to detect expression of the Cas9 enodnuclease via qrtPCR (Figure C5-17). Expression of the Cas9 without proper target editing suggests that the tRNA/gRNA chimera is not properly separating during post transcriptional modification. If the tRNA and gRNA components are not cleaved properly, the tRNA would likely interfere with the binding of Cas9 to the target site and initiation of cutting, even if bound properly to the Cas9 endonuclease. While the current pMvHyg_CRISPR plasmid is not sufficient for targeted gene knockouts, establishment of a Cas9-producing strain of Microbotryum is a step forward and open the door for other transformation possibilities. One alternative would be to remove the tRNA component of the plasmid and replace the U6 promoter that has been demonstrated to drive sgRNA production in Ustilago maydis. Another approach may be to use a duel plasmid system, where the Cas9-producing Microbotryum strain is transformed with another either another vector containing the components for production of a sgRNA or simply the sgRNA or crRNA/tracrRNA itself.

Finally, while attempts to assemble the Cas9 duplex with crRNA/tracrRNA components in vitro before transformation into Microbotryum cells via PEG transformation were the closest thus far at achieving target specific knockouts in M. lychnidis-dioicae, discrepancies between PCR verification and sequencing cast doubts on whether or not modifications are being made appropriately via Cas9 excision of the target gene. Further work using this system should examine the potential for both modified cells and non-modified Microbotryum cells to exist in the same colony. This could be done through t-streaking colonies to assure that they represent a homogenous population of cells, allowing for a more robust identification of potential cells with the desired disruptions.

Given the breadth of its applications, CRISPR represents the addition of a multi-tool to the Microbotryum toolbox. The versatility of pMvCC9 provides the framework for future applications in Microbotryum, including site specific insertions, translational interference and DNA tagging, gene insertions, whole gene excision, and multiple gene knockouts (Hsu et al., 2014). This system will undoubtedly play a vital part in 
future studies seeking to identify the role of many unique genes found amongst closely related species of the Microbotryum genus, shedding light on the molecular mechanisms behind speciation events resulting from co-evolution between the plant hosts and these fungal pathogens. 


\section{CHAPTER 6}

\section{CONCLUSIONS}

The work presented in this dissertation was the first to identify and compare secretomes of various Microbotryum species at the molecular level, to understand the coevolutionary changes that have led to hostspecificity within the Microbotryum genus. This work sets the groundwork for future research into the secreted proteins of the Microbotryum genus by identifying the secretomes portfolios of M. lychnidis-dioicae, M. silenes-dioicae, and M. violaceum var. paradoxa. These lists of secreted proteins act as a starting point for future molecular genetics studies to understand their functions in the hosts. This dissertation also provides insight into the importance of different sets of secreted proteins, including those that are heavily conserved across the Microbotryum genus and those that are unique to each species.

While species-specific secreted proteins can play a role in overall pathogenicity, the more likely cause for adaptation and coevolution over time are small amino acid sequence changes to conserved Microbotryum effectors. Speciation events are therefore likely the result of diversifying selection in different populations of plants/host in which rapid changes to the proteins secreted to block plant defense responses are driven by subsequent rapid changes in mechanisms that detect the presence of the pathogen in the host. The secretomes utilized by different Microbotryum species are largely shared with few gene gains or losses. Instead, small stepwise changes in the amino acid sequences of core secreted proteins likely play a much larger role in host specificity. This is supported by the rapid evolution observed in the core SPs under positive selection compared to the non-secreted proteins observed in the genomes, and the comparatively small number of species-specific genes across the Microbotryum genus. In addition to changes that might alter the function of a secreted protein, this work also identified a mechanism for mobilization or de-mobilization of effectors by changes to the leader sequence of proteins, which could quickly change the arsenal of secreted proteins utilized by each species.

Molecular analysis of one such core secreted protein, MVLG_02245, provides further evidence to support that validity of the predictive measures used in our secretomes analyses. Through Yeast-Secretion- 
Trap methods and Yeast-Two-Hybrid screening, we both demonstrated that the core protein was secreted and likely an effector by demonstrating that the leader sequence codes for secretion of other genes in a yeast system and targets a tubulin $\alpha-1$ chain protein within the Silene latifolia hosts. While further analyses will be needed to visualize the localization of this secreted protein in vivo, interaction with the tubulin $\alpha-1$ chain ortholog in S. latifolia demonstrates a mechanism in which the secreted protein can weaken the hosts in order for the fungal cells to penetrate the cell wall during pathogenesis. Thus, core secreted proteins are likely highly preserved due to their mechanistic role of infection in the host, i.e., contributing to the physical entrance and manipulation of the host.

Species-specific proteins on the other hand seem play a role in regulation and depression of specific host recognition factors and defense response. This research demonstrates that while not all species-specific genes confer an infectious advantage in the Microbotryum species that express them, the MvS1_01693 did significantly improve the pathogenicity of M. silenes-dioicae on its natural host, S. dioica. We hypothesize that the MvSd_01693 transgenic strain of M. silenes-dioicae may be more successful at infecting S. dioica due to the lack of exposure, and subsequent selective adaptation, to the MvSl_01693 effector. An increase in pathogenicity of its own host when expressing the species-specific gene of its sister species indicates that these species-specific genes may be artifacts of co-evolution post speciation events and may be useful for evaluating the evolutionary history and relatedness of members in the Microbotryum genus.

Finally, in effort to further lay the groundwork for future research analyzing the hundreds of secreted proteins identified by this research, this dissertation made progress in establishing a gene-specific knockout system using CRISPR Cas9. While we were unable to make changes to the target region used in our pilot study, this dissertation did make progress towards a reliable site-specific gene modification system by generating a strain of Microbotryum that expresses Cas9. This strain can be combined with a variety of other transformation practices to introduce guide RNA templates to chaperone the Cas9 to a desired target. Furthermore, slight modifications to the amino acid sequence of the plasmid vector used to introduce Cas9 to M. lychnidis-dioicae can be made to broaden the molecular genetic toolbox available for the Microbotryum genus by altering the function of Cas9 to act as a Nickase or for use in CRISPRi methods for gene knockdown studies. 


\section{REFERENCES}

Abascal F, Zardoya R, Telford M. (2010). TranslatorX: multiple alignment of nucleotide sequences guided by amino acid translations. Nucleic Acids Res, 38: 7-13. https://doi.org/10.1093/nar/gkq291

Abrash E, Anleu Gil MX, Matos JL, Bergmann DC. (2018). Conservation and divergence of YODA MAPKKK function in regulation of grass epidermal patterning. Development 145(14). https://doi.org/10.1242/dev.165860

Akhmetov A, Laurent JM, Gollihar j, Gardner EC, Garge RK, Ellington AD, Kachroo AH, Marcotte EM. (2018). Single-step precision genome editing in yeast using CRISPR-Cas9. Bio Protoc 8(6): e2765. https://doi.org/10.21769/BioProtoc.2765

Albain J, Durand EM, Yang S, Zhou Y, Zon LI. (2015). A CRISPR/Cas9 vector system for tissuespecific gene disruption in zebrafish. Dev Cell 32(6): 756-64. https://doi.org/10.1016/j.devcel.2015.01.032

Albersheim R, Anderson AJ. (1971). Proteins from plant cell walls inhibit polygalacturonases secreted by plant pathogens. P Natl Acad Sci USA. 68(8): 1815-1819 https://doi.org/10.1073/pnas.68.8.1815

Altenbuchner J. (2016). Editing of the Bacillus subtilis Genome by CRISPR-Cas9. Appl Environ Microb https://doi.org/10.1128/AEM.01453-16

Anderson JP, et al. (2010). Plants versus pathogens: an evolutionary arms race. Funct Plant Biol. 37(6): 499-512 https://doi.org/10.1071/FP09304

Andersson DI, Jerlström-Hultqvist J, Näsvall J. (2015). Evolution of new functions de novo and from preexisting genes. Cold Spring Harbor Perspectives in Biology. 7(6): a0-17996 https://doi.org/10.1101/cshperspect.1017996

Aguileta G, et al. (2010). Finding candidate genes under positive selection in non-model species: examples of genes involved in host specialization in pathogens. Mol Ecol.

https://doi.org/10.1111/j.1365-294X.2009.04454.x 
Auld SKJR, Tinkler SK, Tinsley MC. (2016). Sex as a strategy against rapidly evolving parasites. Proc. $R$. Soc. B 283: 20162226. http://dx.doi.org/10.1098/rspb.2016.2226

Badouin $\mathrm{H}$, et al. (2017). Widespread selective sweeps throughout the genome of model plant pathogenic fungi and identification of effector candidates. Mol Ecol. 26(7): 2041-2062 doi: $10.1111 / \mathrm{mec} .13976$.

Baker HG. (1947). Infection of species of Melandrium by Ustilago violacea (Pers.) Fuckel and the transmission of the resultant disease. Ann. Bot. (Lond.), 11: 333-348

Bebber PB, Gurr SJ. (2015). Crop-destroying fungal and oomycete pathogens challenge food security. Fungal Genet Biol. 74: 62-64 doi: 10.1016/j.fgb.2014.10.012

Beckerson WC, de la Vega RCR, Hartmann FE, Duhamel M, Giraud T, Perlin MH. (2019). Cause and Effectors: Whole genome comparisons reveal shared but rapidly evolving effector sets among host-specific plant-castrating fungi. mBio. mBio 10:e2391-19 https://doi.org/10.1128/mBio.02391-19

Begerow D, Nilsson H, Unterseher M, Maier W. (2010). Current state and perspectives of fungal DNA barcoding and rapid identification procedures. Appl Microbiol biot. 87(1): 99-108 doi: $10.1007 / \mathrm{s} 00253-010-2585-4$

Bergstrom CT, Dugatkin LA. (2011). Evolution. NY \& London: W. W. Norton \& Company.

Bier E, Harrison MM, O'Connor-Giles KM, Wildonger J. (2018). Advances in engineering the fly genome with the CRISPR-Cas system. Genetics 208(1): 1-18. https://doi.org/10.1534/genetics.117.1113

Bierle CH, Anderholm KM, Wang JB, McVoy MA, Schleiss MR. (2016). Targeted mutagenesis of guinea pig cytomegalovirus using CRISPR/Cas9-mediated gene editing. J Virol 90(15): 69896998. https://doi.org/10.1128/JVI.oo139-16

Blümke A, Falter C, Herrfurth C, Sode B, Bode R, Schäfer W, Feussner I, Voigt CA. (2014). Secreted fungal effector lipase releases free fatty acids to inhibit innate immunity-related callose formation during wheat head infection. Plant Physiology. 165(1): 346-358 https://doi.org/10.1104/pp.114.236737

Brameier M, Krings A, MacCallum RM. (2007). NucPred - predicting nuclear localization of proteins. Bioinformatics. 23(9): 1159-60 DOI: 10.1093/bioinformatics/btm066 
Branco S, et al. (2018). Multiple convergent supergene evolution events in mating-type chromosomes.

Nature Communications. 9: 2000 doi: 10.1038/s41467-018-04380-9

Brown NA, Hammond-Kosack KE. Secreted biomolecules in fungal plant pathogenesis. In: Gupta VK, Mach RL, Sreenivasaprasad S, editors. Fungal Biomolecules: Sources, Applications and Recent Developments. Oxford, UK: John Wiley \& Sons, Ltd; 2015. pp. 263-310.

Casa-Esperón Edl. (2012). Horizontal transfer and the evolution of host-pathogen interactions. International Journal of Evolutionary Biology. 2012: 1-9 https://doi.org/10.1155/2012/679045

Char SN, Neelakandan AK, Nahampun H, Frame B, Main M, Spalding MH, Becraft PW, Meyers BC, Walbot V, Wang K, Yang B. (2017). An Agrobacterium-Delivered CRISPR/Cas9 System for High-Frequency Targeted Mutagenesis in Maize. Plant Biotechnol J 15(2): 257-268 https://doi.org/10.1111/pbi.12611

Chen B, Gilbert LA, Cimini BA, Schnitzbauer J, Zhang W, Li GW, Park J, Blackburn EH, Weissman JS, Qi LS, Huang B. (2013). Dynamic Imaging of Genomic Loci in Living Human Cells by an Optimized CRISPR/Cas System. Cell 155(7): 1479-91 https://doi.org/10.1016/j.cell.2013.12.001

Chen B-X, Wei T, Zhi-Wei Y, Yun F, Kang L-Z, Tang H-B, Guo L-Q, Lin J-F. (2018). Efficient CRISPR -Cas9 gene disruption system in edible-medicinal mushroom Cordyceps militaris. Front Microbiol 9: 1157. https://doi.org/10.3389/fmicb.2018.01157

Chen W, Zhang Y, Zhang Y, Pi Y, Gu T, Song L, Wang Y, Ji Q. (2018). CRISPR/Cas9-based genome editing in Pseudomonas aeruginosa and cytidine deaminase-mediated base editing in Pseudomonas species. iScience 6: 222-231 https://doi.org/10.1016/j.isci.2018.07.024

Chew WL, Tabebordbar M, Cheng JKW, Mali P, Wu EY, Ng AHM, Zhu K, Wagers AJ, and Church GM. (2016). A Multifunctional AAV-CRISPR-Cas9 and Its Host Response. Nat Met 13(10): 868-74 https://doi.org/10.1038/nmeth.3993

Cong L, Ran FA, Cox D, Lin S, Barretto R, Habib N, Hsu PD, Wu X, Jiang W, Marraffini LA, Zhang F. (2013). Multiplex Genome Engineering Using CRISPR/Cas Systems. Science 339(6121): 819-23 https://doi.org/10.1126/science. 1231143

Danecek P, et al. (2011). The variant call format and VCFTOOLS. Bioinformatics, 27: 2156-2158. https://doi.org/10.1093/bioinformatics/btr330 
Davies WJ, Kudoyarova G, Hartung W. (2005). Long-distance ABA signaling and its relation to other signaling pathways in the detection of soil drying and the mediation of the plant's response to drought. Journal of Plant Growth and Reguationl, 24: 285-295

DOI: $10.1007 / \mathrm{s} 00344-005-0103-1$

de Visser JAGM, Elena SF. (2007). The evolution of sex: empirical insights into the roles of epistasis and drift. Nature Reviews: Genetics. 8: 139-149 https://doi.org/10.1038/nrg1985

Dean R, Van Kan JA, Pretorius ZA, Hammond-Kosack KE, Di Pietro A, Spanu PD, Rudd JJ, Dickman M, Kahmann R, Ellis J, Foster GD. (2012). The top 10 fungal pathogens in molecular plant pathology. Mol Plant Pathol. 13(4): 414-430 doi: 10.1111/j.1364-3703.2011. 00783.x

Delaunois B, Jeandet P, Clément C, Baillieul F, Dory S, Cordelier S. (2014). Uncovering plant-pathogen crosstalk through apoplastic proteomic studies. Frontiers in Plant Science. 5(249): 1-18 https://doi.org/10.3389/fpls.2014.00249

Demirci Y, Zhang B, Unver T. (2017). CRISPR/Cas9: An RNA-guided highly precise synthetic tool for plant genome editing. J Cell Physiol 233(3) https://doi.org/10.1002/jcp.25970

Dickinson DJ, Goldstein B. (2016). CRISPR-Based Methods for Caenorhabditis elegans genome engineering. Genetics 202(3) https://doi.org/10.1534/genetics.115.182162

Dita M, Barquero M, Heck D, Mizubuti ESG, Staver CP. (2018). Fusarium Wilt of Banana: Current Knowledge on Epidemiology and Research Needs Toward Sustainable Disease Management. Front. Plant Sci. https://doi.org/10.3389/fpls.2018.01468

Doll NM, Gilles LM, Gérentes MF, Richard C, Just J, Fierlej Y, Borrelli VMG, Gendrot G, Ingram FC, Rogowsky PM, Widiez T. (2019). Single and multiple gene knockouts by CRISPRCas9 in maize. Plant Cell Rep 38(4):487-501 https://doi.org/10.1007/s00299-019-02378-1

Dongen S, Graph clustering by flow simulation. PhD thesis, University of Utrecht, May 2000. Available at https://micans.org/mcl/

Doron-Faigenboim A, Stern A, Mayrose I, Bacharach E, Pupko T. (2005). Selecton: a server for detecting evolutionary forces at a single amino-acid site. Bioinformatics. 21: 2101-2103

DOI: 10.1093/bioinformatics/bti259

Droit A, Poirier GG, Hunter JM. (2005). Experimental and bioinformatic approaches for interrogating 
protein-protein interactions to determine protein function. Journal of Molecular Endocrinology. 34(2) https://doi.org/101677/jme.1.01693

Duplessis S, Cuomo CA, Lin Y-C, Aerts A, Tisserant E, Veneault-Fourrey C, Joly DL, Hacquard S, Amselem J, Cantarel BL, Chiu R, Coutinho PM, Feau N, Field M, Frey P, Gelhaye E, Goldberg J, Grabherr MG, Kodira CD, Kohler A, Kües U, Lindquist EA, Lucas SM, Mago R, Mauceli E, Morin E, Murat C, Pangilinan JL, Park R, Pearson M, Quesneville H, Rouhier N, Sakthikumar S, Salamov AA, Schmutz J, Selles B, Shapiro H, Tanguay P, Tuskan GA, Henrissat B, Van de Peer Y, Rouzé P, Ellis JG, Dodds PN, Schein JE, Zhong S, Hamelin RC, Grigoriev IV, Szabo LJ, Martin F. (2011). Obligate biotrophy features unraveled by the genomic analysis of rust fungi. Proc Natl Acad Sci. 108: 9166-71 doi: 10.1073/pnas.1019315108

Dwivedi S, Goldman I, Ortiz R. 2019. Pursuing the potential of heirloom cultivars to improve adaptation, nutritional, and culinary features of food crops. MDPI Agronomy. 9(8): 441 https://doi.org/10.3390/agronomy9080441

Eisenhaber F. (2013). Prediction of Protein Function Two Basic Concepts and One Practical Recipe. Austin TX: Landes Bioscience; 2000-2013

Edgar R. (2004). MUSCLE: multiple sequence alignment with high accuracy and high throughput. Nucleic Acids Res. 32 1792-1797 DOI: 10.1093/nar/gkh340

Ekseth OK, Kuiper M, Mironov V. (2014). orthAgogue: an agile tool for the rapid prediction of orthology relations. Bioinformatics. 30(5): 734-735 doi: 10.1093/bioinformatics/btt582

El-Gebali S, Mistry J, Bateman A, Eddy SR, Luciani A, Potter SC, Qureshi M, Richardson LJ, Salazar GA, Smart A, Sonnhammer ELL, Hirsh L, Paladin L, Piovesan D, Tosatto SCE, Finn RD. (2019). The Pfam protein families database in 2019. Nucleic Acids Res. 47(D1): D427-D432 doi: $10.1093 /$ nar/gky995

Elmore JR, Yokooji Y, Sato T, Olson S, Glover III CVC, Graveley BR, Atomi H, Terns RM, Terns MP. (2013). Programmable plasmid interference by the CRISPR-Cas system in Thermococcus kodakarensis. RNA Biol 10(5): 828-840 https://doi.org/10.4161/rna.24084

Emanuelsson O, Nielsen H, Brunak S, von Heijne G. (2000). Predicting subcellular localization of proteins based on their N-terminal amino acid sequence. J Mol Biol. 300(4): 1005-1016 
DOI: $10.1006 /$ jmbi.2000.3903

Erbs G, Newman MA. (2012). The role of lipopolysaccharide and peptidoglycan, two glycosylated bacterial microbe-associated molecular patterns (MAMPs), in plant innate immunity. Mol. Plant Pathol. 13: 95-104 https://doi.org/10.1111/j.1364-3703.2011.00730.x

Erickson PA, Ellis NA, Miller CT. (2016). Microinjection for transgenesis and genome editing in Threespine Sticklebacks. JOVE-J Vis Exp (111) https://doi.org/10.3791/54055

Eun K, Park MG, Jeong YW, Jeong YI, Hyun S-H, Hwang WS, Kim S-H, Kim H. (2019). Establishment of TP53-knockout canine cells using optimized CRISPR/Cas9 vector system for canine cancer research. BMC Biotech 19: 1 https://doi.org/10.1186/s12896-018-0491-5

Fan D, Liu R, Li C, Jiao B, Li S, Hou Y, Luo K. (2015). Efficient CRISPR/Cas9-mediated targeted mutagenesis in Populus in the first generation. Sci Rep-UK 5:12217 https://doi.org/10.1038/srep12217

Fan Y, Lin X. (2018). Multiple application of transient CRISPR-Cas9 coupled with electroporation (TRACE) system in the Cryptococcus neoformans species complex. Genetics 208(4): 1357-1372. doi: $10.1534 /$ genetics.117.300656

Fan Z, Li W, Lee SR, Meng Q, Shit B, Bunch TD, White KL, Kong I-K, Wang Z. (2014). Efficient gene targeting in golden Syrian hamsters by the CRISPR/Cas9 system. PLOS ONE. 9(10): e109755 https://doi.org/10.1371/journal.pone.0109755

Fei J-F, Lou W P-K, Knapp D, Murawala P, Gerber T, Taniguchi Y, Nowoshilow S, Khattak S, Tanaka EM. (2018). Application and optimization of CRISPR-Cas9-mediated genome engineering in axolotl (Ambystoma mexicanum). Nat Protoc 13:2908-2943. https://doi.org/10.1038/s41596-018-0071-0

Fischer GW and Holton CS. (1957). Biology and control of the smut fungi. New York, N.Y: The Ronald Press Company

Fisher MC, Henk DA, Briggs CJ, Brownstein JS, Madoff LC, McCraw SL, Gurr SJ. (2012). Emerging fungal threats to animal, plants, and ecosystem health. Nature. 484(7393): 186 https://doi.org/10.1038/nature10947

Fiza S, Ahmad S, Noor MA, Wang X, Younas A, Riaz A, Riaz A, Ali F. (2019). Applications of the 
CRISPR/Cas9 system for rice grain quality improvement: perspectives and opportunities. Int $J$ Mol Sci 20(4) https://doi.org/10.3390/ijms20040888

Foster AJ, Martin-Urdiroz MM, Yan X, Wright S, Soanes DM, Talbot NJ. (2018). CRISPR-Cas9 ribonucleoprotein-mediated co-editing and counter selection in rice blast fungus. BioRxiv. https://doi.org/10.1101/349134

Froger A, and Hall JE. (2007). Transformation of Plasmid DNA into E. Coli Using the Heat Shock Method. JOVE-J Vis Exp (6):253 https://doi.org/10.3791/253

Fudal I, Ross S, Brun H, Besnard AL, Ermel M, Kuhn ML, Balesdent MH, Rouxel T. (2009). Repeatinduced point mutation (RIP) as an alternative mechanism of evolution toward virulence in Leptosphaeria maculans. Mol Plant Microbe Interact. 22(8): 932-41. https://doi.org/10.1094/MPMI-22-8-0932

Gacura MD, Sprockett DD, Heidenreich B, Blackwood CB. (2016). Comparison of pectin-degrading fungal communities in temperate forests using glycosyl hydrolase family 28 pectinase primers targeting Ascomycete fungi. J Microbiol Methods. Apr;123:108-13.

https://doi.org/10.1016/j.mimet.2016.02.013

Garber ED and Day AW. (1985). Genetic mapping of a phytopathogenic basidiomycete Ustilago violacea. Bot Gaz. 146(4): 449-459

Gasiunas G, Barrangou R, Horvath P, Siksnys V. (2012). Cas9-crRNA ribonucleoprotein complex mediates specific DNA cleavage for adaptive imunity in bacteria. PNAS 109(39)15539-15540. www.pnas.org/cgi/doi/10.1073/pnas.1208507109

Gaudelli NM, Komor AC, Rees HA, Packer MS, Badran AH, Bryson DI, Liu DR. (2017). Programmable base editing of A-T to G-C in genomic DNA without DNA cleavage. Nature. 551(7681): 464-471 doi: $10.1038 /$ nature24644

Ghareeb H, et al. (2010). Pathogenicity determinants in smut fungi revealed by genome comparison. Science. 330(6010): 1546-1548 doi: 10.1126/science.1195330

Gibson AK, Refrégier G, Hood ME, Giraud T. (2014). Performance of a hybrid fungal pathogen on purespecies and hybrid host plants. Int J Plant Sci. 175(6): 724-730 DOI: 10.1086/676621

Gibson DG, Young L, Chuang RY, Venter JC, Hutchison CA, and Smith HO. (2009). Enzymatic 
Assembly of DNA Molecules up to Several Hundred Kilobases. Nat Methods 6(5):343-5 https://doi.org/10.1038/nmeth.1318

Gratz SJ, Dustin Rubinstein C, Harrison MM, Wildonger J, O'Connor-Giles KM. (2015). CRISPRCas9 genome editing in Drosophila. Curr Protoc Mol Biol https://doi.org/10.1002/0471142727.mb3102s111

Grigg ME, Suzuki Y. (2003). Sexual recombination and clonal virulence in Toxoplasma. Microbes Infect. 5(7): 685-90 https://doi.10.1016/s1286-4579(03)00088-1

Gruzmán-Zapata D, Sandoval-Vargas JM, Macedo-Osorio KS, Salgado-Manjarrez E, Castrejón-Flores JL, Oliver-Salvador MdC, Durán-Figueroa NV, Nogué F, Badillo-Corona JA. (2019). Efficient editing of the nuclear APT reporter gene in Chlamydomonas reinhardtii via expression of a CRISPR-Cas9 module. Int J Mol Sci 20(5):1247 https://doi.org/10.3390/ijms20051247

Hartmann FE, Croll D. (2018). Distinct trajectories of massive recent gene gains and losses in populations of a microbial eukaryotic pathogen. Mol Biol Evol. 34(11): 2808-2822 doi: $10.1093 / \mathrm{molbev} / \mathrm{msx} 208$

Hartmann FE, Rodríguez de la Vega RC, Carpentier F, Gladieux P, Cornille A, Hood ME, Giraud T. (2019). Understanding Adaptation, Coevolution, Host Specialization, and Mating System in Castrating Anther-Smut Fungi by Combining Population and Comparative Genomics. Annu Rev Phytopathol. 57: 431-457 doi: 10.1146/annurev-phyto-082718-095947

Harel I, Valenzano DR, Brunet A. (2016). Efficient genome engineering approaches for the short-lived African turquoise killifish. Nat Protoc 11:2010-2028 https://doi.org/10.1038/nprot.2016.103 Hartmann FE, Rodríguez de la Vega RC, Brandenbrug J-T, Carpentier F, Giraud T. (2018). Gene presenceabsence polymorphism in castrating anther-smut fungi: recent gene gains and phylogeorgraphic structure. Genome Biol Evol. 10(5): 1298-1314 doi: 10.1093/gbe/evy089

Hashimoto T. (2015). Microtubules in Plants. The Arabidopsis Book. 13: e0179 https://doi.org/10.1199/tab.0179

Hasman H, Saputra D, Sicheritz-Ponten T, Lund O, Svendsen CA, Frimodt-Møller N, Aarestrup FM. Rapid whole-genome sequencing for detection and characterization of microoganisms directly from clinical samples. Journal of Clinical Microbiology. 52(1): 139-146 
https://doi.org/10.1128/JCM.02452-13

Heitman J, Carter DA, Dyer PS, Soll DR. (2014). Sexual reproduction of human fungal pathogens. Cold Spring Harbor Perspectives in Medicine. 4(8): a019281

https://doi.org/10.1101/cshperspect.a019281

Hindriksen S, Bramer AJ, Truong MA, Vromans MJM, Post JB, Verlaan-Klink I, Snippert HJ, Lens SMA, Hadders MA. (2017). Baculoviral delivery of CRISPR/Cas9 facilitates efficient genome editing in human cells. PLOS ONE 12(6):e0179514

doi: 10.1371 journal.pone. 0179514

Hirose M, Hasegawa A, Mochida K, Matoba S, Hatanaka Y, Inoue K, Goto T, Kaneda H, Yamada I, Furuse T, Abe K, Uenoyama Y, Tsukamure H, Wakana S, Honda A, Ogura A. (2017).

CRISPR/Cas9-mediated genome editing in wild-derived mice: generation of tamed wild-derived strains by mutation of the a (nonagouti) gene. Sci Rep-UK 7:42476

https://doi.org/10.1038/srep42476

Hiruta C, Kakui D, Tollefsen KE, Iguchi T. (2018). Targeted gene disruption by use of CRISPR/Cas9 ribonucleoprotein complexes in the water flea Daphnia pulex. Genes Cells 23(6): 494-502. https://doi.org/10.1111/gtc. 12589

Hofmann NR. (2017). An emerging paradigm? RxLR cleavage before effector section. Plant Cell. 29: 1177-1178 doi: $10.1105 /$ tpc. 17.00454

Hood ME, Katawczik M, Giraud T. (2005). Repeat-induced point mutation and the population structure of transposable elements in Microbotryum violaceum. Genetics. 170(3): 1081-1089

DOI: 10.1534 /genetics. 105.042564

Hood ME, et al. (2010). Distribution of the anther-smut pathogen Microbotryum on species of the Caryophyllaceae. New Phytol. 187: 217-229 doi: 10.1111/j.1469-8137.2010.03268.x

Hopes A, Nekrasov V, Kamoun S, Mock T. (2016). Editing of the urease gene by CRISPR-Cas in the diatom Thalassiosira pseudonana. Plant methods 12:49. https://doi.org/10.1186/s13007-0160148-0

Horns F, Petit E, Yockteng R, Hood ME. (2012). Patterns of repeat-induced point mutation in transposable 
elements of basidiomycete fungi. Genome Biol Evol. 4(3): 240-7

https://doi.org/10.1093/gbe/evs005

Horvath P, and Barrangou R. (2010). CRISPR/Cas, the Immune System of Bacteria and Archaea. Science 327(5962):167-70 https://doi.org/10.1126/science.1179555

Hu JH, Miller SM, Geurts MH, Tang W, Chen L, Sun N, Zeina CM, Gao X, Rees HA, Lin Z, Liu DR. (2018). Evolved Cas9 variants with broad PAM compatibility and high DNA specificity. Nature 556(7699):57-63 https://doi.org/10.1038/nature26155

Huang Y, Niu B, Gao Y, Fu L, Li W. (2010). CD-HIT Suite: a web server for clustering and comparing biological sequences. Bioinformatics. 26(5): 680-2 doi: 10.1093/bioinformatics/btq003

Hulsen T, de Vlieg J, Alkema W. (2008). BioVenn - a web application for the comparison and visualization of biological lists using area-proportional Venn diagrams. BMC Genomics. 9: 488 doi: $10.1186 / 1471-2164-9-488$

Hwang WY, Fu Y, Reyon D, Maeder ML, Kaini P, Sander JD, Joung JK, Peterson RT, Yeh JR. (2013). Heritable and precise zebrafish genome editing using a CRISPR-Cas system. PLoS ONE 9;8(7) e68708 https://doi.org/10.1371/journal.pone.0068708

Hsu PD, Lander ES, Zhang F. (2014). Development and Applications of CRISPR-Cas9 for Genome Engineering. 157(6):1262-78 Cell https://doi.org/10.1016/j.cell.2014.05.010

Ikmi A, McKinney SA, Delventhal KM, Gibson MC. (2014). TALEN and CRISPR/Cas9-mediated genome editing in the early-branching metazoan Nematostella vectensis. Nat Commun 5: 5486 https://doi.org/10.1038/ncomms6486

Ishino Y, Shinagawa H, Makino K, Amemura M, and Nakatura A. (1987). Nucleotide Sequence of the Iap Gene, Responsible for Alkaline Phosphatase Isoenzyme Conversion in Escherichia Coli, and Identification of the Gene Product. J Bacteriol 169(12): 5492-5433 https://doi.org/10.1128/jb.169.12.5429-5433.1987

Ismagul A, Yang N, Maltseva E, Iskakova G, Mazonka I, Skiba K, Bi H, Eliby S, Jatayev S, Yuri S, Borisjuk N, Langridge P. (2018). A Biolistic Method for High-Throughput Production of Transgenic Wheat Plants with Single Gene Insertions. BMC Plant Biol 18: 135 https://doi.org/10.1186/s12870-018-1326-1 
Jansing J, Sack M, Augustin SM, Fischer R, Bortesi L. (2018). CRISPR/Cas9-mediated knockout of six glycosyltransferase genes in Nicotiana benthamiana for the production of recombinant proteins lacking $\beta$-1,2-xylose and core a-1,3,-fucose. Plant Biotechnol J 17(2) https://doi.org/10.111/pbi.12981

Jansen R, Embden DD, Gaastra W, Schouls LM. (2002). Identification of genes that are associated with DNA repeats in prokaryotes. Mol Microbiol 43(6): 1565-75. https://doi.org/10.1046/j.1365-2958.2002.02839.x

Jian Y, Chen B, Duan C, Sun B, Yang J, Yang S. (2016). Multigene editing in the Escherichia coli genome via the CRISPR-Cas9 system. Appl Environ Microb 81(7): 2506-2514. https://doi.org/10.1128/AEM.04023-14

Jiménez A, Muñoz-Fernández G, Ledesma-Amaro R, Buey RM, Revuelta JL. (2019). One-vector CRISPR/Cas9 genome engineering of the industrial fungus Ashbya gossypii. Microb Biotechnol https://doi.org/10.1111/1751-7915.13425

Jinek M, Chylinski K, Fonfara I, Hauer M, Doudna JA, Charpentier E. (2012). A programmable dualRNA-guided DNA endonuclease in adaptive immunity. Science 337(6096): 816-821. https://doi.org/10.1126/science.1225829

Jones DAB, Bertazzoni S, Turo CJ, Syme RA, Hane JK. (2018). Bioinformatic prediction of plantpathogenicity effector proteins of fungi. Curr Opin Microbiol. 46: 43-49. doi: 10.1016/j.mib.2018.01.017

Jones JDG, Dangl JL. (2006). The plant immune system. Nature. 444: 323-329 https://doi.org/10.1038/nature05286

Juge N. (2006). Plant protein inhibitors of cell wall degrading enzymes. Trends Plant Sci. 2006 Jul;11(7): 359-67 DOI: 10.1016/j.tplants.2006.05.006

Jung T-Y, Park K-H, An Y, Schulga A, Deyev S, Jung J-H, Woo E-J. (2016). Structural features of Cas2 from Thermococcus onnurineus in CRISPR-cas system type IV. Protein Sci 25(10): 1890-1897. https://doi.org/10.1002/pro.2981

Käll L, Krogh A, Sonnhammer EL. (2007). Advantages of combined transmembrane topology and signal peptide predition - the Phobius web server. Nucleic Acids Res. 35: W429-32 
DOI: $10.1093 / \mathrm{nar} / \mathrm{gkm} 256$

Kato-Unoki Y, Takai Y, Kinoshita M, Mochizuki T, Tatsuno T, Shimasaki Y, Oshima Y. (2018).

Genome editing of pufferfish saxitoxin- and tetrodotoxin-binding protein type 2 in Takifugu rubripes. Toxicon. 153:58-61. https://doi.org/10.1016/j.toxicon.2018.08.001

Keyhani NO. (2018). Lipid biology in fungal stress and virulence: Entomopathogenic fungi._Fungal Biol. 122(6): 420-429 https://doi.org/10.1016/j.funbio.2017.07.003

Khedr MA, Massarotti A, Mohamed ME. (2018). Rational discovery of (+) (S) abscisic acid as a potential antifungal agent: a repurposing approach. Sci. Rep. 8(1): 8565 https://doi.org/10.1038/s41598-018-26998-X

Kim S, Bae T, Hwang J, and Kim JS. (2017). Rescue of High-Specificity Cas9 Variants Using SgRNAs with Matched 5' Nucleotides. Genome Biol. 18:218 https://doi.org/10.1186/s13059-017-1355-3

Kleinstiver BP, Pattanayak V, Prew MS, Tsai SQ, Nguyen NT, Zheng Z, and Joung JK. (2016). High-Fidelity CRISPR-Cas9 Nucleases with No Detectable Genome-Wide off-Target Effects. Nature 529(7587): 468-9 https://doi.org/10.1038/nature16526

Klarzynski O, Plesse B, Joubert J-M, Yvin J-C, Kopp M, Kloareg B, Fritig B. (2000). Linear $\beta-1,3$ glucans are elicitors of defense responses in tobacco. Plant Physiology. https://doi.org/10.1104/pp.124.3.1027

Klompe SE, Vo PLH, Halpin-Healy TS, Sternberg SH. (2019). Transposon-encoded CRISPR-Cas systems direct RNA-guided DNA integration. Nature. https://doi.org/10.1038/s41586-019-1323-z

Kobayashi T, Namba M, Koyano T, Fukushima M, Sato M, Ohtsuka M, Matsuyama M. (2018). Successful production of genome-edited rats by the rGONAD method. BMC Biotech 18:19. https://doi.org/10.1186/s12896-018-0430-5

Komor AC, Kim YB, Packer MS, Zuris JA, Liu DR. (2016). Programmable editing of a target base in genomic DNA without double-stranded DNA Cleavage. 533: 420-424 https://doi.org/10.1038/nature17946

Korber B. (2000). HIV signature and sequence variation analysis. Computational analysis of HIV molecular sequences, chapter 4, pages 55-72. Allen G. Rodrigo and Gerald H. Learns, eds. Dordrecht, Netherlands: Kluwer Academic Publishers 
Kost TA, Condreay JP, and Jarvis DL. (2005). Baculovirus as Versatile Vectors for Protein Expression in Insect and Mammalian Cells. Nat Biotechnol 23(5): 567-75 https://doi.org/10.1038/nbt1095

Krogh A, Larsson B, von Heijne G, Sonnhammer EL. (2001). Predicting transmembrane protein topology with hidden Markov model: application to complete genomes. J Mol Biol. 305(3): 567-580 DOI: $10.1006 /$ jmbi.2000.4315

Kunik T, Tzfira T, Kapulnik Y, Gafni Y, Dingwall C, and Citovsky V. (2001). Genetic Transformation of HeLa Cells by Agrobacterium. P Natl A of Sci 98(4): 1871-6 https://doi.org/10.1073/pnas.98.4.1871

Kuppireddy VS, Uversky VN, Toh SS, Tsai MC, Beckerson WC, Cahill C, Carman B, Perlin MH. (2017). Identification and initial characterization of effectors of an anther smut fungus and the potential host target proteins. Int J Mol Sci, 18: 2489 doi: 10.3390/ijms18112489

Lander E. (2016). The heroes of CRISPR. Cell. https://doi.org/10.1016/j.cell.2015.12.041

Langmead B, Trapnell C, PopM, Salzberg SL. (2009). Ultrafast and memory efficient alignment of short DNA sequences to the human genome. Genome Biol. 10(3):R25 https://doi.org/10.1186/gb-2009-10-3-r25

Lanver D, Tollot M, Schweizer G, Lo Presti L, Reissmann S, Ma LS, Schuster M, Tanaka S, Liang L, Ludwig N, Kahmann R. (2017). Ustilago maydis effectors and their impact on virulence. Nat Rev Microbiol. 15: 409-421 doi: 10.1038/nrmicro.2017.33

Le Gac M, Hood ME, Giraud T. (2007). Evolution of reproductive isolation within a parasitic fungal species complex. Evolution. 61-7: 1781-1787 DOI: 10.1111/j.1558-5646.2007.00144.x

Lee AH, Hurley B, Felsensteiner C, Yea C, Wenzislava C, Bartetzko V, Wang PW, Quach V, Lewis JD, Liu YC, Börnke F, Angers S, Wilde A, Guttman D, Desveaux D. 2012. A bacterial acetyltransferase destroys plant microtubule networks and blocks secretion. PLOS Pathogens. 8(2): e1002523 https://doi.org/10.1371/journal.ppat.1002523

Lee SJ and Rose JK. (2012). A yeast secretion trap assay for identification of secreted proteins from eukaryotic phytopathogens and their plant hosts. Methods Mol Biol, 835: 519-30 doi: 10.1007/978-1-61779-501-5_32

Li M, Liu H, Han J, Liu J, Wang R, Zhao D, Zhou J, Xiang H. (2013). Characterization of CRISPR 
RNA biogenesis and Cas6 cleavage-mediated inhibition of a provirus in the haloarchaeon Haloferax mediterranei. J Bacteriol 195(4): 867-75 https://doi.org/10.1128/JB.01688-12

Li M, Zhao L, Page-McCaw PS, Chen W. (2016). Zebrafish genome engineering using the CRISPR-Cas9 system. Trends Genet 32(12):815-827 https://doi.org/10.1016/j.tig.2016.10.005

Li Y, Pan S, Zhang Y, Ren M, Feng M, Peng N, Chen L, Liang YX, She Q. (2016). Harnessing Type I and Type III CRISPR-Cas systems for genome editing. Nucleic Acids Res 44(4). https://doi.org/10.1093/nar/gkv1044

Liu J, Wang Y, Lu Y, Zheng P, Sun J, Ma Y. (2017). Development of a CRISPR/Cas9 genome editing toolbox for Corynebacterium glutamicum. Microbial Cell Factories 16:205 https://doi.org/10.1186/s12934-017-0815-5

Liu Q, Yuan Y, Zhu F, Hong Y, Ge R. (2018). Efficient genome editing using CRISPR/Cas9 ribonucleoprotein approach in cultured Medaka fish cells. Biol. Open. 7(8) https://doi.org/10.1242/bio.035170

Liu Y, Wang Y, Xu S, Tang X, Zhao J, Yu C, He G, Xu H, Wang S, Tang Y, Fu C, Ma Y, Zhou G. (2019). Efficient genetic transformation of CRISPR/Cas9-mediated genome editing in Lemna aequinoctialis. Plant Biotechnol J. 1-10 https://doi.org/10.1111/pbi.13128

Liu Z. (2017). Inverse gene-for-gene interactions contribute additively to tan spot susceptibility in wheat. Theor Appl Genet. 130(6): 1267-1276 https://doi.org/10.1007/s00122-017-2886-4

Liu Z and Friesen TL. (2012). Polyethylene Glycol (PEG)-Mediated Transformation in Filamentous Fungal Pathogens. Met Mol Biol 835:365-75 https://doi.org/10.1007/978-1-61779-501-5_21

Lommel M, Tursch A, Rustarazo-Calvo L, Trageser B, Holstein TW. (2017). Genetic knockdown and knockout approaches in Hydra. Semantic Scholar. https://doi.org/10.1101/230300

Lopez-Obando M, Hoffmann B, Géry C, Guyon-Debast A, Téoulé E, Rameau C, Bonhomme S, Nogué, F. (2016). Simple and efficient targeting of multiple genes through CRISPR-Cas9 in Physcomitrella patens. G3 (Bethesda) 6(11):3647-3653. https://doi.org/10.1534/g3.116.033266 Louis Carroll. (1871). Through the Looking-Glass and What Alice Found There. Chicago, IL: W.B. Conkey Co 1900

Lu S, Shen X, and Chen B. (2017). Development of an Efficient Vector System for Gene Knock-out and 
near in-Cis Gene Complementation in the Sugarcane Smut Fungus. Sci Rep-UK 7(1):3113 https://doi.org/10.1038/s41598-017-03233-7

Ma K-W, Ma W. (2016). Phytohormone pathways as targets of pathogens to facilitate infection. Plant Mol. Biol.91: 713-725. doi: $\underline{10.1007 / \mathrm{s} 11103-016-0452-0}$

Maeder DL, Anderson I, Brettin TS, Bruce DC, Gilna P, Han CS, Lapidus A, Metcalf MW, Saunders E, Tapia R, Sowers KR. (2006). The Methanosarcina barkeri genome: comparative analysis with Methanosarcina acetivorans and Methanosarcina mazei reveals extansive rearrangement within Methanosarcinal genomes. J Bacteriol 188: 7922-7931 https://doi.org/10.1128/JB.00810-06

Majumdar S, Zhao P, Pfister NT, Compton M, Olson S, Glover III CVC, Wells L, Graveley BR, Terns RM, Terns MP. (2015). Three CRISPR-Cas9 immune effector complexes coexist in Pyrococcus furiosus. RNA 21(6): 1147-1158 https://doi.org/10.1261/rna.049130.114

Mali P, Yang L, Esvelt KM, Aach J, Guell M, DiCarlo JE, Norville JE, and Church GM. (2013). RNA-Guided Human Genome Engineering via Cas9. Science 339(6121):823-6 https://doi.org/10.1126/science. 1232033

Manica A, Schleper C. (2013). CRISPR-mediated defense mechanisms in the hyperthermophilic archaeal genus Sulfolobus. RNA Biol 10(5):671-678 https://doi.org/10.4161/rna.24154

Martin M. (2011). Cutadapt removes adapter sequences from highthroughput sequencing reads. EMBnet.journal 17(1): 10-12 https://doi.org/10.14806/ej.17.1.200

Marzin S, Hanemann A, Sharma S, Hensel G, Kumlehn J, Schweizer G, Marion SR. (2016). Are PECTIN ESTERASE INHIBITOR genes involved in mediating resistance to Rhynochosporium commune in Barley? PLOS ONE. 11(3): e0150485 https://doi.org/10.1371/journal.pone.0150485

Matsu-ura T, Baek M, Kwon J, Hong C. (2015). Efficient gene editing in Neurospora crassa with CRISPR technology. Fungal Biol Biotech 2:4. https://doi.org/10.1186/s40694-015-0015-1

McDonald JH, Kreitman M. (1991). Adaptive protein evolution at the Adh locus in Drosophila. Nature, $351: 652-654$

McKenna A, Hanna M, Banks E, Sivachenko A, Cibulskis K, Kernytsky A, Garimella K, Altshuler D, 
Gabriel S, Daly M, DePristo MA. (2010). The Genome Analysis Toolkit: a MapReduce framework for analyzing next-generation DNA sequencing data. Genome Res. 20(9): 1297-1303

Mefferd AL, Kornepati AVR, Bogerd HP, Kennedy EM, and Cullen BR. (2015). Expression of CRISPR/Cas Single Guide RNAs Using Small TRNA Promoters. RNA 21(9): 1683-9 https://doi.org/10.1261/rna.051631.115

Meile L, Croll D, Brunner PC, Plissonneau C, Hartmann FE, McDonald BA, Sánchez-allet A. (2018). A fungal avirulence factor encoded in a highly plastic genomic region triggers partial resistance to Septoria tritici blotch. New Phytologist. 219(3): 1048-1061

Meng Y, Hou Y, Wang H, Ji R, Liu B, Wen J, Niu L, Lin H. (2017). Targeted mutagenesis by CRISPR/Cas9 system in the model legume Medicago truncatula. Plant Cell Rep 36(2):371-374. https://doi.org/10.1007/s00299-016-2069-9

Miki D, Zhang W, Zeng W, Feng Z, Jian-Kang Z. (2018). CRISPR/Cas9-mediated gene targeting in Arabidopsis using sequential transformation. Nat Commun 9: 1967 https://doi.org/10.1038/s41467-018-04416-0

Miya A, Albert P, Shinya T, Desaki Y, Ichimura K, Shirasu K, Narusaka Y, Kawakami N, Kaku H, Shibuya N. (2007). CERK1, a LysM receptor kinase, is essential for chitin elicitor signaling in Arabidopsis. Proc. Natl. Acad. Sci. U.S.A. 104: 19613-19618 https://doi.org/10.1073/pnas.0705147104

Mizoi J, Shinozaki K, Yamaguchi-Shinozaki K. (2012). AP2/ERF family transcription factors in plant abiotic stress responses. Biochim. Biophys. Acta. 1819: 86-96.

Mojica FJ, Díez-Vilaseñor C, García-Martínez J, Soria E. (2005). Intervening sequences of regularly spaced prokaryotic repeats derive from foreign genetic elements. J Mol Evol 60(2):174-182. https://doi.org/10.1007/s/00239-004-0046-3

Mojica FJ, Díez-Villaseñor C, Soria E, Juez G. (2000). Biological significance of a family of regularly spaced repeats in the genomes of Archaea, Bacteria and mitochondria. Mol Microbiol 36(1):244246. https://doi.org/10.1046/j.1365-2958.2000.01838.x

Mojica FJM, Juez G, Rodriguez-Valera F. (1993). Transcription at different salinities of Haloferax mediterranei sequences adjacent to partially modified PstI sites. Mol Microbiol 9(3). 
https://doi.org/10.1111/j.1365-2958.1993.tb01721.x

Morran LT, Schmidt OG, Gelarden IA, Parrish II RC, Lively CM. (2011). Running with the Red Queen: Host-parasite coevolution selects for biparental sex. Science. 333(6039): 216-218

Monod M, Capoccia S, Léchenne B, Zaugg C, Holdom M, Jousson O. (2002). Secreted proteases from pathogenic fungi. International Journal of Medical Microbiology 292(5-6): 405-19 https://doi.org/10.1078/1438-4221-00223

Mueller D. (2013). Corn disease loss estimates from the United States and Ontario, Canada. 2012 Purdue University BP-96-12-W

Muller HJ. (1964). The relation of recombination to mutational advance. Mutat. Res. 1: 2-9.

Nakayama T, Blitz IL, Fish MB, Odeleye AO, Manohar S, Cho KWY, Grainger RM. (2014). Chapter Seventeen - Cas9-based genome editing in Xenopus tropicalis. Met Enzymology. 546: 355-375 https://doi.org/10.1016/B978-0-12-801185-0.00017-9

Nayak DD and Metcalf MW. (2017). Cas9-mediated genome editing in the methanogenic archaeon Methanosarcina acetivorans. PNAS 114(11): 2976-2981 https://doi.org/10.1073/pnas.1618596114

Newman M-A, Sundelin T, Nielsen JT, Erbs G. (2013). MAMP (microbe-associated molecular pattern) triggered immunity in plants. Frontiers of Plant Science. https://doi.org/10.3389/fpls.2013.00139

Nickel L, Weidenbach K, Jäger D, Backofen R, Lange SJ, Heidrich N, Schmitz RA. (2013). Two CRISPR-Cas9 systems in Methanosarcina mazei strain Gö1 display common processing features despite belonging to different types I and III. RNA Biol. 10(5): 779-791 https://doi.org/10.4161/rna.23928

Nødvig CS, Nielsen JB, Kogle ME, Mortensen UH. (2015). A CRISPR-Cas9 system for genetic engineering of filamentous fungi. PLOS ONE https://doi.org/10.1371/journal.pone.0133085

Ochiai H. (2015). Single-Base Pair Genome Editing in Human Cells by Using Site-Specific Endonucleases. Int J Mol Sci 16(9):21128-37 https://doi.org/10.3390/ijms160921128.

O'Geen H, Ren C, Nicolet CM, Perez AA, Halmai J, Le VM, MacKay JP, Farnham PJ, and Segal DJ. (2017). dCas9-Based Epigenome Editing Suggests Acquisition of Histone Methylation Is Not Sufficient for Target Gene Repression. Nucleic Acids Res 45(17):9901-16 https://doi.org/10.1093/nar/gkx578 
Ozaki A, Konishi R, Otomo C, Kishida M, Takayama S, Matsumoto T, Tanaka T, Kondo A. (2017). Metabolic engineering of Schizosaccharomyces pombe via CRISPR-Cas9 gneome editing for lactic acid production from glucose and cellobiose. Metab Eng Commun https://doi.org/10.1016/j.meteno.2017.08.002

Pawluk A, Amrani N, Zhang Y, Garcia B, Hidalgo-Reyes Y, Lee J, Edraki A, Megha S, Sontheimer EJ, Maxwell KL, Davidson AR. (2016). Naturally Occurring Off- Switches for CRISPR-Cas9. Cell 167(7): 1829-38 https://doi.org/10.1016/j.cell.2016.11.017

Perlin MH, Beckerson WC, Gopinath A, Cobbs GA. (2020). Molecular and Cellular Genetics: laboratory Studies $2^{\text {nd }}$ Edition. San Diego, CA: Cognella Academic Publishing.

Perlin MH, Amselem J, Fontanillas E, Toh SS, Chen Z, Goldberg J, Duplessis S, Henrissat B, Young S, Zeng Q, Aguileta G, Petit E, Badouin H, Andrews J, Razeeq D, Gabaldón T, Quesneville H, Giraud T, Hood ME, Schultz DJ, Cuomo CA. (2015). Sex and parasites: genomic and transcriptomic analysis of Microbotryum lychnidis-dioicae, the biotrophic and plant-castrating anther smut fungus. BMC Genomics. 16(461): 1-24

Perlin MH, Hughes C, Welch J, Akkaraju S, Steinecker D, Kumar A, Smith B, Garr SS, Brown SA, Ando T. (1997). Molecular approaches to differentiate subpopulations or formae speciales of the fungal phytopathogen Microbotryum violaceum. Int J Mol Sci. 158 (5): 568-574

Petersen TN, Brunak S, von Heijne G, Nielsen H. (2011). SignalP 4.0: discriminating signal peptides from transmembrane regions. Nature Methods. 8: 785-786

Pevsner J. (2015). Bioinformatics and Functional Genomics. Hoboken, NJ: John Wiley \& Sons.

Pfeifer B, Wittelsbürger U, Ramos-Onsins S E, Lercher M J. (2014). POPGENOME: an efficient Swiss army knife for population genomic analyses in R. Molecular Biology and Evolution, 31, 19291936 Pierleoni A, Martelli PL, Casadio R. 2008. PredGPI: a GPI-anchor predictor. BMC Bioinformatics. 23(9): 392

Plissonneau C, Stürchler A, Croll D. (2016). The evolution of orphan regions in genomes of a fungal pathogen of wheat. MBio. 7(5). pii: e01231-16

Plissonneau C, Hartmann FE, Croll D. (2018). Pangenome analyses of the wheat pathogen Zymoseptoria tritici reveal the structural basis of a highly plastic eukaryotic genome. BMC Biol. 16(1): 5 
Port F, Strein C, Stricker M, Rauscher B, Heigwer F, Zhou J, Beyersdörffer C, Frei J, Hess A, Kern K, Malamud R, Pavlovic B, Rädecke K, Schmitt L, Voos L, Valentini E, Boutros M. (2019). A large-scale resource for tissue-specific CRISPR mutagenesis in Drosphila. BioRxiv https://doi.org/10.1101/636076

Presnell JS, Browne WE. (2019). Krüppel-like factor gene function in the ctenophore mnemiopsis suggest an ancient role in promoting cell proliferation in metazoan stem cell niches. BioRxiv https://dx.doi.org/10.1101/527002

Price MA, Cruz R, Baxter S, Escalettes F, Rosser SJ. (2019). CRISPR-Cas9 In Situ engineering of subtilisin E in Bacillus subtilis. PLOS One https://doi.org/10.1371/journal.pone.0210121

Quinlan AR, Hall IM. (2010). BEDTools: a flexible suite of utilities for comparing genomic features. Bioinformatics. 26(6):841-2 https://doi.org/10.1093/bioinformatics/btq033

R Core Team. (2019). R: A language and environment for statistical computing. R Foundation for Statistical Computing, Vienna, Austria. https:/www.R-project.org/

Rabe, F., Ajami-Rashidi, Z., Doehlemann, G., Kahmann, R., and Djamei, A. (2013). Degradation of the plant defence hormone salicylic acid by the biotrophic fungus Ustilago maydis. Mol Microbiol 89: $179-188$.

Rasys AM, Park S, Ball RE, Alcala AJ, Lauderdale JD, Menke DB. (2019). CRISPR-Cas9 gene editing in lizards through microinjection of unfertilized oocytes. BioRxiv https://dx.doi.org/10.1101/591446

Refrégier G, Gac ML, Jabbour F, Widmer A, Shykoff J, Yockteng R, Hood ME, Giraud T. (2008). Cophylogeny of the anther smut fungi and their Caryophyllaceous hosts: Prevalence of host shifts and importance of delimiting parasite species for inferring cospeciation. BMC Evolutionary Biology. 8(100) https://doi.org/10.1186/1471-2148-8-100

Reich, C., Wise, J. A. (1990). Evolutionary origin of the U6 small nuclear RNA intron. Mol Cell Biol 10(10):5548-52 https://doi.org/10.1128/MCB.10.10.5548

Reis H, Pfiffi S, Hahn M. (2005). Molecular and functional characterization of a secreted lipase from Botrytis cinerea. Mol Plant Pathol. 6(3): 257-67

https://doi.org/10.1111/j.1364-3703.2005.00280.x 
Rep M. (2005). Small proteins of plant-pathogenic fungi secreted during host colonization. FEMS Microbiology Letters. 253(1): 19-27 https://doi.org/10.1016/j.femsle.2005.09.014

Richter H, Zoephel J, Schermuly J, Maticzka D, Backofen R, Randau L. (2012). Characterization of CRISPR RNA processing in Clostridium thermocellum and methanococcus mariplaludis. Nucleic Acids Res 40(19): 9887-9896 https://doi.org/10.1093/nar/gks737

Richardson CD, Kazane KR, Feng SJ, Zelin E, Bray NL, Schäfer AJ, Floor SN, Corn JE. (2010). CRISPRCas9 genome editing in human cells occurs via the Fanconi anemia pathway. Nat Gen 50: 11321139. https://doi.org/10.1038/s41588-018-0174-0

Robson RL, Jones R, Robson RM, Schwartz A, Richardson TH. (2015). Azotobacter genomes: the genome of Azotobacter chroococcum NCIMB 8003 (ATCC 4412). PLOS ONE https://doi.org/10.1371/journal.pone.0127997

Sánchez-Vallet A, Fouché S, Fudal I, Hartmann FE, Soyet JL, Tellier A, Croll D. (2018). The genome biology of effector gene evolution in filamentous plant pathogens. Annu Rev Phytopathol. 56: $21-40$

Sanchez L, Courteaux B, Hubert J, Kauffmann S, Renault JH, Clement C, Baillieul F, Dorey S. (2012). Rhamnolipids elicit defense responses and induce disease resistance against biotrophic, hemibiotrophic, and necrotrophic pathogens that require different signaling pathways in Arabidopsis and highlight a central role for salicylic acid. Plant Physiol. 160: 1630-1641 https://doi.org/10.1104/pp.112.201913

Sanders SM, Ma Z, Hughes JM, Riscoe BM, Givson GA, Watson AM, Flici H, Frank U, Schnitzler CE, Baxevanis AD, Nicotra ML. (2018). CRISPR/Cas9-mediated gene knockin in the hydroid Hydractinia symbiolongicarpus. BMC Genomics 19: 649 https://doi.org/10.1186/s12864-018-5032-z

Savchenko T, Walley JW, Chehab EW, Xiao Y, Kaspi R, Pye MF, Mohamed ME, Lazarus CM, Bostock RM, Dehesh K. (2010). Arachidonic acid: an evolutionarily conserved signaling molecule modulates plant stress signaling networks. Plant Cell. 22(10): 3193-3205 https://doi.org/10.1105/tpc.110.073858

Schäfer AM, Kemler M, Bauer R, Begerow D. (2010). The illustrated life cycle of Microbotryum on the 
host plant Silene latifolia. Botany. 88: 875-885.

Schirawski J. et.al. 2010. Pathogenicity determinants in smut fungi revealed by genome comparison. Science. 330(6010): 1546-1548

Scholz I, Lange SJ, Hein S, Hess WR, Backofen R. (2013). CRISPR-Cas systems in the Cyanobacterium Synechocystis sp. PCC6803 exhibit distinct processing pathways involving at least two Cas6 and Cmr2 protein. PLOS ONE https://doi.org/10.1371/journal.pone.0056470

Schuster M, Kahmann R. (2019). CRISPR-Cas9 genome editing approaches in filamentous fungi and oomycetes. Fungal Genet Biol. 130: 43-53 https://doi.org/10.1016/j.fgb.2019.04.016

Schuster M, Schweizer G, Kahmann R. (2018). Comparative analyses of secreted proteins in plant pathogenic smut fungi and related basidiomycetes. Fungal Genet Biol. 112: 21-30

Schuster M, Schweizer G, and Kahmann R. (2016). Genome editing in Ustilago maydis using the CRISPRCas system. Fungal Genet and Biol 89: 3-9. https://doi.org/10.1016/j.fgb.2015.09.001

Sekine R, Kawata T, and Muramoto T. (2018). CRISPR/Cas9 Mediated Targeting of Multiple Genes in Dictyostelium. Sci Rep-UK 8(1): 8471 https://doi.org/10.1038/s41598-018-26756-z

Sidik SM, Huet D, Lourido S. (2018). CRISPR-Cas9-based genome-wide screening of Toxoplasma gondii. Nat Protoc 13(1): 307-323 https://doi.org/10.1038/nprot.2017.131

Sorlien EL, Witucki MA, Ogas J. (2018). Efficient production and identification of CRISPR/Cas9generated gene knockouts in the model system Danio rerio. J Vis Exp (138), e56969. https://doi.org/10.3791/56989

Sperschneider J, Gardiner DM, Dodds PN, Tini F, Covarelli 1, Singh KB, Manners JM, Taylor JM. (2015). EffectorP: Predicting fungal effector proteins from secretomes using machine learning. New Phytologist. https://doi.org/10.1111/nph.13794

Square T, Romášek M, Jandzik D, Cattell MV, Klymokowsky M, Medeiros DM. (2015). CRISPR/Cas9-mediated mutagenesis in the sea lamprey Petromyzon marinus: a powerful tool for understanding ancestral gene functions in vertebrates. Development 142: 4180-4187. https://doi.org/10.1242/dev.125609

Stachler A-E, Marchfelder A. (2016). Gene repression in Haloarchaea using the CRISPR (Clustered Regularly Interspaced Short Palindromic Repeats)-Cas I-B system. J Biol Chem 291(29): 15226- 
15242 https://doi.org/10.1074/jbc.M116.724062

Stern A, Doron-Faigenboim A, Erez E, Martz E, Bacharach E, Pupko T. (2007). Advanced models for detecting positive and purifying selection using a Bayesian inference approach. Nucleic Acids Res. 35: W506-11.

Stolfi A, Gandhi S, Salek F, Christiaen L. (2014). Tissue-specific genome editing in Ciona embryos by CRISPR/Cas9. Development 141(21): 4115-20 https://doi.org/10.1242/dev.114488

Strobel G. (2018). The Emergence of Endophytic Microbes and their Biological Promise. Journal of Fungi. 4(2): 57

Sugano SS, Nishihama R, Shirakawa M, Takagi J, Matsuda Y, Ishida S, Shimada T, hara-Nishimura I, Osakabe K, Kohchi T. (2018). Efficient CRISPR/Cas9-based genome editing and its application to conditional genetic analysis in Marchantia polymorpha. PLOS ONE https://doi.org/10.1371/journal.pone.0205117

Sun D, Guo Z, Liu Y, Zhang Y. (2017). Progress and prospects of CRISPR/Cas9 systems in insects and other arthropods. Front Physiol https://doi.org/10.3389/fphys.2017.00608

Tan SZ, Reisch CR, Prather KLJ. (2018). A robust CRISPR interference gene repression system in Pseudomonas. J Bacteriol 200(7):1-12 https://doi.org/10.1128/JB.00575-17

Taning CNT, Van Eynde B, Yu N, Ma S, Smagghe G. (2017). CRISPR/Cas9 in insects: applications, best practices and biosafety concerns. J Insect Physiol 98: 245-257. https://doi.org/10.1016/j.jinphys.2017.01.007

Tao W, Yang A, Deng Z, Sun Y. (2018). CRISPR/Cas9-based editing of Streptomyces for discovery, characterization, and production of natural products. Front Microbiol 9: 1660. https://doi.org/10.3389/fmicb.2018.01660

Testa AC, Oliver AP, Hane JK. (2016). OcculterCut: A Comprehensive Survey of AT-Rich Regions in Fungal Genomes. Genome Biol Evol. 8(6): 2044-64 https://doi.org/10.1093/gbe/evw121

Thrall PH. (2016). Epidemiological and evolutionary outcomes in gene-for-gene and matching allele models. Frontiers in Plant Science. 6: 1084 https://doi.org/10.3389/fpls.2015.01084

Toh SS, Chen Z, Rouchka EC, Schultz DJ, Cuomo CA, Perlin MH. (2018). Pas de deux: An intricate dance 
of anther smut and its host. G3: Genes, Genomes, Genetics| 8:2 505-518;

https://doi.org/10.1534/g3.117.300318

Toh SS, Chen Z, Schultz DJ, Cuomo CA, Perlin MH. (2017). Transcriptional analysis of mating and preinfection stages of the anther smut, Microbotryum lychnidis-dioicae. Microbiology. 163: 410-420.

Toh SS, Treves DS, Barati MT, and Perlin MH. (2016). Reliable Transformation System for Microbotryum Lychnidis-Dioicae Informed by Genome and Transcriptome Project. Arch Microbiol 198(8): 813-25 https://doi.org/10.1007/s00203-016-1244-2

Toh SS, Perlin MH. (2015). Size does matter: staging of Silene latifolia floral buds for transcriptome studies. Int J Mol Sci 16: 22027-22045.

Tsuda K, Katagiri F. (2010). Comparing signaling mechanisms engaged in pattern-triggered and effectortriggered immunity. Current Opinions in Plant Biology. 13(4): 459-65. https://doi.org/10.1016/j.pbi.2010.04.006

Tu Q, Yin J, Fu J, Herrmann J, Li Y, Yin Y, Stewart AF, Müller R. (2016). Room temperature electrocompetent bacterial cells improve DNA transformation and recombineering efficiency. Sci Rep-UK 6; 246-48. https://doi.org/10.1038/srep24648

Urry LA, Cain ML, Wasserman SA, Minorsky PV, Reece JB. (2017). Campbell Biology AP Edition, $11^{\text {th }}$ Edition. NY: Pearson.

USDA. (2017). Agricultural Research Service: Cereal Disease Lab: St. Paul, MN https://www.ars.usda.gov/midwest-area/stpaul/cereal-disease-lab/docs/cereal-rusts/wheat-stemrust/

Van de Wouw AP, Cozijnsen AJ, Hane JK, Brunner PC, McDonald BA, Oliver RP, Howlett BJ. (2010). Evolution of linked avirulence effectors in Leptosphaeria maculans is affected by genomic environment and exposure to resistance genes in host plants. PLoS Pathog. 4;6(11): e1001180 https://doi.org/10.1371/journal.ppat.1001180

Van Valen L. (1973). A new evolutionary law. Evolution Theory. 1: 1-30

Vienne DM, Refrégier G, López-Villavicencio M, Tellier A, Hood ME, Giraud T. (2013). Cospeciation vs host-shift speciation: methods for testing, evidence from natural associations and relation to coevolution. New Phytol. 198: 347-385 
Vincent D, Rafiqui M, Job D. (2020). The multiple facets of plant-fungal interactions reveled through plant and fungal secretomics. Frontiers in Plant Science. 10(1626): 1-19

Vonk PJ, Escobar N, Wösten HAB, Lugones LG, Ohm RA. (2019). High-throughput targeted gene deletion in the model mushroom Schizophyllum commune using pre-assembled Cas9 ribonucleoptroteins. Sci Rep-UK 9:7632 https://doi.org/10.1038/s41598-019-44133-2

Wang F, Sun X, Shi X, Zhai H, Tian C, Kong F, Liu B, Yuan X. (2016). A global analysis of the polygalacturonase gene family in soybean (Glycine max). PLoS One. 11(9):e0163012 https://doi.org/10.1371/journal.pone.0163012

Wang L, Deng A, Zhang Y, Liu S, Liang Y, Bai H, Cui D, Oiu Q, Shang X, Yang Z, He X, Wen T. (2018). Efficient CRISPR-Cas9 mediated multiplex genome editing in yeast. Biotechnol Biofuels 11: 277 https://doi.org/10.1186/s13068-018-1271-0

Wang L, Wang L, Tan Q, Fan Q, Zhu H, Hong Z, Zhang Z, Duanmu D. (2016). Efficient inactivation of symbiotic nitrogen fixation related genes in Lotus japonicus using CRISPR-Cas9. Front Plant Sci 7:1333 https://doi.org/10.3389/fpls.2016.01333

Watters KE, Fellmann C, Bai HB, Ren SM, and Doudna JA. (2018). Systematic Discovery of Natural CRISPR-Cas12a Inhibitors. Science 362(6411): 236-9 https://doi.org/10.1126/science.aau5138

Wei Y, Qiu Y, Chen Y, Liu G, Zhang Y, Xu L, and Ding Q. (2017). CRISPR/Cas9 with Single Guide RNA Expression Driven by Small TRNA Promoters Showed Reduced Editing Efficiency Compared to a U6 Promoter. RNA 23(1)1-5 https://doi.org/10.1261/rna.057596.116

Wei W, Xin H, Roy B, Dai J, Miao Y, Gao G. (2014). Heritable genome editing with CRISPR/Cas9 in the silkworm, Bombyx mori. PLOS ONE https://doi.org/10.1371/journal.pone.0101210

Whisson SC, Boevink PC, Moleleki L, Avrova AO, Morales JG, Gilroy EM, Armstrong MR, Grouffaud S, van West P, Chapman S, Hein I, Toth IK, Pritchard L, Birch PR. (2007). A translocation signal for delivery of oomycete effector proteins into host plant cells. Nature. 450: 115-118

Whittle CA, Votintseva A, Ridout K, Filatov DA. (2015). Recent and massive expansion of the matingtype-specific region in the smut fungus Microbotryum. Genetics, 199(3): 809-816.

Willmann R, Lajunen HM, Erbs G, Newman MA, Kolb D, Tsuda K, Katagiri F, Fliegmann J, Bono JJ, 
Cullimore JV, Jehle AK, Götz F, Kulik A, Molinaro A, Lipka V, Gust AA, Nürnberger T. (2011). Arabidopsis lysin-motif proteins LYM1 LYM3 CERK1 mediate bacterial peptidoglycan sensing and immunity to bacterial infection. Proc. Natl. Acad. Sci. U.S.A. 108: 19824-19829.

https://doi.org/10.1073/pnas.1112862108

Witte H, Moreno E, Rödelsperger C, Kim J, Kim J-S, Streit A, Sommer RJ. (2015). Gene inactivation using the CRISPR/Cas9 system in the nematode Pristionchus pacificusi. Dev Genes Evol 225(1): 55-62 https://doi.org/10.1007/s00427-014-0486-8

Wu R, Lucke M, Jang Y, Zhu W, Symeonidi E, Wang C, Fitz J, Xi W, Schwab R, Weigel D. (2018). An efficient CRISPR vector toolbox for engineering large deletions in Arabidopsis thaliana. Plant Methods 14: 65 https://doi.org/10.1186/s13007-018-0330-7

Wu X, Kriz AJ, and Sharp PA. (2014). Target Specificity of the CRISPR-Cas9 System. Quantitative Biol 2(2): 59-70 https://doi.org/10.1007/s40484-014-0030-x

Westbrook AW, Moo-Young M, Chou CP. (2018). Development of a CRISPR-Cas9 tool kit for comprehensive engineering of Bacillus subtilis. Appl Environ Microb 82(16): 4876-4895 https://doi.org/10.1128/AEM.01159-16

Xiao Y, Wang S, Rommelfanger S, Balassy A, Barba-Ostria C, Gu P, Galazka JM, Zhang F. (2018). Developing a Cas9-based tool to engineer native plasmids in Synechocystis sp. PCC 6803. Biotechnol Bioeng 115(9): 2305-2314 https://doi.org/10.1002/bit.26747

Xie C, Chen YL, Wang DF, Wang YL, Zhang TP, Li H, Liang F, Zhao Y, and Zhang GY. (2017). SgRNA Expression of CRIPSR-Cas9 System Based on MiRNA Polycistrons as a Versatile Tool to Manipulate Multiple and Tissue-Specific Genome Editing. Sci Rep-UK 7(1): 5795 https://doi.org/10.1038/s41598-017-06216-w

Xin LUO, Min LI, Bing SU. (2016). Application of the genome editing tool CRISPR/Cas9 in nonhuman primates. Zool Research 37(4): 241-219. doi: 10.13918/j.issn.2095-8137.2016.4.214

Xu J, Lian W, Jia Y, Li L, and Huang Z. (2017). Optimized guide RNA structure for genome editing via Cas9. Oncotarget 8(55): 94166-94171 https://doi.org/10.18632/oncotarget.21607

Yang L, Mali P, Kim-Kiselak C, Church G. (2014). CRISPR-Cas-mediated targeted genome editing in human cells. Methods Mol. Biol. 1114:245-267 https://doi.org/10.1007/978-1-62703-761-7_16 
Yang Z, Nielsen R, Goldman N, Perdersen AM. (2000). Codon-substitution models for heterogeneous selection pressure at amino acid sites. Genetics. 155(1): 431-449

Zeaiter Z, Mapelli F, Crotti E, Borin S. (2018). Methods for the genetic manipulation of marina bacteria Electron J Biotechn 33: 17-28. https://doi.org/10.1016/j.ejbt.2018.03.003

Zebec Z, Manica A, Zhang J, White MF, Schleper C. (2014). CRISPR-mediated targeted mRNA degradation in the archaeon Sulfolobus solfataricus. Nucleic Acids Res 42(8): 5280-4288. https://doi.org/10.1093/nar/gku161

Zhang T, Gao Y, Wang R, and Zhao Y. (2017). Production of Guide RNAs in Vitro and in Vivo for CRISPR Using Ribozymes and RNA Polymerase II Promoters. BIO-PROTOCOL 7(4)e2148 https://doi.org/10.21769/BioProtoc. 2148

Zhang Z, Zhang Y, Gao F, Han S, Cheah KS, Tse H-F, Lian Q. (2017). CRISPR/Cas9 genome-editing system in human stem cells: current status and future prospects. Mol Ther-Nucl Acids. 9:230-241. https://doi.org/10.1016/j.omtn.2017.09.009

Zheng K, Wang Y, Li N, Jiang F-F, Wu C-X, Liu F, Chen, H-C, Liu, Z-F. (2018). Highly efficient base editing in bacteria using a Cas9-cytidine deaminase fusion. Commun. Biol. 1: 32

Zuo Q, Wang Y, Cheng S, Lian C, Tang B, Wang F, Lu Z, Ji Y, Zhao R, Zhang W, Jin K, Song J, Zhang Y, Li B. (2016). Site-directed genome knockout in chicken cell line and embryos can use CRISPR/Cas gene editing technology. G3 (Bethesda) 6(6): 1787-1792.

https://doi.org/10.1534/g3.116.028803 


\section{APPENDIX}

\section{Supplemental Material:}

Supplemental Table C3-1

\begin{tabular}{|c|c|c|c|c|}
\hline Microbotryum & Nucleotide Sequence & $\begin{array}{l}\text { \# bp } \\
\text { Sub. }\end{array}$ & Amino Acid Sequence & $\begin{array}{l}\# \text { aa } \\
\text { Sub. }\end{array}$ \\
\hline MVLG & $\begin{array}{l}\text { ATGACCTCACAAGTGCGAA } \\
\text { TGCAAGTCGAGAGTCGTG } \\
\text { CCCAACGACGCGCAGGGG } \\
\text { CCTACGCGTCCATGAGGTT } \\
\text { GTTGCTCGCTCTGGTCTTC } \\
\text { GCCCTCTGCACCTTAGCG } \\
\text { CACCTGCCGACAACCAGT } \\
\text { GCCGCACCGCTGGCTTCG } \\
\text { GAGCAAATCTCGTCCGGT } \\
\text { CTCGTCTTTCGACAAGAAC } \\
\text { CACCCAGATGGTTACAATT } \\
\text { CTCTCGGCCTCATGAGAAA } \\
\text { GTGTCGCATCAAGGCAAA } \\
\text { GATCATCTGGACTGGAAAA } \\
\text { ACACGTCGCCTTCGCCGTT } \\
\text { CACTTCCAGCGAACCATCG } \\
\text { AGGCGTGTGAAACGTGAC } \\
\text { GAGATGTGGGAGCAGTAC } \\
\text { ATCGAGGGGGATGAGATC } \\
\text { GACGGGGAGAAGAGCGAG } \\
\text { GATGTTCGTGCAGGAGAT } \\
\text { CCGGATGTTGCCGGGGAT } \\
\text { GAAGTCCTGACAGACACC } \\
\text { GAGATCGCGGGCGGAGCG } \\
\text { GACGAAGCGGGCGAGGG } \\
\text { GTCCACAGGGGAAAAGTG } \\
\text { GTGGCAAGCCAGGAGACG } \\
\text { ATTGCGTGAGAGGCGATC } \\
\text { GGCCACCACAAGGGTTGT } \\
\text { TCCGTAACGGCTCTTTGTT } \\
\text { C }\end{array}$ & & $\begin{array}{l}\text { MTSQVRMQVESRAQRRA } \\
\text { GAYASMRLLLALVFALCTL } \\
\text { AHLPTTSAAPLASEQISSG } \\
\text { LVFRQEPPRWLQFSRPHE } \\
\text { KVSHQGKDHLDWKNTSP } \\
\text { SPFTSSEPSRRVKRDEM } \\
\text { WEQYIEGDEIDGEKSEDV } \\
\text { RAGDPDVAGDEVLTDTEI } \\
\text { AGGADEAGEGSTGEKW } \\
\text { WQARRRLRERRSATTRV } \\
\text { VP* }^{*}\end{array}$ & \\
\hline MvSI & $\begin{array}{l}\text { ATGACCTCACAAGTGCGAA } \\
\text { TGCAAGTCGAGAGTCGTG } \\
\text { CCCAACGACGCGCAGGGG } \\
\text { CCTACGCGTCCATGAGGTT } \\
\text { GTTGCTCGCTCTGGTCTTC } \\
\text { GCCCTCTGCACCTTAGCG } \\
\text { CACCTGCCGACAACCAGT } \\
\text { GCCGCACCGCTGGCTTCG }\end{array}$ & 0 & $\begin{array}{l}\text { MTSQVRMQVESRAQRRA } \\
\text { GAYASMRLLLALVFALCTL } \\
\text { AHLPTTSAAPLASEQISSG } \\
\text { LVFRQEPPRWLQFSRPHE } \\
\text { KVSHQGKDHLDWKNTSP } \\
\text { SPFTSSEPSRRVKRDEM } \\
\text { WEQYIEGDEIDGEKSEDV } \\
\text { RAGDPDVAGDEVLTDTEI }\end{array}$ & 0 \\
\hline
\end{tabular}




\begin{tabular}{|c|c|c|c|c|}
\hline & $\begin{array}{l}\text { GAGCAAATCTCGTCCGGT } \\
\text { CTCGTCTTTCGACAAGAAC } \\
\text { CACCCAGATGGTTACAATT } \\
\text { CTCTCGGCCTCATGAGAAA } \\
\text { GTGTCGCATCAAGGCAAA } \\
\text { GATCATCTGGACTGGAAAA } \\
\text { ACACGTCGCCTTCGCCGTT } \\
\text { CACTTCCAGCGAACCATCG } \\
\text { AGGCGTGTGAAACGTGAC } \\
\text { GAGATGTGGGAGCAGTAC } \\
\text { ATCGAGGGGGATGAGATC } \\
\text { GACGGGGAGAAGAGCGAG } \\
\text { GATGTTCGTGCAGGAGAT } \\
\text { CCGGATGTTGCCGGGGAT } \\
\text { GAAGTCCTGACAGACACC } \\
\text { GAGATCGCGGGCGGAGCG } \\
\text { GACGAAGCGGGCGAGGG } \\
\text { GTCCACAGGGGAAAAGTG } \\
\text { GTGGCAAGCCAGGAGACG } \\
\text { ATTGCGTGAGAGGCGATC } \\
\text { GGCCACCACAAGGGTTGT } \\
\text { TCCGTAACGGCTCTTTGTT } \\
\text { C }\end{array}$ & & $\begin{array}{l}\text { AGGADEAGEGSTGEKW } \\
\text { WQARRRLRERRSATTRV } \\
\text { VP* }\end{array}$ & \\
\hline MvSd & $\begin{array}{l}\text { ATGACCTCACAAGTGCGAA } \\
\text { TGCAAGTCGAGAGTCGTG } \\
\text { CCCAACGACGCGCAGGGG } \\
\text { CCTACGCGTCCATGAGGTT } \\
\text { GTTGCTCGCTCTGGTCTTC } \\
\text { GCCCTCTGCACCCTAGCG } \\
\text { CACCTGCCGACAACCAGT } \\
\text { GCCGCACCGCTGGCTTCG } \\
\text { GAGCAAATCTCGTCCGGT } \\
\text { CTCGTCTTTCGACAAGAAC } \\
\text { CACCCAGATGGTTACAATT } \\
\text { CTCTCGGCCTCATGAGAAA } \\
\text { GTGTCGCATCAAGACAAAG } \\
\text { ATCATCTGGACTGGAAAAA } \\
\text { CACGTCGCCTTCGCCGTT } \\
\text { CACTTCCAGCGAACCATCG } \\
\text { AGGCGTGTGAAAGTGAC } \\
\text { GAGATGTGGGAGCAGTAC } \\
\text { ATCGAGGGGGATGAGATC } \\
\text { GACGGGGAGAAGAGCGAG } \\
\text { GAAGTTCGTGCAGGAGAT } \\
\text { CCGGATGTTGCCAGGGAT } \\
\text { GAAGTTCTGACAGACACC } \\
\text { GAGATCGCGGGCGAAGCG } \\
\text { GACGAAGCGGGCGAGGG }\end{array}$ & 6 & $\begin{array}{l}\text { MTSQVRMQVESRAQRRA } \\
\text { GAYASMRLLLALVFALCTL } \\
\text { AHLPTTSAAPLASEQISSG } \\
\text { LVFRQEPPRWLQFSRPHE } \\
\text { KVSHQDKDHLDWKNTSP } \\
\text { SPFTSSEPSRRVKRDEM } \\
\text { WEQYIEGDEIDGEKSEEV } \\
\text { RAGDPDVARDEVLTDTEI } \\
\text { AGEADEAGEGSTGEKWW } \\
\text { QARRRLRERRSATTRVVP } \\
\text { * }\end{array}$ & 4 \\
\hline
\end{tabular}




\begin{tabular}{|c|c|c|c|c|}
\hline & $\begin{array}{l}\text { GTCCACAGGGGAAAAGTG } \\
\text { GTGGCAAGCCAGGAGACG } \\
\text { ATTGCGTGAGAGGCGATC } \\
\text { GGCCACCACAAGGGTTGT } \\
\text { TCCGTAACGGCTCTTTGTT } \\
\text { C }\end{array}$ & & & \\
\hline MvSp & $\begin{array}{l}\text { ATGAACTCACAAGCGCGAA } \\
\text { TGCAAGTCGAGAGTCGTG } \\
\text { CCCAACGGCGCGCGGGG } \\
\text { GCCTACACGTCCCAGAGG } \\
\text { TTGTTACTCGCTCTGGTCT } \\
\text { TCGCCCTCTGCACCTTAGC } \\
\text { ACACCTATCGACAACCAGA } \\
\text { GCCGCACCGCTGGCTTCA } \\
\text { GAGCAAATCTCGTCCCGTC } \\
\text { TCGTCTTTCGACAAGAAAC } \\
\text { ACCGCGATGGTTACGATTC } \\
\text { TCTCGCCCTCATGAGAAG } \\
\text { GCCTCGCATCAAGGCAAA } \\
\text { CATCATCTGGACCGGAAAA } \\
\text { ACACGTTGCCTTCGCCGTT } \\
\text { CACTTCCAGCGAACCATCG } \\
\text { AGGCGGGTGAAACGTGAC } \\
\text { GAGATGTGGGAGCAGTAC } \\
\text { ATTGAGGGGGATGAAATC } \\
\text { GACGGGGAGAACAGCGAG } \\
\text { AAACTTGGTCCAGGAGATC } \\
\text { CGGATGTTGCCGAGGATG } \\
\text { AAATTCTGACAAACATCGA } \\
\text { GATCGCGGGCGAAGCTGA } \\
\text { GGAAGCGGGCGAGTGGTC } \\
\text { CATAGGAGAAAAGTGGTG } \\
\text { GCAAGCCAGGAGACGATT } \\
\text { GCGTGAGCGGCGTTTGGC } \\
\text { CACCGCAAAGGTTGTTCC } \\
\text { GTAACGGCGCTTTGTTC }\end{array}$ & 51 & $\begin{array}{l}\text { MNSQARMQVESRAQRRA } \\
\text { GAYTSQRLLLALVFALCTL } \\
\text { AHLSTTRAAPLASEQISSR } \\
\text { LVFRQETPRWLRFSRPHE } \\
\text { KASHQGKHHLDRKNTLPS } \\
\text { PFTSSEPSRRVKRDEMW } \\
\text { EQYIEGDEIDGENSEKLG } \\
\text { PGDPDVAEDEILTNIEIAG } \\
\text { EAEEAGEWSIGEKWWQA } \\
\text { RRRLRERR AATAKVV** }\end{array}$ & 29 \\
\hline
\end{tabular}

Supplemental Table C3-1

\begin{tabular}{|l|l|l|l|}
\hline \multicolumn{1}{|c|}{ Program } & \multicolumn{1}{c|}{ MvSI } & \multicolumn{1}{c|}{ MvSd } & \multicolumn{1}{c|}{ MvSp } \\
\hline SignalP 4.1 & D Score $=0.695$ & D Score $=0.695$ & D Score $=0.412$ \\
\hline EffectorP 1.0 & Prob $=0.865$ & Prob $=0.686$ & Prob $=0.645$ \\
\hline Pfam 32 & Hits $=0$ & Hits $=0$ & Hits $=0$ \\
\hline HMMER 3.1b1 & Hits $=0$ & Hits $=0$ & Hits $=0$ \\
\hline
\end{tabular}




\begin{abstract}
Supplemental File C5-1.
$>$ Cas 9

MDKKYSIGLDIGTNSVGWAVITDEYKVPSKKFKVLGNTDRHSIKKNLIGALLFDSGETAEATRLKRTARRRYTRRKNRICYLQEIFSNE MAKVDDSFFHRLEESFLVEEDKKHERHPIFGNIVDEVAYHEKYPTIYHLRKKLVDSTDKADLRLIYLALAHMIKFRGHFLIEGDLNPDN SDVDKLF IQLVQTYNQLFEENPINASGVDAKAILSARLSKSRRLENLIAQLPGEKKNGLFGNLIALSLGLTPNFKSNFDLAEDAKLQLS KDTYDDDLDNLLAQIGDQYADLFLAAKNLSDAILLSDILRVNTE ITKAPLSASMIKRYDEHHQDLTLLKALVRQQLPEKYKEIFFDQSK NGYAGY IDGGASQEEFYKF I KP ILEKMDGTEELLVKLNREDLLRKQRTF DNGS IPHQI HLGELHAILRRQEDFYPFLKDNREKIEKILT FRIPYYVGPLARGNSRFAWMTRKSEETITPWNFEEVVDKGASAOSF IERMTNFDKNLPNEKVLPKHSLLYEYFTVYNELTKVKYVTEGM RKPAFLSGEQKKAIVDLLFKTNRKVTVKQLKEDYFKKIECFDSVEISGVEDRFNASLGTYHDLLKI IKDKDFLDNEENEDILEDIVLTL TLFEDREMIEERLKTYAHLFDDKVMKQLKRRRYTGWGRLSRKLINGIRDKQSGKTILDFLKSDGFANRNFMQLIHDDSLTFKEDIQKAQ VSGOGDSLHEHIANLAGSPAIKKGILOTVKVVDELVKVMGRHKPENIVIEMARENOTTOKGOKNSRERMKRIEEGIKELGSOILKEHPV ENTQLQNEKLYLYYLQNGRDMYVDQELDINRLSDYDVDH IVPQSFLKDDS IDNKVLTRSDKNRGKSDNVPSEEVVKKMKNYWRQLLNAK LITQRKFDNLTKAERGGLSELDKAGF IKROLVETROITKHVAOILDSRMNTKYDENDKLIREVKVITLKSKLVSDFRKDFOFYKVREIN NYHHAHDAYLNAVVGTALIKKYPKLESEFVYGDYKVYDVRKMIAKSEQE IGKATAKYFFYSN IMNFFKTE ITLANGE IRKRPLIETNGE TGEIVWDKGRDFATVRKVLSMPQVNIVKKTEVQTGGF SKESILPKRNSDKLIARKKDWDPKKYGGFDSPTVAYSVLVVAKVEKGKSKKL KSVKELLGITIMERSSFEKNPIDFLEAKGYKEVKKDLI IKLPKYSLFELENGRKRMLASAGELQKGNELALPSKYVNFLYLASHYEKLK GSPEDNEQKQLFVEQHKHYLDEI IEQISEF SKRVILADANLDKVLSAYNKHRDKPIREQAENI I HLFTLTNLGAPAAFKYFDTTIDRKR YTSTKEVLDATLIHQSITGLYETRIDLSOLGGD
\end{abstract}

$>\operatorname{Cas} 9 \mathrm{n}$ (D10A)

MDKKYSIGLAIGTNSVGWAVITDEYKVPSKKFKVLGNTDRHS IKKNLIGALLFDSGETAEATRLKRTARRRYTRRKNRICYLOE IF SNE MAKVDDSFFHRLEESFLVEEDKKHERHPIFGNIVDEVAYHEKYPTIYHLRKKLVDSTDKADLRLIYLALAHMIKFRGHFLIEGDLNPDN SDVDKLFIQLVQTYNQLFEENPINASGVDAKAILSARLSKSRRLENLIAQLPGEKKNGLFGNLIALSLGLTPNFKSNFDLAEDAKLQLS KDTYDDDLDNLLAQIGDQYADLFLAAKNLSDAILLSDILRVNTEITKAPLSASMIKRYDEHHQDLTLLKALVRQQLPEKYKEIFFDQSK NGYAGY IDGGASOEEFYKF IKPILEKMDGTEELLVKLNREDLLRKORTFDNGS IPHOIHLGELHAILRROEDFYPFLKDNREKIEKILT FRIPYYVGPLARGNSRFAWMTRKSEETITPWNFEEVVDKGASAOSF IERMTNFDKNLPNEKVLPKHSLLYEYFTVYNELTKVKYVTEGM RKPAFLSGEQKKAIVDLLFKTNRKVTVKQLKEDYFKKIECFDSVEISGVEDRFNASLGTYHDLLKIIKDKDFLDNEENEDILEDIVLTL TLFEDREMIEERLKTYAHLFDDKVMKOLKRRRYTGWGRLSRKLINGIRDKOSGKTILDFLKSDGFANRNFMOLIHDDSLTFKEDIOKAO VSGQGDSLHEHIANLAGSPAIKKGILQTVKVVDELVKVMGRHKPENIVIEMARENQTTQKGQKNSRERMKRIEEGIKELGSQILKEHPV ENTQLQNEKLYLYYLQNGRDMYVDQELDINRLSDYDVDH IVPQSFLKDDS I DNKVLTRSDKNRGKSDNVP SEEVVKKMKNYWRQLLNAK LITORKFDNLTKAERGGLSELDKAGF IKROLVETROITKHVAOILDSRMNTKYDENDKLIREVKVITLKSKLVSDFRKDFOFYKVREIN NYHHAHDAYLNAVVGTALIKKYPKLESEFVYGDYKVYDVRKMIAKSEQEIGKATAKYFFYSNIMNFFKTEITLANGEIRKRPLIETNGE TGEIVWDKGRDFATVRKVLSMPQVNIVKKTEVQTGGF SKES ILPKRNSDKLIARKKDWDPKKYGGFDSPTVAYSVLVVAKVEKGKSKKL KSVKELLGITIMERSSFEKNPIDFLEAKGYKEVKKDLI IKLPKYSLFELENGRKRMLASAGELQKGNELALPSKYVNFLYLASHYEKLK GSPEDNEQKQLFVEQHKHYLDE I IEQISEF SKRVILADANLDKVLSAYNKHRDKP IREQAEN I IHLFTLTNLGAPAAFKYFDTTIDRKR YTSTKEVLDATLIHQSITGLYETRIDLSQLGGD

$>$ dCas9 (D10A \& H840A)

MDKKYSIGLAIGTNSVGWAVITDEYKVPSKKFKVLGNTDRHSIKKNLIGALLFDSGETAEATRLKRTARRRYTRRKNRICYLQEIF SNE MAKVDDSFFHRLEESFLVEEDKKHERHPIFGNIVDEVAYHEKYPTIYHLRKKLVDSTDKADLRLIYLALAHMIKFRGHFLIEGDLNPDN SDVDKLF IQLVQTYNQLFEENPINASGVDAKAILSARLSKSRRLENLIAQLPGEKKNGLFGNLIALSLGLTPNFKSNFDLAEDAKLQLS KDTYDDDLDNLLAOIGDOYADLFLAAKNLSDAILLSDILRVNTEITKAPLSASMI KRYDEHHODLTLLKALVROQLPEKYKEIFFDOSK NGYAGY IDGGASOEEFYKF I KPILEKMDGTEELLVKLNREDLLRKORTFDNGS IPHOIHLGELHAILRROEDFYPFLKDNREKIEKILT FRIPYYVGPLARGNSRFAWMTRKSEETITPWNFEEVVDKGASAQSF IERMTNFDKNLPNEKVLPKHSLLYEYFTVYNELTKVKYVTEGM RKPAFLSGEQKKAIVDLLFKTNRKVTVKQLKEDYFKKIECFDSVEISGVEDRFNASLGTYHDLLKIIKDKDFLDNEENEDILEDIVLTL TLFEDREMIEERLKTYAHLFDDKVMKQLKRRRYTGWGRLSRKLINGIRDKOSGKTILDFLKSDGF ANRNFMQLIHDDSLTFKEDIQKAQ VSGQGDSLHEHIANLAGSPAIKKGILQTVKVVDELVKVMGRHKPENIVIEMARENQTTQKGQKNSRERMKRIEEGIKELGSQILKEHPV ENTQLQNEKLYLYYLQNGRDMYVDQELDINRLSDYDVDAIVPQSFLKDDS IDNKVLTRSDKNRGKSDNVPSEEVVKKMKNYWRQLLNAK LITQRKFDNLTKAERGGLSELDKAGF IKRQLVETRQITKHVAQILDSRMNTKYDENDKLIREVKVITLKSKLVSDFRKDFQFYKVREIN NYHHAHDAYLNAVVGTALIKKYPKLESEFVYGDYKVYDVRKMIAKSEOE I GKATAKYFFYSNIMNFFKTEITLANGE IRKRPLIETNGE TGEIVWDKGRDFATVRKVLSMPQVNIVKKTEVQTGGF SKESILPKRNSDKLIARKKDWDPKKYGGFDSPTVAYSVLVVAKVEKGKSKKL KSVKELLGITIMERSSFEKNPIDFLEAKGYKEVKKDLI IKLPKYSLFELENGRKRMLASAGELQKGNELALPSKYVNFLYLASHYEKLK GSPEDNEQKQLFVEQHKHYLDEI IEQISEF SKRVILADANLDKVLSAYNKHRDKP IREQAENI IHLFTLTNLGAPAAFKYFDTTIDRKR YTSTKEVLDATLIHQSITGLYETRIDLSQLGGD 
$>$ Cas9-HF (N497A, R661A, Q695A, \& Q926A)

MDKKYSIGLDIGTNSVGWAVITDEYKVPSKKFKVLGNTDRHSIKKNLIGALLFDSGETAEATRLKRTARRRYTRRKNRICYLQEIFSNE MAKVDDSFFHRLEESFLVEEDKKHERHPIFGNIVDEVAYHEKYPTIYHLRKKLVDSTDKADLRLIYLALAHMIKFRGHFLIEGDLNPDN SDVDKLF IQLVQTYNQLFEENP INASGVDAKAILSARLSKSRRLENLIAQLPGEKKNGLFGNLIALSLGLTPNFKSNFDLAEDAKLQLS KDTYDDDLDNLLAQIGDQYADLFLAAKNLSDAILLSDILRVNTEITKAPLSASMIKRYDEHHQDLTLLKALVRQQLPEKYKEIFFDQSK NGYAGY IDGGASQEEFYKF IKP ILEKMDGTEELLVKLNREDLLRKQRTFDNGS IPHQI HLGELHAILRRQEDFYPFLKDNREKIEKILT FRIPYYVGPLARGNSRFAWMTRKSEETITPWNFEEVVDKGASAQSF IERMTAFDKNLPNEKVLPKHSLLYEYFTVYNELTKVKYVTEGM RKPAFLSGEQKKAIVDLLFKTNRKVTVKQLKEDYFKKIECFDSVEISGVEDRFNASLGTYHDLLKI IKDKDFLDNEENEDILEDIVLTL TLFEDREMIEERLKTYAHLFDDKVMKQLKRRRYTGWGALSRKLINGIRDKQSGKTILDFLKSDGFANRNFMALIHDDSLTFKEDIQKAQ VSGQGDSLHEH IANLAGSPAIKKGILQTVKVVDELVKVMGRHKPENIVIEMARENQTTQKGQKNSRERMKRIEEGIKELGSQILKEHPV ENTOLONEKLYLYYLONGRDMYVDOELDINRLSDYDVDH IVPOSFLKDDS IDNKVLTRSDKNRGKSDNVPSEEVVKKMKNYWROLLNAK LITQRKFDNLTKAERGGLSELDKAGF IKRQLVETRAITKHVAQILDSRMNTKYDENDKLIREVKVITLKSKLVSDFRKDFQFYKVRE IN NYHHAHDAYLNAVVGTALIKKYPKLESEFVYGDYKVYDVRKMIAKSEQEIGKATAKYFFYSNIMNFFKTEITLANGEIRKRPLIETNGE TGE IVWDKGRDFATVRKVLSMPQVNIVKKTEVQTGGF SKESILPKRNSDKLIARKKDWDPKKYGGFDSPTVAYSVLVVAKVEKGKSKKL KSVKELLGITIMERSSFEKNPIDFLEAKGYKEVKKDLIIKLPKYSLFELENGRKRMLASAGELQKGNELALPSKYVNFLYLASHYEKLK GSPEDNEQKQLFVEQHKHYLDEI IEQISEFSKRVILADANLDKVLSAYNKHRDKPIREQAENI IHLFTLTNLGAPAAFKYFDTTIDRKR YTSTKEVLDATLIHQSITGLYETRIDLSQLGGD

$>x \operatorname{Cas} 9(3.7)-B E 3$ (A262T, R324L, S409I, E480K, E543D, M694I, E1219V)

MDKKYSIGLDIGTNSVGWAVITDEYKVPSKKFKVLGNTDRHSIKKNLIGALLFDSGETAEATRLKRTARRRYTRRKNRICYLQEIFSNE MAKVDDSFFHRLEESFLVEEDKKHERHP IFGNIVDEVAYHEKYPTIYHLRKKLVDSTDKADLRLIYLALAHMIKFRGHFLIEGDLNPDN SDVDKLFIQLVQTYNQLFEENP INASGVDAKAILSARLSKSRRLENLIAQLPGEKKNGLFGNLIALSLGLTPNFKSNFDLAEDTKLQLS KDTYDDDLDNLLAOIGDOYADLFLAAKNLSDAILLSDILRVNTE ITKAPLSASMIKLYDEHHODLTLLKALVROOLPEKYKEIFFDOSK NGYAGY IDGGASQEEFYKF IKP ILEKMDGTEELLVKLNREDLLRKQRTFDNGI IPHQIHLGELHAILRRQEDFYPFLKDNREKIEKILT FRIPYYVGPLARGNSRFAWMTRKSEETITPWNFEKVVDKGASAQSF IERMTNFDKNLPNEKVLPKHSLLYEYFTVYNELTKVKYVTEGM RKPAFLSGDQKKAIVDLLFKTNRKVTVKQLKEDYFKKIECFDSVEISGVEDRFNASLGTYHDLLKI IKDKDFLDNEENEDILEDIVLTL TLFEDREMIEERLKTYAHLFDDKVMKQLKRRRYTGWGRLSRKLINGIRDKQSGKTILDFLKSDGF ANRNF IQLI HDDSLTFKEDIQKAQ VSGOGDSLHEH IANLAGSPAIKKGILOTVKVVDELVKVMGRHKPENIVIEMARENOTTOKGOKNSRERMKRIEEGIKELGSOILKEHPV ENTQLQNEKLYLYYLQNGRDMYVDQELDINRLSDYDVDH IVPQSFLKDDS IDNKVLTRSDKNRGKSDNVPSEEVVKKMKNYWRQLLNAK LITQRKFDNLTKAERGGLSELDKAGF IKRQLVETRQITKHVAQILDSRMNTKYDENDKLIREVKVITLKSKLVSDFRKDFQFYKVRE IN NYHHAHDAYLNAVVGTALIKKYPKLESEFVYGDYKVYDVRKMIAKSEQEIGKATAKYFFYSNIMNFFKTEITLANGEIRKRPLIETNGE TGEIVWDKGRDFATVRKVLSMPQVNIVKKTEVQTGGF SKESILPKRNSDKLIARKKDWDPKKYGGFDSPTVAYSVLVVAKVEKGKSKKL KSVKELLGITIMERSSFEKNPIDFLEAKGYKEVKKDLI IKLPKYSLFELENGRKRMLASAGVLQKGNELALPSKYVNFLYLASHYEKLK GSPEDNEQKQLFVEQHKHYLDE I IEQISEF SKRVILADANLDKVLSAYNKHRDKP IREQAENI I HLFTLTNLGAPAAFKYFDTTIDRKR YTSTKEVLDATLIHQSITGLYETRIDLSQLGGD

Supplemental File C5-2.

$>$ Trp_Promoter_Bacteria_Ecoli

ACATCATAACGGTTCTGGCAAATATTCTGAAATGAGCTGTTGACAATTAATCATCGAACTAGTTAACTAGTACGC

>PTS Promoter Archaea Hmediterranei

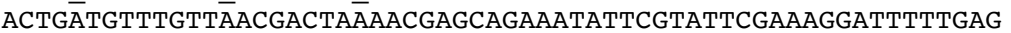

$>$ ADH 1 Promoter Fungi Scerevisiae

AtccttttgttgttĒcoggtgtacaatatggacttcctctttctggcaaccaaacccatacatcgggattcctataataccttcgtt ggtctccctaacatgtaggtggcggaggggagatatacaatagaacagataccagacaagacataatgggctaaacaagactacaccaa ttacactgcctcattgatggtggtacataacgaactaatactgtagccctagacttgatagccatcatcatatcgaagtttcactaccc tttttccatttgccatctattgaagtaataataggcgcatgcaacttcttttctttttttttcttttctctctcccccgttgttgtctc accatatccgcaatgacaaaaaatgatggaagacactaaaggaaaaattaacgacaaagacagcaccaacagatgtcgttgttccag agctgatgaggggtatctcgaagcacacgaaacttttccttccttcattcacgcacactactctctaatgagcaacggtatacggcct tccttccagttacttgaatttgaaataaaaaaagtttgctgtcttgctatcaagtataaatagacctgcaattattaatcttttgttt cctcgtcattgttctcgttcccttcttccttgtttcttttctgcacaatattcaagctataccaagcatacaatcaact

$>$ Ubi_Promoter_Plant_Zmays

ctgcāgtgcagcḡ̄gaccç̄gtcgtgcccctctctagagataatgagcattgcatgtctaagttataaaaattaccacatattttttt tgtcacacttgtttgaagtgcagtttatctatctttatacatatatttaaactttactctacgaataatataatctatagtactacaat aatatcagtgttttagagaatcatataaatgaacagttagacatggtctaaaggacaattgagtattttgacaacaggactctacagtt ttatctttttagtgtgcatgtgttctcctttttttttgcaaatagcttcacctatataatacttcatccattttattagtacatccatt tagggtttagggttaatggttttatagactaattttttagtacatctattttattctattttagcctctaaattaagaaactaaaa ctctattttagttttttatttaataatttagatataaaatagaataaaataaagtgactaaaattaaraaataccctttaagaaat taaaaaactaaggaaacatttttcttgtttcgagtagataatgccagcctgttaaacgccgtcgacgagtctaacggacaccaaccag cgaaccagcagcgtcgcgtcgggccaagcgaagcagacggcacggcatctctgtcgctgcctctggacccctctcgagagttccgctcc accgttggacttgctccgctgtcggcatccagaaattgcgtggcggagcggcagacgtgagccggcacggcaggcggcctcctcctcct ctcacggcacggcagctacgggggattcctttcccaccgctccttcgctttcccttcctcgcccgccgtaataaatagacaccccctcc acaccctctttccccaacctcgtgttgttcggagcgcacacacacacaaccagatctcccccaatccacccgtcggcacctccgcttc aaggtacgccgctcgtcctccccccccccccctctctaccttctctagatcggcgttccggtccatggttagggcccggtagttctact tctgttcatgtttgtgttagatccgtgtttgtgttagatccgtgctgctagcgttcgtacacggatgcgacctgtacgtcagacacgtt ctgattgctaacttgccagtgtttctctttggggaatcctgggatggctctagccgttccgcagacgggatcgatttcatgattttttt tgtttcgttgcatagggtttggtttgccctttcctttatttcaatatatgccgtgcacttgtttgtcgggtcatcttttcatgctttt ttttgtcttggttgtgatgatgtggtctggttgggcggtcgttctagatcggagtagaattctgtttcaaactacctggtggatttatt aatttggatctgtatgtgtgtgccatacatattcatagttacgaattgaagatgatggatggaaatatcgatctaggataggtataca tgttgatgcgggttttactgatgcatatacagagatgctttttgttcgcttggttgtgatgatgtggtgtggttgggcggtcgttcatt cgttctagatcggagtagaatactgtttcaaactacctggtgtatttattaattttggaactgtatgtgtgtgtcatacatcttcatag 
ttacgagtttaagatggatggaaatatcgatctaggataggtatacatgttgatgtgggtttactgatgcatatacatgatggcatat gcagcatctattcatatgctctaaccttgagtacctatctattataataaacaagtatgtttataattatttgatcttgatatactt ggatgatggcatatgcagcagctatatgtggattttttagccctgccttcatacgctatttatttgcttggtactgtttcttttgtcg atgctcaccctgttgtttggtgttacttctgcag

$>$ CMV_Promoter_Animal_Mmusculus

cgttācataacttācggtaaātggcccgcctggctgaccgcccaacgacccccgcccattgacgtcaataatgacgtatgttcccatag taacgccaatagggactttccattgacgtcaatgggtggagtatttacggtaaactgcccacttggcagtacatcaagtgtatcatatg ccaagtacgccccctattgacgtcaatgacggtaaatggcccgcctggcattatgcccagtacatgaccttatgggactttcctacttg gcagtacatctacgtattagtcatcgctattaccatggtgatgcggttttggcagtacatcaatgggcgtggatagcggtttgactcac ggggatttccaagtctccaccccattgacgtcaatgggagtttgttttggcaccaaaatcaacgggactttccaaaatgtcgtaacaac tccgccceattgacgcaaatgggcggtaggcgtgtacggtgggaggtctatataagcagagct

\section{Supplemental File C5-3.}

>J23119_Promoter Bacteria Ecoli

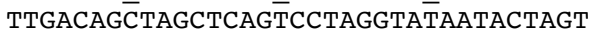

>PTS Promoter Archaea Hmediterranei

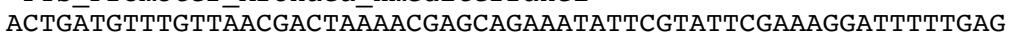

$>$ H1_Promoter_Eukaryotes_Hsapiens

GAACGCTGACGTCATCAACCCGCTCCAAGGAATCGCGGGCCCAGTGTCACTAGGCGGGAACACCCAGCGCGCGTGCGCCCTGGCAGGAA GATGGCTGTGAGGGACAGGGGAGTGGCGCCCTGCAATATTTGCATGTCGCTATGTGTTCTGGGAAATCACCATAAACGTGAAATGTCTT TGGATTTGGGAATCGTATAAGAACTGTATGAGACCAC

>U3_Promoter_Eukaryotes_osativa

AAGGAATCTTTA $\bar{A}$ ACATACGAACĀGATCACTTAAAGTTCTTCTGAAGCAACTTAAAGTTATCAGGCATGCATGGATCTTGGAGGAATCA GATGTGCAGTCAGGGACCATAGCACAAGACAGGCGTCTTCTACTGGTGCTACCAGCAAATGCTGGAAGCCGGGAACACTGGGTACGTCG GAAACCACGTGATGTGAAGAAGTAAGATAAACTGTAGGAGAAAAGCATTTCGTAGTGGGCCATGAAGCCTTTCAGGACATGTATTGCAG TATGGGCCGGCCCATTACGCAATTGGACGACAACAAAGACTAGTATTAGTACCACCTCGGCTATCCACATAGATCAAAGCTGATTTAAA AGAGTTGTGCAGATGATCCGTGGCA

>U6_Promoter_Eukaryotes_Hsapiens

AAGGTCGGGCAGGAAGAGGGCCTATTTCCCATGATTCCTTCATATTTGCATATACGATACAAGGCTGTTAGAGAGATAATTAGAATTAA TTTGACTGTAAACACAAAGATATTAGTACAAAATACGTGACGTAGAAAGTAATAATTTCTTGGGTAGTTTGCAGTTTTAAAATTATGTT TTAAAATGGACTATCATATGCTTACCGTAACTTGAAAGTATTTCGATTTCTTGGCTTTATATATCTTGTGGAAAGGACG 


\section{Primers:}

\begin{tabular}{|c|c|c|c|c|c|}
\hline Name & Sequence5 ' $->3$ ' & TM & $\begin{array}{l}\text { Lengt } \\
\mathrm{h}\end{array}$ & $\begin{array}{l}\text { Date } \\
\text { ordered }\end{array}$ & $\begin{array}{l}\text { Order } \\
\text { Ref }\end{array}$ \\
\hline MVLG_02245FW & GCAGAATTCATGACCTCACAAGTGCGAATGCAAGTCGAG & 72.1 & 39 & $31-0 c t-16$ & 1163909 \\
\hline MVLG_02245RV & GCGGCCGCGGCACTGGTTGTCGGCAGGTGCGCTAAG & 80.6 & 36 & $31-0 c t-16$ & 1163909 \\
\hline $\begin{array}{l}\text { MvSd- } \\
\text { IT02g04324FW }\end{array}$ & GCAGAATTCATGAAGCTGTCCACCTTGATCCTCACCCTT & 72.1 & 39 & $31-$ Oct-16 & 1163909 \\
\hline $\begin{array}{l}\text { MvSd- } \\
\text { IT02g04324RV }\end{array}$ & GCGGCCGCCGCCACGGCAATGCTGCTGCCGACAAGA & 80.6 & 36 & $31-0 c t-16$ & 1163909 \\
\hline MvSp880g00831FW & GCAGAATTCATGGTGTCCAAGCTGCTGGGCGCACTGGAC & 76.3 & 39 & $31-0 c t-16$ & 1163909 \\
\hline MvSp880g00831RV & GCGGCCGCTGCGAGGGCTCTTGACAAAGGAAAGAAC & 76.1 & 36 & $31-0 c t-16$ & 1163909 \\
\hline MvSp880g16237FW & GCAGAATTCATGTTCGTCTTGCTGCTCACCACCCCAGAT & 73.1 & 39 & $31-0 c t-16$ & 1163909 \\
\hline MvSp880g16237FW & GCGGCCGCTGCCAGGAGACAACCAACGCGCAATCT & 78.3 & 35 & $31-0 c t-16$ & 1163909 \\
\hline MVLG_05398F & GCAGAATTCATGCGCACCCCTTCCCTCGCGTTCGTCTTGCTT & 76.4 & 42 & 12-Dec-16 & 1181690 \\
\hline MVLG_05398R & GCGGCCGCAGCGCTGACTGCAGACGTTAGACCAAGCAAG & 64.1 & 39 & $12-$ Dec-16 & 1181690 \\
\hline MvSd01662FW & GCAGAATTCATGAGATTGCTCTTTGCTATCACCTTT & 67.0 & 36 & 23-Jan-17 & 1196597 \\
\hline MvSd01662RV & GCGGCCGCCGCATGGATCATACACACCGCGAGGCTA & 78.3 & 36 & 23-Jan-17 & 1196597 \\
\hline MvSd02874FW & GCAGAATTCATGAAGCTCCTCGCGATTGCCGTCGCC & 74.9 & 36 & 23-Jan-17 & 1196597 \\
\hline MvSd02874RV & GCGGCCGCCAGCTTGACTTGCGGCGACCCTCATGGC & 80.6 & 36 & 23-Jan-17 & 1196597 \\
\hline MvSd07159FW & GCAGAATTCATGCTTTTCCCCATCGTGTGCTTCACG & 71.5 & 36 & $23-J a n-17$ & 1196597 \\
\hline MvSd07159RV & GCGGCCGCCCCAGGTCAGGAATGTTTCAAGCAAGAG & 76.1 & 36 & $23-\mathrm{Jan}-17$ & 1196597 \\
\hline MvSp01648FW & GCAGAATTCATGCGCTTCTCCATGCTCATCCCCGTT & 72.6 & 36 & $27-F e b-17$ & 1212819 \\
\hline MvSp01648RV & GCGGCCGCCGCCGATGACGGTAGCGATGAGCGAGGC & 81.8 & 36 & $27-F e b-17$ & 1212819 \\
\hline MvSp10116FW & GCAGAATTCATGCTCCCCAGGTTATCTCGCCATCTG & 72.6 & 36 & $27-\mathrm{Feb}-17$ & 1212819 \\
\hline MvSp10116RV & GCGGCCGCCAGCAAGGCTAGGTCCTGGTGCAGAACA & 78.3 & 36 & $27-F e b-17$ & 1212819 \\
\hline MVLG_02245RVNew & GCGGCCGCCGGCACTGGTTGTCGGCAGGTGCGCTAA & 80.6 & 36 & $27-F e b-17$ & 1212819 \\
\hline MvSdG10114RVNew & GCGGCCGCCCGCCACGGCAATGCTGCTGCCGACAAG & 81.8 & 36 & $27-\mathrm{Feb}-17$ & 1212819 \\
\hline MvSp-G10007RVNew & GCGGCCGCCTGCGAGGGCTCTTGACAAAGGAAAGAA & 76.1 & 36 & $27-F e b-17$ & 1212819 \\
\hline MvSp-G15012RVNew & GCGGCCGCCTGCCAGCGAGACAACCAACGCGCAATC & 79.5 & 36 & $27-F e b-17$ & 1212819 \\
\hline tRNATrYGTAFW & GCAAAGCTTCCAATTCCTGTTCCCCCTGATTGG & 70.9 & 33 & $27-F e b-17$ & 1212819 \\
\hline tRNATrYGTARV & TCCCGCCACGCGGGATCGAACCGCGGACCCTCA & 79.6 & 33 & $27-F e b-17$ & 1212819 \\
\hline MVLG5582TarFw & $\begin{array}{l}\text { TTCGATCCCGCGTGGCGGGAGTGATTTGACGAGCCACGAGTTTTAGAGC } \\
\text { TAGAAATAGC }\end{array}$ & 78.0 & 59 & $27-F e b-17$ & 1212819 \\
\hline MVLG5582TarRV & $\begin{array}{l}\text { TTCGATCCCGCGTGGCGGGAGTGATTTGACGAGCCACGAGTTTTAGAGCT } \\
\text { AGAAATAGC }\end{array}$ & 78.0 & 59 & $27-F e b-17$ & 1212819 \\
\hline gRNAScaffoldF & $\begin{array}{l}\text { GCTATTTCTAGCTCTAAAACTCGTGGCTCGTCAAATCACTCCCGCCACGC } \\
\text { GGGATCGAA }\end{array}$ & 59.2 & 59 & $27-F e b-17$ & 1212819 \\
\hline
\end{tabular}




\begin{tabular}{|c|c|c|c|c|c|}
\hline gRNAScaffoldR & AGCACCGACTCGGTGCCACTTTTTCAAGTT & 68.7 & 30 & $27-\mathrm{Feb}-17$ & 1212819 \\
\hline PolIIITermF & AGTGGCACCGAGTCGGTGCTAAAAACTAGACCCAGCTTTCTTGTAGGTACC & 76.0 & 51 & $27-$ Feb-17 & 1212819 \\
\hline PolIIITermR & GGTACCTACAAGAAAGCTGGGTCTAGTTTTTAGCACCGACTCGGTGCCACT & 76.0 & 51 & $27-\mathrm{Feb}-17$ & 1212819 \\
\hline MVLG2523PromF & GCAGGTACCGTGCAACTGTGAGCCCCGACTCCG & 77.1 & 33 & $27-\mathrm{Feb}-17$ & 1212819 \\
\hline MVLG2523PromR & GCAGGATCCCTACGTCATGCACTGACGATGGGA & 73.4 & 33 & $27-\mathrm{Feb}-17$ & 1212819 \\
\hline CRISPRSCrnF & GCTCTACCGATGCCTTACCA & 62.4 & 20 & $24-A p r-17$ & 1240208 \\
\hline CRISPRScrnR & GCTGCGAAACTCCTTCACTC & 62.4 & 20 & $24-$ Apr-17 & 1240208 \\
\hline Cas9ScrF & СTCCACCATTCTCTCGGTCT & 62.4 & 20 & 12-May-17 & 1249612 \\
\hline Cas9ScrR & ATTGGGCGATGAGGTTTTC & 58.0 & 19 & 12-May-17 & 1249612 \\
\hline TarScrF & CAGCACCTAGCGTCGTTTTC & 62.4 & 20 & 12-May-17 & 1249612 \\
\hline TarScrR & ССTTCGTGGCTCTCGTATTT & 60.4 & 20 & 12-May-17 & 1249612 \\
\hline RnosScrF & CCGAGGACAACGAACAAAAG & 60.4 & 20 & 12-May-17 & 1249612 \\
\hline TnosScrR & GTAAAACGACGGCCAGTGAA & 60.4 & 20 & 12-May-17 & 1249612 \\
\hline 5585 Seq 50 bpUpE $1 F$ & GCAACACTCCGCCGCGGCGGCACTGCAGCTGCT & 79.6 & 33 & 21-Jul-17 & 1282410 \\
\hline $5585 \mathrm{Seq} 950 \mathrm{inR}$ & TCGTACGGGCACGAGATCTCAGCATCGGCAACC & 74.6 & 33 & 21-Jul-17 & 1282410 \\
\hline $5585 \mathrm{Seq} 950 \mathrm{inF}$ & GGTTGCCGATGCTGAGATCTCGTGCCCGTACGA & 74.6 & 33 & 21-Jul-17 & 1282410 \\
\hline $5585 \mathrm{Seq} 1.95 \mathrm{KbinR}$ & TAGGAACCAAAATCGGGGACGTTGATGCGTTTG & 69.6 & 33 & 21-Jul-17 & 1282410 \\
\hline Cas 9 Seq 50 bpupF & ACGACAAAGTGATGAAGCAGCTGAAGCGTAGGC & 70.9 & 33 & 21-Jul-17 & 1282410 \\
\hline Cas9Seq50bpUpR & TCTCTAACTTTATAGAACTGAAAATCTTTGCGG & 63.4 & 33 & 21-Jul-17 & 1282410 \\
\hline MV5585E2F & $\begin{array}{l}\text { TTCGATCCCGCGTGGCGGGACACCGACACTACGAACTGTGTTTTAGAGCT } \\
\text { AGAAATAGC }\end{array}$ & 78.0 & 59 & $26-J u l-17$ & 1286090 \\
\hline MV5585E2R & $\begin{array}{l}\text { GCTATTTCTAGCTCTAAAACACAGTTCGTAGTGTCGGTGTCCCGCCACGC } \\
\text { GGGATCGAA }\end{array}$ & 78.0 & 59 & $26-\mathrm{Jul}-17$ & 1286090 \\
\hline MV5585E4F & $\begin{array}{l}\text { TTCGATCCCGCGTGGCGGGAGCTTCCCGCTACTTCAAGAGTTTTAGAGCT } \\
\text { AGAAATAGC }\end{array}$ & 78.0 & 59 & 10 -Aug-17 & 1294116 \\
\hline MV5585E4R & $\begin{array}{l}\text { GCTATTTCTAGCTCTAAAACTCTTGAAGTAGCGGGAAGCTCCCGCCACGC } \\
\text { GGGATCGAA }\end{array}$ & 78.0 & 59 & 10 -Aug-17 & 1294116 \\
\hline $\mathrm{xCmlFW}$ & GCACCACATCTGTCTTGGGGCGATGGCGATCAGTAT & 73.8 & 36 & 10 -Aug-17 & 1294116 \\
\hline BsrGIRV & GCATGTACACTATTCCTTTGCCCTCGGACGAGTGCT & 72.6 & 36 & $10-$ Aug-17 & 1294116 \\
\hline $\operatorname{MvSd} 04324 \mathrm{R}$ & GGATCCTTAGAATCCCACGGGAAAAATAGTGGT & 68.4 & 33 & 27-Sep-17 & 1316106 \\
\hline MvSd07159F & GAATTCGAGAAGGTCCCGTCGCTCGATCGATGT & 72.1 & 33 & $27-$ Sep-17 & 1316106 \\
\hline MvSd07159R & GGATCCTTACGGACTTCTGCGTCGTCCTCGGGC & 75.8 & 33 & $27-$ Sep-17 & 1316106 \\
\hline MvSp00831F & GAATTCGACCCACCTGATTTGCGTCGCTTGACA & 70.9 & 33 & $27-$ Sep-17 & 1316106 \\
\hline MvSp00831R & GGATCCTCATAGGATTACTGCACCTAAAGCCAA & 68.4 & 33 & $27-$ Sep-17 & 1316106 \\
\hline MvSp01648F & GAATTCGGGGTCGTTCCCAAGGAGGCTCCTGTT & 73.4 & 33 & $27-$ Sep-17 & 1316106 \\
\hline MvSp01648R & GGATCCTCAGAGCTTGACGTTGTCG & 68.4 & 25 & $27-$ Sep-17 & 1316106 \\
\hline MvSp10116FW & GAATTCCGGCGGTCCGAGTCACATGCACTTGTC & 73.4 & 33 & $27-$ Sep-17 & 1316106 \\
\hline MvSp10116R & GGATCCTCACCTCGAAGCTCCCGTCCACAGCAT & 74.6 & 33 & $27-$ Sep-17 & 1316106 \\
\hline MvSp16237F & GAATTCTCCGAGCCTCGCACCGAAGGACACTGT & 73.4 & 33 & $27-$ Sep-17 & 1316106 \\
\hline MvSp16237R & GGATCCACATTGGGATCACGAGCATGGTCGTGC & 73.4 & 33 & $27-$ Sep-17 & 1316106 \\
\hline BsrGIFW & GCATGTACAGGCGATGGCGATCAGTATCACCTTGCT & 72.6 & 36 & $27-$ Sep-17 & 1316106 \\
\hline MVLG_02245F & GAATTCGCACCGCTGGCTTCGGAGCAAATCTCG & 73.4 & 33 & $15-$ Sep-17 & 1310443 \\
\hline MVLG_02245R & GGATCCGGGTGATGCTGTGGTAGTATGATAGCG & 72.1 & 33 & $15-$ Sep-17 & 1310443 \\
\hline
\end{tabular}




\begin{tabular}{|c|c|c|c|c|c|}
\hline MVLG_05398F & GAATTCATTGCCGTTGGTGAACCCA & 72.1 & 25 & $15-$ Sep-17 & 1310443 \\
\hline MVLG_05398R & GGATCCGGCGCTGGGATCCGGCGTSAGTGGGAAT & 77.1 & 34 & 15-Sep-17 & 1310443 \\
\hline MvSd01662F & GAATTCCCTTCCCGCCGAAGACCACAGCGACAC & 74.6 & 33 & 15-Sep-17 & 1310443 \\
\hline MvSd01662R & GGATCCTCATACATGGCCGTGTCCCTGGTAGGG & 74.6 & 33 & 15-Sep-17 & 1310443 \\
\hline MvSd02874F & GAATTCACACCAGACCCTATGTCTGGTAGCAGC & 70.9 & 33 & $15-$ Sep-17 & 1310443 \\
\hline MvSd02874R & GGATCCTCAGCAATGAAACTTGGAATGACAACC & 68.4 & 33 & 15-Sep-17 & 1310443 \\
\hline MvSd04324F & GAATTCGCACCGGCACCCCTAATCGACGGCGGC & 77.1 & 33 & $15-$ Sep-17 & 1310443 \\
\hline MvSd04324R & GGATCCTTAGAATCCCACGGGAAAAATAGTGGT & 68.4 & 33 & $15-$ Sep-17 & 1310443 \\
\hline MvSd662FW & GCAGTTAATTAAATGAGATTGCTCTTTGCTATCACC & 65.8 & 36 & $10-0 c t-17$ & 1321828 \\
\hline MvSd662RV & GCAGATATCGATCTCAGGGTCCTGCCCTATCTCAAT & 71.5 & 36 & $10-0 c t-17$ & 1321828 \\
\hline MvSd874FW & GCAGTTAATTAAATGAAGCTCCTCGCGATTGCCGTC & 70.4 & 36 & $10-0 c t-17$ & 1321828 \\
\hline MvSd874RV & GCAGATATCGATCAAGACGTGATCACGTGCGACACT & 71.5 & 36 & $10-0 c t-17$ & 1321828 \\
\hline MvSd324FW & GCAGTTAATTAAATGAAGCTGTCCACCTTGATCCTC & 68.1 & 36 & $10-0 c t-17$ & 1321828 \\
\hline MvSd324RV & GCAGATATCGCAAAAACGGTAATGCCGAATTAGACT & 68.1 & 36 & $10-0 c t-17$ & 1321828 \\
\hline MvSd159FW & GCAGTTAATTAAATGCTTTTCCCCATCGTGTGCTTC & 68.1 & 36 & $10-0 c t-17$ & 1321828 \\
\hline MvSd159RV & GCAGATATCGCTTTCCACTGCCTCGCATCTCCACCT & 73.8 & 36 & $10-$ Oct-17 & 1321828 \\
\hline MvSp16237YSTFW & $\begin{array}{l}\text { GCAGAATTCATGTTCGTCTTGCTGCTCACCACCCCAGATTGCGCGTTGGT } \\
\text { TGTCTCGCTG }\end{array}$ & 79.1 & 60 & $17-0 c t-17$ & 1325248 \\
\hline MvSp16237YSTRV & $\begin{array}{l}\text { GCAGCGGCCGCTGCCAGCGAGACAACCAACGCGCAATCTGGGGTGGTGAG } \\
\text { CAGCAAGACG }\end{array}$ & 83.9 & 60 & $17-0 c t-17$ & 1325248 \\
\hline MvSd01662F & TGCATGACGTAGATGAGATTGCTCTTTGCTATCACC & 69.2 & 36 & 01-Nov-17 & 1332464 \\
\hline MvSd02874F & TGCATGACGTAGATGAAGCTCCTCGCGATTGCCGTC & 73.8 & 36 & $01-$ Nov-17 & 1332464 \\
\hline MvSd0 $43245 \mathrm{~F}$ & TGCATGACGTAGATGAAGCTGTCCACCTTGATCCTC & 71.5 & 36 & $01-$ Nov-17 & 1332464 \\
\hline MvSd07159F & TGCATGACGTAGATGCTTTTCCCCAATCGTGTGCTC & 71.5 & 36 & $01-$ Nov-17 & 1332464 \\
\hline MvSp00831F & TGCATGACGTAGATGGTGTCCAAGCTGCTGGGCGCA & 74.9 & 36 & 01-Nov-17 & 1332464 \\
\hline MvSp01648F & TGCATGACGTAGATGCGCTTCTCCATGCTCATCCCC & 73.8 & 36 & $01-$ Nov-17 & 1332464 \\
\hline MvSp10116F & TGCATGACGTAGATGCTCCCCAGGTTATCTCGCCAT & 72.6 & 36 & 01-Nov-17 & 1332464 \\
\hline MVLG2523PromF & GCATTAATTAAGTGCAACTGTGAGCCCCGACTCCGT & 71.5 & 36 & $01-$ Nov-17 & 1332464 \\
\hline MVLG2523PromR & CTACGTCATGCACTGACGATGGGATCGAGA & 70.1 & 30 & $01-$ Nov-17 & 1332464 \\
\hline MvSp831R & GCAGATATCACGCGGTCGAACCCGAACCCGAAGCTA & 58.3 & 36 & 11-Dec-17 & 1349588 \\
\hline MvSp1648R & GCAGATATCGCGGCACCCACTACCGGGTCTCTCTCT & 61.1 & 36 & 11-Dec-17 & 1349588 \\
\hline MvSp10116R & GCAGATATCGCAGGTGGGGGACGGAGAAATTGCAGC & 58.3 & 36 & $11-$ Dec-17 & 1349588 \\
\hline MVLG_05703Lf & GACGGGAGACAAACAAGCAT & 60.4 & 20 & 15-Jan-18 & 1361290 \\
\hline MVLG_05703Rt & СTTCCTCGGGTGAATAGACG & 62.4 & 20 & 15-Jan-18 & 1361290 \\
\hline MVLG_00961Lf & TGGTTTGACTTTGGCTGTTG & 58.4 & 20 & 15-Jan-18 & 1361290 \\
\hline MVLG_00961Rt & CATTTGGTGGAAGATGAGCA & 58.4 & 20 & $15-J a n-18$ & 1361290 \\
\hline MVLG_06117Lf & ATGCCAAGGTTGTGCTCTTT & 58.4 & 20 & $15-J a n-18$ & 1361290 \\
\hline MVLG_06117Rt & АCTCATTCCAGGCCATGTTG & 60.4 & 20 & $15-J a n-18$ & 1361290 \\
\hline MVLG_03216Lf & GGCGAAACTCCTTTTGATGA & 58.4 & 20 & $15-J a n-18$ & 1361290 \\
\hline MVLG_03216Rt & TCGTGGTGGAAGTTGTGGTA & 60.4 & 20 & $15-J a n-18$ & 1361290 \\
\hline MVLG_05589Lf & АТСССТGСТСТСТTСTTCAА & 58.4 & 20 & $15-J a n-18$ & 1361290 \\
\hline MVLG_05589Rt & GTCGACGTATTCGAGGTGGT & 62.4 & 20 & $15-J a n-18$ & 1361290 \\
\hline
\end{tabular}




\begin{tabular}{|c|c|c|c|c|c|}
\hline Cas9/gRNAFw & TTAATTAACCAATTCCTGTTCCCCCTGATTGGCCTA & 68.1 & 36 & $11-\mathrm{Feb}-18$ & 1375008 \\
\hline Cas9/gRNARv & GATACTTCATGTTTGACAGCTTATCATCGGATCTAG & 67.0 & 36 & $11-\mathrm{Feb}-18$ & 1375008 \\
\hline sgRNAFw & GCATTAATTAACCAATTCCTGTTCCCCCTGATTGGC & 69.2 & 36 & $11-\mathrm{Feb}-18$ & 1375008 \\
\hline SgRNARv & TACTTTGTCACCTGCAGGTACAAGAAAGCTGGGTCT & 70.4 & 36 & $11-\mathrm{Feb}-18$ & 1375008 \\
\hline $5703-\mathrm{PFw}$ & TGTACCTGCAGGTGACAAAGTAGTAMAGTGGAAGCGT & 70.4 & 37 & $11-\mathrm{Feb}-18$ & 1375008 \\
\hline 5703-PRV & GCATTGTACAACCGAGGCGCGAGCTTGCTTCCGAAA & 73.8 & 36 & $11-\mathrm{Feb}-18$ & 1375008 \\
\hline $\mathrm{HF}-\mathrm{Cas} 9 \mathrm{Fw}$ & GCCTCGGTTGTACAATGCCGCCTAAGAAGAAACGCA & 72.6 & 36 & $11-\mathrm{Feb}-18$ & 1375008 \\
\hline HF-Cas9Rv & GCAGATATCTCATGTTTGACAGCTTATCATCGGATC & 68.1 & 36 & $11-\mathrm{Feb}-18$ & 1375008 \\
\hline 5703-P_ALTFW & TGTACCTGCAGGTCCTGCCAGTGCTTCCTC & 72.8 & 30 & $23-\mathrm{Feb}-18$ & 1381641 \\
\hline MVLG_p1A1-PRFw & $\begin{array}{l}\text { TTCGATCCCGCGTGGCGGGATCCGTTTCAGCAATTTACGGTTTTAGAGCT } \\
\text { AGAAATAGC }\end{array}$ & 49.2 & 59 & 01-Mar-18 & 1384908 \\
\hline MVLG_p1A1-PRRV & $\begin{array}{l}\text { GCTATTTCTAGCTCTAAAACCGTAAATTGCTGAAACGGATCCCGCCACGC } \\
\text { GGGATCGAA }\end{array}$ & 49.2 & 59 & $01-\operatorname{Mar}-18$ & 1384908 \\
\hline MvSp831Fw & GGAGAATTCGACCCACCTGATTTGCGTCGCTTGACA & 52.8 & 36 & $01-\operatorname{Mar}-18$ & 1384908 \\
\hline MvSd4324Fw & GGAGAATTCGCACCGGCACCCCTAATCGACGGCGGC & 66.7 & 36 & $01-$ Mar-18 & 1384908 \\
\hline MVLG_00933F & GCAGAATCCATGTTATCTCGTCGAAAGTGGTCC & 69.6 & 33 & 13-Mar-18 & 1391073 \\
\hline MVLG_00933R & GCAGCGGCCGCCGGCGCGAACGGGAGGCAGCGC & 84.5 & 33 & 13-Mar-18 & 1391073 \\
\hline MVLG_00934F & GCAGAATCCATGTACCAAGACGCAGCAGCGCCG & 74.6 & 33 & 13-Mar-18 & 1391073 \\
\hline MVLG_00934R & GCAGCGGCCGCCCGGCTGCACCGAATGTGTGTT & 78.3 & 33 & $13-\operatorname{Mar}-18$ & 1391073 \\
\hline MvSd1141Gg0 $04324 \mathrm{R}$ & GCAGGATCCGGAAGGATGGGGGAGGAT & 72.2 & 27 & 28-Mar-18 & 1398840 \\
\hline Cas9TrunkFw & GATCAAGAAAGGTATCCTCCAGACCGTCAA & 67.4 & 30 & $04-A p r-18$ & 1402535 \\
\hline Cas9TrunkRv & TTGACGGTCTGGAGGATACCTTTCTTGATC & 67.4 & 30 & $04-\mathrm{Apr}-18$ & 1402535 \\
\hline GibsonoriAmpFw & GCTGTCAAACATGAGATATCTCGCAGCCACCCACAGTA & 72.2 & 38 & $23-A p r-18$ & 1412131 \\
\hline GibsonoriAmpRv & ACAGGAATTGGTTAATTAAAGGGGATAACGCAGGAAAGA & 67.8 & 39 & $23-\mathrm{Apr}-18$ & 1412131 \\
\hline pMvCC9CutFw & GCAGCAGCAGCATTAATTAACCAATTCCTGTTCCCCCTGATTGGC & 73.8 & 45 & 17-May-18 & 1425299 \\
\hline pMvCC9CutRv & GCAGCAGCAGCAGATATCTCATGTTTGACAGCTTATCATCGGATC & 72.9 & 45 & 17-May-18 & 1425299 \\
\hline sgRNAFw & GCAGATATCCCAATTCCTGTTCCCCCTGATTGG & 70.9 & 33 & 25-May-18 & 1430012 \\
\hline SgRNARV & GCATCTAGATACAAGAAAGCTGGGTCTAGTTTT & 65.9 & 33 & 25-May-18 & 1430012 \\
\hline Cas $9 \mathrm{Fw}$ & GCATTAATTAAGTGCAACTGTGAGCCCCGACTC & 69.6 & 33 & 25-May-18 & 1430012 \\
\hline Cas9RvBam & GATGTCGAGTCCGATGCTGT & 62.4 & 20 & $06-\mathrm{Jul}-18$ & 1451819 \\
\hline AmpORI $\mathrm{FW}$ & TTAATTAATGCTGCTGCTGATGAGTATTCAACATTTCCG & 66.8 & 39 & $09-0 c t-18$ & 1500901 \\
\hline $5703 \mathrm{GibFw}$ & АGCTTTCTTGTACCTGCAGGTCCTGCCAGTGCTTCCTCGAAAAAACGC & 52.1 & 48 & $05-$ Sep-18 & 1483894 \\
\hline Pol III Gib Fw & $\begin{array}{l}\text { TTGAAAAAGTGGCACCGAGTCGGTGCTAAAAACTAGACCCAGCTTTCTTG } \\
\text { TACCTGCAGG }\end{array}$ & 48.3 & 60 & $05-$ Sep-18 & 1483894 \\
\hline Pol III Gib RV & $\begin{array}{l}\text { CCTGCAGGTACAAGAAAGCTGGGTCCCTGCAGGTACAAGAAAGCTGGGTC } \\
\text { АСTTTTTCAA }\end{array}$ & 48.3 & 60 & $05-$ Sep-18 & 1483894 \\
\hline Msd13216F & TTAATTAATTCCGGCGATTCGGTCCGGGCGAAAAACGT & 71.2 & 38 & 05-Dec-18 & 1529639 \\
\hline Msd13216R & GATATCGGATGGAAAGCGTGGAGAGGAGGAGAGTGG & 73.8 & 36 & $05-$ Dec-18 & 1529639 \\
\hline Msd12336F & TTAATTAAATGAACCATCGCTGTCGCACCATGGTCTGT & 69.0 & 38 & 05-Dec-18 & 1529639 \\
\hline Msd12336R & TCTAGACTGAGCCCGAACCTGAGCCCGAACCTGAAC & 74.9 & 36 & $05-$ Dec-18 & 1529639 \\
\hline Msd10910F & TTAATTAAGGCAATGTGCATCAGTCTCCACGGGGCGGA & 72.2 & 38 & $05-$ Dec-18 & 1529639 \\
\hline Msd10910R & TCTAGACTTGTCTTTTCGTGCGTACALTGCCGTCGGC & 72.6 & 37 & $05-$ Dec-18 & 1529639 \\
\hline Msd09295F & TTAATTAAGTTCGTCTCTTGGACGTAGACGGCCGAATG & 70.1 & 38 & $05-$ Dec-18 & 1529639 \\
\hline Msd09295R & TCTAGACTAGCGTGTCCACTTGAGTTTGGTCCCAGA & 71.5 & 36 & $05-$ Dec-18 & 1529639 \\
\hline
\end{tabular}




\begin{tabular}{|c|c|c|c|c|c|}
\hline Overlap-HYG-EcoRV & CGGGTACCGAGCTCGATATCTCATGTTTGACAGCTTATCATCGGATCT & 74.1 & 48 & $16-J a n-19$ & 1545605 \\
\hline 5585_up_flFw & TTACCTAAAATTTGCAACTATGAT & 54.3 & 24 & $20-\operatorname{Mar}-19$ & 1577478 \\
\hline 5585_up_flRv & ACAGTTGCACTCGGTGGGTGGGGAGCTTTT & 71.5 & 30 & $20-\operatorname{Mar}-19$ & 1577478 \\
\hline MVLG_2523_Fw & CACCCACCGAGTGCAACTGTGAGCCCCGAC & 75.6 & 30 & $20-\operatorname{Mar}-19$ & 1577478 \\
\hline MVLG_RV & TGCTCAGCATCTACGTCATGCACTGACGAT & 68.7 & 30 & $20-\operatorname{Mar}-19$ & 1577478 \\
\hline CFP_FW & CATGACGTAGATGCTGAGCAAGGGCGAGGA & 71.5 & 30 & $20-\operatorname{Mar}-19$ & 1577478 \\
\hline CFP_RV & GCGGCAACCATTACTTGTACAGCTCGTCCA & 70.1 & 30 & $20-\operatorname{Mar}-19$ & 1577478 \\
\hline dwn_Fw & GTACAAGTAATGGTTGCCGCAGCAGAAAGT & 67.4 & 30 & $20-\operatorname{Mar}-19$ & 1577478 \\
\hline dwn_Rv & ССTTGTAGTTGGGGTTGCATTTGA & 62.9 & 24 & $20-\operatorname{Mar}-19$ & 1577478 \\
\hline $5730 \mathrm{P}-\mathrm{Cas} 9 \mathrm{FW}$ & CACCCACCGATCCTGCCAGTGCTTCCTCGA & 74.2 & 30 & $20-\operatorname{Mar}-19$ & 1577478 \\
\hline $5730 \mathrm{P}-\mathrm{Cas} 9 \mathrm{Rv}$ & GCGGCAACCACTTCTTCTTCTTGGCCTGTC & 71.5 & 30 & $20-\operatorname{Mar}-19$ & 1577478 \\
\hline Tel5703PCas9Fw & CAGTGCACGAGCAATCGACCGTGCACACTAGACCCAGCTTTCTTGTAGGTA & 76.8 & 51 & $20-\operatorname{Mar}-19$ & 1577478 \\
\hline Tel5703PCas9Rv & АСАCTAGACCCAGCTTTCTTGTAGGACCCAGGGTITTCCCAGTCACG & 79.0 & 47 & $20-$ Mar-19 & 1577478 \\
\hline Alt_dwn_Rv_1 & TCTTCATCTGCACCAAGTCG & 60.4 & 20 & $16-$ Apr-19 & 1591255 \\
\hline Alt_dwn_Rv_2 & ССTTGTAGTTGGGGTTGCAT & 60.4 & 20 & $16-$ Apr-19 & 1591255 \\
\hline tRNAgRNAcasHF $1 \mathrm{~F}$ & GGTACCCCAATTCCTGTTCCCCCTGATTGG & 56.7 & 30 & $26-A p r-19$ & 1596432 \\
\hline tRNAgRNACaSHF $1 \mathrm{~F}$ & TCTAGAAAAAAAGCACCGACTCGGTGCCACT & 48.4 & 31 & $26-$ Apr-19 & 1596432 \\
\hline \multirow[t]{4}{*}{$80 \mathrm{mer} \mathrm{Fw}$} & GTACAAGTAACGATGGTTGCCGCAGCAGAAAGTTCCGCACACCAACGGAC & 62.5 & 80 & 06-May-19 & 1600822 \\
\hline & GGCCCTCGTGATTGGCGCCGGCGTCGGAGG & & & & \\
\hline & CCTCCGACGCCGGCGCCAATCACGAGGGCCGTCCGTTGGTGTGCGGAACT & 62.5 & 80 & 06-May-19 & 1600822 \\
\hline & TTCTGCTGCGGCAACCATCGTTACTTGTAC & & & & \\
\hline Dwn Flank Rv & CCTCCGACGCCGGCGCCAAT & 75.0 & 20 & $06-$ May-19 & 1600822 \\
\hline U6_ovlp Fw & GATTACGAATTCTTAATTAACGACCAGAGAGAGAGGCAGA & 68.8 & 40 & 23-Aug-19 & 1644769 \\
\hline U6_ovlp Rv & TCGTGGCTCGTCAAATCACGTTGTAGAATGGAATTTTG & 69.0 & 38 & 23-Aug-19 & 1644769 \\
\hline Tar/Scaf Fw & CAAAATTCCATTCTACAACGTGATTTGACGAGCCACGA & 69.0 & 38 & 23-Aug-19 & 1644769 \\
\hline Tar/Scaf Rv & CGGGTACCGAGCTCGATATCAAAAAAGCACCGACTCGGTG & 75.0 & 40 & 23-Aug-19 & 1644769 \\
\hline tRNA/sgRNA Fw & GGCCGTCGTTTTACTCTAGACCAATTCCTGTTCCCCCTGA & 73.9 & 40 & 30-Aug-19 & 1648403 \\
\hline tRNA/sgRNA Rv & GGCGCGCCCCTAGGAGGCCTAAAAAAGCACCGACTCGGTG & 79.0 & 40 & 30 -Aug-19 & 1648403 \\
\hline U6/sgRNA & GGCCGTCGTTTTACTCTAGATAATACGTTCGTTCCGATGT & 70.8 & 40 & 30 -Aug-19 & 1648403 \\
\hline pGA2pGB FW & TGGCCATGGAGGCCGAATTCTACCCATACGACGTACCAGAT & 74.7 & 41 & $09-0 c t-19$ & 1665031 \\
\hline pGA2pGB Rv & CGCTGCAGGTCGACGGATCCTGCACGATGCACAGTTGAAG & 77.0 & 40 & $09-0 c t-19$ & 1665031 \\
\hline $5703-\mathrm{P} F \mathrm{~F}$ & GATTACGAATTCTTAATTAAAAAAAACGCAGCAACACATT & 63.7 & 40 & $09-0 c t-19$ & 1665031 \\
\hline 5703-P Rv & TTCGCACTTGTGAGGTCATCGGCATATGTGTCGTGAAGG & 73.1 & 39 & $09-0 c t-19$ & 1665031 \\
\hline $2245 \mathrm{FW}$ & ССTTCACGACACATATGCCGATGACCTCACAAGTGCGAA & 73.1 & 39 & $09-0 c t-19$ & 1665031 \\
\hline $2245 \mathrm{RV}$ & ACCATAGAGCCGCCAGAGCCGCCCGGAACAACCCTTGTGGTGG & 80.0 & 43 & $09-0 c t-19$ & 1665031 \\
\hline EGFP FW & TTCCGGGCGGCTCTGGCGGCTCTATGGTGAGCAAGGGCGAGGA & 80.9 & 43 & $09-0 c t-19$ & 1665031 \\
\hline EGFP RV & СTCTGCAGGTCGACTCTAGAGATCTAGTAACATAGATGAC & 70.8 & 40 & $09-0 c t-19$ & 1665031 \\
\hline Cas9F & AGCGAGTGATCTTGGCTGAT & 60.4 & 20 & $30-0 c t-19$ & 1673975 \\
\hline Cas9R & CGATGCTTGTTGTAGGCAGA & 60.4 & 20 & $30-0 c t-19$ & 1673975 \\
\hline tRNAsgRNAaltF & CGAGCCACGAGTTTTAGAGC & 62.4 & 20 & $30-$ Oct-19 & 1673975 \\
\hline
\end{tabular}




\begin{tabular}{|c|c|c|c|c|c|}
\hline tRNAsgRNAaltR & CGGACTAGCCTTATTTTAACTT & 57.1 & 22 & $30-0 c t-19$ & 1673975 \\
\hline MvSl1693MvSdF & CGATTTCACGAAGCAGAACA & 58.4 & 20 & $30-0 c t-19$ & 1673975 \\
\hline MvSl1693MvSdR & GTCGAGGGGTGACGAAAGTC & 64.5 & 20 & $30-0 c t-19$ & 1673975 \\
\hline MvSd9295MvSlaltF & CCAGCATACTGTTGTGTCTGC & 62.6 & 21 & $30-0 c t-19$ & 1673975 \\
\hline MvSd9295MvSlaltR & CCAGCATACTGTTGTGTCTGC & 58.4 & 21 & $30-0 c t-19$ & 1673975 \\
\hline MVLG2245GFPF & TATATCATGGCCGACAAGCA & 58.4 & 20 & $30-$ Oct-19 & 1673975 \\
\hline MVLG2245GFPR & GTTGTGGCGGATCTTGAAGT & 60.4 & 20 & $30-0 c t-19$ & 1673975 \\
\hline MVLG5585SeqFW & СTCCGATAGCGACTCACCTC & 64.5 & 20 & $08-$ Nov-19 & 1678214 \\
\hline MVLG5585SeqRV & CTCCGATAGCGACTCACCTC & 64.5 & 20 & $08-N o v-19$ & 1678214 \\
\hline MVLG2245SeqFw & GGCACTGGTAGGTATCTTCGAT & 62.7 & 22 & 08-Nov-19 & 1678214 \\
\hline MVLG2245SeqRV & AGAGCGAGCAACAACCTCAT & 60.4 & 20 & 08-Nov-19 & 1678214 \\
\hline MvSl1693SeqFw & AGAGCGAGCAACAACCTCAT & 60.8 & 20 & 08-Nov-19 & 1678214 \\
\hline MvSl1693SeqRv & GAGATGTTCTGCTTCGTGAAATC & 61.0 & 23 & 08 -Nov-19 & 1678214 \\
\hline NewTargetRNAPFW & AGCGTCGTTITCCCGCCATA & 62.4 & 20 & 08-Nov-19 & 1678214 \\
\hline NewTargetRNAPRV & AGCGTCGTTITCCCGCCATA & 70.6 & 20 & 08-Nov-19 & 1678214 \\
\hline NewTargetScafFw & AGCGTCGTTITCCCGCCATA & 54.2 & 20 & 08-Nov-19 & 1678214 \\
\hline NewTargetScafRv & GCATGCGGCGCGCCCCTAGG & 72.7 & 20 & 08-Nov-19 & 1678214 \\
\hline MVLG2245TaroligoF & GTTCGATCCCGCGTGGCGGGACTTGCATTCGCACTTGTGGTTTTAGAGCTAGAAATAGC & 78.0 & 59 & 08-Nov-19 & 1678217 \\
\hline MVLG2245TarOligoR & GСTATTTCTAGCTCTAAAACCACAAGTGCGAATGCAAGTCCCGCCACGCG & 78.0 & 73 & 08-Nov-19 & 1678217 \\
\hline & TGCGAATGCAAGTCCCGCCACGC & & & & \\
\hline MvSl1693TarOligoF & $\begin{array}{l}\text { GTTCGATCCCGCGTGGCGGGCTGCGTTCGATCCCGCGTGGCGGGCTGCTA } \\
\text { GAAATAGC }\end{array}$ & 78.7 & 58 & 08-Nov-19 & 1678217 \\
\hline MvS11693TaroligoR & $\begin{array}{l}\text { GCTATTTCTAGCTCTAAAACAATGCGACCACAAGAGCAGCCCGCCACGCG } \\
\text { GGATCGAAC }\end{array}$ & 78.7 & 59 & 08-Nov-19 & 1678217 \\
\hline MVLG2245ScafF & АСTTGCATTCGCACTTGTGGTTTTAGAGCTAGAAATAGC & 68.9 & 39 & 08-Nov-19 & 1678217 \\
\hline MVLG2245tRNAPR & CACAAGTGCGAATGCAAGTCCCGCCACGCGGGATCGAAC & 77.3 & 39 & 08-Nov-19 & 1678217 \\
\hline U6_ovlp Fw & GGCCGTCGTTTTACTCTAGACGACCAGAGAGAGAGGCAGA & 75.0 & 40 & $20-$ Nov-19 & 1682701 \\
\hline U6_ovlp Rv & CTCGTGGCTCGTCAAATCACGTTGTAGAATGGAATTTTGA & 69.8 & 40 & 20 -Nov-19 & 1682701 \\
\hline Tar/Scaf Fw & TCAAAATTCCATTCTACAACGTGATTTGACGAGCCACGAG & 69.8 & 40 & 20 -Nov-19 & 1682701 \\
\hline Tar/Scaf Rv & GGCGCGCCCCTAGGAGGCCTAAAAAAGCACCGACTCGGTG & 79.0 & 40 & $20-$ Nov-19 & 1682701 \\
\hline MVLG5 703ExpressF & СТСААТСАССССТСGСТTC & 62.3 & 19 & 05-Dec-19 & 1688287 \\
\hline MVLG5703ExpressR & СTGTTGCCTCATTTTGTCGT & 58.4 & 20 & $05-$ Dec-19 & 1688287 \\
\hline $\mathrm{t}-\mathrm{g} \mathrm{FW}$ & GCTTTGGAAGAGCATCAGAC & 60.4 & 20 & 09-Dec-19 & 1689480 \\
\hline$t-g \mathrm{RV}$ & СTAAAACTCGTGGCTCGTCA & 60.4 & 20 & 09-Dec-19 & 1689480 \\
\hline g Fw & GTGATTTGACGAGCCACGAG & 62.4 & 20 & 09-Dec-19 & 1689480 \\
\hline g Rv & CGGACTAGCCTTATTTTAACTTGC & 61.2 & 24 & 09-Dec-19 & 1689480 \\
\hline 1693 qrt Fw & AACGGTGAAGAAGGAGCAAA & 58.4 & 20 & 09-Dec-19 & 1689480 \\
\hline 1693 qrt Rv & TCGTGAGACGCCGAAGTAAA & 60.4 & 20 & 09-Dec-19 & 1689480 \\
\hline MVLG_05589-P FW & GATTACGAATTCTTAATTAACGCCAGTGTGCTGGAATTCG & 68.8 & 40 & 12-Dec-19 & 1691181 \\
\hline MVLG_05589-P RV & TTGCGTTTCTTCTTAGGCATTGGGCGAAGAGAGGATGCGA & 72.9 & 40 & $12-$ Dec-19 & 1691181 \\
\hline Cas9-Frag Fw & TCGCATCCTCTCTTCGCCCAATGCCTAAGAAGAAACGCAA & 72.9 & 40 & $12-$ Dec-19 & 1691181 \\
\hline Cas9-Frag Rv & CTGCGTGTATAACGTCGACGTGCCGTGCGTTTGAGGCGTG & 77.0 & 40 & 12-Dec-19 & 1691181 \\
\hline
\end{tabular}




\begin{tabular}{|c|c|c|c|c|c|}
\hline MVLG5589PRV2245 & АTTCGCACTTGTGAGGTCATTGGGCGAAGAGAGGATGCGA & 73.9 & 40 & $12-\operatorname{Dec}-19$ & 1691181 \\
\hline MVLG_02245 Fw & TCGCATCCTCTCTTCGCCCAATGACCTCACAAGTGCGAAT & 73.9 & 40 & 12-Dec-19 & 1691181 \\
\hline Short-U6 Fw & tctagaCGACCAGAGAGAGAGGCAGA & 67.7 & 26 & $10-J a n-20$ & 1698963 \\
\hline Short-U6 Rv & GTTGTAGAATGGAATTTTGA & 52.2 & 20 & $10-J a n-20$ & 1698963 \\
\hline Short-U6Tar Fw & GTGATTTGACGAGCCACGAG & 62.4 & 20 & $10-J a n-20$ & 1698963 \\
\hline Short-U6Tar Rv & aggcctAAAAAAGCACCGACTCGGTG & 67.7 & 26 & $10-J a n-20$ & 1698963 \\
\hline Cas9-1 Fw & ССТСTCTCGGCACCTATCAC & 64.5 & 20 & $10-J a n-20$ & 1698963 \\
\hline Cas9-1 Rv & TCCTCGTTCTCTTCGTTGTC & 60.4 & 20 & $10-J a n-20$ & 1698963 \\
\hline Cas9-2 Fw & ССТСTCTCGGCACCTATCAC & 64.5 & 20 & $10-J a n-20$ & 1698963 \\
\hline Cas9-2 Rv & ATCCTCGTTCTCTTCGTTGTC & 60.6 & 21 & $10-J a n-20$ & 1698963 \\
\hline Cas9-3 Fw & TGCTCAСССТСАСТTTGTTC & 60.4 & 20 & $10-J a n-20$ & 1698963 \\
\hline Cas9-3 Rv & TGCTTCATCACTTTGTCGTC & 58.4 & 20 & $10-J a n-20$ & 1698963 \\
\hline $4324-\mathrm{P} F \mathrm{Fw}$ & gattacgaattcttaattaaCACGGTGCAGCATGACGAAG & 68.8 & 40 & $10-J a n-20$ & 1698963 \\
\hline 4324-P Rv & GCGACCACAAGAGCAGCATTTTCGCCCACACATCACTCAA & 73.9 & 40 & $10-J a n-20$ & 1698963 \\
\hline MvS1-1064_01693Fw & TTGAGTGATGTGTGGGCGAAAATGCTGCTCTTGTGGTCGC & 73.9 & 40 & $10-J a n-20$ & 1698963 \\
\hline MvSl-1064_01693Rv & ctctgcaggtcgactctagaTCAATTGTTCTTGATGGTAA & 69.8 & 40 & $10-J a n-20$ & 1698963 \\
\hline Whole U6 Fw & GGCCGTCGTTTTACTCTAGATAATACGTTCGTTCCGATGT & 70.8 & 40 & $20-J a n-20$ & 1702482 \\
\hline Whole U6 Rv & СTCGTGGCTCGTCAAATCACGTTGTAGAATGGAATTTTGA & 69.8 & 40 & $20-J a n-20$ & 1702482 \\
\hline WU6-sgRNA FW & TCAAAATTCCATTCTACAACGTGATTTGACGAGCCACGAG & 69.8 & 40 & $20-J a n-20$ & 1702482 \\
\hline WU6-sgRNA Rv & TGCGGCGCGCCCCTAGGAGGCCTAAAAAAGCACCGACTCG & 79 & 40 & $20-J a n-20$ & 1702482 \\
\hline$\beta$-Car-Hyg Fw & $\begin{array}{l}\text { TGATTTGACGAGCCACGATGGTTGCCGCAGCGCCAGTGTG } \\
\text { CTGGAATTCGCCCTTGCTGT }\end{array}$ & 80.5 & 60 & $28-J a n-20$ & 1706129 \\
\hline$\beta$-Car-Hyg Rv & $\begin{array}{l}\text { CCTAATCAAGAAGTCAACTATGCGCTCCCTCTATTCCTTT } \\
\text { GCCCTCGGACGAGTGCTGGG }\end{array}$ & 78.4 & 60 & $28-J a n-20$ & 1706129 \\
\hline MvSl_01693Fw60 & $\begin{array}{l}\text { GCCTCGTACCATGCTGCTCTTGTGGTCGCACGCCAGTGTG } \\
\text { CTGGAATTCCCCTTGCTGT }\end{array}$ & 81.2 & 60 & $28-J a n-20$ & 1706129 \\
\hline MvSl_01693Rv60 & $\begin{array}{l}\text { CGCTCCTCTAATTTCAATTGTTCTTGATGGCTATTCCTTT } \\
\text { GCCCTCGGACGAGTGCTGGG }\end{array}$ & 77.1 & 60 & $28-J a n-20$ & 1706129 \\
\hline$\beta-\mathrm{CarFw}$ & CCGCTCGGGTCCTCAGGCTCGGCCT & 76.1 & 25 & $28-J a n-20$ & 1706129 \\
\hline$\beta-$ CarRv & TGTACAAAGGTCTGTATGCTTCTAA & 59.7 & 25 & $28-J a n-20$ & 1706129 \\
\hline MvSl_01693Fw25 & CGTTACGCCTCTTGTCCTTTTGCTT & 64.6 & 25 & $28-J a n-20$ & 1706129 \\
\hline MvSl_01693Rv25 & СTTGTTGTGATGTATAAAAGATGTC & 58 & 25 & $28-J a n-20$ & 1706129 \\
\hline MvSl_01693 1kbFw & CGCCAACTTGGTTTCACTATGCGCC & 67.9 & 25 & $28-J a n-20$ & 1706129 \\
\hline MvSl_01693 1kbRv & GAAGGGGCGGGCAGGACAATCAAGA & 69.5 & 25 & $28-J a n-20$ & 1706129 \\
\hline 1693 1kb_OvlFw & ACCATGATTACGAATTCTTACGCCAACTTGGTTTCACTATGCGCC & 72 & 45 & $28-J a n-20$ & 1706129 \\
\hline 1693 1kb_OvlRv & ССТCTGCAGGTCGACTCTAGGAAGGGGCGGGCAGGACAATCAAGA & 78.4 & 45 & $28-J a n-20$ & 1706129 \\
\hline FixMVLG05589PFw & GATTACGAATTCTTAATTAAGTTTAAACTGAAGGCGGGAA & 65.7 & 40 & $28-J a n-20$ & 1706129 \\
\hline 2245-GFPnoSP FW & TCGCATCCTCTCTTCGCCCAGCACCGCTGGCTTCGGAGCA & 79 & 40 & $17-\mathrm{Feb}-20$ & 1715090 \\
\hline 2245-GFPnoSP RV & СTCTGCAGGTCGACTCTAGAGATCTAGTAACATAGATGAC & 70.8 & 40 & $17-\mathrm{Feb}-20$ & 1715090 \\
\hline New2245-GFPnoSP FW & TCGCATCСTСTCTTCGCCCAATGGCACCGCTGGCTTCGGA & 78 & 40 & $25-\mathrm{Feb}-20$ & 1718964 \\
\hline New2245-GFPnoSP RV & TCCGAAGCCAGCGGTGCCATTGGGCGAAGAGAGGATGCGA & 78 & 40 & $25-\mathrm{Feb}-20$ & 1718964 \\
\hline
\end{tabular}


Freezer Stocks:

Freezer Box \#1 Listed Index

\begin{tabular}{|c|c|c|}
\hline Grid & Plasmid & Cell Type \\
\hline A1 & PMV_HYG & In DH5a \\
\hline A2 & ВP 6175 3-1 & \\
\hline A3 & BP 859 C9 & In DH5a \\
\hline A4 & MvSp_01648 In pYST0 & In Yeast \\
\hline A5 & MVLG_5378 Signal Pep. In pYST0 & In Yeast \\
\hline A6 & MvSd_04324-P In TOPO & In Yeast \\
\hline A7 & MvSd_02874-P In TOPO & In Yeast \\
\hline A8 & MvSd_07159-P In TOPO & In Yeast \\
\hline A9 & MvSd_01662 In pYST0 & In Yeast \\
\hline B1 & pMv_育yg & In DH5a \\
\hline B2 & Lu53 & Lu53 \\
\hline B3 & DH5a & DH5a \\
\hline B4 & MvSp_01648 In pYST0 & In Yeast \\
\hline B5 & MVLG_02245 In pYST0 In Frame & In SEY \\
\hline B6 & MvSp A2 Stock & MvSp A2 \\
\hline B7 & MvSp A2 Stock & MvSp A2 \\
\hline B8 & MvSp A2 Stock & MvSp A2 \\
\hline B9 & MvSp A2 Stock & MvSp A2 \\
\hline C1 & EHA105 & EHA105 \\
\hline $\mathrm{C2}$ & BsrGI-Hyg-BsrGI In TOPO & In DH5a \\
\hline C3 & Bacilis subtilis & B. subtilis \\
\hline C4 & - & - \\
\hline C5 & MvSp_16237 nIn pYST0 In Frame & In SEY \\
\hline C6 & MvSp A1 Stock & MvSp A1 \\
\hline C7 & MvSp A1 Stock & MvSp A1 \\
\hline C8 & MvSp A1 Stock & MvSp A1 \\
\hline C9 & MvSp A1 Stock & MvSp A1 \\
\hline D1 & pMS8 2-1 & In DH5a \\
\hline D2 & pMS8 2-2 & In DH5a \\
\hline D3 & Proto3 & In DH5a \\
\hline D4 & MvSd_07159 In pYST0 In Frame & In SEY \\
\hline D5 & MvSd_01662 In pYST0 In Frame & In SEY \\
\hline D6 & MvSd A2 Stock & MvSd A2 \\
\hline D7 & MvSd A2 Stock & MvSd A2 \\
\hline D8 & MvSd A2 Stock & MvSd A2 \\
\hline D9 & MvSd A2 Stock & MvSd A2 \\
\hline E1 & pMS10 2-1 & In DH5a \\
\hline E2 & pMS10 2-2 & In DH5a \\
\hline E3 & Proto2 E1 target + Mv Promoter for Cas9 & In DH5a \\
\hline
\end{tabular}




\begin{tabular}{|c|c|c|}
\hline E4 & MvSp_01648 In pYST0 & In DH5a \\
\hline E5 & MvSp_01648 In pYST0 & In DH5a \\
\hline E6 & MvSd A1 Stock & MvSd A1 \\
\hline E7 & MvSd A1 Stock & MvSd A1 \\
\hline E8 & MvSd A1 Stock & MvSd A1 \\
\hline E9 & MvSd A1 Stock & MvSd A1 \\
\hline F1 & pMs 73 A & In DH5a \\
\hline F2 & pMs 73 B & In DH5a \\
\hline $\mathbf{F 3}$ & Proto 1 pMS8+Frag & In DH5a \\
\hline $\mathbf{F 4}$ & MVLG_02245 In pYST0 In Frame & In SEY \\
\hline $\mathbf{F 5}$ & MVLG_05398 In pYST0 In Frame & In SEY \\
\hline F6 & MvSI p1A2 Stock & MvSI p1A2 \\
\hline F7 & MvSI p1A2 Stock & MvSI p1A2 \\
\hline F8 & MvSl p1A2 Stock & MvSI p1A2 \\
\hline F9 & MvSI p1A2 Stock & MvSI p1A2 \\
\hline G1 & Cas9 HF & In DH5a \\
\hline G2 & CRISPR Frag In TOPO & In DH5a \\
\hline G3 & MVLG_02523 Promoter In TOPO & In DH5a \\
\hline G4 & MvSp_00831 In pYST0 In Frame & In SEY \\
\hline G5 & MvSd_02874 In pYST0 In Frame & In SEY \\
\hline G6 & MvSI p1A1 Stock & MvSI p1A1 \\
\hline G7 & MvSI p1A1 Stock & MvSI p1A1 \\
\hline G8 & MvSl p1A1 Stock & MvSI p1A1 \\
\hline G9 & MvSI p1A1 Stock & MvSI p1A1 \\
\hline H1 & A2 STC & \\
\hline H2 & A2 STC & \\
\hline H3 & A2 STC & \\
\hline H4 & A2 STC & \\
\hline H5 & A2 STC & \\
\hline H6 & A2 STC & \\
\hline H7 & A2 STC & \\
\hline H8 & A2 STC & \\
\hline H9 & A2 STC & \\
\hline I1 & A2 STC & \\
\hline 12 & A2 STC & \\
\hline I3 & A2 STC & \\
\hline I4 & A2 STC & \\
\hline 15 & A2 STC & \\
\hline I6 & A2 STC & \\
\hline 17 & A2 STC & \\
\hline I8 & A2 STC & \\
\hline I9 & A2 STC & \\
\hline
\end{tabular}


Freezer Box \#2 Listed Index

\begin{tabular}{|c|c|c|}
\hline Grid & Plasmid & Cell Type \\
\hline$\overline{\mathrm{A1}}$ & Y2H 2245 In TOPO & In DH5a \\
\hline A2 & MvSd_09295 In TOPO & In DH5a \\
\hline A3 & MvSd_10910 In TOPO & In DH5a \\
\hline A4 & MvSd_12336 In TOPO & In DH5a \\
\hline A5 & MvSd_13216 In TOPO & In DH5a \\
\hline A6 & Y2H Control pGADT7 T7 & In Y187 \\
\hline A7 & Y2H Control pGBKT7 53 & in $Y 187$ \\
\hline A8 & Nelson's Signal Peptide 5122 & In Yeast \\
\hline A9 & Nelson's pYST0 Vector & In Yeast \\
\hline B1 & pGADT7 MVLG_02245 & In DH5a \\
\hline B2 & MvSd_09295 In pMvHyg & In DH5a \\
\hline B3 & pGBKT7_Q1 & In DH5a \\
\hline B4 & pGBKT7_Q3 & In DH5a \\
\hline B5 & pGBKT7_Q5 & In DH5a \\
\hline B6 & pGBKT7_Q6 & In DH5a \\
\hline B7 & pGADT7_Q1 & In Y187 \\
\hline B8 & pGADT7_Q3 & In Y187 \\
\hline B9 & pGBKT7_2245 & In AH109 \\
\hline C1 & pGBKT7 MVLG_02245 & In DH5a \\
\hline $\mathrm{C2}$ & MvSd_09295 In pMvHyg & In DH5a \\
\hline C3 & pGB_Q1 & in $\mathrm{AH} 109$ \\
\hline $\mathrm{C4}$ & pGB_Q3 & in $\mathrm{AH} 109$ \\
\hline C5 & pGB_Q5 & in $\mathrm{AH} 109$ \\
\hline C6 & pGB_Q6 & in $\mathrm{AH} 109$ \\
\hline C7 & pGADT77Q5 & In Y187 \\
\hline C8 & pGADT7_Q6 & In Y187 \\
\hline C9 & pGB_2245 X pGA_Q6 & Yeast Diploids \\
\hline D1 & - & - \\
\hline D2 & MvSd_09295 In pMvHyg & In DH5a \\
\hline D3 & pGB_Q1 X pGA_2245 & Yeast Diploids \\
\hline D4 & pGB_Q3 X pGA_2245 & Yeast Diploids \\
\hline D5 & pGB_Q5 X pGA_2245 & Yeast Diploids \\
\hline D6 & pGB_Q6 X pGA_2245 & Yeast Diploids \\
\hline D7 & pGB_2245 X pGĀ_Q1 & Yeast Diploids \\
\hline D8 & pGB_2245 X pGA_Q3 & Yeast Diploids \\
\hline D9 & pGB_2245 X pGA_Q5 & Yeast Diploids \\
\hline E1 & Infected S. latifolia Prey Library & In Y187 \\
\hline E2 & Infected S. latifolia Prey Library & In Y187 \\
\hline E3 & Infected S. latifolia Prey Library & In Y187 \\
\hline E4 & pMvHyg_tRNA_CRISPR & In DH5a \\
\hline E5 & T\#140 CRISPR-Trans & MvSI p1A1 \\
\hline E6 & pMvHyg_MvSI_01693 & MvSd A1 \\
\hline E7 & pMvHyg_MvSI_01693 & MvSd A2 \\
\hline E8 & pMvHyg_No-SP-MVLG_02245-GFP & MvSl p1A1 \\
\hline E9 & pMvHyg_No-SP-MVLG_02245-GFP & MvSl p1A2 \\
\hline F1 & pMvHyg_Cas9 & In DH5a \\
\hline F2 & Infected $\bar{S}$. latifolia Prey Library & In Y187 \\
\hline F3 & pMvHyg_CRISPR & In DH5a \\
\hline F4 & pMvHyg_sgRNA T & In DH5a \\
\hline F5 & pMvHyg_sgRNA & In EHA105 \\
\hline F6 & pBG_53 Control & In AH109 \\
\hline F7 & - & - \\
\hline F8 & - & - \\
\hline F9 & pMvHyg_CRISPR Transformant \#2 & in p1A1 \\
\hline
\end{tabular}




\begin{tabular}{|c|c|c|}
\hline G1 & pCFP & In DH5a \\
\hline G2 & Burkholderia Cenocpacia J2315 & B. Cenocpacia \\
\hline G3 & pMvHyg_6175 & In DH5a \\
\hline G4 & pMvHyg_sgRNA B & In DH5a \\
\hline G5 & Rosetta $E$. coli & Rosetta $E$. coli \\
\hline G6 & pGA_T7 Control & In Y187 \\
\hline G7 & - & - \\
\hline G8 & - & - \\
\hline G9 & pMvHyg_CRISPR Transformant \#3 & in $\mathbf{p} 1 \mathrm{~A} 1$ \\
\hline H1 & pET_Cas9 in ADD GENE Bacteria & ADD GENE Bacteria \\
\hline H2 & Star One Shot Cells & BL21 (DE3) \\
\hline H3 & pET_Cas9 & BL21 (DE3) \\
\hline H4 & pMvHyg_sgRNA In TOPO & In DH5 $\alpha$ \\
\hline H5 & pET_Cas9 & In BL21 (DE3) \\
\hline H6 & pGBKT7 & In DH5a \\
\hline H7 & - & - \\
\hline H8 & - & - \\
\hline H9 & pMvHyg_CRISPR Transformant \#4 & in p1A1 \\
\hline I1 & MVLG_SAD F1 Gen Strain \#1 & MvSl p1A1 \\
\hline $\mathbf{I 2}$ & MVLG_SAD F1 Gen Strain \#2 & MvSl p1A2 \\
\hline $\mathbf{I 3}$ & MVLG_SAD F1 Gen Strain \#1 & MvSl p1A2 \\
\hline I4 & MVLG_SAD F1 Gen Strain \#2 & MvSI p1A2 \\
\hline I5 & pMvHyg_SAD & In DH5a \\
\hline I6 & pGADT7 & In DH5a \\
\hline I7 & pMvHyg_MvSl-01693 & In DH5 $\alpha$ \\
\hline I8 & pMvHyg_MvSl-01693 & In EHA105 \\
\hline 19 & pMvHyg_CRISPR Transformant \#5 & in $\mathbf{p} 1 \mathrm{~A} 1$ \\
\hline
\end{tabular}




\section{Protocols:}

\section{ATMT of Microbotryum}

\section{Mating the Cells}

- $\quad$ Prepare $1 \mathrm{LB}+\mathrm{Kan}+$ Spec plate, $1 \mathrm{YPD}-10 \%+$ Amp plate, $1 \mathrm{IM}$ plate, $5 \mathrm{~mL}$ of IM broth , 5 $\mathrm{mL}$ YPD $-10 \%$ broth, and 10 YPD $-10 \%+$ Hyg + Cef plates per transformation.

[See next page for media prep]

- Streak out Agrobacterium (EHA105) with your construct on LB + Kan + Spec and grow at $28^{\circ} \mathrm{C}$ for 2 days.

*(EHA105 uses a binary plasmid system and must be plated on Kan \& Spec)

- Streak out Microbotryum on YPD - 10\% + Amp and grow at $28^{\circ} \mathrm{C}$ for 2 days.

- Scrape a loopful of cells into $1 \mathrm{~mL}$ of IM broth (less is more).

- Vortex until completely suspended.

- Dilute 10X in IM broth and check OD.

- Dilute original stocks to $1 \mathrm{E} 8$ cells each

○ D Microbotryum $\mathrm{OD}_{600} 1=3.4 \mathrm{E} 7$ cells $/ \mathrm{mL}$

- Agrobacterium $\mathrm{OD}_{600} 1=8 \mathrm{E} 8$ cells $/ \mathrm{mL}$

- Mix the following volumes of $1 \mathrm{E} 8$ cells $/ \mathrm{mL}$ to achieve the listed ratios of total cells:

\begin{tabular}{|c|c|c|c|}
\hline $\begin{array}{c}\text { Resulting Ratio of } \\
\text { Total Cells }\end{array}$ & $\begin{array}{c}\text { Volume of } \\
\text { Agrobacterium } \\
\text { cells }(\boldsymbol{\mu L})\end{array}$ & $\begin{array}{c}\text { Volume of } \\
\text { Microbotryum cells } \\
(\boldsymbol{\mu L})\end{array}$ & $\begin{array}{c}\text { Volume of } \\
\text { IM } \\
(\boldsymbol{\mu L})\end{array}$ \\
\hline 1E7 M: 1 E7Agro & 100 & 100 & 0 \\
\hline 1E6 M: 1 E7Agro & 100 & 10 & 90 \\
\hline
\end{tabular}

- $\quad$ Spot $200 \mu \mathrm{L}$ of each mixture onto a different sides of an IM plate *(Make sure surface is level so that the mixtures don't run and merge)

- Let plates sit at room temperature, lid up, for 2-3 days.

\section{Selecting for Transformants}

- $\quad$ Scrape each mass of cells into $2 \mathrm{~mL}$ of YPD - $10 \%$ broth resuspend.

- $\quad$ Spread $200 \mu \mathrm{L}$ of suspension onto a YPD 10\% + Hyg + Cef plate.

- Allow 2-3 weeks of growth for transformed Microbotryum colonies to appear 


\section{Media Prep}

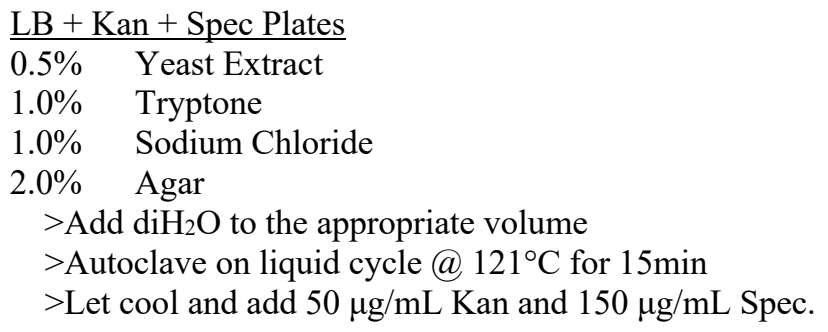

IM Broth $(50 \mathrm{~mL})$

$20 \mathrm{~mL}$ 2.5X MM Salts

$45 \mathrm{mg}$ Dextrose

$250 \mu \mathrm{L}$ Glycerol $>$ Add $27 \mathrm{~mL} \mathrm{diH} \mathrm{m}_{2} \mathrm{O}$

$>$ Autoclave on liquid cycle @ $121^{\circ} \mathrm{C}$ for $15 \mathrm{~min}$

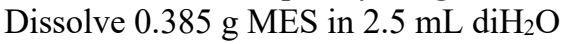

Dissolve $0.95 \mathrm{mg}$ Acetosyringone in $12.5 \mu \mathrm{L}$ DMSO

*(It is better to make this along with the mixture of the IM Plates and just $12.6 \mathrm{~mL}$ of the mixture) $>$ Mix MES and Acetosyringone

$>$ Let cool and add MES/Aceto mixture to media

\begin{tabular}{ll}
$2.5 \mathrm{X}$ MM Salts $(1 \mathrm{~L})$ \\
\hline $\mathrm{KH}_{2} \mathrm{PO}_{4}$ & $3.625 \mathrm{~g}$ \\
$\mathrm{~K}_{2} \mathrm{HPO}_{4}$ & $5.125 \mathrm{~g}$ \\
$\mathrm{NaCl}$ & $0.375 \mathrm{~g}$ \\
$\mathrm{MgSO}_{4} .7 \mathrm{H}_{2} \mathrm{O}$ & $1.250 \mathrm{~g}$ \\
$\mathrm{CaCl}_{2} .2 \mathrm{H}_{2} \mathrm{O}$ & $0.165 \mathrm{~g}$ \\
$\mathrm{FeSO}_{4} .7 \mathrm{H}_{2} \mathrm{O}$ & $6.2 \mathrm{mg}$ \\
$\left(\mathrm{NH}_{4}\right)_{2} \mathrm{SO}_{4}$ & $1.250 \mathrm{~g}$
\end{tabular}




\section{CRISPR Kit Transformation of Microbotryum Protoplasts}

\section{Preparing Cas9 Duplex}

- Resuspend the crRNA and tracrRNA to $100 \mu \mathrm{M}$ stock concentrations in the provided NucleaseFree Duplex Buffer.

\begin{tabular}{|c|c|}
\hline Normalized Amount Delivered (nmol) & Duplex Buffer Resuspension Volume $(\boldsymbol{\mu L})$ \\
\hline 2 & 20 \\
\hline 5 & 50 \\
\hline 10 & 100 \\
\hline 20 & 200 \\
\hline 100 & 1000 \\
\hline
\end{tabular}

- Store resuspended RNA oligos at $-20^{\circ} \mathrm{C}$

- For complete gene deletion, use two gRNAs, one that targets a PAM site at the 5' end and one that targets a PAM site at the 3 ' end.

- For each gRNA, prepare a $33 \mu \mathrm{M}$ RNA duplex solution by mixing the crRNA and corresponding tracrRNA in equimolar concentration.

\begin{tabular}{lc} 
Component & Amount $(\boldsymbol{\mu L})$ \\
$100 \mu \mathrm{M}$ Alt-R CRISPR-Cas9 crRNA & 5 \\
$100 \mu$ M Alt-R CRISPR-Cas9 tracrRNA & 5 \\
Nuclease-Free Duplex Buffer & $\underline{5}$ \\
\multicolumn{1}{c}{ Total Volume } & 15
\end{tabular}

- Heat at $95^{\circ} \mathrm{C}$ for 5 minutes and then allow to cool to room temperature

Note: The crNRA:tracrRNA guide complex can be used for 3 months with no loss in activity when stored at $-20^{\circ} \mathrm{C}$.

- Before use, thoroughly mix the stock Alt-R S.p. Cas9 Nuclease 3NLS by inverting the tube. Quick spin. Dilute Cas 9 10X to a final concentration of $1 \mu \mathrm{g} / \mu \mathrm{L}$ (recommend using $1 \mu \mathrm{L}$ of Cas 9 and 9 $\mu \mathrm{L}$ of Cas9 working buffer, this will allow for 6 reactions).

- Combine the following:

\begin{tabular}{|c|c|}
\hline Component & Amount $(\mu \mathrm{L})$ \\
\hline $33 \mu \mathrm{M}$ RNA duplex solution 1 (gRNA 1) & 1.5 \\
\hline $33 \mu \mathrm{M}$ RNA duplex solution 2 (gRNA 2) & 1.5 \\
\hline Cas9 nuclease $(1 \mu \mathrm{g} / \mu \mathrm{L})$ & \\
\hline Cas9 Working Buffer & $\underline{22}$ \\
\hline Total Volume & 26.5 \\
\hline
\end{tabular}

- Incubate at room temperature for 5 min to allow the assembly of the RNP complexes.

Cas9 Working Buffer:

20 mM HEPES (pH 7.5)

$150 \mathrm{mM} \mathrm{KCl}$
$800 \mu \mathrm{L}$

$20 \mu \mathrm{L} \mathrm{1M} \mathrm{HEPES} \mathrm{(pH} \mathrm{7.5)}$

$150 \mu \mathrm{L} 1 \mathrm{M} \mathrm{KCl}$

$>630 \mu \mathrm{L}$ Nuclease-Free Water 


\section{Transformation}

- $\quad$ Retrieve protoplasts form $-80^{\circ} \mathrm{C}$ storage and thaw on ice.

- $\quad$ Pipette $100 \mu \mathrm{L}$ of STC solution into a round bottom $15 \mathrm{~mL}$ snap cap tube and place on ice.

- $\quad$ Add $7 \mu \mathrm{g}$ of purified repair template (plasmid, cosmid, or linear construct). Do not add more than $20 \mu \mathrm{L}$ of DNA.

- $\quad$ Add $26.5 \mu \mathrm{L}$ of the RNP complex

- $\quad$ Add $200 \mu \mathrm{L}$ of protoplasts using wide-bore tips, carefully and slowly pipetting the mixture to ensure even suspension without destroying the cells.

- Add $50 \mu \mathrm{L}$ of $30 \%$ PEG solution. Mix carefully by gentle swirling or slowly pipetting up and down with wide-bore tips and incubate on ice for $50 \mathrm{~min}$.

- Add $2 \mathrm{~mL}$ of $30 \%$ PEG solution, mixing carefully via gentle inversion. Incubate at room temp. for 20 minutes. Cells should clump together during this incubation, which can be verified microscopically. (During this step, add top agar to your regeneration plates)

- Add $2 \mathrm{~mL}$ of STC and mix thoroughly via gentle inversion of the tube.

- $\quad$ Pipette $500 \mu \mathrm{L}$ of the transformation mixture to the top agar of the selection plates. Tilt plates to help spread the mixture evenly, but do not spread. (This will make up to 8 plates)

- Incubate at $\sim 25^{\circ} \mathrm{C}$ until colonies form.

- Re-streak colonies onto YPD-10\% $150 \mu \mathrm{g} / \mathrm{mL}$ Hyg plates.

\section{Materials}

STC Solution:

1.2 M Sorbitol

$50 \mathrm{mM} \mathrm{CaCl}_{2} .2 \mathrm{H}_{2} \mathrm{O}$

10 mM Tris-HCL (pH 8.0)

30\% PEG Solution:

30\% PEG 8000

$50 \mathrm{mM} \mathrm{CaCl}_{2}$

10 mM Tris- $\mathrm{HCl}$ (pH 8.0)
$10 \mathrm{~mL}$

$2.18 \mathrm{~g}$

$500 \mu \mathrm{L} 1 \mathrm{M}$ solution

$100 \mu \mathrm{L} 1 \mathrm{M}$ solution

\author{
$10 \mathrm{~mL}$ \\ $3 \mathrm{~g}$ \\ $500 \mu \mathrm{L} 1 \mathrm{M}$ solution \\ $100 \mu \mathrm{L} 1 \mathrm{M}$ solution
}

Top Agar Selection Plates:

>Make $100 \mathrm{~mL}$ of bottom and $100 \mathrm{~mL}$ of top agar for 10 plates:

YPD-10\% Plates:

$1 \mathrm{~g}$ Yeast Extract

2 g Peptone

20 g Dextrose (Separate Flask)

2 g Agar

$>$ Autoclave

$>$ Add $300 \mu \mathrm{g} / \mathrm{mL}$ Hyg-B to bottom agar

$>$ Make bottom agar the same day you plan to do the transformations (storage lowers efficiency).

$>$ Plate $10 \mathrm{~mL}$ of bottom agar, let solidify. During 20 minute incubation period, add $10 \mathrm{~mL}$ top agar. 


\section{Designing Primers for CRISPR Kit}

\section{Selecting Target}

Targets for the Cas 9 endonuclease should be 20 bp in length preceding an NGG PAM site. These targets should be unique in the genome, at least with regards to the last $10 \mathrm{bp}$ before the PAM site. GGG PAM sites should also be avoided if possible.

Ex:

$$
\begin{array}{llll} 
& \text { Target } & & \text { PAM } \\
5^{\prime} & \text { GTGTGCGGAACTTTCTGCTG } & \text { 3' } & \text { CGG }
\end{array}
$$

*Note: it does not matter in which direction the target is located as Cas9 cuts across both strands.

To increase likelihood of knockout, two targets should be designed ( 1 at the 5' end of the target gene and the other at the 3' end of the target gene). By using two targets, the target is excised completely from the genome.

When using the CRISPR Kit, a sgRNA is generated using hydrogen binding of two components, the crRNAs (the targets you design) and the tracrRNA (the scaffold that holds the crRNA to Cas9). These are generated automatically when you input your $20 \mathrm{bp}$ target sequence into the program provided by the company to order your crRNAs. Because the tracrRNA and Cas 9 are the same regardless of target, these are supplied separately and do not require adjustment.

\section{Repair Template}

In addition to your dual target sequences, a selectable marker with $60 \mathrm{bp}$ of overlapping sequence to the genome immediately upstream for the 5' target and downstream of the 3' target should be generated. This can be accomplished by designing a $60 \mathrm{bp}$ oligonucleotide primer for your insert with $20 \mathrm{bp}$ for priming the sequence and $40 \mathrm{bp}$ of overlap. While $40 \mathrm{bp}$ of overlap may suffice for insertion in some systems, $60 \mathrm{bp}$ is optimal. Furthermore, amplifying inserts from genomic DNA with such a large primer can be difficult. To increase your length of overlap, and to isolate inserts from genomic DNA, nested PCR is recommended.

To perform a nested PCR resulting in an amplified insert with the $60 \mathrm{bp}$ corresponding overlap sequence, a series of 3 primers can be used ( 2 sets if the aforementioned 60 bp oligonucleotide successfully amplifies your target.

1) An initial 20 bp Forward and Reverse primer without overhangs can be used to amplify the target from genomic DNA. The resulting fragment should be run on a gel and gel purified for use as the template in step 2.

2) A second set of $60 \mathrm{bp}$ oligonucleotides with $20 \mathrm{bp}$ of primer to the fragment amplified in step 1 can be used to amplify the fragment, adding $40 \mathrm{bp}$ of complementary sequence corresponding to the upstream of the 5 ' target and downstream of the 3 ' target.

3) A third set of $40 \mathrm{bp}$ oligonucleotides with $20 \mathrm{bp}$ of primer to the fragment amplified in step 2 can be used to amplify the fragment, adding an additional $20 \mathrm{bp}$ of complementary sequence. This will yield an insert with $60 \mathrm{bp}$ of complementary

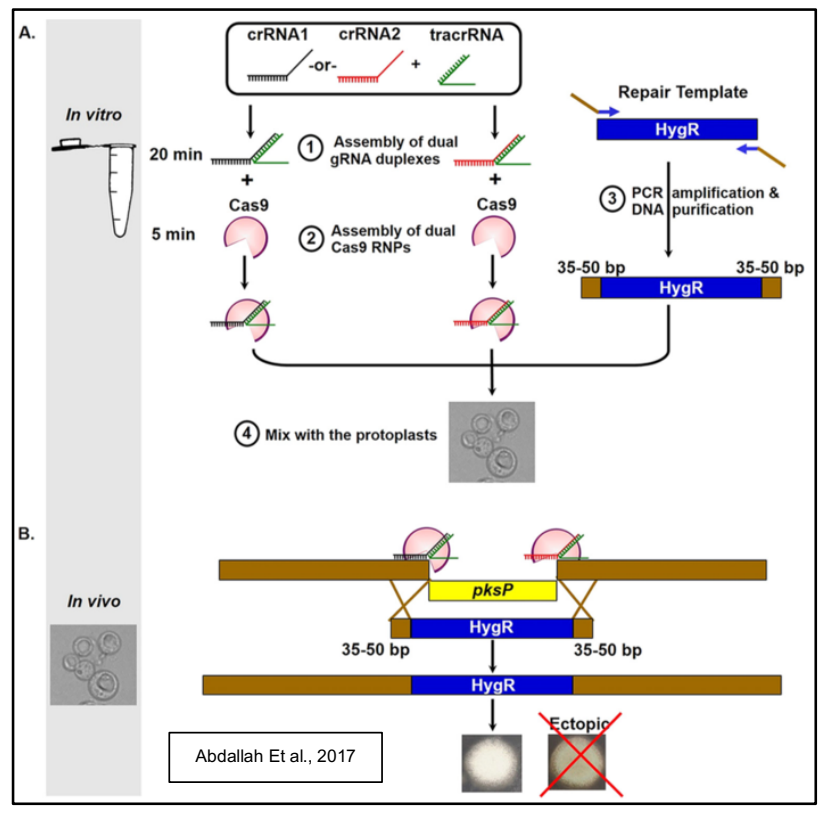
sequence to the boundaries of the excised target for the Cas9 kit.

For the pilot study for use of the CRISPR Kit in Microbotryum lychnidis-dioicae, the 5589-Hyg resistance cassette from pMvHyg was amplified as the selectable insert for knockout of MVLG_05585. 
*Note: because the flanking regions are different depending on your targets, the insert with complementary sequence must be generated from new primers every time a new target is used.

\section{How to Order the Materials}

Once you have designed your target sequences, the crRNAs can be ordered from the following site: https://www.idtdna.com/site/order/oligoentry/index/crispr

In addition to your custom crRNAs, you will also need to order tracrRNA and the Cas9 endonuclease *Note: the company sells multiple variants of the Cas9 endonuclease, including the single mutant nickase (Cas9n) and the double mutant Cas9 for CRISPRi (dCas9).

Primers for amplification of the selectable insert should be ordered through Eurofins. 


\section{Infecting Silene}

\section{Inoculating Seeds}

- Grow A1 and A2 Microbotryum mating types separate on YPD-10\% for 2 days.

- Inoculate $1 \mathrm{~mL}$ of water with a loop of cells separately from each mating type.

- Check optical density of mixtures and adjust to $1.0 \mathrm{~A}_{600 \mathrm{~nm}}$.

- Plate 40-50 seeds equal distance from each other on large 1.8\% water agar plate.

- Mix equal volumes of each mating type mixture and spot $\sim 50 \mu \mathrm{L}$ onto each seed.

- Place plates face up in $12 \mathrm{C}^{\circ}$ chamber $\left(4 \mathrm{C}^{\circ}\right.$ will work if needed) for $48 \mathrm{~h}$.

- Move plates to growth chambers and spot the seeds with more water every 2-3 days when the water from the previous spotting is gone. Make sure plates are wrapped to prevent drying out.

\section{Transplanting Sprouts}

- Once the seeds begin to sprout and form the cotyledon, cut away the agar around the root and transplant into egg-shell crate using propagation mix as the soil. It is helpful to lightly pack the soil and "prime" it by adding a little water to the top to help it draw up water from the tray. Toothpicks can also be used to help prop sprouts up until they become established.

- Cover the tray with a lid to help keep humidity up.

- Water seeds from the bottom up using a tray tap water.

\section{Transplanting Young Plants}

- Once the sprouts are at the 4-8 leaves stage, transplant them into small tray pots using Sta-Green potting mix. When transplanting from an eggshell crate, first fill the small pots with soil and lightly pack. Prime the soil with water and let sit until there is no standing water (5-10 minutes). Poking holes can help this process along as packing the soil can make it slower to soak up the water. Once the soil is primed, create a plug for the entire eggshell crate soil plug and transplant the young plants.

- Continue to use the lid on the trays until the plants are too tall for it. It may then be useful to prop up taller plants with wooden rods.

- If plants have not bolted after 2 months of growth, begin watering them by alternating between Bloom-Booster fertilizer and tap water. 


\title{
Making Competent DH5a Cells
}

- Make $50 \mathrm{~mL}$ LB liquid Media in a $200 \mathrm{~mL}$ flask and $5 \mathrm{~mL}$ LB liquid Media in a $50 \mathrm{~mL}$ flask.

\author{
LB Liquid Media \\ $0.5 \% \quad$ Yeast Extract \\ $1.0 \% \quad$ Tryptone \\ $1.0 \% \quad$ Sodium Chloride \\ $>$ Add $\mathrm{diH}_{2} \mathrm{O}$ to the appropriate volume \\ $>$ Autoclave on liquid cycle @ $121^{\circ} \mathrm{C}$ for $15 \mathrm{~min}$
}

- T-streak fresh DH5 $\alpha$ cells onto an LB plate and incubate at $37^{\circ} \mathrm{C}$ for $24 \mathrm{~h}$.

- Pick a single colony of DH5 $\alpha$ and inoculate the flask of $5 \mathrm{~mL} \mathrm{LB}$.

- Shake the inoculum at $37^{\circ} \mathrm{C}$ overnight ( 12-16h).

- Use a $1000 \mu \mathrm{L}$ pipette to transfer $500 \mu \mathrm{L}$ of the overnight culture to the $50 \mathrm{~mL}$ LB flask.

- Shake the new inoculum at $37^{\circ} \mathrm{C}$, taking absorbance readings every half hour, starting after $2 \mathrm{~h}$ of shaking, until the $\mathrm{OD}_{600 \mathrm{~nm}}$ is between $0.2-0.5$ (about 3-4h in total).

○ While cells are shaking, label $50 \times 2 \mathrm{~mL}$ microcentrifuge tubes "DH5 $\alpha$ " and store at $20^{\circ} \mathrm{C}$ in $-80^{\circ} \mathrm{C}$ storage box.

- Once the OD is between 0.2-0.5, split the culture into 2 aliquots of $\sim 25 \mathrm{~mL}$ in $50 \mathrm{~mL}$ centrifuge tubes, weighing the tube with the inoculum on a gram scale and adjusting to equal weights.

- Store tubes on ice for $10 \mathrm{~min}$.

- Centrifuge tubes for $10 \mathrm{~min}$ at $3000 \mathrm{rpm}$ at $4^{\circ} \mathrm{C}$.

- Remove supernatant by pouring carefully so as not to dislodge the pellet, and then immediately place tubes back on ice.

- Use a $1000 \mu \mathrm{L}$ pipette to remove any remaining supernatant, avoiding the pellet.

- Gently re-suspend the pellet in TSS buffer equal to $10 \%$ previous volume (i.e., use $2.5 \mathrm{~mL}$ TSS to re-suspend the cells in each tube).

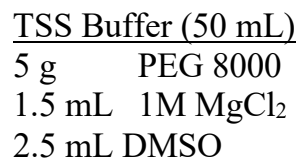

$>$ Add LB liquid media up to $50 \mathrm{~mL}$

- Very quickly, aliquot $100 \mu \mathrm{L}$ of cell suspension to each of the $50 \times 2 \mathrm{~mL}$ microcentrifuge tubes that you prepared earlier, trying to keep the box of tubes as cold as possible.

- Immediately store the box of microcentrifuge tubes at $-80^{\circ} \mathrm{C}$. 


\section{Plasmid Mini-Preps from Bacteria}

- Shake bacteria in $4 \mathrm{~mL}$ of Circlegrow ${ }^{\circledR}$ broth with appropriate antibiotic for plasmid selection overnight at $37^{\circ} \mathrm{C}$

- Spin down cells for $1 \mathrm{~min}$ at $14,000 \mathrm{rpm}$ in a $2 \mathrm{~mL}$ microcentrifuge tube $2 \mathrm{~mL}$ at a time, pouring off the supernatant after each spin.

- After the second spin, bang the tubes dry on a paper towel and add $250 \mu \mathrm{L}$ of resuspension buffer.

- Vortex the tubes to resuspend.

- Once the cells are resuspended, add $250 \mu \mathrm{L}$ Lysis Solution. Close the caps and gently invert 5 times.

- Lay tube on side at room temperature for $5 \mathrm{~min}$.

- After 5 min, add $250 \mu \mathrm{L}$ Neutralization solution and invert several times to mix.

- Centrifuge at $14,000 \mathrm{rpm}$ for $10 \mathrm{~min}$ at room temperature.

- After spin, remove supernatant to new $2 \mathrm{~mL}$ microcentrifuge tube, being careful to avoid any pellet or floating material.

- Add $750 \mu \mathrm{L}$ of isopropanol to the supernatant.

- Centrifuge again at $14,000 \mathrm{rpm}$ for $7 \mathrm{~min}$.

- Aspirate (or pour off) the supernatant, being careful to avoid the DNA pellet.

- Dry the tubes until all residual isopropanol has evaporate (15 min in a spin-vac, longer in a heating block)

- $\quad$ Resuspend the DNA pellet in $50 \mu \mathrm{L}$ TE (use filter tips)

\section{Reagents:}

Cell Resuspension Solution $<*$ We just order this premade

$50 \mathrm{mM}$ Tris- $\mathrm{HCl}(\mathrm{pH} 7.5)$

$10 \mathrm{mM}$ EDTA

$100 \mu \mathrm{g} / \mathrm{mL}$ RNase A

Lysis Solution ( $\mathrm{NaOH} / \mathrm{SDS})$

$100 \mu \mathrm{L} 10 \mathrm{~N} \mathrm{NaOH}$

$500 \mu \mathrm{L} 10 \%$ SDS

$4.4 \mathrm{~mL} \mathrm{diH} \mathrm{H}_{2} \mathrm{O}$

Neutralization Solution (1.32M Potassium Acetate)

$12.95 \mathrm{~g}$ of Potassium Acetate

$100 \mathrm{~mL}$ of $\mathrm{diH}_{2} \mathrm{O}$

$>\mathrm{pH}$ the solution to 4.8 using Glacial Acetic Acid

TE Buffer

$10 \mathrm{mM}$ Tris- $\mathrm{HCl}(\mathrm{pH} 7.5)$

1 mM EDTA 


\section{Protoplasting Microbotryum}

\section{Making Enzyme Solution}

$\begin{array}{lll}\circ & \text { Lysing Enzymes from Trichoderma harzianum } & 0.5 \mathrm{~g} \\ \circ & \text { Driselase } & 0.5 \mathrm{~g} \\ \circ & 1 \mathrm{M} \mathrm{MgSO} 4 & 25 \mathrm{~mL}\end{array}$

- $\quad$ Mix and store overnight at $4^{\circ} \mathrm{C}$

- Spin down solution at 11,000 rpm for $10 \mathrm{~min}$.

- Filter supernatant into new sterile container (50 mL Falcon Tube)

- $\quad$ Store at $4^{\circ} \mathrm{C}$

\section{Protoplasting Microbotryum (Mv) Cells}

- Grow Mv cells on YPD- $10 \%$ for 2 days at $27^{\circ} \mathrm{C}$

- Scrape a generous loop of cells into $5 \mathrm{~mL}$ of Enzyme Solution and shake on table top at mediumlow speed overnight

- CAREFULLY add $5 \mathrm{~mL}$ of $1.2 \mathrm{M}$ mannitol TOPWISE (DO NOT MIX!)

- Centrifuge at 2,000 rpm for $20 \mathrm{~min}$

- Using a P1000, carefully extract the middle layer solution without taking any of the bottom layer. (if you mix the layers you will NOT be able to re-separate them)

- $20 \mathrm{~mL}$ of solution makes about $6 \mathrm{~mL}$ of cells

\section{Short-term Storage}

- Aliquot $100 \mu \mathrm{L}$ into $600 \mu \mathrm{L}$ tubes and store in fridge until ready to use

\section{Long-term Storage}

- Aliquot $100 \mu \mathrm{L}$ into $600 \mu \mathrm{L}$ tubes and spin down cells at 14,000 rpm for $1 \mathrm{~min}$

- Discard supernatant and re-suspend cells in $100 \mu \mathrm{L}$ of STC via finger vortex

- Store in $-80^{\circ} \mathrm{C}$ until ready to use (make sure to thaw on ice before use)

*Method by Naoko Fujita 


\section{Topo Cloning}

Set up the following:

$\begin{array}{ll}\text { PCR product } & 0.5-4 \mu \mathrm{L} \\ \text { Salt Solution } & 1 \mu \mathrm{L} \\ \text { PCR-TOPO } & 1 \mu \mathrm{L} \\ \text { Total } & 6 \mu \mathrm{L}\end{array}$

- Mix gently and incubate for exactly $5 \mathrm{~min}$ at room temp

- Briefly centrifuge and place on ice. Proceed immediately to transformation

- Stir solution gently with pipette tip

- Add $4 \mu \mathrm{L}$ of the TOPO-Cloning reaction into a vial of One Shot cells and mix again

- Incubate on ice for $30 \mathrm{~min}$

- Heat shock cells for $30 \mathrm{sec}$ at $42^{\circ} \mathrm{C}$ (Gel room heat bath)

- Immediately transfer tubes to ice and incubate for $2 \mathrm{~min}$

- Cap the tube tightly and shake the tube horizontally at $37^{\circ} \mathrm{C}$ for $30 \mathrm{~min}-1$ hour

- Spread 50-100 $\mathrm{L} \mathrm{L}$ from each transformation on a plate (warm at room temp for $20 \mathrm{~min}$ ) and incubate overnight

- at $37^{\circ} \mathrm{C}$

- Pick white colonies and 1 blue for comparison

- Plate colonies on LB with $50 \mu \mathrm{L} / \mathrm{mL}$ Kanamycin or $200 \mu \mathrm{L} / \mathrm{mL}$ Ampicillin

- Miniprep

\section{Materials}

\section{$\underline{\text { LB Plates }}$}

$1.0 \%$ Tryptone

$0.5 \%$ Yeast Extract

$1.0 \% \mathrm{NaCl}$

$15 \mathrm{~g} / \mathrm{L}$ Agar

$\mathrm{pH} 7.0$

*For 1 liter, dissolve $10 \mathrm{~g}$ tryptone, $5 \mathrm{~g}$ yeast extract, $10 \mathrm{~g} \mathrm{NaCl}$ and $15 \mathrm{~g}$ agar in $950 \mathrm{~mL}$ deionized water

*Adjust the $\mathrm{pH}$ of the solution to 7.0 with $\mathrm{NaOH}$ and bring the volume up to 1 liter

*Autoclave on liquid cycle for $20 \mathrm{~min}$ at $15 \mathrm{psi}$. Allow solution to cool to $55^{\circ} \mathrm{C}$. Add antibiotic

*Pour into plates

\section{$\underline{X-G a l ~ S t o c k}$}

$40 \mathrm{mg} / \mathrm{mL}$ stock solution

*Dissolve 400mg X-Gal in 10mL dimethylformamide

*Protect from light by storing in brown bottle at $-20^{\circ} \mathrm{C}$

*Add $40 \mu \mathrm{L}$ to plates

IPTG Stock

$100 \mathrm{mM}$ stock

*Dissolve $238 \mathrm{mg}$ of IPTG in $10 \mathrm{~mL}$ deionized $\mathrm{H}_{2} \mathrm{O}$

*Filter-sterilize and store in $1 \mathrm{~mL}$ aliquots at $-20^{\circ} \mathrm{C}$

*Add $40 \mu \mathrm{L}$ to plates 


\section{Transforming Competent $E$. coli}

- Thaw competent cells on ice for 5 minutes.

- Add $5 \mu \mathrm{L}$ of plasmid and finger vortex. Quick spin.

- Incubate on ice for 30 minutes

- Quickly, move tubes to $40^{\circ} \mathrm{C}$ water bath for $30 \mathrm{sec}$, and then return to ice for $2 \mathrm{~min}$.

- $\quad$ Add $250 \mu \mathrm{L}$ of Circlegrow ${ }^{\odot}$ and shake tubes on their sides at $37^{\circ} \mathrm{C}$ for 1 hour.

- $\quad$ Spread $150 \mu \mathrm{L}$ onto selective LB media.

- $\quad$ Incubate plates at $37^{\circ} \mathrm{C}$ overnight $(\sim 18 \mathrm{~h})$. 


\section{Yeast Miniprep}

- Grow Cells in $3 \mathrm{ml}$ Dropout media to an $\mathrm{OD}_{600}>1$

-Overnight: use all $3 \mathrm{~mL}$

-Two days: use $1.5 \mathrm{~mL}$

- Pellet cells in a $1.5 \mathrm{~mL}$ microfuge using a 5 min spin. Pour off supernatant. Repeat until all of the media is

- $\quad$ pelleted. Dry on paper towel.

- Resuspend by vortexing in $200 \mu \mathrm{L}$ of SCE/Zymolyase/2ME.

- Incubate at $37^{\circ}$ for 30-60 min.

- Add $400 \mu \mathrm{L} 0.2 \mathrm{~N} \mathrm{NaOH} / 1 \%$ SDS (lysis solution). Invert to mix.

- Incubate on ice $5 \mathrm{~min}$.

- $\quad$ Add $300 \mu \mathrm{L}$ cold $3 \mathrm{M}$ K/5M OAc. Invert to mix.

- Incubate on ice $5 \mathrm{~min}$.

- Spin 2 minutes at top speed in a microcentrifuge.

- Pipette supernatant into a fresh tube. Repeat spin.

- Transfer entire volume to a fresh tube.

- Add $400 \mu \mathrm{L}$ isopropanol. Vortex and let stand for $5 \mathrm{~min}$ at room temp.

- Spin at top speed for 5 min at room temp.

- Pour off supernatant and wash pellet with $0.5 \mathrm{~mL} 70 \%$ ethanol.

- Repeat spin. Pour off supernatant.

- Dry pellet and resuspend in $25 \mu \mathrm{L}$ TE.

- Transform into E. coli. Use $1 \mu \mathrm{L}$ for electrocompetent cells or $10 \mu \mathrm{L}$ for chemically competent cells.

\section{Materials}

SCE Solution $(100 \mathrm{~mL})$

$18.2 \mathrm{~g} \quad 1 \mathrm{M}$ sorbitol (in $\mathrm{H}_{2} \mathrm{O}$ ) [ $74.8 \mathrm{~mL}$ for $\left.18.2 \mathrm{~g}\right]$

$2.94 \mathrm{~g} \quad 0.1 \mathrm{M}$ sodium citrate $\mathrm{pH} 7.6$ (dehydrate trisodium salt mwt. 294.10)

$2.23 \mathrm{~g} \quad 0.06 \mathrm{M}$ EDTA

SCE/Zymolyase/2ME Solution

$5 \mathrm{~mL} \quad \mathrm{SCE}$

$60 \mu \mathrm{L} \quad 10 \mathrm{mg} / \mathrm{ml}$ Zymolyase (in 1M Sorbitol)

$10 \mu \mathrm{L} \quad 2$-mercaptoethanol \{add in hood\}

$\mathrm{NaOH} / \mathrm{SDS}$ Solution (Lysis solution)

$100 \mu \mathrm{L} \quad 10 \mathrm{~N} \mathrm{NaOH}$

$500 \mu \mathrm{L} \quad 10 \%$ SDS

$4.4 \mathrm{~mL} \quad \mathrm{dH}_{2} \mathrm{O}$

$\underline{3 \mathrm{M} \mathrm{K} / 5 \mathrm{M} \mathrm{OAc}}$

$60 \mathrm{~mL} 5 \mathrm{M}$ potassium acetate

$11.5 \mathrm{~mL}$ glacial acetic acid

$28.5 \mathrm{ml} \quad \mathrm{dH}_{2} \mathrm{O}$ 
CURRICULUM VITAE

William C. Beckerson

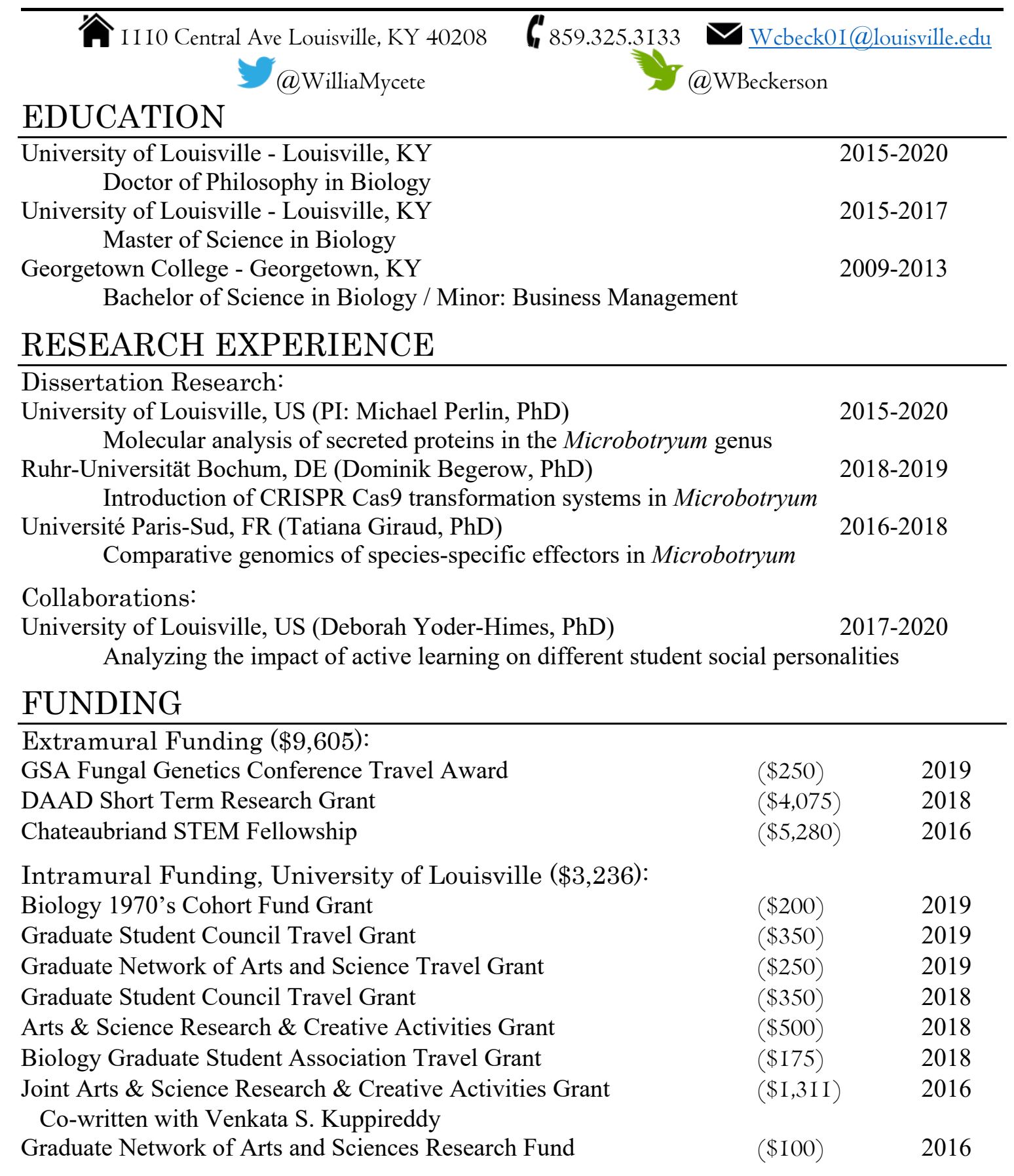


Significant Contributions to Other Grants $(\$ 296,889)$ :

NSF Track I International Research Experience for Students (IRES) ～(\$296,889) 2018

Co-written with: (PI) Dr. Michael H. Perlin Award number: 1824851

\section{PUBLICATIONS}

Publications in Progress:

Beckerson WC, Klenner S, Leiter R, Khanal S, Gold S, Dominik B, Perlin MH. (2020). The First Cut is the Deepest: Implementing CRISPR-Cas9 for site specific gene disruptions in the fungal pathogen complex Microbotryum vioalaceum. xxxxxxx. - In Progress

Beckerson WC, Klenner S, Sullivan PM, Rollnik T, Rodriguez de la Vega R, Giraud R, Begerow D, Perlin MH. (2020). To Each Their Own: Analyzing species-specific small secreted proteins in the Microbotryum violaceum species complex. xxxxxxxx. - In Progress

Beckerson WC, Long G, Rodriguez de la Vega R, Giraud R, Perlin MH. (2020). Breaker of Chains: Functional characterization of the conserved Microbotryum effector MVLG_0225. xxxxxxxx. - In Progress

Beckerson WC, Perlin MH. (2020). Director's Cut: How to design a CRISPR Cas9 construct for use in a new system. $x x x x x x x x$. - In Progress

Peer Reviewed Articles:

Beckerson WC, Anderson JO, Perpich JD, Yoder-Himes D. (2020). An Introvert's Perspective: Analyzing the impact of active learning on social personalities in an upper-level biology course. Journal of College Science Teaching. 49:3, 47-57 https://www.nsta.org/store/product_detail.aspx?id=10.2505/4/jest20_049 $03 \quad 47$

Beckerson WC, de la Vega RCR, Hartmann FE, Duhamel M, Giraud T, Perlin MH. (2019). Cause and Effectors: Whole genome comparisons reveal shared but rapidly evolving effector sets among host-specific plant-castrating fungi. mBio. mBio 10:e02391-19 https://doi.org/10.1128/mBio.02391-19

Kuppireddy VS, Uversky VN, Toh SS, Tsai M-C, Beckerson WC, Cahill CC, Carman B, Perlin MH. (2017). Identification and initial characterization of effectors of an anther smut fungus and the potential host target proteins. International Journal of Molecular Science. 18,2489 https://doi.org/10.3390/ijms18112489

Textbooks:

Perlin MH, Beckerson WC, Gopinath A, Cobbs G. (2020). Molecular and Cellular Genetics: Laboratory Studies. San Diego, CA: Cognella Academic Publishing. $2^{\text {nd }}$ Edition

Perlin MH, Beckerson WC, Gopinath A, Cobbs G. (2018). Molecular and Cellular Genetics: Laboratory Studies. San Diego, CA: Cognella Academic Publishing. $1^{\text {st }}$ Edition

\section{HONORS AND AWARDS}

Graduate Student Publication Award, UofL

Graduate School of Arts and Sciences Student Spotlight, UofL

https://louisville.edu/graduate/student-spotlight/student-spotlight-february-2020

Introductory Biology Lab Development Award, UofL

Graduate Student Research Presentation Award, UofL

Biology Department Service Award, UofL

College of Arts and Science Student Profile, UofL

https://louisville.edu/artsandsciences/academics/graduate-education/studentprofiles/beckerson 


\section{CONFERENCE PRESENTATIONS}

Oral Presentations:

Ruhr-Universität Bochum Microbotryum Symposium, DE

An Unorthodox CRISPR Approach for and Unorthodox Fungus

Asilomar Fungal Genetics Conference: Smut Convergence, US

Cause and Effectors: Secretome comparison of members from the anther-smut pathogen species complex, Microbotryum violaceum

Gordon Research Seminar on Cellular and Molecular Fungal Biology, US

The First Cut is the Deepest: Implementing CRISPR Cas9 as a transformation system for site specific gene disruptions in the fungal pathogen species complex Microbotryum violaceum

Kentucky Academy of Science Conference, US

Identifying unique small secreted proteins in divergent species of the fungal pathogen complex

Microbotryum violaceum

Ruhr-Universität Bochum Microbotryum Symposium, DE

Analyzing the role of protein-protein interactions in host/pathogen co-evolution

Poster Presentations:

National Association of Biology Teachers: Professional Development Conference, US

An Introvert's Perspective: Analyzing the impact of active learning on multiple levels of class social personalities in an upper-level biology course

Asilomar Fungal Genetics Conference, US

Cause and Effectors: Secretome comparison of members from the anther-smut pathogen species complex, Microbotryum violaceum

Gordon Research Conference on Cellular and Molecular Fungal Biology, US

The First Cut is the Deepest: Implementing CRISPR Cas9 as a transformation system for site specific gene disruptions in the fungal pathogen species complex Microbotryum violaceum

\section{PROFESSIONAL PRESENTATIONS}

Invited Talks:

Georgetown College Invited Speaker Seminar, US

Cause and Effectors: How rapidly evolving effectors lead to host-specificity between Microbotryum and Caryophyllaceae

Belknap Academic Building Anniversary Event, US

An Introvert's Perspective: Analyzing the impact of active learning on multiple levels of class social personalities in an upper-level biology course

Ruhr-Universität Bochum Invited Speaker, DE

The History and Future of CRISPR Cas9

Ruhr-Universität Bochum Invited Speaker, DE

The First Cut is the Deepest: CRISPR Cas9 and how to get started

Georgetown College Invited Speaker Seminar, US

Here and Back Again: A GCPALS tale

Chateaubriand Fellow Research Plan, FR

Identification of Small-Secreted Proteins in the Microbotryum genus

Departmental Talks:

University of Louisville Awards Day, US

An Introvert's Perspective: The effect of social personality on active learning

University of Louisvilla GRADtalks, US

Cause and Effectors: Comparing the secretomes of anther-smuts

University of Louisville GNAS Invited Speaker, US

Cause and Effectors: Secretome comparison of members from the anther-smut pathogen species complex, Microbotryum violaceum

University of Louisville Awards Day, US

Searching for "Nuclear" Arms: Identifying species-specific small secreted proteins from the fungal pathogen species complex Microbotryum violaceum

University of Louisville Awards Day, US

The First Cut is the Deepest: How to get started using CRISPR Cas9 in YOUR lab 
University of Louisville Awards Day, US

Identification of protein-protein interactions of host/pathogen co-evolution in Microbotryum violaceum

\section{TEACHING EXPERIENCE}

\begin{tabular}{|c|c|c|c|c|}
\hline \multicolumn{5}{|c|}{$\begin{array}{l}\text { Adjunct Faculty of Record, Georgetown College } \\
\text { BIO II I: Introductory Biology for Majors }\end{array}$} \\
\hline I section & $75 \mathrm{~min} /$ class & 24 students & Twice/week & Fall 2019 \\
\hline \multicolumn{5}{|c|}{ BIOL I I I: Introductory Biology Lab } \\
\hline I section & II $0 \mathrm{~min} /$ class & 24 students & Once/week & Fall 201 \\
\hline
\end{tabular}

Invited Group Lecturer for Biotechnology Methods, University of Louisville

BIOL 4I6: Biotechnology Methods (Yeast-Two-Hybrid Systems)
2 sections
$240 \mathrm{~min} /$ class
4 students
Twice/week
Fall 2018

Microbiology Teaching Innovation Learning Lab, University of Louisville

BIO 357: General Microbiology

\begin{tabular}{|c|c|c|c|c|}
\hline \multicolumn{5}{|c|}{1 section $\quad 75 \mathrm{~min} /$ class } \\
\hline \multicolumn{5}{|c|}{ BIO 357: General Microbiology } \\
\hline I section & $75 \mathrm{~min} /$ class & 49 students & Four/Semester & Fall 2018 \\
\hline \multicolumn{5}{|c|}{ BIO 357: General Microbiology } \\
\hline I section & $75 \mathrm{~min} /$ class & 43 students & Four/Semester & Spring 20I \\
\hline \multicolumn{5}{|c|}{ BIO 357: General Microbiology } \\
\hline I section & $75 \mathrm{~min} /$ class & 65 students & Four/Semester & Fall 2017 \\
\hline
\end{tabular}

Graduate Teaching Assistant, University of Louisville

BIOL 33I: Genetics and Molecular Biology

2 sections $\quad$ II $0 \mathrm{~min} /$ class

BIOL 33I: Genetics and Molecular Biology

2 sections $\quad$ II $0 \mathrm{~min} /$ class

BIOL 33I: Genetics and Molecular Biology

2 sections $\quad$ IIO $\mathrm{min} /$ class

BIOL 33I: Genetics and Molecular Biology

I section $\quad$ IIO $\mathrm{min} /$ class

BIOL I04: introduction to Biological Systems

2 sections $\quad$ IIO $\mathrm{min} /$ class

BIOL 33I: Genetics and Molecular Biology

2 sections $\quad$ IIO $\mathrm{min} /$ class

BIOL 33I: Genetics and Molecular Biology

I section $\quad$ II $0 \mathrm{~min} /$ class

BIOL 258: Microbiology

2 sections $\quad 90 \mathrm{~min} /$ class

BIOL 33I: Genetics and Molecular Biology

2 sections $\quad$ IIO min/class

BIOL 33I: Genetics and Molecular Biology

I section $\quad$ IIO $\mathrm{min} /$ class

BIOL 244: Principles of Biology

2 sections II $\mathrm{min} /$ class

BIOL I04: introduction to Biological Systems

3 sections

II $10 \mathrm{~min} / \mathrm{class}$
$20 \& 20$ students Twice/week Spring 2020

$20 \& 22$ students Twice/week Fall 2019

$20 \& 22$ students Twice/week Spring 2019

I9 students $\quad$ Twice/week $\quad$ Fall 2018

I4 students Three/week Summer 2018

$20 \& 2$ I students Twice/week Spring 2018

8 students $\quad$ Twice/week $\quad$ Fall 2017

I5 \& 6 students Four Days/week Summer 2017

I7 \& 2 I students Twice/week $\quad$ Spring 2017

I6 students Twice/week Fall 2016

$27 \& 28$ students Twice/week Spring 2016

33, 33, \& 33 students Once/week Fall 2015 


\section{STUDENTS MENTORED}

\begin{tabular}{|c|c|c|}
\hline Ms. Rebecca Turney & Perlin Lab, University of Louisville & 2020 \\
\hline Mr. Lucas Engelhardt & Begerow Lab, Universität Bochum & 2019 \\
\hline Mr. Phillip Sullivan & Perlin Lab, University of Louisville & 2018-2019 \\
\hline Ms. Grace Long & Perlin Lab, University of Louisville & 2018-2019 \\
\hline Mr. Lloyd Bartley & Perlin Lab, University of Louisville & 2017-2018 \\
\hline Mr. Adney Rakotoniaina & Perlin Lab, University of Louisville & 2017 \\
\hline Ms. Brittany Carman & Perlin Lab, University of Louisville & 2016 \\
\hline Ms. Catarina Cahill & Perlin Lab, University of Louisville & 2015 \\
\hline \multicolumn{3}{|c|}{ UNIVERSITY SERVICES } \\
\hline \multirow{3}{*}{\multicolumn{2}{|c|}{$\begin{array}{l}\text { Biology Undergraduate Student Association: Graduate Student Panel } \\
\text { Biology Faculty Search Committee - Graduate Student Representative } \\
\text { German Club }\end{array}$}} & 2019 \\
\hline & & 2019 \\
\hline & & 2018-2019 \\
\hline Student Grievance \& Discipline Committee & $\begin{array}{ll}\text { 2018-2019 Member } & \\
\end{array}$ & 2016-2017 \\
\hline 2016-2017 & Science Division Representative & \\
\hline \multicolumn{2}{|c|}{ Graduate Network of Arts \& Sciences } & 2016-2018 \\
\hline $2017-2018 \quad \mathrm{~V}$ & sident & \\
\hline 2017 & Science Rep. for Grant Review Committee & \\
\hline $2016-2018 \quad$ D & ent of Biology Representative & \\
\hline \multicolumn{2}{|c|}{ Biology Graduate Student Association } & 2015-2020 \\
\hline $2019-2020 \quad P$ & & \\
\hline 2018-2019 & Student Rep. & \\
\hline 2016-2017 & & \\
\hline $2016 \& 2020$ & & \\
\hline $2016-2020 \quad \mathrm{~N}$ & & \\
\hline
\end{tabular}

\section{COMMUNITY INVOLVEMENT / OUTREACH}

Beer with a Scientist - Monnik Beer Company, Louisville US

Our Friends the Fungi: The many types of fungi and the history of how we've used them

Orlando Science Center: Spooky Science Week

Zombie Hunt: Using iNatrualist to find zombie ants

Citizen Science Initiative: the Zombie Fungus Foray

Website: https://wcbeck0I.wixsite.com/thezombiefungusforay

iNaturalist: https://www.inaturalist.org/projects/the-zombie-fungus-foray

Skype a Scientist

2019-2020

2020 Creekside Middle School: Sixth Grade Class - Bentonville, AR

2019 Marie Curie Institute: Fourth and Fifth Grade Class - Amsterdam, NY

Corry Area High School: Ninth Grade Class - Corry, PA

Newark Central: Second Grade Class - Newark, NY

E.K. Powe Elementary School: First Grade Class (AKA the Sea Crew) - Durham, NC

Annunciation Catholic School: Seventh Grade Class - Denver, CO

Guest Speaker at University of Louisville: Meet the Professor

2019

Science Information Literacy \& Oral Communication

DuPont Manual Regional Science Fair Judge, Louisville KY

2018

2018

Louisville Regional Science \& Engineering Fair Judge, KY

2018

The history of genetic modification of our food

Guest Speaker at Lexington Christian Academy High School 2016

What is a GMO?

ExBEERiment - Socialize with Science at the Louisville Science Center 


\section{PROFESSIONAL DEVELOPMENT / SERVICES}

Training/Workshops:

University of Central Florida NIH One Day Virtual Conference

2020

Moving classes to a remote option for COVID-19

2020

Training with Panopto, Blackboard Collaborate, and Remote Assessment Tools

Research Academy RUHR: Open Access Science Workshop, DE

2019

Faculty Search Committee: Diversity Training, US

2019

Professional Societies/Organizations:

National Association of Biology Teachers

2019-2020 Community Science Committee

2019-current

Genetics Society of America

2018-current

Kentucky Academy of Science

2014-current

Peer Review

Society for Molecular Plant-Microbe Interactions

2020

\section{DIVERSITY STATEMENT}

As a first-generation college graduate, I am deeply committed to providing opportunities for individuals of all socioeconomic, educational, religious, gender, age, sexuality, nationality, disability, and racial backgrounds. Science benefits from the flow of different ideas and life experiences, and I take steps to be consciously inclusive towards all groups. I also conduct myself under the fundamental premise that quality education should be available to every individual. This includes prioritizing publication of research and data strictly in open access journals and platforms, and actively reaching out to, and providing financial support for, individuals underrepresented in STEM. Financial compensation in addition to the lab experience gained by high-school and undergraduate students is essential to alleviate barriers of entry that disproportionally affect minoritized groups and is vital to improve retention of these students. Financial support includes compensation for the student's work, as well as funds to attend conferences and present their research. As a graduate student, I mentored a diverse group of undergraduate students from a wide range of backgrounds, 6/8 of whom are from traditionally underrepresented groups in Biology. I am committed to continuing this practice of inclusivity and have established a Community Science project oriented towards increasing the participation of Latin(x) individuals, both in academia and in the local community, for future research endeavors.

\section{RESEARCH TRAVEL}

Ruhr-Universität Bochum, DE

2019 May-Aug

Graduate student leader for the IRES grant: Infection assays and electron microscopy of transgenic Microbotryum

Ruhr-Universität Bochum, DE

2018 June-July

Collaborative research project transforming Microbotryum with CRISPR Cas9.

Université Paris-Sud, FR

2016 May-Aug

Collaborative research learning horticulture and infection techniques and implementing agrobacterium-mediated transformation methods for Microbotryum.

RWTH University Aachen, DE

2016 June

Group outing with collaborators to discuss future research directions within the Microbotryum community.

Ruhr-Universität Bochum, DE

2016 June

Microbotryum symposium with collaborators from Germany and France.

USDA Georgia, US

$2015 \mathrm{Dec}$

Learning agrobacterium-mediated transformation techniques in fungi to apply to Microbotryum with Dr. Scott Gold 


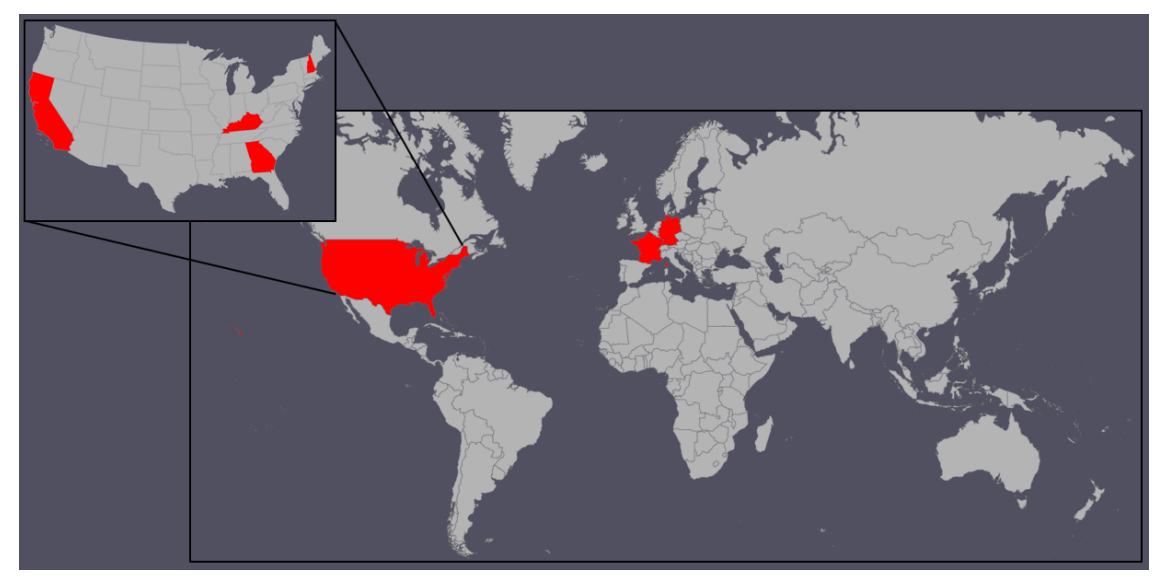

\title{
LANGUAGE PROFICIENCIES
}

\author{
English \\ IRL level 5 - Native Proficiency \\ German \\ IRL level 2 - Limited Working Proficiency \\ French \\ IRL level 2 - Limited Working Proficiency \\ Spanish \\ IRL level I - Elementary Proficiency
}

\section{REFERENCES}

Dr. Michael Perlin University of Louisville, US/ PI

Michael.perlin@louisville.edu

Dr. Tatiana Giraud

Université Paris-Sud, FR/ Collaborator

Tatiana.giraud@u-psud.fr

Dr. Dominik Begerow Ruhr-Universität Bochum, DE/Collaborator Dominik.begrow@rub.de

Dr. Scott Gold

USDA Georgia, US/ Committee Member Scott.gold@ARS.USDA.Gov 